

\section{Katurwil|jen|chaftliç̧e Bibliothef}

aus ber Sammlung Göjळen.

Э̌edes Bändchen elegant in Leintwand gebutben 80 Bfentig.

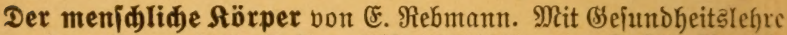
von Dr. med. $\mathfrak{H}$. Seiler. Mit 47 शłbbild. u. 1 Tajel. शr. 18.

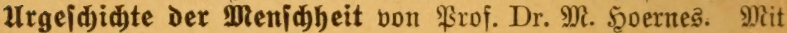

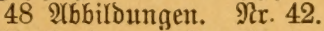

Bölferfunde von Dr. $\mathfrak{M}$. Shaberlandt. Mit 56 2rbbild. $\mathfrak{9 x} 73$.

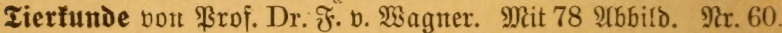

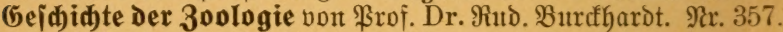
Tierbiologie von ßrof. Dr. S.. Simroth. Nr. 131.

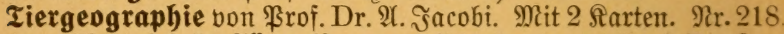
Das Tierreid I: Säugetiere bon §berftndienrat \$rof. Dr. Rarl Sampert. Mit 15 2(bbiloungen. Nir. 282.

- III: Reptilien und $\mathfrak{A m p h i b i e n ~ v o n ~ D r . ~ F r a n z ~} \mathfrak{S e r n e r , ~}$ Pribatoozent an D. Univ. WSien. Mit 48 2rbbild. Nr. 383.

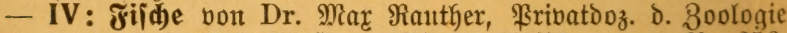

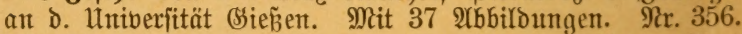

- VI: Die wirbellojen Tiere von Dr. \&nowig Böhmig, \$ro feffor Der 8oologie an Der Uniberjität Braz. I: Irtiere,

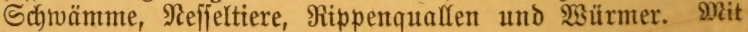
74 Figuren. Nir. 439.

Entwidlungageidjidite Der Tiere von Dr. Johs. Meifenheimer, łrofeffox ber Bonfngie an Der Univerittät Mrarburg. I: Fur=

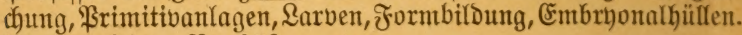
Mit 48 Fig. $\mathfrak{N r}$. 378.

- II: Drganbilloung. Mit 46 Figuren. Nr. 379.

Sd)marober und Sdmarogertum in Der Tierwelt bon \$rof. Dr. F. v. Wagner. Mit 67 NCbbildoungen. Na. 151.

Die Pflanze von \$rofefior Dr. E. Dennert. Mit 96 श्tbbiloungen. $\mathfrak{N i x} 44$.

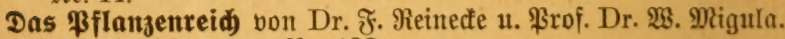
Mrit 50 F̌tgurent. $\Re$ r. 122.

Pflanzengeographie von \$rof. Dr. Rubrig Diels. Nir. 389.

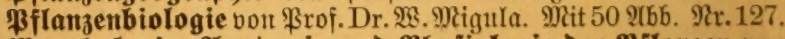

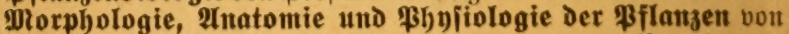

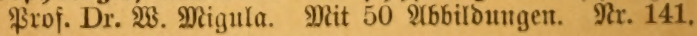


Die ßflanzenwelt Der Gewäffer von ßrof. Dr. W. Migula. Mit 50 2(bbildungen. $\mathfrak{N r} .158$.

Cxfurionsflora von Dentidland zum Beptimmen der bäu= figeren in Dentidland wilDwađjenden Pflanzen. 2 Bänd= then. Mit 100 24b bildungen. Sir. 268, 269.

Die Nadelfölzer vou ßrof. Dr. F. $\mathfrak{S}$. Reger in Ifarandt. Mit 85 Abbildoungen, 5 Iabellen und 3 Sarten. Na. 355.

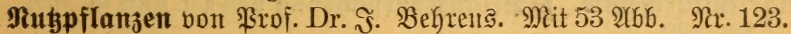

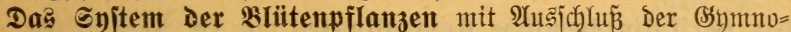

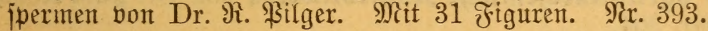
Die Pflanzenfranflyeiten bon Dr. Werner Friedrich Butde in

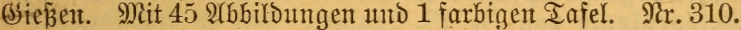

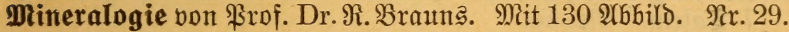

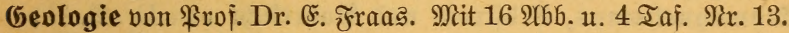

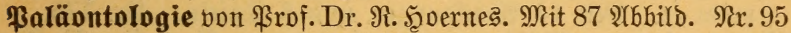
Petrographie von \$rof. Dr. $\mathfrak{B}$. Bruhns. Mit vielen $\mathfrak{A b G i l}$ Dutugett. Nr. 173.

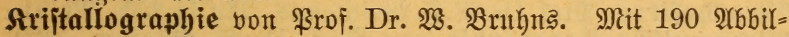
duttgent. Nir. 210.

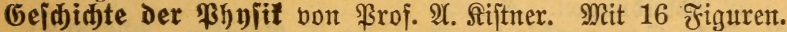
2 Bäntoe. গ̂lx. 293, 294.

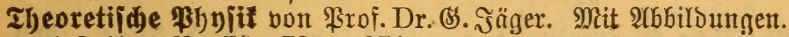
4 Teile. Nr. $76-78$ it. 374.

Radioaltivität von Wilf. Frommel. Mit 18 Figuren. $\mathfrak{N}$. 317. \$byjifalijøe Mtefiungsmethoden von Sberlebrer Dr. Wilf. Bafrot. Mit 49 Figuren. Nir. 301.

(5ejdidte Der Chemie von Dr. Feltgo Bauter. I: ßon den äls

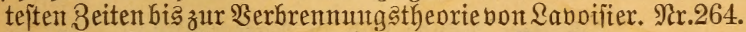

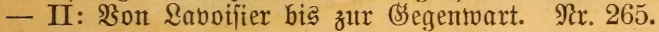

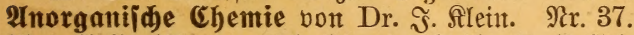

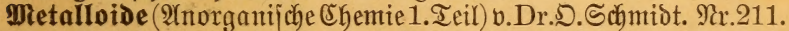

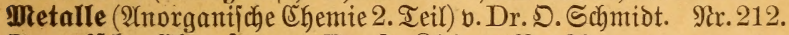

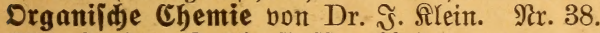

Chemie Der Soblentoffuerbindungen bon Dr. 5. Bauer. 4. Teile. $\mathfrak{N x}$ 191-194.

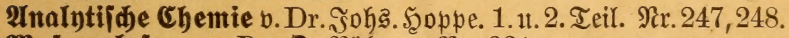

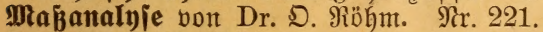

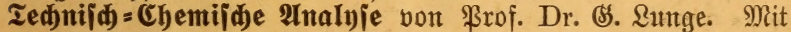
16 श्रbbiloungen. গNi. 195.

Stereodemie von \$rof. Dr. ⿷匚. Wedefind. Mit 34 Fig. NR. 201. 


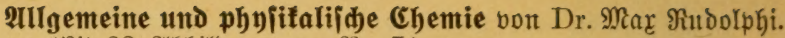
Mit 22 abbiloungen. Nir. 71 .

Eleftrodemie von Dr. Şeinr. Danneel. I: Theoretijhe Elef:= trochemie und ifre phyjiffalijch=chemijchen Srnmblagen. Mit 18 Æiguren. গ̊r. 252.

- II: Experimenterle Ẽteftrochemie, Meß̃metgoden, Reitfähigfeit, Qöjungen. Mit 26 Figuren. Nix. 253.

श्Fgrifulturđemie. I: Pflanzenernäbrung von Dr. Sarl (Srauter. Nix. 329.

Das agrifulturdemijðe Sontrollweien bon Dr. \$aul Srijøe. গix. 304.

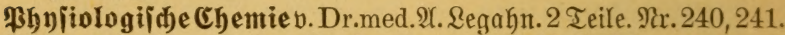
Meteorologie von Dr. W. Trabert. Mit 49 24bbildungen und 7 Tafelit. Nr. 54.

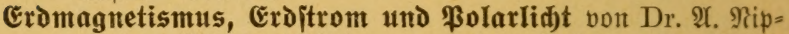

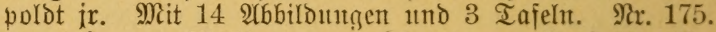

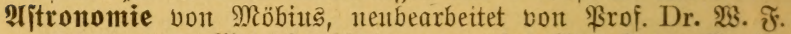

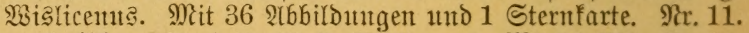

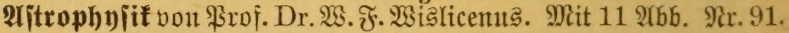

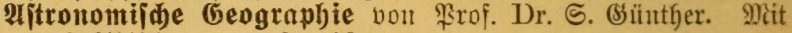
52 2rbbildunget. ?ir. 92.

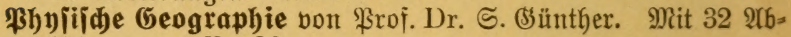
biloutngen. গir. 26.

Bhyitide Meèrestunde von Prof. Dr. Berhard Schott. Mit 28 श्bbildungen und 8 Tafeln. Nir. 112.

Slimafunde. I: Allgemeine Slimalel)re von \$rof. Dr. 23.

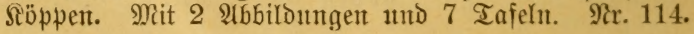

Weitere $\mathfrak{B a ̈ n d e ~ f i n t o ~ i n ~} \mathfrak{B}$ orbercituntg. 
Anardehuary. 

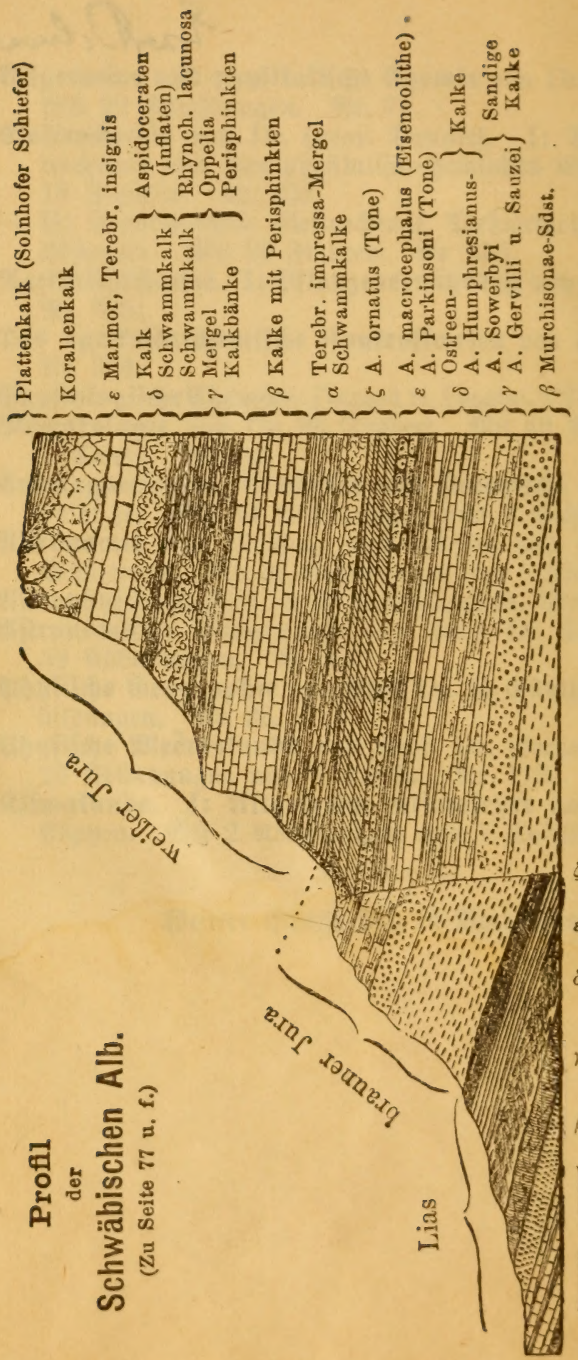

ช

$\zeta$ Jurensis- $\}$ Mergel

PosidonomyaSchiefer

$\delta$ Amaltheen-Tone

Terebrat. numismalizMergel

$\left.\begin{array}{l}\begin{array}{l}\text { Rarikostaten- } \\ \text { Oxynoten- } \\ \text { Tuneri- }\end{array}\end{array}\right\}$ Tone

Ob. Arieten-Bk.

$\alpha$ Unt. Arieten-Bk.

Angulaten-Bk.

Psilonoten-Bänke.

Keuper 


\section{Sammtluntg Bspächent}

\section{Geologie}

in furzem $\mathfrak{A} \mathfrak{n t s} z \mathfrak{g}$

für Schulen und zur Selbitbelehrutg zujammengeftellt non

\section{Prof. Dr. Eberbard Jraas}

Mit 16 2hbbildungent unt 4 Iajeln mit 51 Figuren

Dritte, verbefferte $\mathfrak{A}$ uflage

Fünfter 2 Gborud

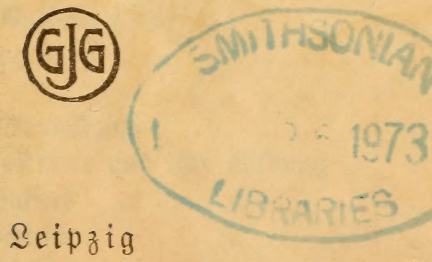

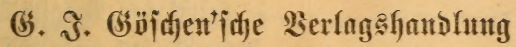


भrre redite, insbefondere das überfetungsredt, von ber Berlagshandung vorbehalten.

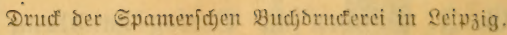




\section{Jnhaltaverzeichnis.}

Xufgaben der Beologie . . . . . . . . . 7 Material ber Erofruite.

Eintetlung . . . . . . . . . 10

Einfache Befteine. . . . . . . . . . 11

Siemengte SEefteine . . . . . . . . . 18

1. Eruptio = oder maffige Sefteine . . . 18

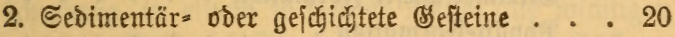

Trummer=હ゙efteine . . . . . . . . . . 21

Cntitehung Der Befteine.

Bulfanifge Birbungen . . . . . . . 22

Dex જ̌ju . . . . . . . . . . . . 22

X̂fuf́au einesి Bulfanes . . . . . . . . 25

Stratobulfane . . . . . . . . . 26

Maffige Bulfane . . . . . . . . . . 27

Maare . . . . . . . . . . . . 28

Eritarrungserjcheinunget. . . . . . . . . 28

Einteilung ber \$ulfangefteine . . . . . . . 30

Begleiterideinungen . . . . . . . . . 30

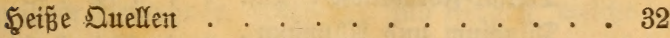

Theorie der $\mathfrak{B u l f a n t b i l d u n g ~ . ~ . ~ . ~ . ~ . ~ . ~ . ~} 32$

Die Sedimentärgefteine und thre Birbung . . . 33

Tätigfeit des ærafiers . . . . . . . . . 34

Sriftalliniface Schiejer. . . . . . . . . 34

Tätigleit des cifes . . . . . . . . . 35

Tătigfeit des Wintes. . . . . . . 36

Tätigleit ber Drganisెmen . . . . . . . 37 
Bildung Der (Erobberiläd) .

Rontraftion ber Frorinde . . . . . . 40

Bsebingstilloung . . . . . . . . . . . 42

Erobeben . . . . . . . . . . . . 45

Dả $\mathfrak{W}$ affer und feine Arbeit . . . . . . 46

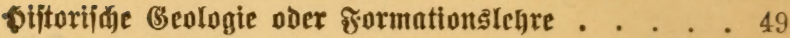

Fazieguerjøiedentheit . . . . . . . . . 50

Begriff ber Formation . . . . . . . . 51

AYterşeftimmung ber ₹rormation . . . . . . 51

Sliederung ber formation . . . . . . . . 52

Erftes Beitalter. . . . . . . . . . . 53

Sineis= Formation . . . . . . . . . 54

SYlinmerjhiefer=formation . . . . . . . 54

Phyllit=Formation . . . . . . . . . 55

8 tweites Beitalter . . . . . . . . . 56

গambrium und Silur=Formation . . . . . 58

Debon $=$ Formation . . . . . . . . 61

Rarbon=formation . . . . . . . . . . . 64

Permijche oder Dyas= Frormation . . . . . . 68

Drittes Beitalter. . . . . . . . . . . 69

Irias= Formation . . . . . . . . . . 71

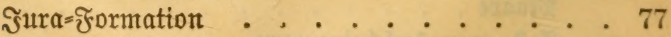

Ireide=frormation . . . . . . . . . 83

Biertez Beitalter . . . . . . . . . 87

Tertiär=formation . . . . . . . . . . 88

Dilubium und 201 utuium . . . . . . . . . 91

8ufammenftellung ber wichtigften Pflanzen= und Tier-

verfteintungen . . . . . . . . . . . . . 94

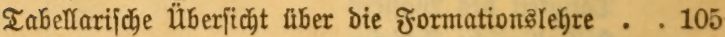

artphabetijches Regifter. . . . . . . . . . 115 


\section{Die Aufgaben der Geologie.}

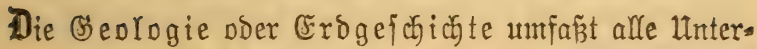

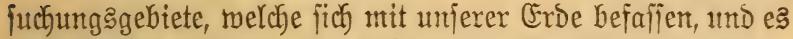

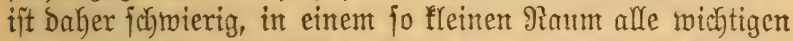

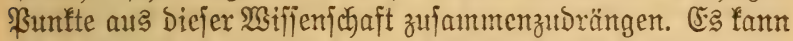

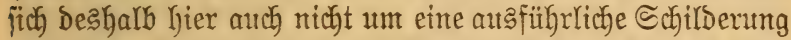

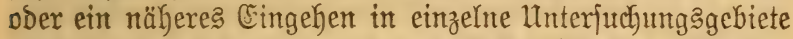

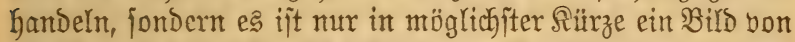

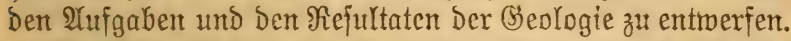

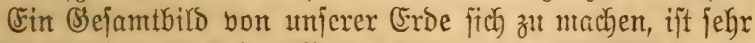

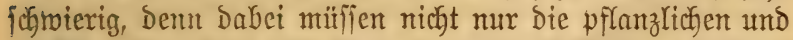

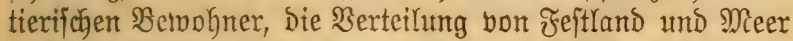

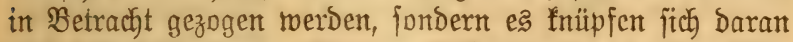

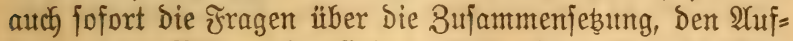

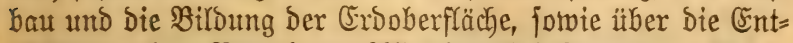

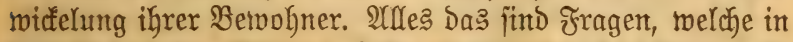
Das Brebiet ber Breologie fallen, und auf twelche fie and in

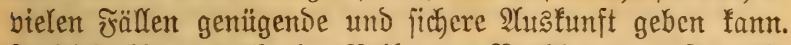

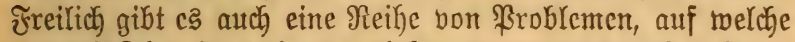
nur mit Theorien und Sygpothcicn gcantmortet werben fann;

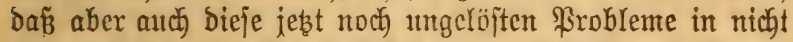
mefhr allzu langer Beit einte befriesigenbe Ertflärung finden, ift zu ertwarten, bent utrjere $\mathfrak{B}$ iffifenjfaft ift eine jegr jugend= 


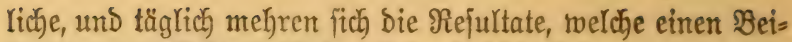
trag zu bem Sefantbild Der (Frogejofichte liefern.

Ilu ber $\mathfrak{A} u f g$ abe eines furzen úberblictes gerecht zu

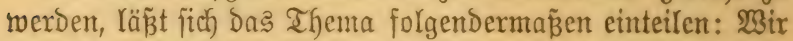

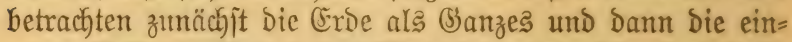
zelnen Beftandteile, D. Ђ. Dą Material, aus twelcher fite bejtebt.

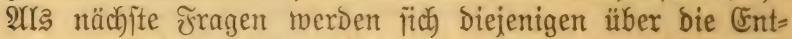
ftefungsıeije diejes Materiales und dießermendung desfelben bei Der Birloung Dex heutigen Erobberfläche ergeben. Bum Sc)luffe mun fich fobann ber eigentlich gej(hichtliche Teil an=

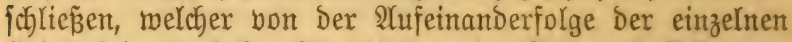
Eroperivden und Dex Entmidfelung Dex Tiex = und \$flanzen= welt handelt.

(5) wird nach Der bon fant und Saplace aufgeitellten

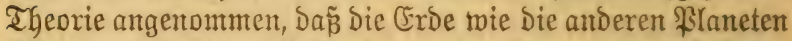
ficc) in gasförmigen Buitand bon ber Sonne abgelöft habe,

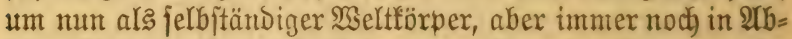
bängigfeit von ihren Entitehungspunft - ber હonne - im Weltraum zu fdumeben. Dicje Ilnuabnte ïber Den ltriprung Der (Frde ijt zmar nur eine Shupotheje, aber Dicje geminnt an

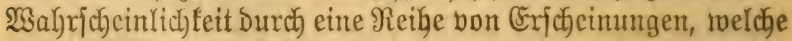
mit ify in bolfem cinflang fteben und bie fich auf andere Weije faum erflären laj̄en (fireislauf Der Ẽrbe un die Sonne,

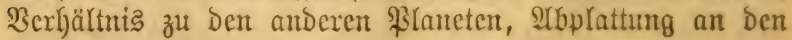
ßolen).

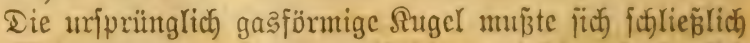
im $\mathfrak{W e l t r a u m}$ bon auß̄en lyex abtïhlen, uns bie unfprïnglich gaรförmigen (EYemente begannen fick zu verbinton und in glut=

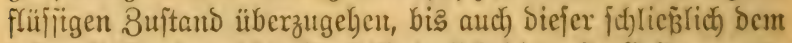

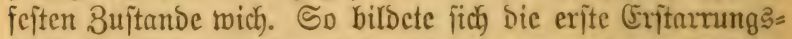
frujte Der (Eroe, melde mit Der Beit inmer melyr an Didfig= feit zunahut. Über Dic Iicte Der itarren Eroftujte fönnen wir zrwar nichts Bejtinuntes angeben, aber wir fömen anberfeitß 


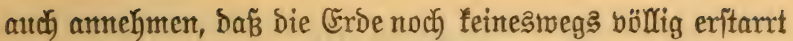

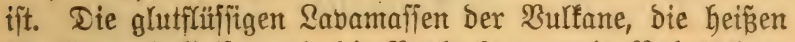
פuellen und (Seifer, wie die Beobachtungen in Bohrlöchern und Bergmerfen, relche eine j̈tetige Zunahme Der Temperatur nach Der Tiefe ergeben, betweijen uns mit Sicherbeit, Dafi im (sxbinnern noch Berbältnifje hemichen, unter Denen die Be $=$ fteine f̈ch in flïfïigem, vielleicht fogar in gasfförmig überbißztem Bujtande befinden.

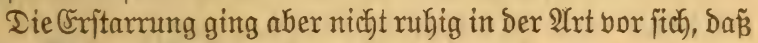
jedes (Erement für j̈th erftart ift, fondern fie rar verbunden mit grop̈artigen und tomplizierten doentijchen Borgängen, als Deren Snorejultat uns jebst Die Mineralförper entgegentreten. Tie Interfuctung Der Mineralien und ifrer (sigenjobaften ijt 2ufgabe Der Mineralogie und muñ biex als befant voraus= gejebt merden. *) Unjere 2 ufgabe ift es Dagegen, Das gejes: mäpige 8 ujantmentreten Der Mineralbeitandteile zu Beiteinen fenten zu Yernen, um einen überblid jiber Das \$્Naterial zu bes

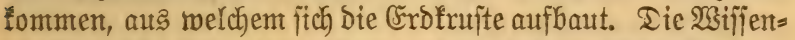

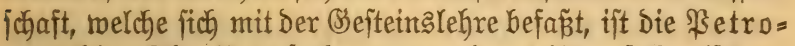
graphie. Jhre Interfuchungen toerben teils auf djemijichem, teils auf friftallographijobem $\mathfrak{W e g e}$ Durchgefithrt, und zur $\mathfrak{A} u \mathfrak{f}=$ röjung Der Sefteine in ifre Mineralbeftandteile dient bor allem Das Mifroffop. **)

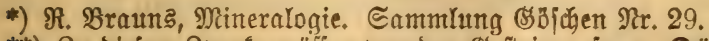

**) 8u Diejem Brecfe mülfen von den SSefteinen jogen. Dünn=

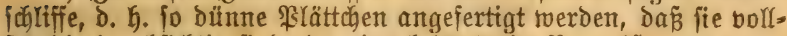

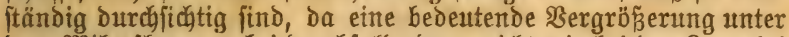
Dem Mifroffop mur bei burchfallendem, nicht trie bei Der \&upe bei auffallendem Lidgte möglich ift. 


\section{ISbichnitt. \\ Das Material der Erdkruite.}

\section{((Bciteinglchre.)}

Man unteridetibet unter Don Bemengteilen Der Beiteine mefentliche Bejtandteile, D. h. foldhe, welche für ben be= treffendon Bejteinscharafter maßjgebend find utmo in Demiclben

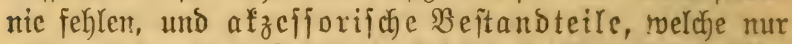
geYegentfich utnd auf @ofalitäten bejchränft in Dent betreffenden Biejtein vorkommen. Dieje afzefforifoch Bejtandteile treten

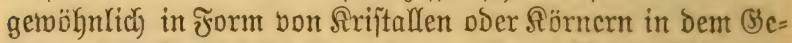

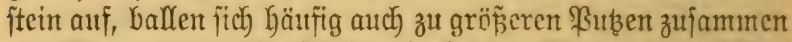
und fömen unter Uniftänden in folcher Menge ausgebildet \{ein, Daß̃ fie bie wefentfichen $\mathfrak{B e j t a n t e i l e ~ b e i ~ w e i t e m ~ u ̈ b e r = ~}$ wiegen uno jo Dem (S) ejteinstypus einen ganz berichiedenartigen

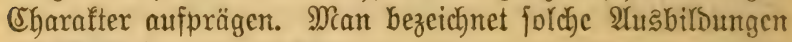
als (3) citcinsbarietäten.

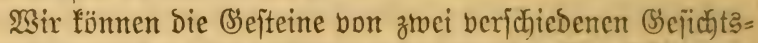
punften aus betractenten, indem wir entreder ifre Bilfumgs= meife (ß̊. wir fie auf if)re Bujammenfeksung (Betrographic) prüfer. İm exiteren Falle erlyalten wir 2 Szauptgruppen:

1. Sejteine bon majïger Struttur, bie als feuerflitfïge Mafien aus Dent Eroinnert emporgebrungen find, fog. Erup= tibgefteine.

2. Michr oder mintoer beutfich) gef(r)ictetete (Beiteine, bie ihre Bilbung auf twäfferigem $\mathfrak{S}_{\mathrm{ege}}$ Dutrch Mcercsablage= rungen ujw. Durchgemadft haben - jogen. Sebimentär = gefteine.

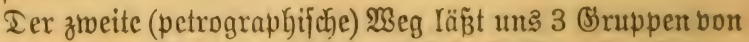

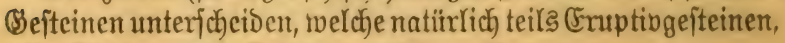
teilb Scbimtentärgeiteinen angełören fönnen: 
1. Finfache (S) fitcine, Die mur aus einer cinzigen $\mathfrak{M i}^{-}$ nerarjubitanz bejtehen.

2. Bemengte Bsefteine, Dic ats einem (Bemenge meh)= rerex Mineraljubjtanzen zujammengejest find.

3. Trümmergefteine (flajtifbe (S̈efteine), melche zum gropen Teile aut Iojen ober vertitteten Iritmmern und aus erbigen ober fandigen überreiten anderer Siejteine gebilbet finto.

\section{Einfache Gerteine.}

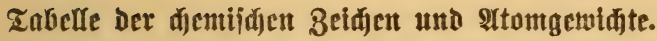

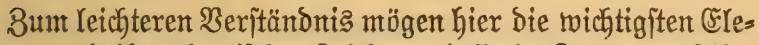
mente und ifre dyemijchen Beichen, wie fie im Texte angefübrt find, zujammengeftellt jein:

ఉеm. Beiđ. 2イtomgew.

Maliexftoff $=\mathrm{H}$

2̂luminium $=\mathrm{Al}$

$\mathfrak{2}$ ntimton $=\mathrm{Sb}$

2) rijen

Barium

Brei

$\mathfrak{B o r}$

Brom

Ehlor

Chrom

Eifen

f̧hor

Giold

รOס
$=\mathrm{As}$

$=\mathrm{Ba}$

$=\mathrm{Pb}$

$=\mathrm{B}$

$=\mathrm{Br}$

$=\mathrm{Cl}$

$=\mathrm{Cr}$

$=\mathrm{Fe}$

$=\mathrm{Fl}$

$=\mathrm{Au}$

$=\mathrm{J}$ \begin{tabular}{c|l}
1 & Rarium \\
27,4 & Rutzium
\end{tabular}

122

75

137

207

11

80

35,5

52,2

56

19

197

127

Fobalt

Sitpfer

Mangan

Natrium

Nitifel

Bratin

Platin

Scfjucfer
Sohlenitofi $=\mathrm{C}$

Magnefutum

ßryosphor

Duedfitber

Sauteritofi

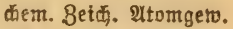

$$
\begin{array}{ll}
=\mathrm{K} & 39 \\
=\mathrm{Ca} & 40 \\
=\mathrm{Co} & 59 \\
=\mathrm{C} & 12 \\
=\mathrm{Cu} & 63,4 \\
=\mathrm{Mg} & 24 \\
=\mathrm{Mn} & 55 \\
=\mathrm{Na} & 23 \\
=\mathrm{Ni} & 58 \\
=\mathrm{P} & 31 \\
=\mathrm{Pt} & 198 \\
=\mathrm{Hg} & 200 \\
=\mathrm{O} & 16 \\
=\mathrm{S} & 32
\end{array}
$$


ఉem. Beich. Utomgem.

\begin{tabular}{llr|llr} 
Sifber & $=\mathrm{Ag}$ & 108 & Wismut & $=\mathrm{Bi}$ & 210 \\
Silizium & $=\mathrm{Si}$ & 28 & Wolfram & $=\mathrm{W}$ & 184 \\
Stidfitoff & $=\mathrm{N}$ & 14 & Binf & $=\mathrm{Zn}$ & 65 \\
Strontium & $=\mathrm{Sr}$ & 88 & Binn & $=\mathrm{Sn}$ & 118 \\
Titan & $=\mathrm{Ti}$ & 50 & Birfonium & $=\mathrm{Zr}$ & 89,6
\end{tabular}

Tie in ber Patur als Mineralien borfommenden Berbin= bungen biejer şlemente Gier aufzuzählen, liegt außerbalb des Rahmens unjerer Breologie; fie find in bem XX. Bande ber

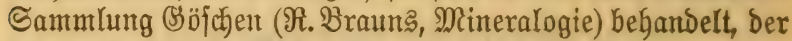

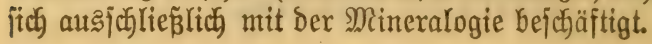

\section{Cisgejteite.}

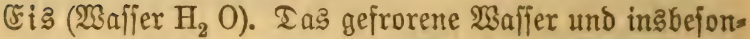
bere bas aus Schnee entĩtandene Biretfchereis bildet fomohl wegen feines majfenthaften 2 huftretens, al’ auch megen feiner untwälzenden frraft einen ïberaus wicftigen geologifidjen

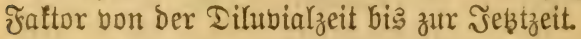

\section{Siejelgejteine.}

Duarz (Riefelfäure Si $\mathrm{O}_{2}$ ) ift an fitch) eines̉ ber Gäufigiten Mineralien, welches rejentlicke Bejtanteile ber meiften triftallinifdyen Scyiefer, Der (3ranite, Duarzporphyre, Duarz= biorite, fobann ber Sandjteine und ber meijten Tomfdyiefer

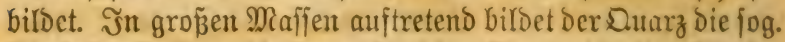
Riejelgeiteine; Gierher gehört ber Duarzit, eine förnige

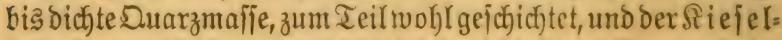
id) iefer, meiftidfwarzes, bichtes, bümichicfriges Duarzgejtein, beibe in ben ălteren હechiefern borberrfahend. Feueritein,

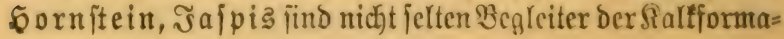


tionen; Dazu treten noch) aus Der (Simppe Dex Dpare ober Fiejel= jäure $=5$ borate in Den jüngeren Formationen die 2 bjäke heiper Duellen, jog. Riejeljinter, und die Durit Diatomeen ges

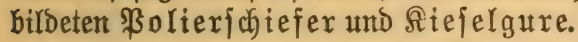

\section{Cijenterze.}

Die Eifenberbindungen treten in allen Siejteinen in grop̈er Menge auf und berleifen Denfelben bet Dex Bermitteming meift bie roftig braune Färbung. Nanthmal bilben bie Eijen= exze mächtige Ublagemungen und find beshalb als einfacte (Siefteine aufzufübren. Şierher gehört Der Brauneifenftein

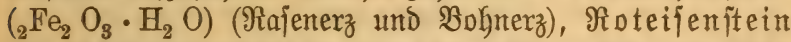
$\left(\mathrm{Fe}_{2} \mathrm{O}_{3}\right)$, bejonbers häufig als mächtige (sijenoolitbe entwidfelt, Scagneteifenftein $\left(\mathrm{Fe}_{3} \mathrm{O}_{4}\right)$, teils in gropen Lagen in ben archäijchen Formationen, teils als alzefforijocter Bejtandteil faft aller mafifigen Sefteine und Der friftallinifiben Sajiefer. Spateijenitein und Toneijenjtein, die fohlenjaure Ber=

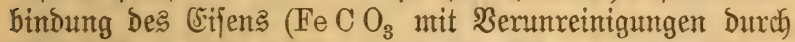
$\mathrm{Ca}, \mathrm{Mg}, \mathrm{Mn}$ und Ion), findet fich bald in (S̈ängen auşgejofieden, bald felbftändige Stödfe billdend.

In ähnlicher Weije ober mit Den Sifenerzen berbunden treten bie Mangan= mo Titanerze auf.

\section{Salze (Chlorid = unt Mitratgeiteine).}

Stein $\left\{a I_{z}(\mathrm{NaCl})\right.$. Das Eylornatrium findet fich in allen feoimentären Jormationen, too es fich Durch Berdunitung Des

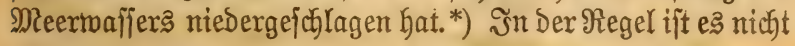

*) 1 Silo MReermaffer entfält:

Ehlornatrium . . . 27,18 Eglorfalium • 0,61 EGlormagnefum . . 3,35 Brommagnefium 0,05 jogrefeljaure Magnefia 2,27 Doppelfohtenj. Salf 0.04

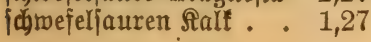

34,77 Eramm. 
oolfitändig rein, fondern berumreinigt Durd) Ton unD $\mathfrak{T}$ nhbybrit

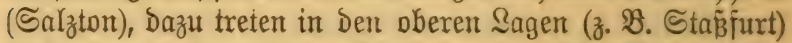

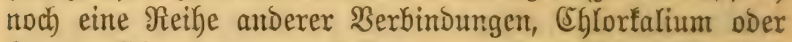
Snlvin, Ehlorfalżium und Ehlormagnejüm (Carnallit). Die Mëchtigfeit ber ऽalzlager ift oft eine ganz enorme; bei Speren=

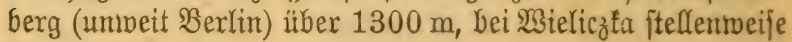
über $\left.1400 \mathrm{~m} .{ }^{*}\right)$

Bon groper tedynifder Wictigfeit find bie Salpeter=

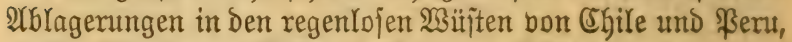
welche Sitrate bon Sali und Niatrou entfalten.

\section{Sarbonate.}

Tas Gäufigfte Sarbonat ift ber tohlenfaure Salt $\left(\mathrm{Ca} \mathrm{CO}_{3}\right)$. Salffpat und $\Re$ altitein, weldyer Den größ̈ten Teil ber Sebintentärformationen birbet. Se nach) Der Strutur und Benunreinigung Durch Ton unterfdyeibet man cine Reibe von Barietäten. Marmor ober förniger Ralf tritt meift in Den alten Formationen auf; gemeiner ßalfitein ijt bon fehr feinem Sorn und gemöhnlich Durch frembe Mineraljubjtanzen (Ton, Siejelerde, Dolomit, (Fifen unt Bitumen) benumreinigi und gefärbt. Sach Der Struftur läß̈t jïch Dichter, oolitfiffcher

*) Die wichtigiten Sofalitäten für Steinjalz nach frormationen georonet, jind (nach) Ereoner):

Bildungen Der

formation:

Эekstzeit:

Tertiär:

Sreide:

গura:

Seuper:

\section{Sotalităt:}

Wüjtenjalz Der Rirgijenjteppe, in Arabien, Orfile, Seejalz am Doten Mieer, am gropien Salz: jee (utah).

Carbona in Sajtilien, Mieliczla und Bodnia in Balizien.

Weft älijhye Solquellen.

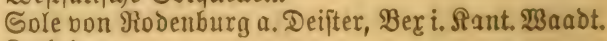
Sotfringen. 


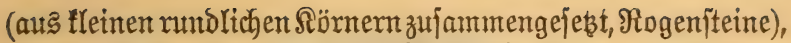

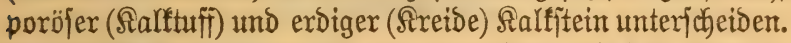

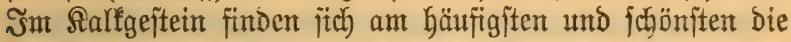
Beriteinerungen erbaltent.

Dolomit. Berjohiedenartige Mijabung von fohlenjaurem

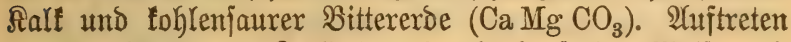
meniger bäufig als Salffitein, aber in Derjelbent Meije und mit biefent verbunden. Die poröfen zelligen ßarietäten Geižen

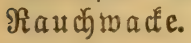

\section{Sulfate.}

$\mathfrak{A n h y b r i t ~ u n d ~ B i p s ~ ( w a j f e r f r e i e s ~ u n d ~ w a f f e r f h a l t i g e s ~}$ Salzitumjulfat, $\mathrm{Ca} \mathrm{SO}_{4} \cdot 2 \mathrm{H}_{2} \mathrm{O}$ ). Beise treten bejonders in ben tonigen Schichten auf und jind jtete Begleiter Des Stein=

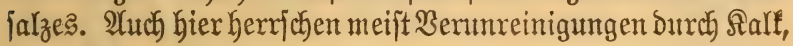
Ton, Eijen und Bitutmen bor.

\section{Pho Eppate.}

Die Pfosphate find int alfgemeinen felten, mur ber phos= phorjaure Salf ober 2 patit $\left(\mathrm{Ca}_{5} \mathrm{Cl}\left[\mathrm{P} \mathrm{O}_{4}\right]_{3}\right)$ tritt in Den meiften Bejteinen als afzefforijcher Bejtandteil auf, und ift in eitzelnen Bsegenden als \$hosphoritgeiteit bon grof̈em tecthijuten $\mathfrak{S}$ erte.

\section{Formation:}

Sofalität:

Muichelfalf: Dberes und unteres Rectargebiet, Ermitrall und

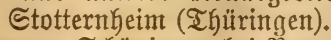

Buntiandftein: Seannober, Schöningen bei ßraunjabmeig. 3echftein: Bei Éera, Staß̧furt, Şalle, Sperenberg, Segeberg (S.olftein).

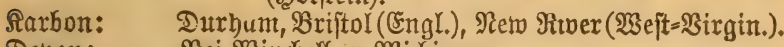
Devon: Bei Binchell tn Michigan.

Silur: Weft=ßirginia, Staat Mew Yorf, Saginaw in Mi-

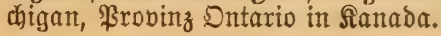




\section{Sirifiate.}

Die auperordentfich formenreiche (Bruppe Der Silitate ift bon bejonderer Bebeutung für Die gemengten Befteine, ba bie meiften Minteralien ber bulfanifjen (S) iteine zur (Bruppe ber

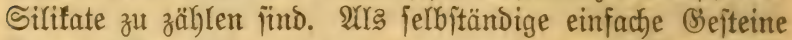
fönnen wir bezectchnen: S2ornblendefchiefer, bäup̈g in Den friftallinifochen Schiefergejteinen; Âtugitichiefer, wozu aud

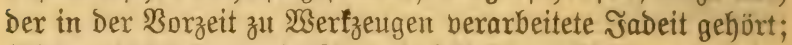

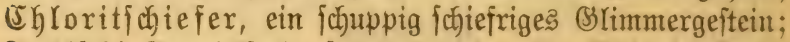

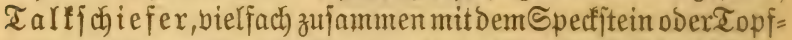
ftein borfomment, uns Serpentin, ein mafjerbaltiges Mag=

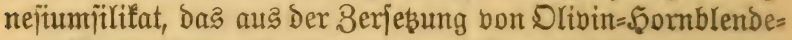
ober 2Yutgitgejteinen herborgegangen iit.

\section{Roflengefteinte.}

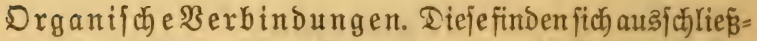
lich in Den fesimentären Frormationen und find aus ber Berjebung und Bertohlung von tierifchen und pflanzlichen Überrejten entītanden. Die organifchen $\mathfrak{B e r b i n d u n g e n ~ t r e t e n ~}$ entweder als Soglentwafferftoffe (Betroleum, Raphtha,

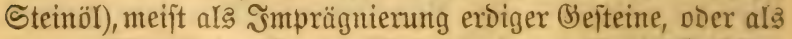

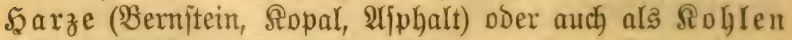
auf. Die Sohlen entītehen aus \$flanzen, unb wir tömen beren Bildoung an Den Torfmooren Keute noch beobachten; je älter bie Roblentablagerungen finb, um fo feîter wirid das (Beîtein und um fo gröper Der (3)ebalt an Siohlenjtoff. Die größ̈ten Rohlenablagenungen treffen wir in Der @teintohlenformation. Nach Dem (Sehalt an Siohlenitoff, Der Jejtigfeit und Dem 2flter

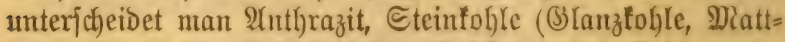

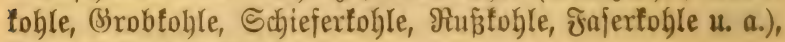
Brauntoble und Torf. 
Einfache Befteine.

\section{Bufammenjtellung}

ber Gauptfächlichen Unterjobetbungsmmerfmale (nach Creoner):

\begin{tabular}{|c|c|c|c|c|c|c|}
\hline $\begin{array}{l}\text { Sroblen= } \\
\text { Geiftetr }\end{array}$ & 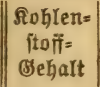 & \$ã tate & $\begin{array}{l}\text { Spez. } \\
\text { ઉ̋ervidit }\end{array}$ & Strín & $\begin{array}{l}\text { Crrwärmte } \\
\text { Rafltauge wirb }\end{array}$ & Brennbar \\
\hline $\begin{array}{l}\text { Braun= } \\
\text { fohle }\end{array}$ & $55-75$ & - & $0,5-1,5$ & braun & tiefbraun & Ieicht \\
\hline $\begin{array}{l}\text { Stein- } \\
\text { toble }\end{array}$ & $75-90$ & 2 & $1,2-1,5$ & $\begin{array}{l}\text { braun= } \\
\text { figwarz }\end{array}$ & $\begin{array}{l}\text { nidtt ob. hell= } \\
\text { gelblich braut }\end{array}$ & $\begin{array}{l}\text { z. Teil } \\
\text { leidgt }\end{array}$ \\
\hline $\begin{array}{c}\text { 2) } \\
\text { fit }\end{array}$ & $\begin{array}{l}\text { über } \\
90 \%\end{array}$ & $2-2,5$ & $1,4-1,7$ & $\begin{array}{l}\text { grauts } \\
\text { ichtwarz }\end{array}$ & $\begin{array}{l}\text { niçat } \\
\text { gefärbt }\end{array}$ & $\begin{array}{l}\text { mur bei } \\
\text { ftarfem } \\
\text { \&uftzuge }\end{array}$ \\
\hline
\end{tabular}

\begin{tabular}{|c|c|c|c|}
\hline $\begin{array}{l}\text { Roblen: } \\
\text { Beftetn }\end{array}$ & \multicolumn{3}{|c|}{ Erjcheinumgen beim Berbrennen } \\
\hline $\begin{array}{l}\text { Braun }= \\
\text { tohle }\end{array}$ & xuß̃ende Flamme & ftart rautchend & brenzlich finfento \\
\hline $\begin{array}{l}\text { Stein- } \\
\text { tohle }\end{array}$ & Gelle framme & ftart rauctento & $\begin{array}{c}\text { aromatijo bituminozs } \\
\text { riechendo }\end{array}$ \\
\hline 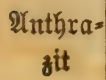 & $\begin{array}{l}\text { idroade oder gar } \\
\text { feine flamme }\end{array}$ & nicht rauchend & ogne (J)erudb \\
\hline
\end{tabular}




\section{Gemengte Gerteine.}

\section{Cruptiv= ober maffige Gepteine.}

\begin{tabular}{|c|c|c|c|c|}
\hline $\begin{array}{l}\text { Mame des } \\
\text { Seiteines }\end{array}$ & $\begin{array}{l}\text { Befentlide } \\
\text { Beftandtetle }\end{array}$ & $\begin{array}{c}\text { Struf }= \\
\text { tur }\end{array}$ & $\begin{array}{c}\text { Midfitge } \\
\text { alzefforifide } \\
\text { Beftandetie }\end{array}$ & $\begin{array}{l}\text { (S) fefteinse } \\
\text { barietäten }\end{array}$ \\
\hline geftei & & & & \\
\hline 1. Sranit & $\begin{array}{l}\text { Drthollas, } \\
\text { Sluarz, } \\
\text { (S)immer }\end{array}$ & förnig & $\begin{array}{l}\text { \$lagiollas, } \\
\text { Sernblende,Eor= } \\
\text { Dierit, Turmalin, } \\
\text { Magneteijen, } \\
\text { Titanit }\end{array}$ & $\begin{array}{l}\text { Biotitgranit, } \\
\text { Mustowitgranit, } \\
\text { Sranitit, Scbrift- } \\
\text { granit, ßegmatit, } \\
\text { Sranitporphyr }\end{array}$ \\
\hline $\begin{array}{l}\text { 2. Duarz= } \\
\text { Porphyr }\end{array}$ & $\begin{array}{l}\text { Duarz, } \\
\text { Drthoflas }\end{array}$ & $\begin{array}{c}\text { porplyy: } \\
\text { rifah }\end{array}$ & $\begin{array}{l}\text { Plagioflas, } \\
\text { Slimmer, Şorn= } \\
\text { blende, 2(ugit, } \\
\text { Magnetit }\end{array}$ & $\begin{array}{l}\text { Jelfitporphyr (ty= } \\
\text { pifdyer ßorphnr), } \\
\text { Felfitpechftein } \\
\text { (glafig) }\end{array}$ \\
\hline 3. Snenit & $\begin{array}{l}\text { Drthoflas, } \\
\text { 5ornblende }\end{array}$ & förnig & $\begin{array}{l}\text { Plagioflas } \\
\text { Biotit, } \\
\text { Magnetit, } \\
\text { Titanit }\end{array}$ & $\begin{array}{l}\text { Orugitinenit, } \\
\text { (slinmerinenit, } \\
\text { Syenitporphyr } \\
\text { (porphyrijd)) }\end{array}$ \\
\hline 4. Diorit & $\begin{array}{l}\text { Plagioflas } \\
\text { gornblenbe }\end{array}$ & förnig & $\begin{array}{l}\text { Slimmer, } \\
\text { Iugit, } \\
\text { Suarz, } \\
\text { 2lpatit }\end{array}$ & $\begin{array}{l}\text { Sormaler Diorit, } \\
\text { (Slinmerdiorit, } \\
\text { Duarzoiorit (To- } \\
\text { nalit), Porphyrit } \\
\text { (porphnrifd)) }\end{array}$ \\
\hline 5. Sabbro & $\begin{array}{l}\text { Bragiotras, } \\
\text { Diallag }\end{array}$ & $\begin{array}{r}\text { grob }= \\
\text { förnig }\end{array}$ & $\begin{array}{l}\text { Magnetit, Drivin, } \\
\text { (y)perithen }\end{array}$ & \\
\hline $\begin{array}{l}\text { 6. Diabas } \\
\text { (Briüntein) }\end{array}$ & $\begin{array}{l}\text { Flagiollas, } \\
\text { 2ugit }\end{array}$ & $\begin{array}{l}\text { förnig } \\
\text { (nteift } \\
\text { fein: } \\
\text { förnig) }\end{array}$ & $\begin{array}{l}\text { Magnetit, } \\
\text { Iitanit, 2rpatit, } \\
\text { Ortfollas, } \\
\text { Drivin }\end{array}$ & 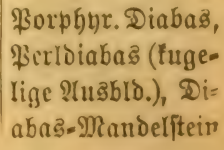 \\
\hline
\end{tabular}


Bemengte Bsefteine.

\begin{tabular}{|c|c|c|c|c|}
\hline $\begin{array}{l}\text { Rame bes } \\
\text { Befteins }\end{array}$ & $\begin{array}{c}\text { Befentride } \\
\text { Beitandtetle }\end{array}$ & $\begin{array}{c}\text { Strut }= \\
\text { tur }\end{array}$ & $\begin{array}{c}\text { 2Biditige } \\
\text { atzelforifide } \\
\text { Beftandeile }\end{array}$ & $\begin{array}{l}\text { (B) eiteins = } \\
\text { barietäten }\end{array}$ \\
\hline
\end{tabular}

\begin{tabular}{|c|c|c|c|c|}
\hline $\begin{array}{l}\text { 7. Mela = } \\
\text { phyr }\end{array}$ & $\begin{array}{l}\text { PIagioflas } \\
\text { Ulugit }\end{array}$ & $\begin{array}{l}\text { porphy } \\
\text { rifich } \\
\text { (blafige } \\
\text { (se. } \\
\text { iteine) }\end{array}$ & $\begin{array}{l}\text { Drivin, } \\
\text { Magnetit, } \\
\text { Upatit }\end{array}$ & $\begin{array}{l}\text { Diabasporphyrit } \\
\text { (ohne Dlivin), } \\
\text { Mandelfteine }\end{array}$ \\
\hline
\end{tabular}

II. ঐuing. Gruptivgefteine.

8. Irachnt

9. $\mathfrak{B h o n o}=$ lith

Drthotlas (Sanibin) por

\section{fỏrnig \$lagioflą,} Scornblende, porphy $=$ :

Sanidin, porphy. Rephelin

10. Bajalt \$lagio= tlata, Nepherin, ฉeucit mit $\mathfrak{A} u g i t$, Drivin, Magnetit

11. ミulta= MIas nijche ॐräị törnig Şornblende, und Biotit, porphy $=$ rijch Titanit, Şautn, Melilith, STpatit

glajigu. Beftanoteile porphy $=$ bom Irachnt rifch und Bhonolith Bimsftein (blafig). fornig),
Santointractgt

(porphyrifd), Duarztrachyt od. Siparit, $\mathfrak{A}$ ndejit (Itarf plagioflas. haltig)

Reucitopgyr

Dolerit (grob

friftallifiert),

IYnamejit (feitt-

Feldipatbajalt, Nepherinbajalt, Qeucitbajalt, Angitandefit (\$lagiollas und थ(ugit)

Sbjioian, ßectitein, 


\section{Sebimentärs voer geidjidjtete (jejteine.}

(Sriftallinifdye Sobiefergefteine.)

\begin{tabular}{|c|c|c|c|c|}
\hline $\begin{array}{l}\text { Same bes } \\
\text { Eefteins }\end{array}$ & $\begin{array}{l}\text { פBefentlide } \\
\text { Beftanotetle }\end{array}$ & $\begin{array}{l}\text { Struf- } \\
\text { tur }\end{array}$ & $\begin{array}{c}\text { 2stditige } \\
\text { atzefiortiche } \\
\text { Beft anbteile }\end{array}$ & $\begin{array}{l}\text { Befteins. } \\
\text { barietäten }\end{array}$ \\
\hline
\end{tabular}

1. Brneis Drthoflas, flajerig Branat, Branitgneis,

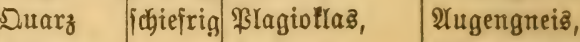
Ślimmer Cordierit, (Biotitund Magnetit, Musfomit)

Turmalin,

đ̧raphit, Sornblende

2. Orant= Jeldipat, förnig Biotit, lit

3. $\mathscr{A} m p h i=$ 5ornblenbe bolidicfer

4. Eetlogit

5. BYİ̈merjobiefer Juarz, Siranat

Gis wohlge. ichichtet

jodiefrig

Turmalin,

Eyanit,

Diallag

Suarz, Sranat, Biotit, Magnetit

Ssranat, 2lugit $($ Dm $=$ phacit)

sslimmer, Duarz

6. \$hnllit (Utrton= ¡diefer) Cfylorit, grob= \$slimmer, Şornblente, Duarz, Eyanit fein= Sranat, ichiefrig Turmalin, Talt, (E)lorit, Magnetit fein= Ehiaftolith, ichiefrig Turmalin, 5ornblente Magnetit
Brimmer, Duarz, Felojpat, Rutil
(S) Yimmergneis, \$ornblendegneis, Cordieritgneis, Braphitgneis, Sericitgrei3

Biotitgramulit, Diallaggramulit

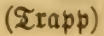

Strabliteinjăiefer

\$ranatiela

(5ranatidjiejer, Chloritidiejer, Ialtjøiefer

\$hyllitgneis, EGiajtolithjojiefer, frlectenidiefer, Wesidjiefer 


\section{Crümmergesteine (f́lajtijche Sseiteine).}

\section{Bulfanifde Trümmergefteine.}

1. Tuffe find bie bet ben Emutionen auggetworfenen Maffen, weldje Durch Wafjer zu Sd)lamm zujamntengebadfen

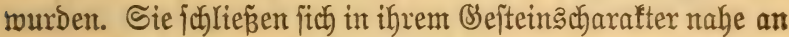
bie betreffenden maffigen (Befteine an. S2ierber gebört: \$or. phyrtuff, Diabastuff (હrïnjteintufi und Eschalitein), Trachyt tuff, Bajalttuff.

2. $\mathfrak{B u l f a n i j h e r ~ S c h u t t . ~ U n t e r ~ D i e j e r ~ B e z e i c h n u n g ~}$

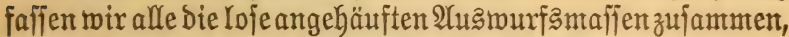
weldje fict bejonoers bei ben jungen und Den noch tätigen

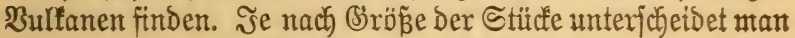

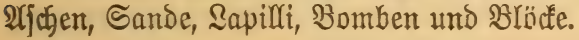

\section{Neptunifjue Gsebilde.}

3. Tongefteine. Dieje umfaffen bie Unmand humgs= und Berfekung sprodufte aus anderen, bejonders den ferbjpatreichen (3e) iteinen. Sie zeichnen fich alle Durch ein erbiges gleithy

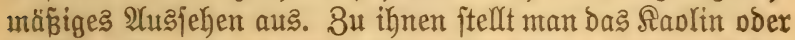
die \$orzellanerbe; Den Tomfchiefer, Schieferton, Ton, Rehm

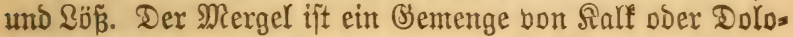
mit mit Ton.

4. Sanditeine bejtehen aus Duarztönten, weleje burch

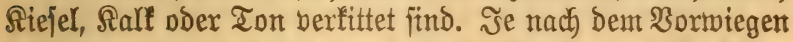
bes einen oder anderen Beftandteiles fpricht man von tieje ligem, falfigem, tonigem, Dolomitif(bem $\mathfrak{u} \mathfrak{i m}$. Santifein.

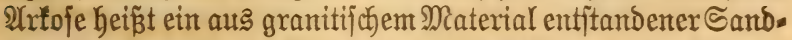

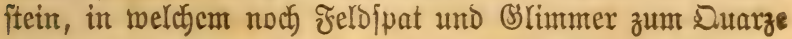
treten.

5. Songlomerat nonnt man ein Brejtein, weldes aus berfitteten abgerollen rumben Bsejteinsftitufen gebildet ift, toährent bei ber 
6. Breccie idjarffantige effige Butchitüde bortwiegen.

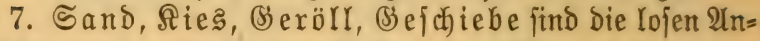
Gäufungen ber Durcty Dą $\mathfrak{B a f j e r ~ o D e r ~ ( E i ̉ ~ z e r t r u ̈ m m e r t e n ~}$ Bejteine.

\section{II. $\mathscr{A}$ b $\{$ d nitt.}

\section{Die Entitehung der Geiteine.}

( Betrogenetijaje (Geologie).

\section{A. Butfanijde $\mathfrak{B}$ ilbuttgen.}

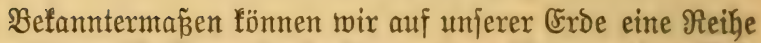

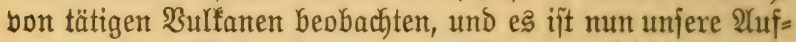

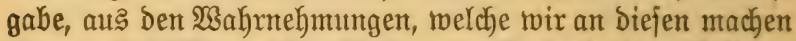
tönten, Schlïffe auf Die Bildungsmeife Derjenigen Beiteine zu ziełen, welche bie größzte Ânalogie mit Den Erntptiomafien ber $\mathfrak{B u l f f a n e}$ zeigen, und melche mir baher als (Exuptingeiteine bezeidinent.

Wir gehen, wie ichon angedeutet, bei unferen Betractungen bon bem aus, was uns bor Âgen liegt, und futchen

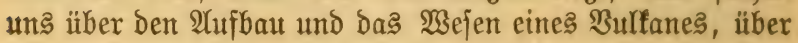
Die Eruptionen, bie fie begleitenden Erffeimungen und ibre

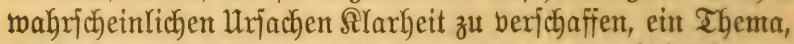

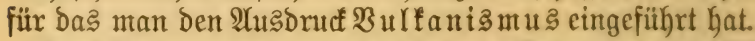

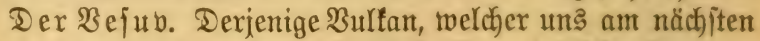
liegt unt burch feine flaren Berthältniffie und Yeichte Bugäng=

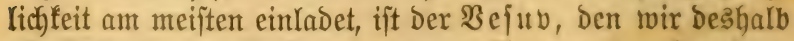

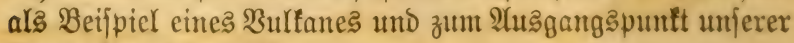
Beobactutungen wäblen.

Die ganze Begend atm Reapel billdet cine groß̌e CEbene, bie fith) nur mentige Meter über bas Mieer erhebt und weldhe bon

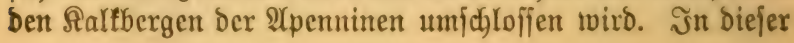


(8bene erbeben fich eine Reibe bulfanifcher Berge, bie in zmei Sruppen zerfallen, Der Bejub im Sübojten und bie \$hlegräifichen Zelder im Norbmeiten bon Neaper; Die gänze Ebene ijt bedecft bon bulfanijchen 2 (j) Schlamm zufammengebadfen, ein feites S'ejtein, jogenannten

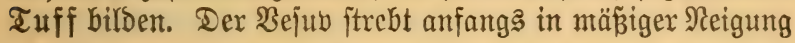

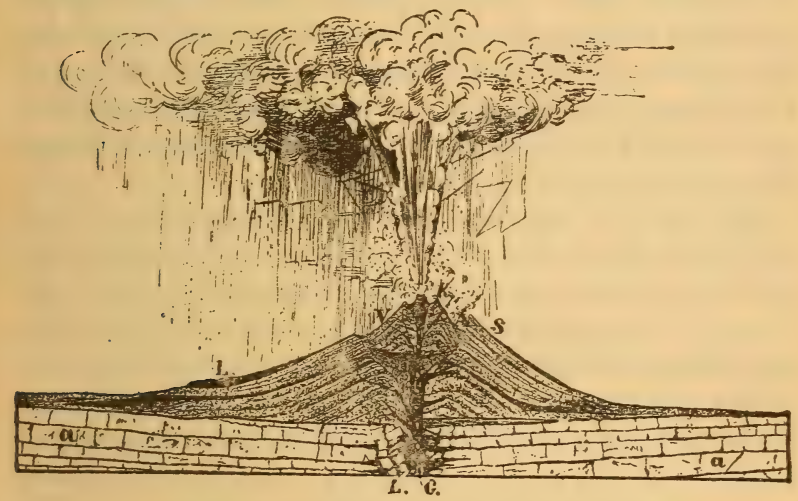

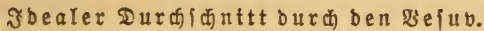

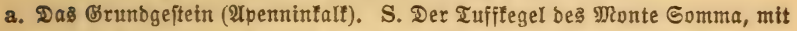
alten \&abaftrömen. A. Das Atrio del Cavallo (alter Sommafrater). V. Der 2rïjentegel bes Bejub. K. Der Bejubfrater mit bem Eruptionstegel (Bocca). C. Sanal mit emporbringender Eruptibmaffe. L. Sabagänge unb Ströme.

empor, wird jedoct immer fteiler, bis wix bas ßinggebirge erreichen, bas namentfict int Norben ala Mionte Somma noch wohl erfalten ift und bis zur Şöhe von $1124 \mathrm{~m}$ emporíteigt.

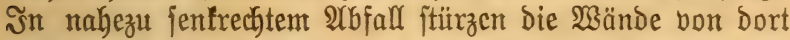

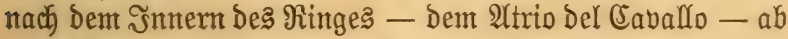

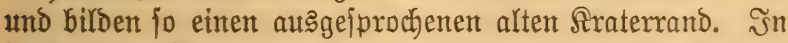
biejem alten Srater Der Somma erbebt fich mun fteil anjtrebeno 


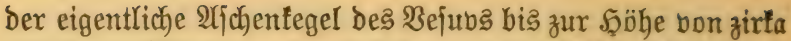
$1300 \mathrm{~m}$. Dben angelangt, feלhen mir zunächit mieber einen

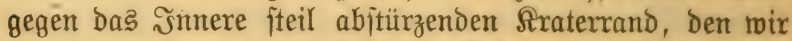
überfteigen, um uns fchließ̧lich Dem fleinen eigentlichen (Enup= tionategel - der Bocсa - zu nähern, welcher wieder mit

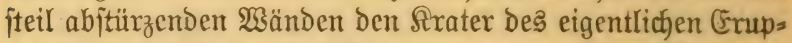
tionaheroes bildot. $\mathfrak{2}$ us ignt werden in rubigem 8uitande getwaltige Dampfmaffen uno von Beit zu Beit Rababoutben

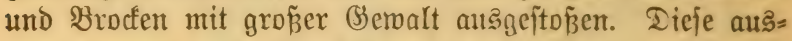
gemorfenen Schuttmafien falfen teils in Den Sirater zurïd, teil's aber auch auf bie Bocca felbit Kerab und tragen fo immer zur

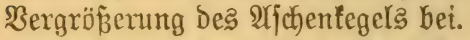

So fehen wir am $\mathfrak{B} e$ jto brei Berge fozujagen ineinander= geichachtelt, beren jeder einer verfajiebenen (Exuptionstätigleit bes Berges entfpringt. Ter $\mathfrak{B}$ ejuv der $\mathfrak{A}$ (rten bis zum Jabre 79 n. (Shr. war bie Somma, welche einen bulfanif chen Berg mit mäpigem Srater Darjtellte, in Dem fitch ein fleiner See

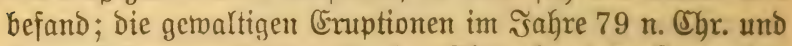
in Der folgenden 3eit warfen den Iffchenfegel im Sirater ber Somma, Den Bejub, auf, Deffen gegen $500 \mathrm{~m}$ breiter frrater

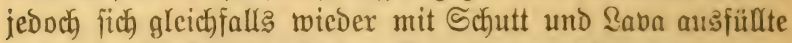
und in Den bie Geutigen fleinen Eruptionen wieber einen neuen âfictenfegel mit frater aufgemorfen haben.

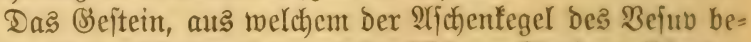
fiteht, ift ein Sraufmerf bon lofen 2 jichen, Sanden, Bomben

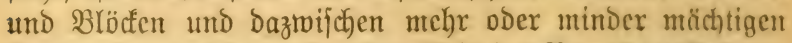
¿avamaffen, welche meift auf der Seite brs Berges auştrömen

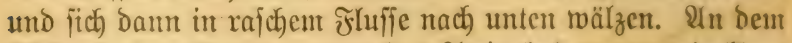
Irbbruche Der Somma gegen das 2Atrio befonmen wir flare Profile Durch einen Teil bes Sberges; ; wir fehen ben ganzen Berg auts fchalenförmig ïbereinandergelegten Iecten bon feit berbacfenent $\mathfrak{Q}$ (j) jebe einer Eruption bes Bergess entfpringt; in Dem ?tufriffe 
zeigt fich un' baher dą Bild einer ausgeprägten Schichtung. Maffenhaft merben biefe Tuffbänfe Durchbrocken bon ฉaba= gängen, welche fich bann Decfenartig zroijchen Den einzelnen Schichten ausbreiten.

A̛ fbau eines $\mathfrak{B}$ ulfanes. Sombinieren mir alle dieje Beobachtungen, fo tönen wir uns leicht einen ibealen Durch)=

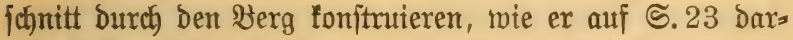
geitellt ift. (5ine Burchlinie im Untergrunbe hat den bulla nifden Maffen Belegenheit geboten, aus ber Tiefe emporzu=

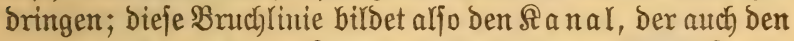
ganzen aufgemorfenen Regel Durchfest und mit Dem Srater endigt. Die bulfanijchen Maffen, weldye ausgeworfen werden, find bexfdiedener Satur; zunächit haben wix ungebeure

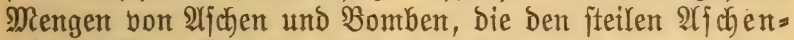
fegel bilden. Nach Dem (Befeb Der Schmere fondern fich bie hoch emporgejchleuderten Miaffen beim Scerabfallen jobichten= reife in gröberes und feineres Material, und da eine Fruption

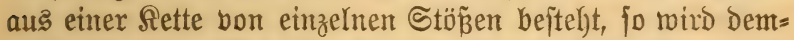
entiprechend ber alf chentegel aus wohlgejchichteten \&agen auf= gebaut fein. Bugleich mit diejen trodfenen Bejteinsmafjen find aber Die (Enuptionen von grof́zartigen Tampfentrictlungen

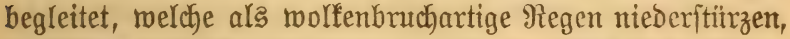
die 2 (j)hen in Schlammitröme ummandeln und zu Tale führen; nach Der Eritamung bilben dieje die bulfanifacen $\mathfrak{T} u f f e$,

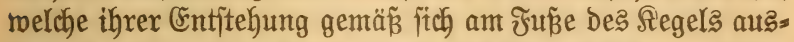
breiten und zu einer Berflachung Des Berges fübren. Dieje

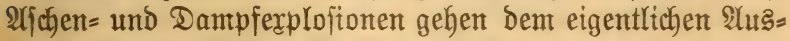
bruch ber feuterflüfïtgen Maffe, Der $\mathfrak{a b a , ~ b o r a n ; ~ f i e ~ f i n d ~ e s , ~}$ welche gleichjam Den Schlot gereintigt haben, in welchem die faywere Maffe nach oben fteigt. In Dex Regel erreicht jedoch bie Lava den firaterrand nicht, fondern zerjprengt jeitlich ben 2rjofen $=$ und Tuffmantel und bricht mun an ber Seite des Berges aus. Erft ift die Sabamaffe jefr dinnfliifitg und jobiest 
mun mit rajender C(f)nefligfeit als $2 a b a j t r o m$ ben Berg Ginab, aber felre Gald wirft bie Albfühlung auf fie ein und als zäfyer Teig twälzt jïch Der Strom träge meiter, immer bon meis teren nachjichiebenton Mafjen gebrängt und ïberflutet. Šit Das Terrain giumitig, fo breitet jich Der Strom aus und birldet

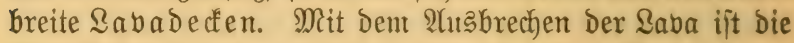
Geroult Der Cruption erfictöpft und es tritt meijt eine lange Bauje ein, in melcher die Beritönung Des gebildeten Bulfanes Durch bie Berwitterung und $\Re$ egentwaffer rafd um fich greift.

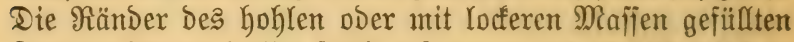
Sanales ftürzen in fich ferbjt zujammen umb es bild bet jich fo ber fteil nach immen abfallende $\Re$ raterrand. Die inmeren geftauter Berbäfjer Durchnagen Käufig Die Siratermanto, in tiefen Safluchten (Barranco) miro Der loctere Boben zu Tale

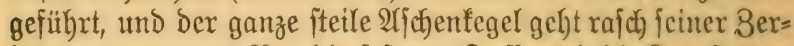
ftörung entgegen. Nur Die feîteren Tuffe und Die \&abajtröme leiften mehr $\mathfrak{B i d e r j t a n d . ~}$

Da folgt eine nette Crnuption; bie ganze Maffe, welche ben Sanal beritopft hat, wiro mit groferer (Semalt wicber hinaus= gefchleubert, neue 2(jchen aus Der Tiefe fommen Dazu, und bald erbebt fich im alten frater ein neuer Bulfan und neue

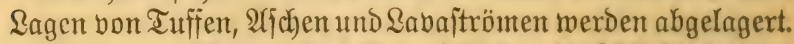

Stratoburfante. Tas Snorefultat ergibt fdjliéslich einen

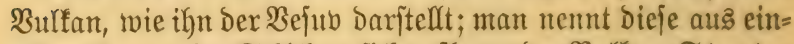
zelnen Sagen ober Schichten fich) aufbautenden Bulfane S trat o= burlf́ane ober gejobichtete Bulf̈ane. Şhre Entítebung ver= banfen fie ben unterbrockenten (Eruptionen, welche begleitet uno herborgentfen find Durd Explofionenen bon $\mathfrak{W a f f e r =}$ bampf. Der größ̄te Teil ber jeß̧t noch) tätigen Bulfante ge= Gört zu Den Stratobulffanen und es ift eine nicht zu über=

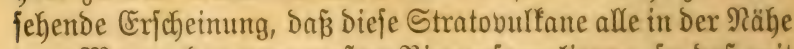
bom Meere oder yon groß̋en Bimtenfeen liegen, fo daß̃ mit

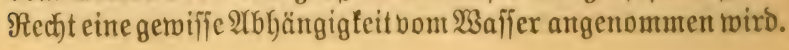


Die Berjtönung Der aus locferem Material aufgemorienen Stratobulfane geht fo rajch vor f̈̈ch, Dañ wir ung nicht rundern Dürfen, menn diefelben uns aus früheren Fro perioden nur in geringer $\mathfrak{U}$ nzahl erbalten find, twas jedoch

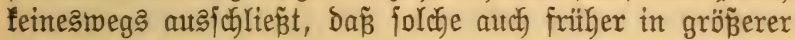
Menge borbanden waren.

Mafjige $\mathfrak{B u l f a n e . ~ D e n ~ g e f d i c t e t e n ~}$ Bulfanen gegen= über ftehen die majjigen $\mathfrak{B u l f a n e . ~ D i e j e ~ e n t i t e b e n ~ g e = ~}$

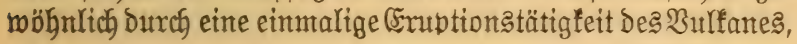
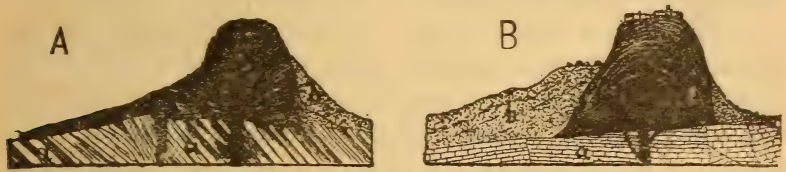

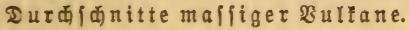

A. Bafaltuppe unb Bajartbede mit Gäulenabionberung. B. Fhonolitblegel

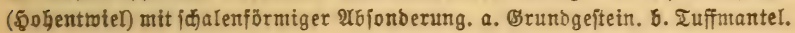

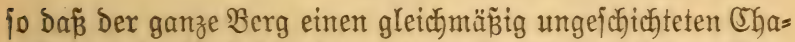
rafter zeigt. SUuch hier beginnt bie (Fruption ntt Dem $\mathfrak{A} u s=$

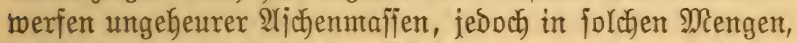

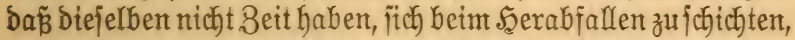
fondern einen ungejchichteten Tufffegel aufwerfen, in Dem grobes und feines Material regellos aufgehäuft ijt.

Die হabamafien, weldye im Ranal aufitetgen, find jeboch nicht Dïnnflïfïg, fondern Dicf breiartig, auch ît ifre explojtoe Sraft eine mur geringe. So fommt es, Dẫ fie mur felten Den Sraterrand überftrömen, DDer feitlich ausbrechen und fich Dann in Strömen und Ded e n ausbreiten. Sieröbultich $\mathrm{er}^{\mathrm{r}}=$ ftarren fie jobon im Sanale jelbit und billden jo (S) änge im Sejtein, oder f̈e erreichen Die Dberfläche und türmen fitch Dann zu Dombulfanen und \{uppen auf. C5s ift dies eine für

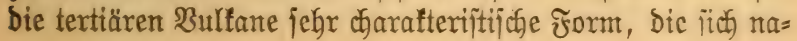




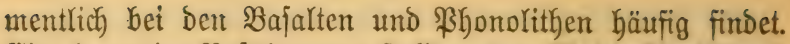
Eine Derartige Bajaltgruppe ftellt uns meift bie 2A DeE bebeuteno erweiterten Siraters Dar, wobei Der Tuffmantel

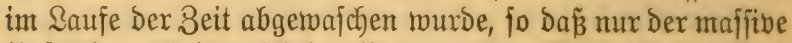
Bafalttern uibrig gebrieben ift; zumeilen ift es aber auch zu gar feiner $\mathfrak{A}$ aizenenuption gefommen uns in biejem Falle murbe bie ganze Maffe des Tombulfanes als zähe Cruptio= maffe herausgequeticht und aufgetïrmt, wie jich bies z. B. autch bei Der Yeßßten Eruption Des Miont Pelé auf Martinique(1902) an bem jogenannten "Cone“ beobacten Yieß̃.

Nicht inumer Drängen bie bulfanifchen Maffen bis zur Dberfläche, fondern bleiben zumeilen auch zwifhjen ben Schichtengeiteinen eingczmängt itecfen. Terartige in ber Tiefe exftarrte $\mathfrak{B u l f a n e}$ murben befonders bäufig in $\mathfrak{B}$ eĩten bon

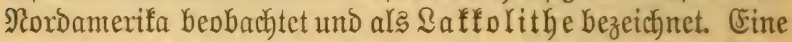
ähnliche (Erficheinung läp̧t fich aber auth bei biclen unjerer alten bulfanif̧chen Mlaffen (ङranit, Diorit ujw.) fejtitellen uno erflärt Deren grobförnige Stmuftur.

Maare. Eine weitere, bejonders in ber Fifel und Siid =

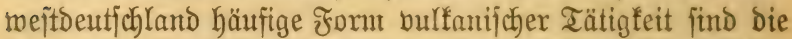

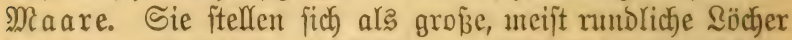
bar, bie bielfach jest mit $\mathfrak{W a f j e r}$ angefüfl jind, und rïfren bon einer gewaltigen einmaligen Explofion her, weldter feine weitere bulfanifache Tätigleit nachfolgte. Dem Miare ents fpricht nach ber Tiefe zu ein "Schuff́fanal", Der teils mit aus= gemorfenen $\mathfrak{A}$ (j)hen, teiłs mit bon Den Seitenwandungen Gereingefallenem Material exfüllt iit.

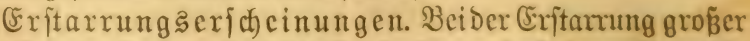

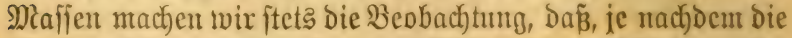
Daffe rajecher ober langfanter abtiiglt, eine berfofiedenartige Struftur entiftegt und zroar Derart, Dá̉ bie Ctruttur um fo feintörniger wiro, ie feffueller bie Erftarrutg erfolgt. So fommt

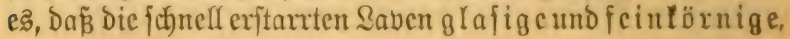


bie grop̈en bulfaniforen Suppen porphyrijdye ober aud förnige Sefteinsausbildung aufmeijen.

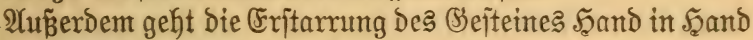
mit einer 8 ujammenziehung Der ganzen Mafje, welche zu einer fonzentrijchen Schalenbildung fïlyren fann, wie roir bies am fabjuten bet Den \$honolithen jeben. Dagegen zeigen die Bajaltberge häufig eine jehr verjchiebene Stmuftur. Wir finden

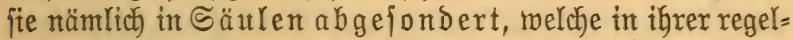

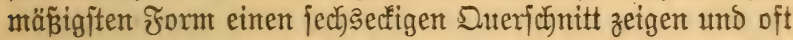
auf Das zierlichjte ausgebild et find. Die Säulcn find im grop̈en ganzen rechtroinflig zur Ulb fühlung

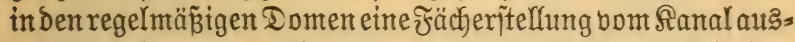
gehend befommen, wäbrend fie in Den Iecten bertifal ftehen. Dieje Säulenabjonderung bei ben bulfanijacten Mafjen ent= fteht gleichfalls bei ber rajchen Crritaming bes Ssejteins an

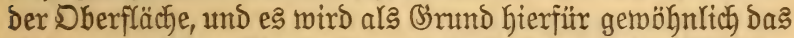
Bujammenziehen (Rontraftion) Des Befteins beim Orbtithlen angejeben; andrexjeits mird aber auch auf bie 2Uusbehnumg (Expanfion) Gingetwiejen, welche in Dem Momente eintritt, wenn geidymolzene Maffen in Den feiten Bujtand übergehen, und welche gleichfalls zu einer 2 (bjondemung in Säulen führen fann.

Sff̈enbar tritt die 2 bfondenung in Säulen nur bei folchen (Seiteinen ein, welcherajch und ohnebedeutenden Druct Darüber

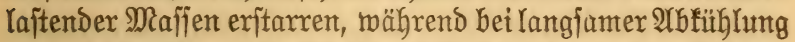

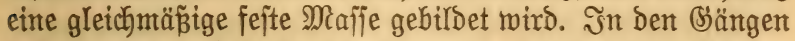
bejonders der trijtallinifch törnigen (Sejteine läpt jitch be=

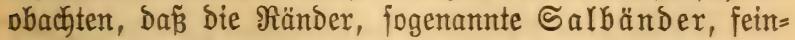
fömtg find, während Das (sieftein nach inten zu immer grob= fömigere uno pegmatitijace Stmitur annimmt. Ferner tritt bet manchen alten bulfanijchen Bejteinen, bejonderß bei Den Dia= bajen und Dioriten, noch eine fugelf p̈rmige $\mathfrak{A}$ b j ond erung

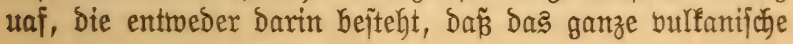




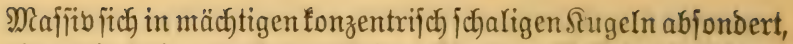
ober wir finden auch mur in bem förnigen (jeitein, Das bann gleichfam bie Binunomaffe bildset, bie Siriftall=(5)emengteile in 尺utgelform gruppiert.

Einteilung ber $\mathfrak{B}$ ulfangeiteine. $\mathfrak{B}$ ergleidyen mir bie in Der Erbe auftretenden bulfanifcten (Sciteine miteinander, jo Yäp̧t fich beobachten, Daß̧ bie tertiären Bajalte, \$honolithe und Tractynte jich am nächiten an bie rezenten Saben an= fch)ležen, ebenjo wie bie fie begleitenten Tuffe eine analoge Bildoungsmeife anzeigen; wir bezeichnen jie Daher als neo=

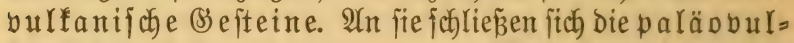
fanifchen (Se feine (Duarzporphyr, Melaphyr und Diabas) an, welche gleichfalls durch porphyrifche 24usbildung, (3las= bitsung und Begleitung von Tuffen Berwandffchaft mit ben

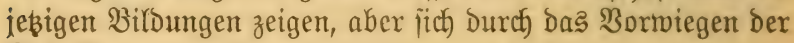
Sitejeljäure auzzeichnen.

Diejen echt bulfanifchen Biejteinen ftehen bie jogenannten

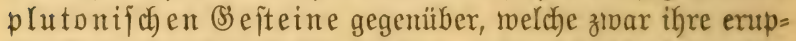
tive Natur Durch biele Merfmale berraten, aber Doct) von Den

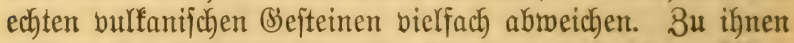
gefören bie Branite, Sycnite, Diorite und Babbro. Âfen

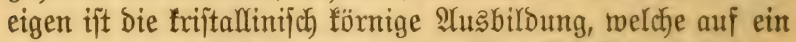
jehr langfames Eritarren finmeipt. Man ift bafer geneigt ant

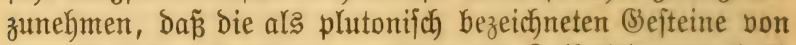

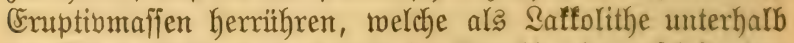
Der Erobberfläche unter Dem hohen Dnud Der Darauj lajtenden Sctyidyten ober in Der Beit Der archäifachen Formation unter unbefannten atmofphärifichen Berbältnififen febr langfam er=

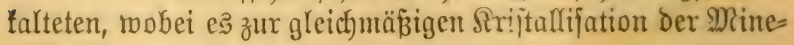
ralien formen formte.

Begleiterjdeinungen ber Bulfane. Wir baben nod einiger anderer gcologif(c) widftiger Fattoren zu gedenten,

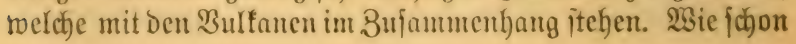


bemertt, find bie bulfanijchen Enuptionen begleitet bon ges maltigen Dampf = und Basexplofitonen; Diefe Basausitrö= mungen Dauern noch lange nach Den Fruptionen fort und merden als Solfataren, Mofetten oder Jumarolen be= zeichnet, je nachdem es Schwefel=, Rohlenjänte= oder 2 Sajןer dämpfe find, welche ausftrömen.

Somohl die bei Dem Empordringen Der glutflïjïgen Maj̈en

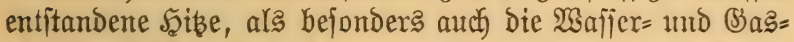
bämpfe bleiben natïrlich nicht ohne (sinmirfung auf bas

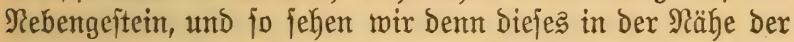

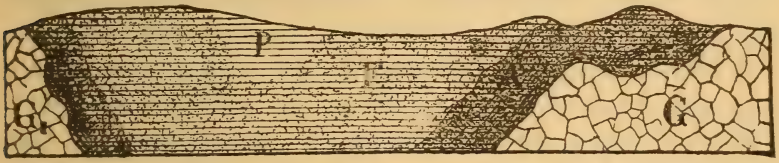

Rontaftzonen bei હaneeberg im Erzgebirge.

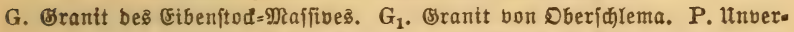

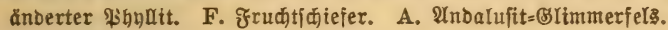

Fruption引herbe gemöhnlich umgemandelt. Man nennt dieje

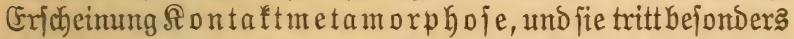
føön bei ben alten (Emptionaffen auf, welche wix als pluto=

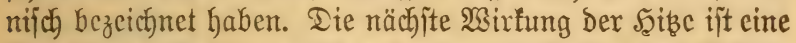

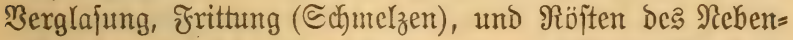
gejteins, mobei fitch Diejes häurig mieder in Säulen abjondert, rie die Sejtelliteine eine 5 Schofons; Sandftein wird gefrittet, Braunfoble wird zu Steinfoble bertolt und Salfitein in Mar= mor ungeroandelt. Noch ausgedehnter und tiefgreifender f̈ut Die Wirfungen Dor heî̉en Iämpfe, welche Die Sontaftzonen

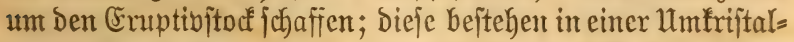
lifterung und Untmandlung Der Stultur Der Nebengeiteine.

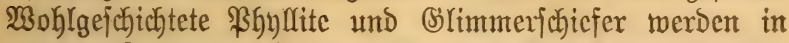
zonentweife um bas Exuptiogeitein gelagerte Âtoalujitfelfen, 
SGiajtolityjubiefer, Frucht= und Snotenjobiefer umgemandelt, und allentbalben treten in Den Rontaftzonen neue Mineralien auf, melche fpäter mieber vom Waffer verarbeitet uno in mädd. tigen $\mathbb{E} r z=$ und Mineralgängen abgelagert merben tönnen.

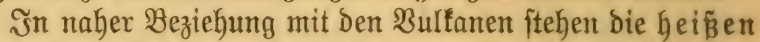
Duellen, Beifer und Schlammulfane. Sie alle find gebildet Durch heiź Waffer, welche aus bedeutender Tiefe emporbringen und infolge ifrer Saize eine Menge Mineral.

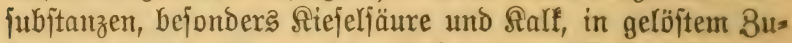

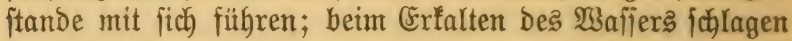
fie fich nieber und fübren zu jogenannten Sinterbiloungen

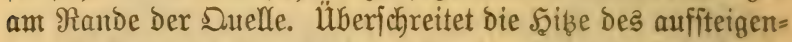
Den Saffers nicht ben Siedepuntt, ober ijt Die Duellröhre fo

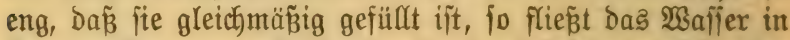

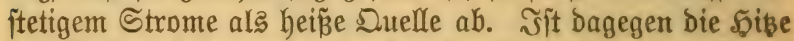

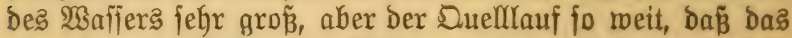
oben ftehende $\mathfrak{B a f f e r}$ abgefithlt wird, fo gerät ber Duellauss flus in Stoctung, bis bon unten fo biel size zugefübrt ipt

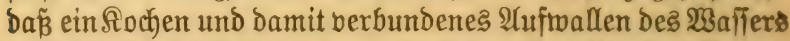
entifteht; Dies füfrt zu einer plößlichen Eruption Der Duelle, und man nennt Daber bieje (5xicheinungen Springqueflen ober Breifer. Bei ben Schlammbulfanen miro mit (bas geichlwän= gertes $\mathfrak{W a f f e r}$ zufammen unit Schlammmaffen ausgeworfen, lebtere jtammen jeboch mur aus bem tonigen und leicht löహెs lichen Nebengeitein.

\section{Cheorie der Vulkanbildung.}

Bum Schluffe haben wir aud noch einen Bridf auf bie Sypothefen inber ben Urjprung und bie Entitehung ber bulfanifchen Tätigfeit zu merfen. Sian hat bes

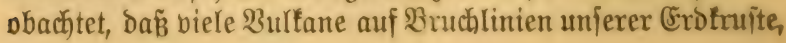


jogenannten Bermexfungşpalten, liegen, weldye, twie twix ipäter jeben merden, die Erbe allenthalben Durchieben. Şiex=

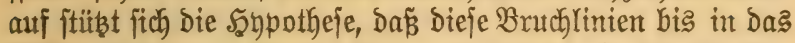

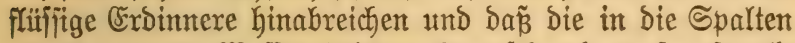
eingebrungenen $\mathfrak{W a f f e x ~ ( r o i x ~ m a c h t e n ~ j o h o n ~ b a r a u f ~ a u f m e r t = ~}$ fam, Daß̧ Die jeß̧t tätigen Bulfane vielfach an Meere liegen) bie Explofionen Gerbeifülyren. Dieje Theorie ift jedoch nicht anzuerfennen, Da meder die Spalten und Brïche, noch biel mentger bas $\mathfrak{W a f f e r}$ in foldbe Tiefen einbringen tann.

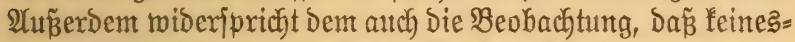
megs alle Bulfane an Spalten gebunden fint, fondern Dañ es eine jefr grop̈e Menge bon foldhen gibt, bei welchen ein $8 \mathrm{u}=$ fammenfyang mit Spalten nicht nachzutweijen ijt.

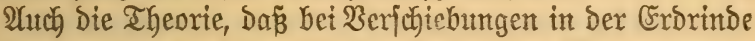
Wärme exzeugt werbe, welche die Sejteine zt Magma unzu= ichmelzen imftande ift, ober diejenige, Daj̃ Durch die Spalten

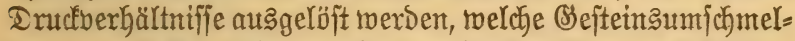
zungen mit fich bringen, hat wentg fiur fich und fteht mit

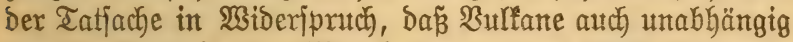
von Spalten auftreten. Dan hat neterbings (Stübel) die buts fanijoçe Tätigfeit aus Der zentralen glutflitfïtgen Magmamafie in peripherijche Szerbe verlegt, welche gleichfant als Relifte in Der Erftarmughlinite übrig geblieben jein jollen, und bie Urjactje Dex Explofionen wird teils in Der Berüfrung mit

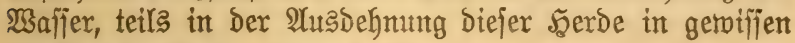
Stabien Dex Erjtarmung gejucht.

\section{B. Die Sebimentär=GSefteine unt ifre Biloung.}

So mannigfaltig uns auch Der Sharafter Der bulfanifchen Seiteine entgegentritt, fo bilden bieje boch einen nur febr teinen Bructeil in Der 8 ufammenjebung Der Erorinde 
und werben weit übermogen bon Den Sebintentärgejteinen. Wie fatjon in ber tabellarifchen überjictst angegeben, finden wir unter ifnen fotwohl einfache (3e)itcine, wie Ralf, (sips, Stein= jalz, Sohle, als auch gemengte (Siciteine - jog. friftallinifidse

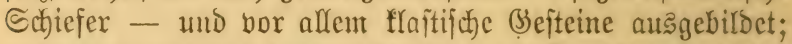
unter Yekteren find bejonders bie Tone und Sanditeine widytig.

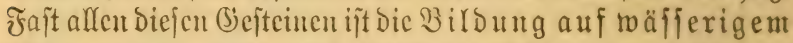
Wege eigen, weldye fich Durch bie Arblagerung in ge=

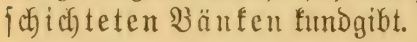

\section{Tätigleit bes $\mathfrak{B a f f e r s . ~}$}

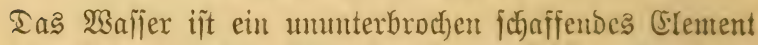
auf Der ๔roe, und jein Bejtreben ift, möglicbjt auझzuebnen. Ununterbrochen berarbeitet uno zeritört es bie feitlanosmajien unt lagert fie wieder in ben tieferen Ragen, befonder: ben Meeren ab. Dieje Beritönung geht teils auf chentifchem

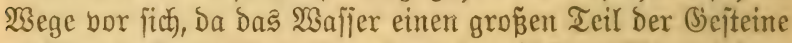

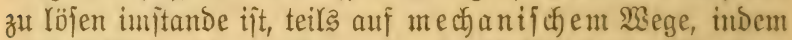

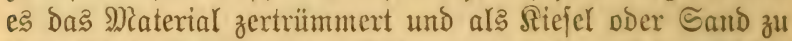
Tale füthrt. Bei Der ncuen Arblagerung fint meift forwohl bie

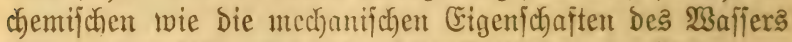

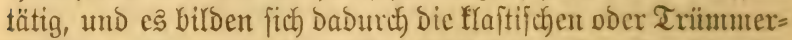

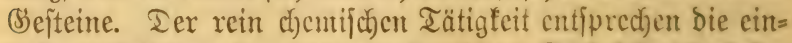
facten Beiteine, ber rein mect)antijecten Tätigłeit bie lojen Santo und sierölle.

\section{Sriftallinifde Schiefer.}

So ungezmungen und reidft fich auf biefe $23 e i$ e cine $^{\mathrm{E}} \mathrm{r}=$ flärung für Die flaftifichen und cinfachen Eedimentärgeiteine

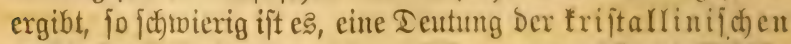

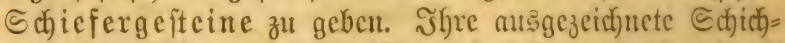

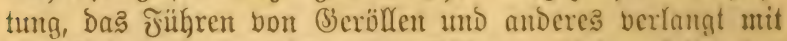
Entfodiedentfeit eine febimentüre Billoung. S(ndererfeits aber 
weidjen fie in ifrer 8 ufammenfeßzung aus berfojiebenen Mine= ralien fo jegr bon ben fpäteren Bifloungen ab und nälyern jich

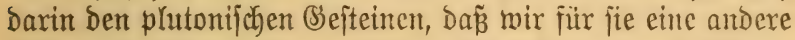
Bildungsiveife antelynten milffer. (5) mitroe zu meit füfren, auf Die bielen Sygpothejen einzugehen, welche Gierfïr aufgejtellt

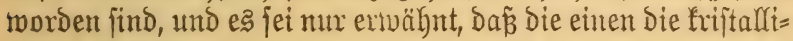
nifchen ๔chiefer als urfwrïnglich fo entitanden aumelyen, toobei

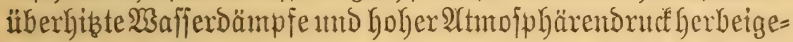
zogen twird (Diageneje). Die anderen Dagegen, und dieje Anjicht

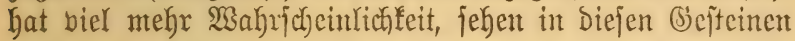
nur ein Untwandlungsproduft (Metamorphofe) aus normalen Schiefergeiteinen. 2tuch hierbei fpielt der Inut und Die Dabei

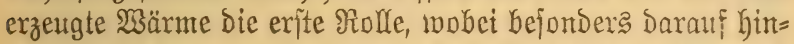

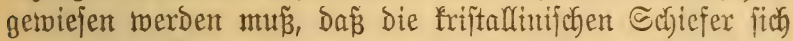
inuner in geitörten Ragerungen befinden, bei toelchen itarfe

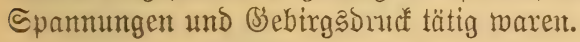

\section{Tätigleit Des Eifes.}

Wie das fliuffitge Wafjer, fo arbeitet autch das gefrorente Waffer ober (Ei beint Befrieren erleidet, zerfprengt es Das Bieftein, in Defien feine \$oren und gitfje es eingebnumgen ift. Gropartig tritt uns bie Tätigfeit bes Cijes bet Den (3) Yetfohern ent=

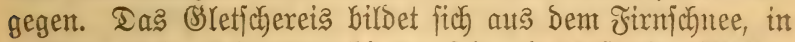

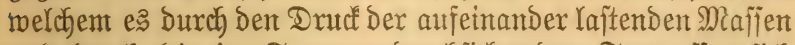
und Durch die in Sommer burchfiffernden Tagmaffer fith zut (Eis berfeptigt. Da fith in Den Schneeregionen Der $\mathfrak{B}$ erge

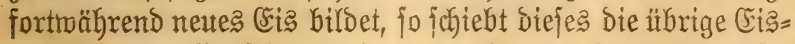
maffe - Den Blfetjcher - intmer weiter talabmärts. Diejer Cisiftrom arbeitet berart auf feinent lutergrumbe, baj ex fleinere Szindernifije abjobelt mid zu B̈eröll berarbeitet, und man bezeichnet biefe an Der Solyle Des Siletichers gebirbeten Seröllablagerungen als Şrundmoräne. Turch bie gegen= 


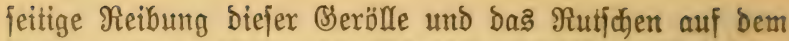
Itntergrumbe entitehen die Bsleticherfichliffe. Denfelben Borgang beobachten mir auf ben Seiten bes (STetichers; aurserbent lagern fich bort bie Scthuttmafien ab, welche yon Den Seiton Des Tales auf Den (Sletfcher herabjtïrzen, und bieje billoen bie Seitenmoränen. Wie bie Alrme eine?

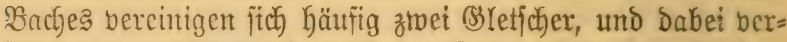
\{dymelzen natïrlich bie beiden zufammentretenden Seiten= moränen zu eintem mun in Der Mitte berlaufenden Beröll= Gaufen - Der Mittelmoräne.

Syentzutage finten wir in Der gentäsigten 8one bie (3) Tetficher mur noch it ben hörtfiten Berggegenden, twährento fie it Det Polargegenden noch tief herab, zun Teil bis zum Meeresfpicgel reichent. Setfiebt ein (siletjcher fich in bas Meer Ginein, fo zerbrödfelt ex und fofmimmt bann als (Fisberg, bon Der Strönung getrieben, weiter. Jnt biejen Begenden bejobränfen fich bie Błletfcher aber nicht allein auf Bebirgs= täler, fondern bebectent weite Strecfen bes Jlachlandes; ntan bezeicfnet foldye vismaffen als sntandeis.

Ia roir auch in Den gentäpagten Bonen meite Strecten mit Den charafteriftifichent (STeticherbilduntgen, Den gefrizten (Be=

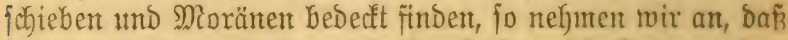
in einer früheren Beit, twelche Der ickigen bireft voranging,

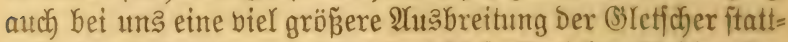

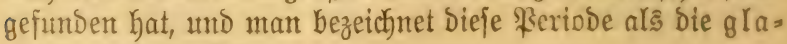
ziale oder bie ciszzeit.

\section{Tätigleit bes $\mathfrak{B i n d e s . ~}$}

Neben Dem Waffer unt (E)

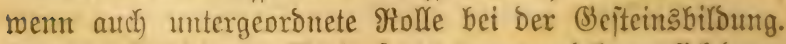
Der Staub wird bour Winde fortgetragent und Iagert ficty bant mieber an anderent Drten ab. J̄n Begention nun, two bie

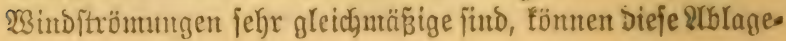


rungen, weld man $\Omega$ öp̃ nennt, eine ganz bedeutende Mäct)= tigfeit befommen; bejonders in China tourben foldbe bon

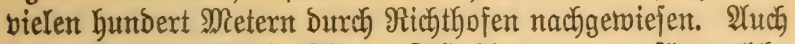

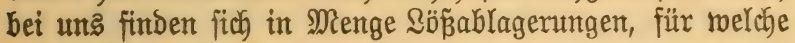
eine äolifjche Billoung anzunefmen ijt.

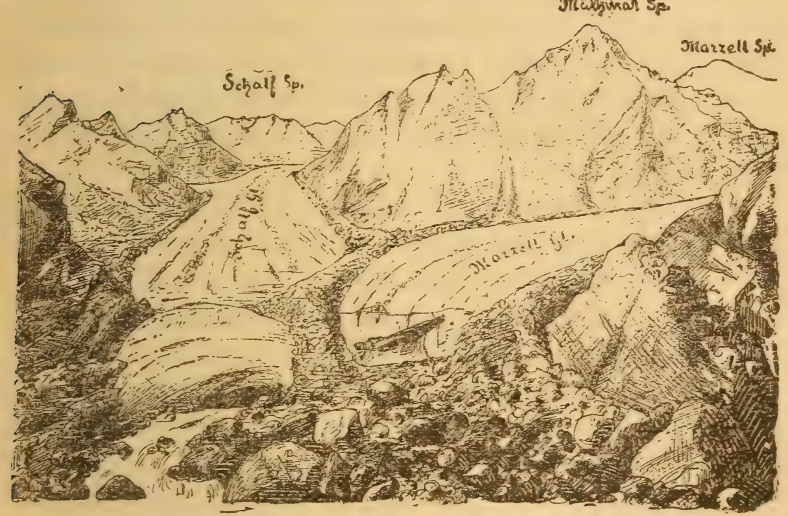

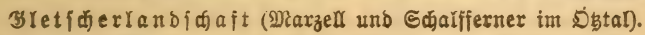

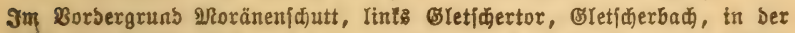

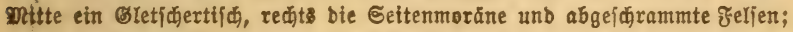
auf bem శ̊erner פitttelmorăne unb Seitenmorănen, im Şintergrumbe am Muthmal=\{togel grobe \&irnmulbe unb Settengletijher.

Wir baben noch einen febr midhtigen Faltor zł betrachten, ber bei ber Bildung ber Sebimentärgeiteine mitwinft, nämlich bie Tätigfeit bes organifchen \&ebenz.

\section{Tătigfeit Der Drganismen.}

Die oben nur furz ermähnte chemifche Tätigfeit Des Mafjers

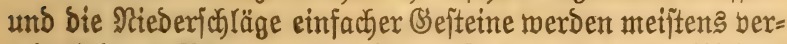
mittelt Durch SFlanzen ober Tiere, fo bor allem bie sieber: 


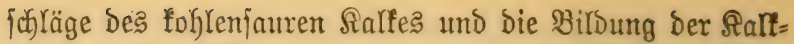
gejteine. Die \$Flanzen entziełen Dem im Wáfjer gelöiten

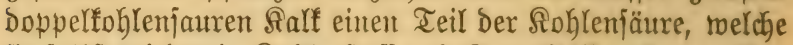
fie ferbjt roieder in Rohlenftoff und Saueritofi zerlegen. Ter

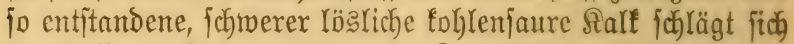
im Waffer nieber und bildet eine Salfablagerung, ber ङauts ftoff geft in bie @uft und das $2 a$ affer, wirft wieder orbdierend und gift fo Dem $\mathfrak{S}_{\text {affer neu }}$ Ifngriffipunfte zur Berftörung; der Sohlenitoff fchließ̌lich wird von Den Rffanzen aufgefpei= chert uns fann unter gïnftigen Bebingungen zur Roblenablage= rung fitfren.

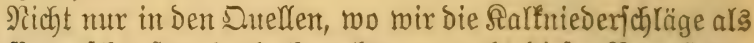

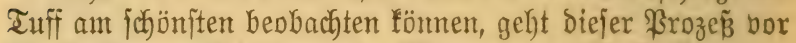
fitch, fondern er fpielt autch im Micere einte grof̉e Piolie.

Wägrend wir fo eine indirefte gejteinbildocnse fraft ber Pflanzen fennen gelent faben, finden wir aud atblagerungen, welche Direft Dutrch Rflautzen= und Tierantjäufungen gebilidet find. Szierher gefören bor alfem bie jadjon Seite 16 erwölnnten

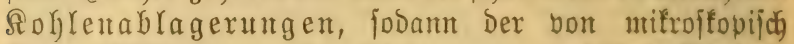

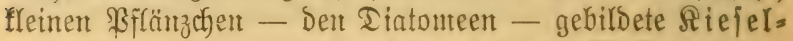
gur. Dieje $\mathfrak{B f l a n z e n ~ b i l d e n ~ j i u ̈ ) ~ n u t ~ D e r ~ i n t ~ W a f f e r ~ g e l o ̈ p t e n ~}$ Riejelfäure żierliche Sferette, weldhe zum Teil mur $1 / 300 \mathrm{~mm}$

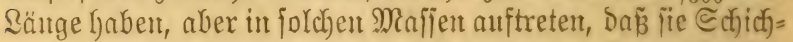
ten vou vielen Metern Meäd)tigfeit auffbauten. Terartige $\mathfrak{B} i=$ Dungen, an Denen fich in erfter Sinie Pflanzen beteiligen, nemt unan phytogene (s) efteine, ifnent werden die zooge =

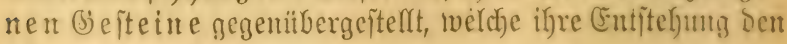
Tieren berbanfen.

Die Tiere twirfent in äfnthicher 23 cife twie bie Fflanzen, teils indireft durct) Den Berbrautch bon Eaueritofi unt bie bei ihrer Berwejung entiftefyenden Berbindungen, teils bireft burch

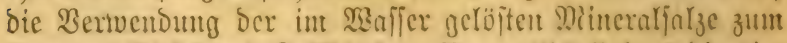
Baut bon Sicjel und Salffichalent. 2iamentlied) find cs bie nic= 
Deren, in Meere oft in unzäbligen Maffen Yebenden Tierchen,

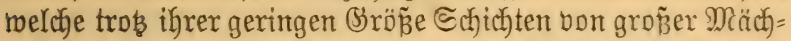
tigfeit aufbauten. So finden wir cinen Tcil Der frreide unt eine Menge anderer Sall= und Riefclgejtcine erfüllt bon Den zierlicbiten mifroffopijach Siebilden, melche meijt bon $\mathrm{Ur}=$ tierchen aus Den (Sejchlechtern Der Foraminiferen und Radiolarien ftammen; andere Echichten jeben fitch aus den Siejelnabeln Der Seejatwämme oder Spongien zıjammen. Die Tätigfeit Der ภorallentiere fömen mix heute noch in Meere beobachten; find Doch diefe fleinen Tierdyen imitande,

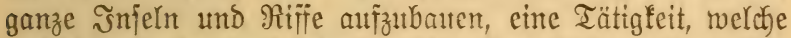
freilich mehr Den jest Yebenden fiorallen als Denen Der alten Erdperioden zufonmt. Inter den Strabltieren oder (F) in $D=$ bermen jind bie frindiben ober Seelilien zu ermähnen, welche in ben alten Formationen oft bie mächtigiten $\{a r l=$ ablagerungen exfüllen. YUch die હchalen ber $\mathfrak{M}$ u $j$ cheltiere

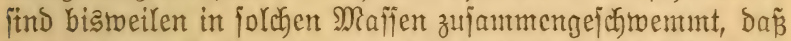
fie bas ganze Seftein zujanmenjezen. Je höber die Tiere ent=

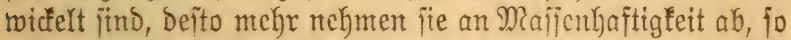

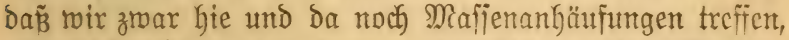
ofyne dicjen Tieren jedoch cinen eigentrichen gciteinbirbenden (e) Garafter zujchreiben zut fömnen.*)

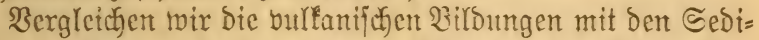

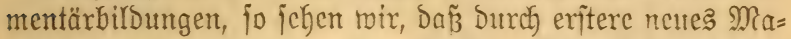
terial aut ber Tiefe Der (Frde nach Der Dberfläche gejchaffit wird, während bie Sedimentablagenungen weiter gar nichts baritellen, als eine fortwährende llmarbeitung und lofale Beränberung bes an ber Dberfläche fichon vorber borban= benen Materiales. Ter Bulfanismus roirt Dentnach in ber= tifaler, das $\mathfrak{W a f f e r ~ i n ~ G o r i z o n t a l e r ~ \Re i c h t u n t ~ a u f ~ u n j e r e ~ ( E r D = ~}$ oberfläct)e.

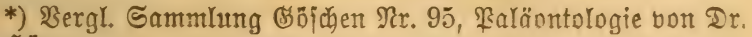

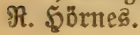




\section{III. $\mathfrak{A} \mathfrak{G}\{\mathfrak{d} \mathfrak{g}$ itt. \\ Die Bildung der Erdoberfläche.}

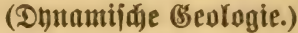

Nachbem wir das Material fennen gelernt haben, daş une jere Erotrujte zujammenjest, bleibt ung noch die 2Ufgabe übrig, zu unterjuchen, unter welchen Untitänden bie Bildung der (Erobberfläche mit ifyen Bergen und Tälern bor fitch ge= gangen ît. Die Sedimentärgeiteine find aus Niederfoblägen Des 2 afjers gebildet und Damu urjprüglich in Gorizontalen Schichten abgelagert, Demungeachtet feben mix fie aber zum gröpisten Teile in jofräger Stellung auftreten und miiffen bes=

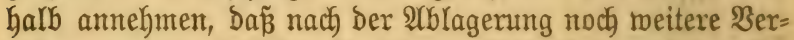
änderungen in örtlicter Seziebung (Dislolationen) bor fict) gegangen find. Bu Demiclben ßefultate führt un' die Beobach)= tung, Daß̃ wix auf Den höchjten Berggipfeln in ben Bejteinen Beriteinerungen finden, welche für eine Tieffeeablagerung iprechen; mo aljo jest ein hoher Berg fteht, muste früher tiefes Meer getwejen jein. Da mun faum Dentbar ijt, Dẫ frither auf Der Erde wejentlich mehr $\mathfrak{W a f f e r ~ b o r b a n d e n ~ w a r , ~ a l \$ ~ j e s t , ~}$ fo find berartige (5rjheinungen nur burch ein mit bedeuten= Den Nibeauberänberungen berbundenes Sch anten ber (5rdoberfläçe zu exflären.

\section{Sontraftion Der Crorinbe.}

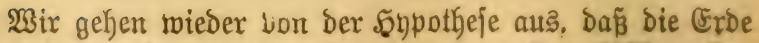
auts einem urfprüngliç, gasförmigen, Dann feuerfliiffitgen $3 u=$ ftand erjtarrt ijt. Mit Diejer Erjtarnung ging natitrlich auch eine Bufanmenzielyung, eine Berringerung Des నolumens Қand in ફand. Bon Dex Beit $a b$, Da fich um bie Eroe eine ftarre Srujte gebildet Gatte, traten in stefem Mantel ganz $a b=$ 
nornte Spannunģ̧⿻erbältnifīe auf, ba ber Mantel zwar bas Bejtreben hatte, fict Dem inneren Rerne anzulegen, Dex inmter mehr zufanmemjohrumpfte, aber Durch jeine Starrheit daran berhindert murbe. Schlię̧lich muñte es zu einem Brechen uno Berjobieben in Dem Mantel fonmen, um Die Wölbung zut ver= ringern; einzelne Teile fchoben fith Ḧbereinander, andere wurben quer geitellt, und badurch ergab fich für weitere Maffen \$lab, um abzufinfen. Tả Bild bes urfpriunglich gleichnäß̈igen Mantels tourbe mut ein jehr vermorrenes, bie

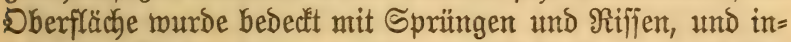
folge Der Berjobiebungen entítanden Einjenfungen und Čr=

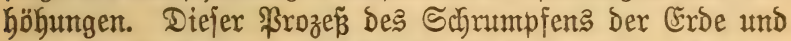
ifrer Srupte bauerte aber Durch alle Formationen bis zur Jestzeit fort, und jo jehen wir autch heute noch biejelben (Er= icheinungen mie dantals. Wir müffen unz nur babor hüten, uns bieje ミerjchiebungen mutweije und fatajtrophenartig za benten, fondem fie gehen fo langfam und gleichmä̈̈tg bor

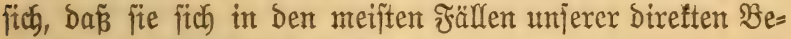
obachtung bolftändig entzieben.

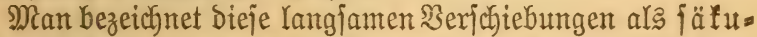
lare S2ebungen und Senfungen, ba es vieler Jahrbun= berte bedarf, bis ifre Spuren auffällig werben. Diejen $\mathfrak{B} e=$ wegungen unterliegen ganze アontinente ziemlich gleichmä̈̈ig, und dies exforwert natïrlich jehr bie Beobactung; Doch bleiben

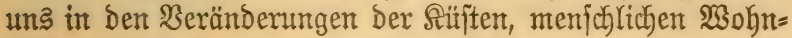
ftätten, Die jeß̧t unter Dem Meeresjpiegel liegen, Dent mert= roirroigen Bauten Der Sorallemifife, ferner edften Meereßbil= bungen hoch über bem jebsigen Meeresnibeau und anderen

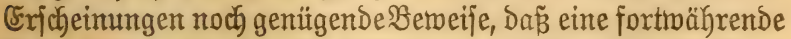

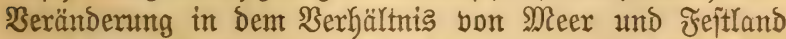

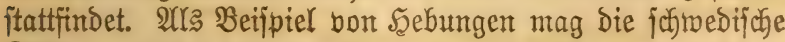
Ruifite angefübrt fein, too bie Szebung auf Şmunb bon einges

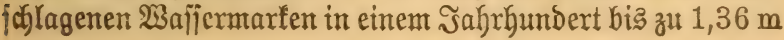


betragen Gat. 2H( Beifpiel fontinentaler Senfung bient am bejten $\mathfrak{S}_{0}$ lynejïn unit Der fontinentalen Tiertoelt, bie mir bort finden, und Den grozenen Rorallenbauten.*)

Welche reitgehenton $\mathfrak{B c r a ̈ n}$ derungen foldhe lontinentale Berjchiebungen mit fich bringen, lernen wir exjt fenuen, wenn twir bie geologifchen Perioden in Betradyt ziehen; ganze Weltteile, welche jebt Fejtland find, waren früfer überflutet, und in einzelnen Fällen fönnen wor noch auf das flarite bas Iangfante Wororingen Des Meeres itber das alte Feftland in geologifchen ßserioden nachmeifen (Transgrefition).

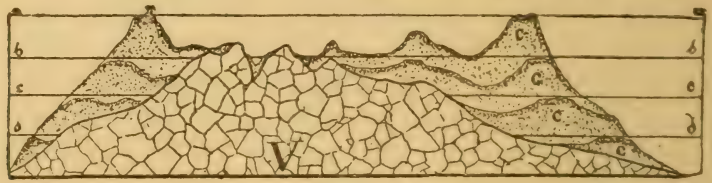

Rorarlentiff= 2 troung.

V Bulfanijher Berg im Sinfen begriffen. C Die Rorallenriffe um benfelben. $b-b, c-c, d-d$ Beridjtebene Meeresflädje unb die bamit berbunbenten Sta.

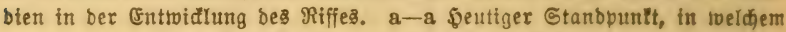
bas Rorallenriff nur noci eine 2rtoll bibet.

\section{Gbebirgabilbung.}

Wie bie gropenen Errbmaffen im ganzen, fo unterliegen auch

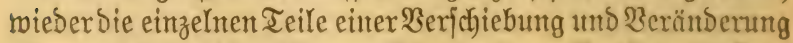
ifhrex

*) Die riffbildenden Sorallen leben nur in geringer Tiefe unter bem Meerespipiegel; num finden twir aber Riffe, bie bis zu be= beutenter Tiefe hinabreichen; Dieş ift nut baburch ertlärlich, baß ber Boben frilker nutr twenige Meter unter bem Meereşpiegel lag, auf bent fich Die Roralen anfiebclten. Durdy fortbauterno lange james Sinfen des Untergruntes twurben die Sorallen gezmungen, immer wieber auf den alten abgeftorbenen Stb̈fen aujzubanten, um nidht mit in bie Tiefe zu finfen. So entîtehen bie Soralfenriffe und Roralleninieln. 


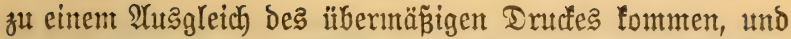
Dort werbent mu bie Maffen entrweber zufammengefoboben und emporgeljoben, oder auth es hat fich \$labs gebildet, unt ein

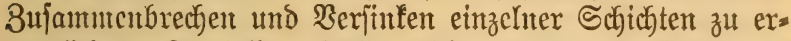

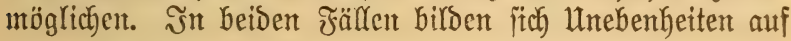

\section{A}

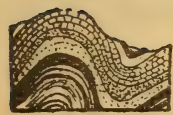

\section{B}

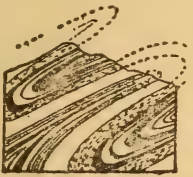

Berfuiedene テ̃altenbilonugen.

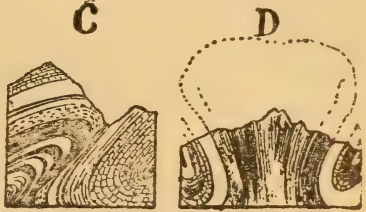

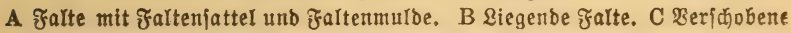
Falte. D fächerftellung bar ซalte.

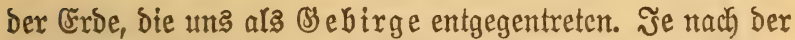
borrwiegenden Stmitur berjelben unterjicheiden wir Falten= gebirge oder Brutchgebirge. Die Faltengebirge, Deren jobjönftes Beifpiel unjere A(rpen*) bieten, treten uns immer als

A

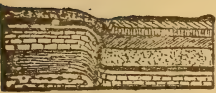

B

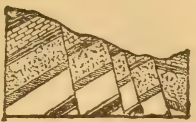

Bermerfungen.

C

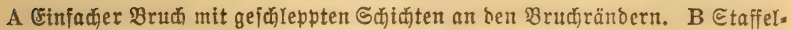
brudf. C Grabenberjentung mit foriten (H).

*) Die 2rrpen find in ifrem zentralen Teile megr oder minder in Fädherfalten geftellt, baran reift fich nach aufen ein Snjtem von fomplizierten liegenden Falten, weldhe in rubigere einfache

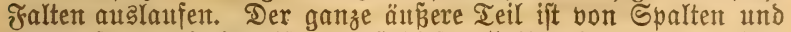

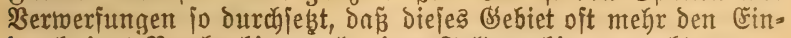

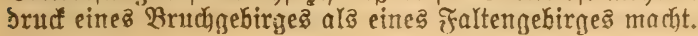


langgezogene Settengebirge entgegen; ifre Struťtur exinnert

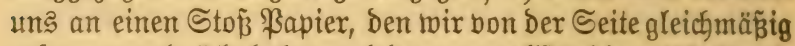

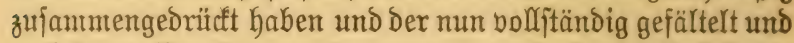
zerfnittert ift. J̃m groß̋en ganzen berlaufen natürliç) bie Falten rechttwinflig zur Dructrichtung, Doch fönnen auch) lofale Störungen in Mienge auftreten; bielfach) fommen bazu nod) Brüctje und Berwerfungent, wie fie leicht bei einem fo fpröben Material, wie bie Bejteine find, borfonmen, jo baß̄ ber IUfbau ein äuñerft berwiffelter und fomplizierter werben tann.

Die Schichtenfaltungen felbit ftellen balb einfacte $\mathfrak{A} u f=$ biegungen Dar, bald find jie boljitändig übereinandergeidyoben (Iiegende Falten), bald nit einer Bruchlinie berbunden uno verzerrt (gejchleppte Falten), ober wir finden auth bie Schich)= tent in Fächeritellumg aufgeriçtet.

Tie Faltung Der (3) ejteine geht bejonders bei Den Scjiefer= geiteinen bis in bie fleinjten Teile und wirb bann als ซ̛̈̆l= tełung bezeicjnet.

Un eine Derartige ₹altung bes fonft fo fpröben Materials

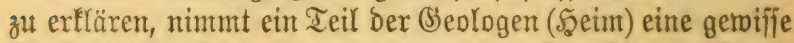
Blaftizität bes Miaterials unter enormem Drute an, währeno andere (Siümbel) eine feine Bertrïnmerung Des (3) iteines zu einer plaftif chen Mafije ber Faltenbildoung vorangeben lafijen.

Den Faltengebirgen ftelfen wir bie $\$$ ruch gebirg e gegen=

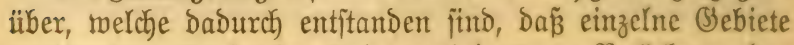
aneinander abgejunfen find, mobei es zu $\mathfrak{B}$ rïchen oder Bermerfungslinien fam. Das Stggleiten fann in groß̈en Tafeln geffyehen, wir fprechen bann bon Tafelbrü̈chen, ober ein treppenförmiges fein (Staffelbruch); bald ijt ber Bruct) mur auf einer Jlanfe crfolgt, bald fehen wir Das (s)ebiet an beiben Flanfen abgejunfen (ङ rabenverjenfung). Die

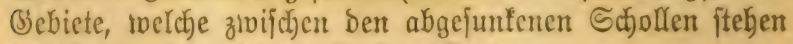

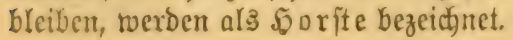


Bei bent Rapitel ïber Bebirgsbildung haben wir unt auth Der bulfanifichen Aräfte zu exinnent, weldye gleichfalls imitande find, hoke B̧erge emporzumerfen. Wir haben fdyon ermähnt,

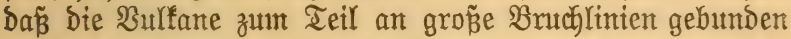
find; aber fie billen nie die Ilxjactje einer Berjprengung bes

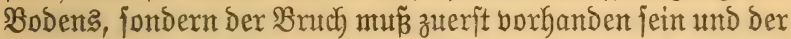
Eruptionsmafje Gelegenteit zum cimporbringen geber. 2)t dieje $\mathfrak{B e i j e}$ entiteben bie vulfanijchen (Sebirge.

\section{Erobeben.}

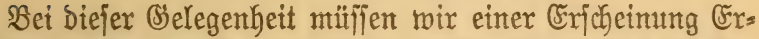

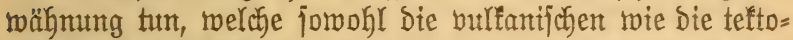

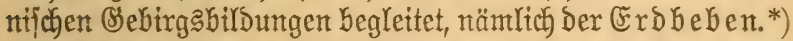
Die Erobeben befteben in Schmantungen und Stöpen beכ Erobodens, welche von einem Bentrum autsgehen und fitc bon

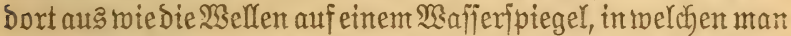
einen Stein getworfen hat, fortpflanzen. Die (Erobeben merben

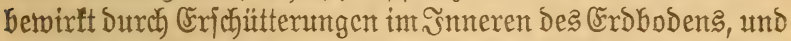

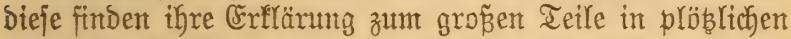

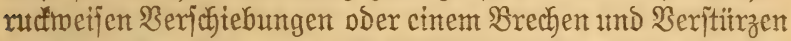
ber Schichten. ङE exfolgt aljo fier infolge allzu groz̃er Span= mung Der Borgang fefr plöblich, rolcher fonit mux fefr Iangiant und inmerfbar bor fich geht. Man bezeidnet bieje

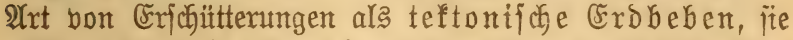
zeidynen fict) in Der Regel Durd lange Dauter und weite $\mathfrak{B e r}=$ breitung aus.

Andererjeits bewirfen natïrlich auch bie andringenden Dampf = und Feuernaffen ber Bulfante, welche bentüht finto, die auf ifnen laftende Decfe zu Durchbrechen, unter Itmftänden

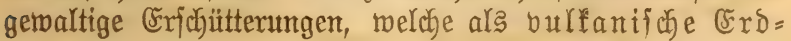

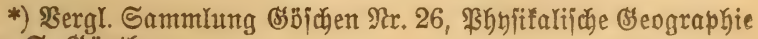
bon S. Bünther. 
beben zu bezeichnen find. Sie beforänfen fidc) nur auf bie Untgegend Der tätigen Bulfaute, und das Crobebenzentmum

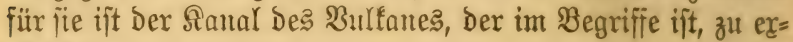
plobieren.

Rleinere lofale Erobeben, fog. Einfturzbeben, entiteben zHmeilen infolge von Interhöhlung Deక Boben Durch Waffex und श्रachitürzen Der Darïber liegenden (Siejteine. Sie futo jeboch bon feiner meiteren Bedeutung und mögen mur Der Boll= itänotgleit halber Crtwähnung finden.

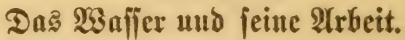

Dent ummterbrochenen Sreislauf auf umferex Crob unter= liegt, twie wir fachon bei Der Bilbung Der Sebintente gejefen

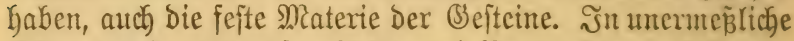

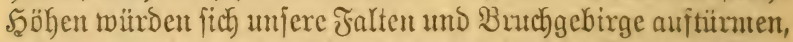
wenn nicht eine zerjtörcube und auggleichende fraft unit ifnen nałezu gleichen Schritt halten mürbe; e⿱

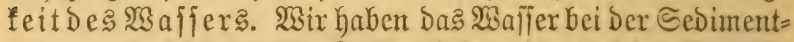
billoung als fob̈upferijche Siraft gejehen, hier bei Der Betract)=

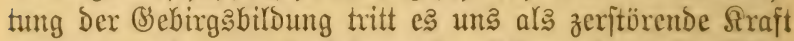

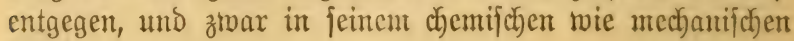
Wirfen.

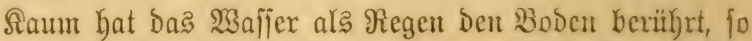
beginnt aud fichon bie Berftörung und bas Beptreben, wieber

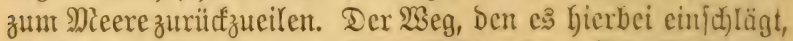

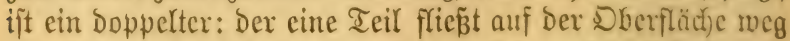
und fithrt babei alle Soinderniffe mit fich, Ivelde fict) il)u in ben $\mathbb{S e g}$ iteflen, feine Tätigleit ijt aljo cine med) a ein anderer Icil Dringt in bie Tiefe ein und wirft Dort Durch

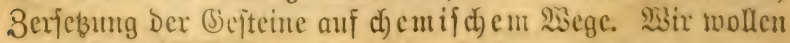
zuntächjt bicjen \$rozç etwą cingel)entex verfolgen.

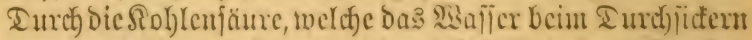

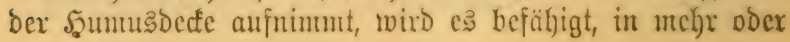




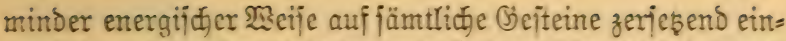
zuroirfen. Cinzelne (j)ejteine, mie falf, Dolonit, Gips und

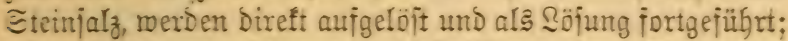
mir haben jofon gejehen, wie bieje Mineralien fpüter, ment

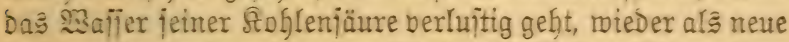
Eedimente afgelagert merden (Eeite 38). Andere Dineralien, mie Die @ifiłate und maljerfreien Minerafien, müjen erit in Ib̈slifje maljerbaltige Mineralien umgemandelt merden. Eo mirb Der Plnhyorit in Gips, Die meijten Eilifate in Ton um= gemandelt und Dann gelojt: ebenjo bervirft Der Eaueritoï in Majler eine Drybation, Die Sohlenjäure eine llmwand lung in Iöslidge fohlenjaure Berbindungen ober Rarbonate. F 5 mürbe zu meif fübren, auj Dieje oft jehr fomplizierten $20 x g a ̈ n g e$ näber einzugeben; betráden mir Deshalb pofort Das Fnorejul=

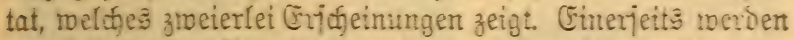
Die Gejtcine Der Dberflähe zerjebst und in leifht Iostiche um= gemundelt, mas man als 2 erroitterung bezeiffnet. Vie

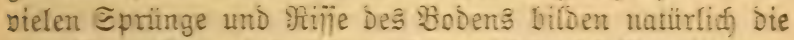
Angrifïpunfte, und jo jeben roir bon Diejen Die \$ermitterung auggehen und um jich greifen. Andcrerjeits rerben bie ein= jiffemben : und fonmen num als Mineralquellen mieber zu Tage tweten.

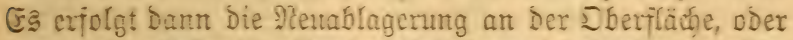
aber in Den tiejen Epalten ber Geiteine und fübrt Dort zu Bil= Dungen bon Mineralgängen in ber Tieje. Tout finden wir Die in ben 3ebengeiteinen utifrojfopiji fein enthaltenen Frze in grö̈eren Miengen zujammengetragen, io baв ï̈f ein

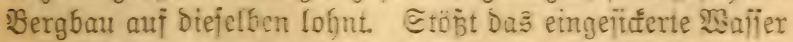
in Der Tieje auf leidht löslidge Geiteine, jo toüjoft es Dieje auts

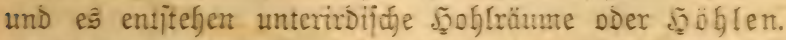

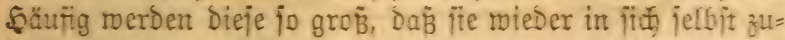
jammenjtïrzen und jo zu Den jofon erroafyten Finjturzbeben füfren. 


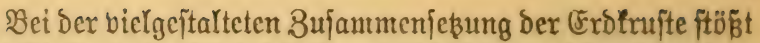
baక $\mathfrak{3 a f f e r ~ i n ~ D e r ~ T i e f e ~ h a ̈ u f i g ~ a u f ~ S c h i c h t e n , ~ w e l d j e ~ e s ~ m e n i g e r ~}$ reicht Durchjitcern laffen alङ andere, und es fieht fich bann gezmungen, auf Diejer Schicht hinzufließ̃en. Streicht Die un=

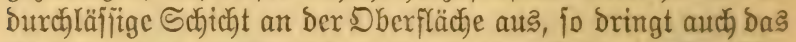
Waffer auf einem Spart heraus und es entiteht eine Duelle.

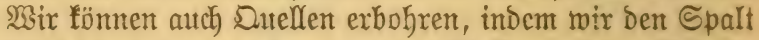
fïntrich fchaffen, in weldyem Dann Daక Waffer nach Dem ßrinzip

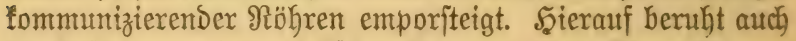
die (Exfchcimung Der artefiticten Brumnen (fieke Ftgur B).

\section{A}
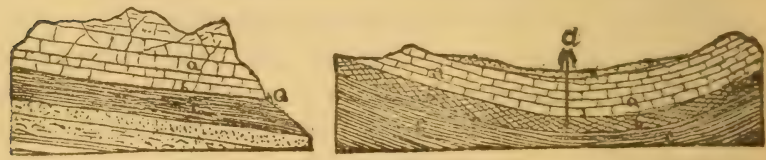

Quelrbiloungen.

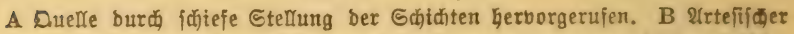

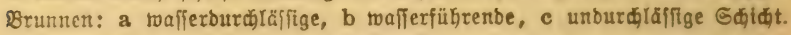
Q Quelle.

Tie mechanifdye Tätigleit bes 2Saffers fomobr in feiner

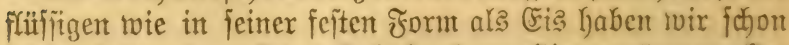
frither beiprochen. Sie bejteht in cinem Sombegrämmen aller loderen Seindernifie, welche fich feinent Saufe in Den $\mathfrak{W e g}$ ftellen, und man bezcidynct Diefe Tätigfeit als (Fo r fi i n. Qutc, Gier Dienen als ?Yngriffझpunfte zuerit bie zah)lfojen Eprïnge und Rivije Des (Siciteins, weld)e immer twieder bergrïbert und erneut twerden, fo Daß̧ Daburch cine fortmährente Soctentug

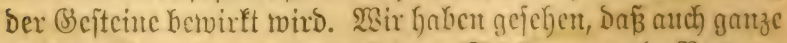
Schid)tentomplere bon mähtigen Sprïngen umb ミertver: fungen burchfeßst find, und es ift natïrlich, Dafi bicje Dem Wafier tvillfommene Ûngrif̈spunlte bicten. Etöß̈t aber ein 


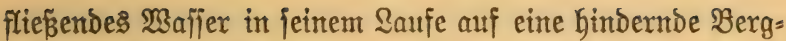
fette, welche es nid)t umgehen fann, fo ftaut es fich anfangs zum See auf, bis es ifym gelungen tift, fich fo tief einzunagen,

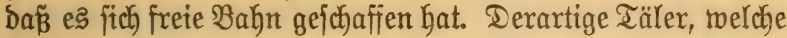

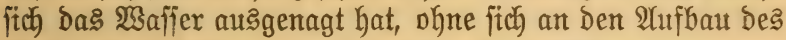
Sebirges zu Galten, nennt man Erofionstäler.

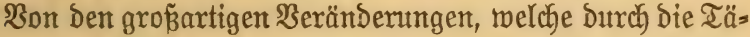

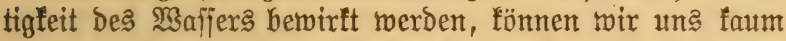

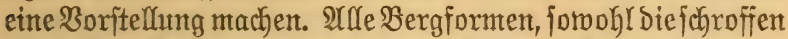

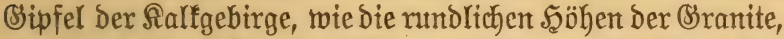
ebenjo twie die Schluchten, Täler und Escnen, find durch das Waffer geformt umb gebildot. S̈ebirge, Deren Şöhe unjern Göchfiten (Sebirgen glcichtam, fint bis zur flact)en Szügelland= ichaft, ia bis zux Ebente abgetragen, und mux Die gefalteten und aufgerichteten Schichten zeitgen noch bon ben fritheren Störungen, welche Dort ftattgefunden Gaben. Der ganze über $20000 \mathrm{~m}$ mächtige Schichtentomplex ber Sedimentärforma= tionen roar bom Waffer trantaportiert und zum $2 \mathfrak{A}$ g gleich der Göhenunterifjiede bertwendet toorden.

IV. $\mathfrak{A} \mathfrak{b}\{\mathfrak{d}$ ) nitt.

\section{Бilftorilfche Geologie oder formationslebre.}

Während wix uns in Den borangebentoen 2Ubjeynitten einen

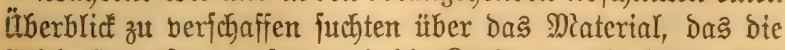
Frofmite zujammenjebt, und die Aräfte, melche babei tätig maren, ftellt fich die hiftorifche (Şcologie Die Unter\{uch) ung ber einzelnen Schichten ober Formationen und, mit Gilfe der Darin enthaltenen l̈berrejte, die Entrid irdifchen Betrobnex zur UAfgabe. 
Wie mir gefefen faben, fitellen die bielen (Slieder ber Sebi. mentärformationen mrr eine umunterbrochene lturwanolung

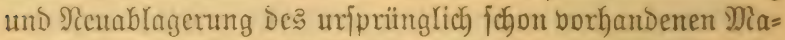

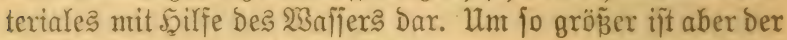
zeitliche Unterfchics Diefer Âtblagenung, welche, twie beute,

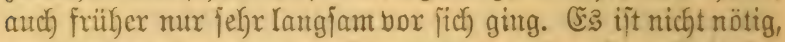

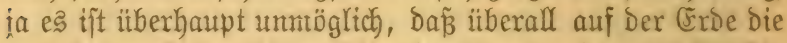

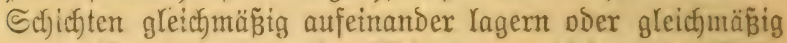
ausgebild fet find, Denn Der SYblagenung auf ber einen Seite ftand ja immer cinte Berftönutg auf Der anbern Seite gegen=

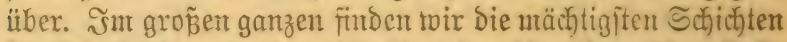
burct) Das Mleer abgelagert, mährend auf Dem Damaligen reeit= lande feine ober mur geringe 2fblagenungen bor fich gingen, fa im (Segenteil bon Dicfen Rande umuterbrocfen abgemajonen uns weggeidfwentunt rumbe. (5) fonnten alfo in einer

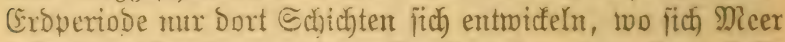
befand, mäfrend an antern \$unften, Dem Damaligen ₹rejt= lande, feine DDer nur twentg gleichalterige Bciteine zum 21 bjaß

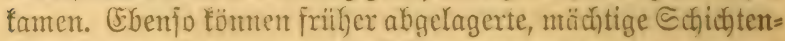
fomplexe in fpäterer 3cit wicoer volfftänoig ober bis auf wenige litberrejte abgetwafden werben und berloren gehen. Ia $a$ mir trobzocm fajt überall Meereşablagerungen finden,

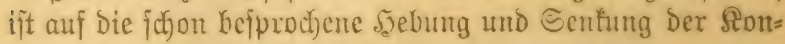

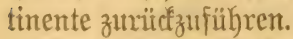

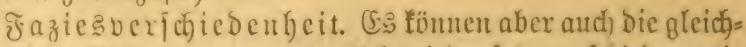

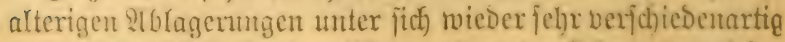

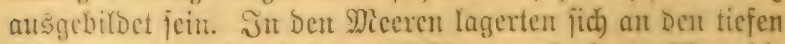
Etelfen unr Sialf unb feiner Edflanum $a b$, in weld)ent bie

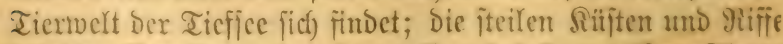
belebten fiorallen und auf Dem (Srumb feitgemaed)fene Tiere: in Den Strünungen Der Mieere munde unchr Sand unt

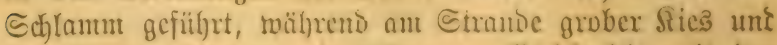
(Berb̈lle den llntergrumb bildeten. (j)leid)seitig utit ber 
martnen 2 blagemungen lömen aber auct auf bem Feftlande terreftriface Bildungen bor fich geben; fumpfige Urmälder merden unక als f̊bhlenablagenung wieber entgegentreten, Die Ströme merfen Schotter auf, in Den Binnenfeen lagert fitch Schlamm mit ben Berwofnern jüpen Waffers ab. Altīerdem fömen auch noch die berichiedenartigiten bulfanifchen 2rus=

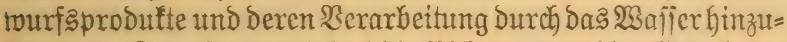
fonmen. Surz, jo mannigfach die Bildungen auf Der (FrDe beute noch find, fo mannigfach baben wix fie uns antch in fruberen Groperioden borzutellen. Man bezetchnet Dicfe forwohl in ifrem (Befteinscharafter wie in Den erbaltenen überreften fich hungebende Berfojtebenlyeit einer gleidgalterigen Formation als ₹azies und ipricht Dentnach von mariner, riefjee=, rito= raler, terrejtrijatier ujw. Fazies.

Begriff Der Format i on. Durch jorgfältiges Bergleicyen

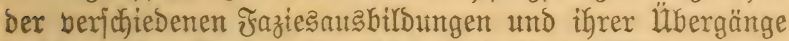
fucht mun Der (Seologe fümtliche gleichalterige 2lblagenungen zujammenzuitellen und bezeichnet fie als eine Formation.

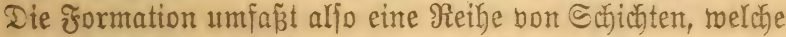
unter fich jehr berichiedenartig ausgebildet jein fönnen, abex Doch ein gleiches arler befizen; ite ijt Dantit zugleich ein zeit= licher Begriff und fällt zufammen ntit einem geminjen Stadium Dex Entrotcflung Der Eroe und ifrer Betwolyner, einer $[0=$ genanten geologifach (Eroperiode.

Urtersbeitimmung ber Formation. In num das arter einer zu unterfuchenden Schicht zu beftimmen, twirb zuerit die Stellung derjelben im ganzen Sebirgsfyitem exforjaft. Man unterjucht, ob bie fragliche Schicft nicht von anderen uns befannten Schichten überlagert ober interlagert wird; Dann mird Dex (Sejteins charafter in Betracht gezogen; vor allem aber ift zu unterfuchen, weldhe 2 erjteinerungen un

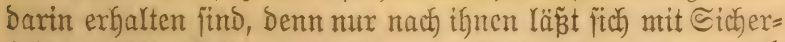
Geit Das arter beftimmen. Denmady fällt auth Die Sautptauf= 
gabe der Giitorif fte in erungen, ifres gcologifchen 2 luftretents und ifrer (ents micflung, ein ভtudium, das als felbitändige Wiffenjichaft Saläontologie - bie Bermittlung von Beologie uno $800=$ Iogie birset*).

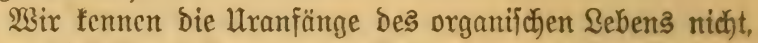
benn biefelken fallen in eine (Eroperiobe, aus weldter uns feine erfeutbaren Spuren megr erbalten find. Tabei ntïffer wir vor allem Daran denfen, Daß̃ unక nur bie Şartgebil'be Der Tiere uno in feltenen Fällen die \$flanzenftruftur erbalten ift:

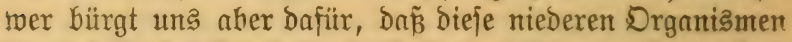

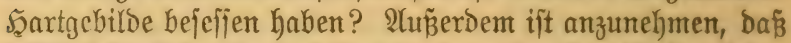
felbit fehr feite Şartgebirde in Dem Durchgreifenden Utm= wandhungsprozeffe, weld)en wir zur Entiftehung ber friftal= linifchen Schiefer angenonumen haben, gleichfalls mit un= getwambelt tworben find und fict Darum unferer Beobactumg entziełen. In Den ürteiten Schichten, aus Denen uns Ber= iteinenutgen befant find, treten uns. Deshalb figon ber= hältnismäß̈ig hoch entriffelte Tiere entgegen; berfolgen mix bie geologif(t)en ßerioben meiter, fo fehen wir in großsen

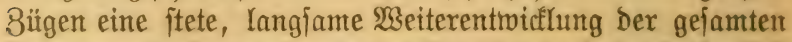
\$flanzen = unt Tiertwelt unt eine Afmäherung ber urfpriinglich

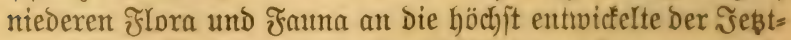
zeit. Dies gilt aber nur bon bem Billo im gropen ganzen, in einzelnen (Bejchlechtern fällt bie höchjte Formententmidlung in längit nergangene Eroperioben; fte fterben mieder aus oder

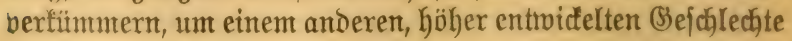
Blaßs zu mactjen.

() Iieber ung Der Formationen. Um eine flare überifdett zu befommen, Denft man fich) alle umb befannten ๔(d)

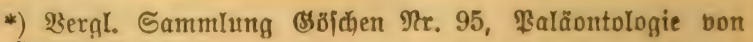

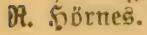


einandergelegt und gliebert fie in größzere (Simppen, welche Den Eintritt einer neuen (Epodye in Der Entwicflung Der Bflanzen = und Tiermelt bezcidynen. Dieje (3mupen ober Beitalter zcrlegt man fobam wicber in Formationen, die eine in fich mefr oder minder abgejuloliene \$extode ber Erogefdichte Daritellen und gleichfalls Durch Durthgreifende Merfmale der Pfflanzen= und Tierwelt dharafterijtert find.

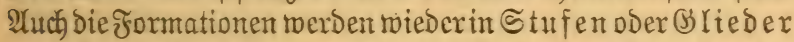
zerlegt, eine (Finteilung, wcldye fich Durch Das âftreten be=

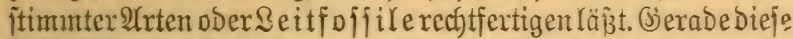
¿eitfoifitle, weldye in furzen beftimmten ßerioden über grop̈e Strecfen berbreitet borfommen und fith baher auf eine einzige

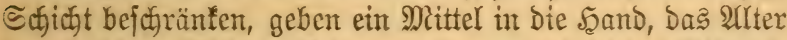
Der einzelnen Schicten ficher zu erfentmen und bieje jelbit noch eingebenter in 50 orizonte zu gliebern.

Bei ber mun folgenden fuzen Bufammenjtellung Der For= mationen muź ich mich natïrlich Darauf bejaränfen, ein mög= lichjt gedrängtes Bill Der Fommation int ganzen zu geben, ofne babei auf cinzelheiten ober auf Scitfofjille für beitimunte \$orizonte eingchen zu fömten.

\section{Erites Zeitalter der Erde oder die archäilchen formationen.}

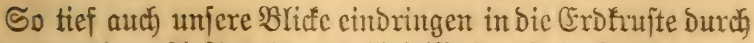

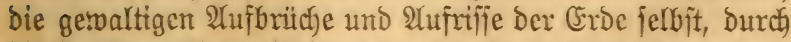
bie Crofitonatäler und tiefen Bergmerte, fo fömten roir Doch nicht bie ältejte Formation feitịtclllen, welche gebild burch bie urfprüngliche Eritarrungatrufte ber crobe. Was wir fenner, ftnd alles fchon burch Das Waffer berwegte und neu abgclagerte Maficn. Sir miifijen aber amefymen, 
Dẩ Die erite Eritamungsmafje alle die Subitanzen entfalten hat, welche roir ipäter roieder berarbeitet finden, und dürfen

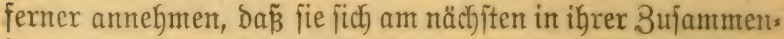

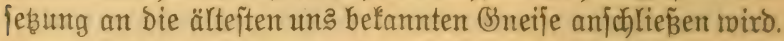

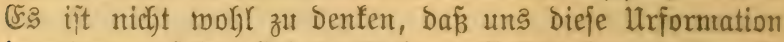
irgendino auf Dex Croboberfläctie zu Gefictyt fommt, da alle

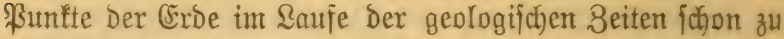
vielfachen Umtwälzungen und IMmänderungen unterlegen find.

Die ältejten formationen, welche unjerer Beobachtung zu= gänglich find, weichen bon ben fpäteren injofern ganz be beutend ab, als fie friftallinifocter Natur find, aljo nicht ein= fache, fonbern gemengte Beiteine Darftellen. Man bezeichnet fie daher auch als Iriftalrinifone Sdyiefergefteine. Über

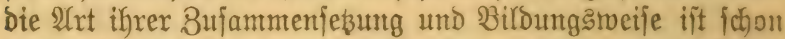
fritber (Seite 20 und 34) gejprod)en toprben, uno es bleibt noch) übrig, ifre gegenjeitige Sagerung und ihre geologifiche Stellumg fenten zu lemen.

Ias älteite (Slied ift die (S) neisformation, meldye in ber enormen Mächtigfeit bis zu $30000 \mathrm{~m}$ unter allen be= fannten Jormationen liegt. Im allgemeinen bemahrt fie cinten monotonen Sfarafter; Ditnnflaferige, fajuppige oder fein= ichieferige $\mathfrak{B a r i e t a ̈ t e n ~ m e c h f e l l a g e r n ~ m i t ~ g r o b f o ̈ m i g e n ~ o b e r ~}$ bichten, focheinbar ungejojichteten Maffen. In bent üäditigiten, genau ftudierten (Sneisłomplexe Des Bayrifden Maldes ither: miegt in Den unteren Bonent bie graue, itt Den oberen bie röt=

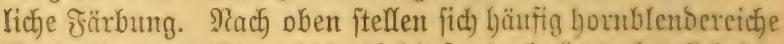
Barietüten cin (2tuphibolfatefer), bald mehrt fict) Der

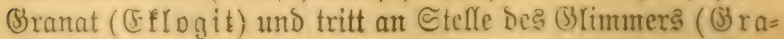

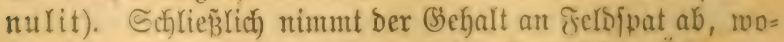
gegen Der (5) fimmer fich ntel)rt, und in allmäb)lichen lubergang fonmen wix zur zineiten (3) ruppe,

Der (S) rintuerfobefer= Formation. P(ud in diejer find wieder eine großze Dicife von \$arietäten zu berzeidunen, 
je nachbem ber eine ober andere Beitandteil borwiegend wirb. (Siehe Tabelle Seite 20.)

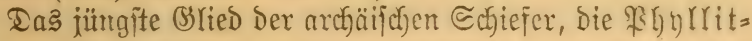
Formation, trägt zmar in Den unteren నagen nocf gantz Den

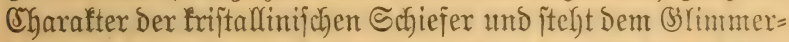
foriefer jebr nabe; in ben höheren Sgorizonten jedoch iterren fith immer mehr tonige Beimengungen eit, fo Dáz bicje (je $=$ fteine oft faum mebr nach ifrem (Sefteinscharafter bon Den Darauf folgenden jüngcren Tonjodidten zut trennen find. Man bezeidynet baher bie \$gyndite auch als IIrtonjatiefer oder übergangaggebirge.

$$
\text { G. Ossa }
$$

G. $a_{2}$ ber

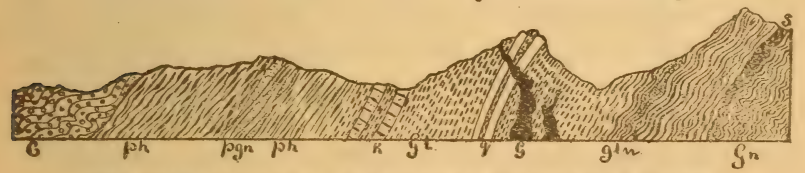

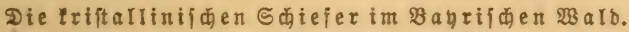

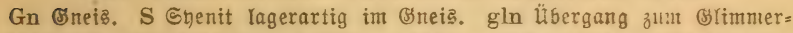

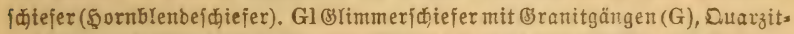

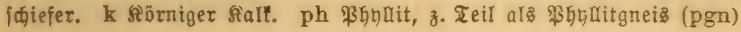
entruifelt. C ßambrium.

Bergebens f chauen wir uns in den friftallinif chen Schiefern

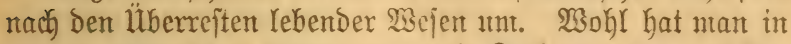
Den eigentiumlichen Sexpentin= und Salfgemengen aus Der Sineisformation die Ïberrefte eines grofien IIrfdyleimtieres zut erfennen geglaubt und e $50 z$ o on genannt, aber neuere Interfuchungen haben es nit Sicherbeit als eine unorganij che Mineralbildung erfennen lafjen. Es bleibt unts bennaw bie

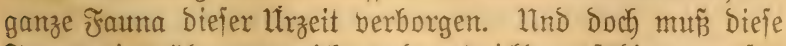

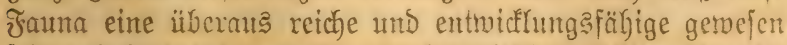
jein und batte jich autch bis zum (Fintritt Der nächiten \$3eriobe

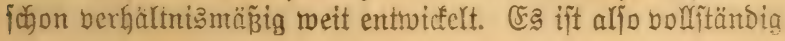




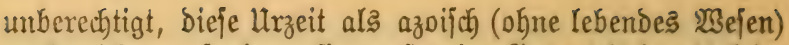
zu bezeichuen, fondern fie muß̈te im Begenteil ben Urfeim alles organifacen Sebens entbalten.

Tabelfarifde überjicht ber archäijchen formationen.

\begin{tabular}{|c|c|c|c|c|}
\hline $\begin{array}{l}\text { (Sejteits? } \\
\text { charafter }\end{array}$ & $\mid \begin{array}{c}\text { B)lie- } \\
\text { Derung }\end{array}$ & 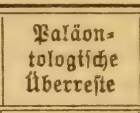 & \begin{tabular}{|c|} 
SIleidts \\
altrige \\
Eruptip. \\
geffeine
\end{tabular} & $\begin{array}{l}\text { Berbreitungş- } \\
\text { gebiete }\end{array}$ \\
\hline $\begin{array}{l}\text { Sriftallis } \\
\text { nildye } \\
\text { Schiefer- } \\
\text { gefteine }\end{array}$ & 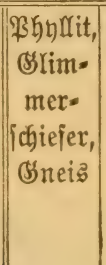 & unbefannt & $\begin{array}{c}\text { Diorit } \\
\text { Diabas } \\
\text { Syenit } \\
\text { Sranit }\end{array}$ & 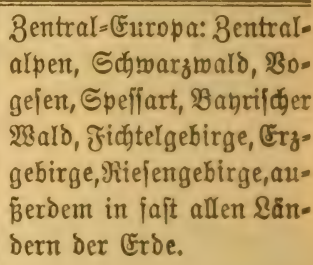 \\
\hline
\end{tabular}

\section{Zweites Zeitalter}

\section{oder die paläozoílehen formationen.}

Ier übergang von ben friftallinifdyen Schiefern in bie pa=

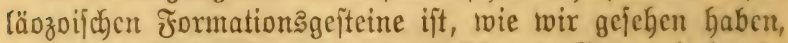
ein ganz alfmäh)(icher und eine beitimute Brrenze baber in

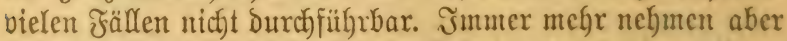

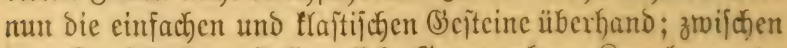
ocn Tonfchicfern itelfen jich (Srambaten, Ronglomerate, Santofteine und zum Teil felgr mähtige Sialfablagerungen ein. Ias roidytigite Merfunal aber jüno bic Berītenterungen, melche

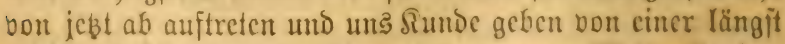

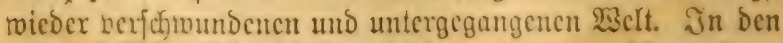

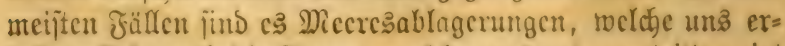
Galten jind, und bie ₹rauna, weld)e uns entgegentritt, zeigt 


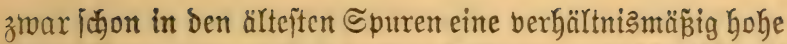
Entroiflung, aber ea find bod) noch Formen, weld)e fict) mit den cbet leberiden gar nidft oder mux febr jofrwer in Finflang bringen lafjer.

Es jind nur menige (sicjoblecter bertreten, aber dieje mit eincm ftaunenswerten Reichtum Der Formen und bor allem ber Sndiviouen; eine cinzige ๔pezies tritt oft jo maffentaft

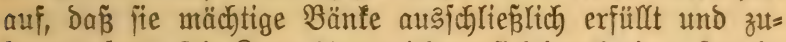
jammenjest. Die \& oralYen zeichnen fich burch einen fremo= artigen Sharalter aus und nähern jich exít allmäblich unjeren jest Yebenden Formen; ntan ftellt fie als Zoantharia rugosa und tabulata den jest Yebenden Hexacoralla gegenïber. Bon ben Strahltieren und (schinodermen jind es die jest fehr jel= tenen Seelilien ober frrmoiben, welute in biejem Beitalter ifren böchiten Formenreichtum entfalten, während die see=

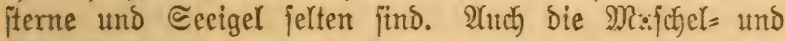
Sdynedentiere haben Durchgehend ein frembartiges SYlusfehen;

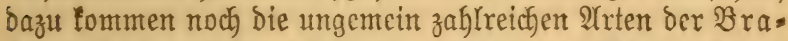
wiopoden und ber falentragenden Tintenfijabe aus dem

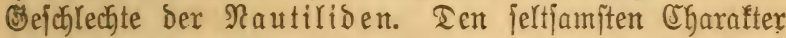
tragen unter den paläozoififen (Sliedertierent die Trilobiten, roldye bereits in ben tiefiten Sgorizonten in zahrojer Menge auftreten, um bann in ben jüngeren Schichten mieber boll= itändig zu berjofroinden; fie jind faum mit irgend eintem $\Upsilon_{e}=$ benden (Sejchlecht ber frebje in Finflang zu britgen. Csigent= tiimliche Fijche vout oft abentenerlichen (Se)talten beginnen in Den mittleren Sagen Diejer. Formationen, und Dazu fomment

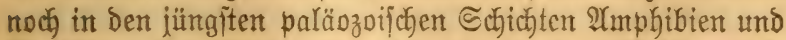

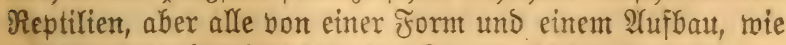
wir ifn unter ben jest rebenden Tieren nicht mebr finden.

Was die Bffanzentwelt anbelangt, jo fömnen roir fite erit von Der Beit an richtig beurtcilen, aus meldyer uns reichliche ter=

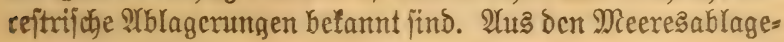


xungen find uns nur ÂTgen befannt, in Den Etcinfoblen= ablagenumgen Dagegen tritt umb eire flora entgegen, weld)e, wie bie Tientwelt, bon ber jeksigen bolffonmen berfhieben ift. Bor allem fint es bie (3) efäpifryptogamen, welche vor= twiegen uno zu riejigen Bäumen Kerantwachfen; Dancben treten noch einzelne Soniferen auf, mähreno bie unjere ieşige テ̃lora beherrichenden Difotyledonten noch fehlen.

So finton wir in Dem paläozoif hen Beitalter eine Mrtwelt entwicfelt, weldye uns in jeder einzelnen Form wie in ilgrem

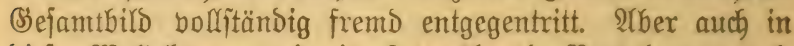
Diejer $\mathfrak{W e l t}$ fönmen mir eine fortmährende ßeräntoerung und Weiterentwidflung beobactenten, und eben auf Dieje Berände= nungen, auf bas 24usfterben einzelner (S)ejchlect)ter und bas 2Yuftreten netter grüntoet fict) Die (Sliedenung in einzelne fror= mationen.

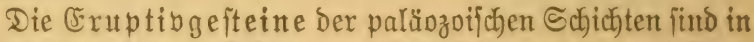
ben beiden älteren (S)liebern noch biefelben ober mentigitens

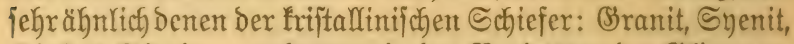
Diabas, Tiorit - und zmar in Den Barietäten Der Brimmer= biorite - walten fier vor, während bie jüngeren (S) i ieber burch bie mafienthaften Eruptionen bon Melaphyr und bejonders Dutarzporphyr ausgezeidynet find.

\section{Sambriun unb Silurformation.}

Parx an twenigen Stellen in Tentid)land, an Norbrande

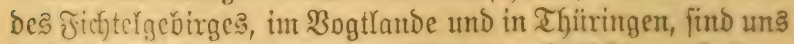
bicfe ältciten Beritcinenutgen fübrenten @efjicten erbalten;

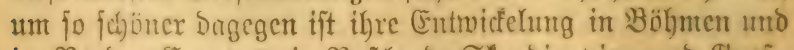

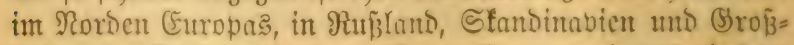
Gritanten, fotvie in Rordamerifa, two fie ungemein auşgerchnte ¿änbergebiete zufummenjetzen. Iie (Sicjamtmäct)tigfeit biejer Formation fant iiber 20000 Mieter erreichen. 
Der (B) fiteinscharafter, Der fier bortuiegt, ift ein toni= ger und fandiger, zu welchem fich untergeoronet auch Salt= ablagemungen gejellen. (Setoöhnlich ift Der übergang auts Den Urtonjchiefern Dex \$byllititufe ein faum merflicher, jo baf in ben unteren Fonizonten Tonjfitefer bon fatwarzer und grauter Farbe vortwiegen, welche nicht felten als I act $=$ und (Sriffel= jobiefer Dermendung finden. Brijchen Diejen Tonjatiefern fitellen f̈̈ch Sirantmacfen und Sanditeine ein, welche bejonders in Den Göberen Sorizonten an Mächtigteitzmehmen. Sdytwarze

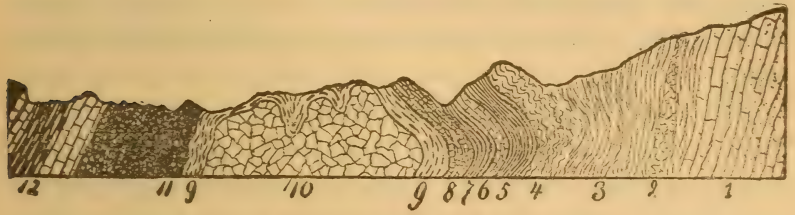

Silur und Devonim fictergebirge.

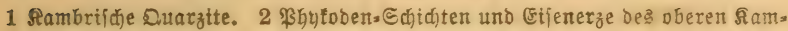
brium. 3-5 unter=Silur, quarzitidje Єđiefer uno untere (6raptolithenfifiefer. 6-8 Dber=Stlur: 6 Ralfe ber Cardiola interrupta, 7 Braptoltthenichiefer, 8 Ientalutten=ふalle. 9-11 Devon: 9 unterbebon. Rereitenidiejer, 10 Diabae, 11 Diabastuffe. 12 Dberbebon mit (Sontatitenfalfen, Sd)alfteinen uno RIt)mentenf(jidten.

Ralfe treten nux bereinzelt auf, find aber Dann faft immter

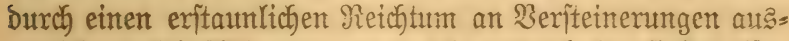
gezeidntet. Die 2rblagenungen, welide uns erbalten find, weifen alle auf Meeresbildungen Gin, Daher getiören autch alle $\mathfrak{V e r}=$ fteinerntngen Den Seetieren und marinen Fflanzen an; mur zufällig find hie und da Betwolyner des ¿andes in bas Meer Gineingefpillt und uns erbalten geflieben.

Die prälambrifche und fambrifde Formation birdet

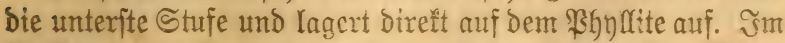
\$rälambrium jind es mur undeutliche હpuren bon Argen

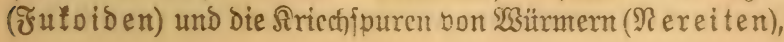


welche uns in ben tieferen Faorizonten Beugnis einer fófon vorhandenen Tier=und Bflanzentwelt geben. Tagegen beobact)= ten wir im Rambrium $2(b l a g e r u n g e n$ mit woblerhaltenen $B e r=$ iteinerungen, weldhe an einzelnten Drten boffitündig bas (B)e ítein erfüffen. Ien grö̈̈ten Teil ber Fauna bilden Trilo= biten, welche bon ben fleinen, faum $2 \mathrm{~mm}$ groß̉en $\mathfrak{A} r t e n$

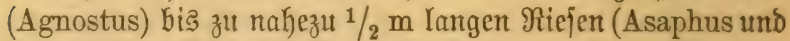
Paradoxites, Taf. III, F̛ig. 10) einen ungeahnten formen= reicftum erfemen lailen. Tie anderen Tiergruppen, bon Denen mur einzelne Bractiopoden (Lingula, Obolus, Orthis) benterfenstwert find, treten neben den Trilobiten in den Şinter= grutno.

Uuf Den fambrifchen Schichten lagert das unterjilur. 2fuch in biefen 2 Kblagerungen fpielen noch die Trilobiten

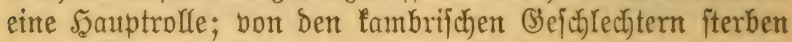
zmar einzelne aus, aber an beren @telle treten beinahe eben=

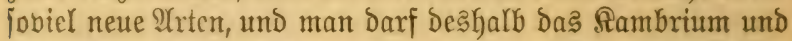

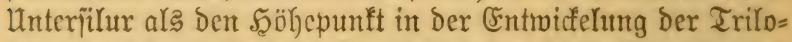

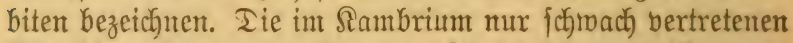
Bractiopoden nelymen mim raich an Formenreichtem zu (bejonders Drtbis und einzelne Epiriferarten, Taf.III, Fig. 15). Iazu gejellen fitch noch bie ভdjalent bon Rephalopoden aus der Srutupe Der Rautiliden; teils find es gerade, fitabförmige Formen (Orthoceras, Taf. III, శ̧ig. 1), teirక gefrïnmte unঠ Galb auffgerollte (Cyrtoceras, Taf. III, Jig. 2, Lituites). Bes fonders Yeitend und charafteriftifich fint die (S) raptolithen (Taf. II, Fig. 8), weldte in ben Eđhicferablagernugen fich in

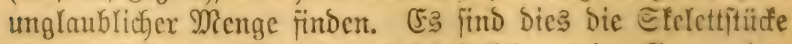
eines untoctannten Tieres (mafyrjocinlich) auts der (Sruppe ber

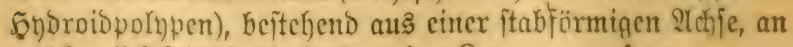

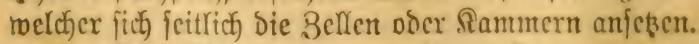

Das Dberfilur zcigt zwar im großzen ganzen now sine

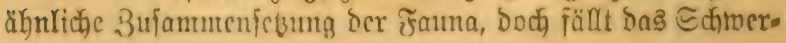


gemicht Dex Tiermelt nicht mefr auf die Trilobiten, melde ebenjo mie die Sraptolithen einen Rüdgang bemerfen Iaffen, jondern auf die anderen Tiergejdlecter. Die Nautiliden und $\mathfrak{B}$ rachiopoden ftellen fich mit grof̧em Yrrtenreichtum ein; Dazu gejellent fitch Die Seelilien in gröp̈ter (Entfaltıtng ibrex Formen. Sie maren zwar jobon in Unterjithur Durch bie fugel= förmig gebilbeten 8 yftibeen vertreten; im Dberfilur Dagegen

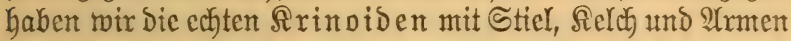
bor uns. Utuh Die Rorarlen bom Typus ber Tabulata und

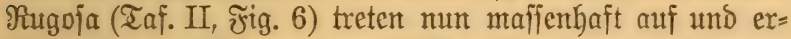
füllen einzelne 2Ublagerungen. Srrs bollitändig neute Frormen find fernex unförmliche und abenteuterlich gejtaltete Riefen= frebje (Gigantostraca) und Die exften Wirbeltiere in Siejtalt bon Jijchen za erwähnen; bicje waren jeboch nicht mit Schuppen, fondern mit jofildöörnigen Panzerplatten bedectt (Pteraspis).

\section{Devonformation.}

Diejes zmeite Silied Der paläozoifchen Formationen reift jich in Den für Das Silur ertähnten $\mathfrak{E c r b r e i t u n g s g e b i e t e n ~ a n ~}$ Die älteren Schichten an und bilbet Dort Die Direfte Fort=

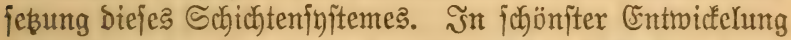

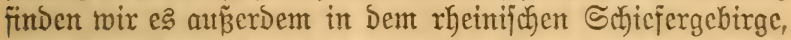
too es meift auf Tonjatiefer ber \$gyllitforntation auflagert. Somobl im Sieiteinscharafter twie in Der Fauna zeigt fitch Dą Debon jefr nafeitefento Den Silurablagerungen; auth jebst noch iiberwiegen Die Tonjchiefer, Siraumaden und Sanditeine, boch beteiligen fich auch Ralfiteite in weit größ̈crer ßerbrei= tung als in Dex Siluxformation. Während roir in Dem Silur

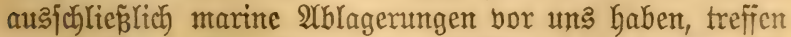
wix im Debon fchon Spuren bon Fejtländern, auf benen fich eine Sandflora ntit Sefäpifryptoganten cntwicfelte. In Der Tiertvelt zeigt fich eine ftete Weiterentwidfelung, mas jitch bes 
fonders bei ben Wirbeltieren geltend macht, weldye zwar auf

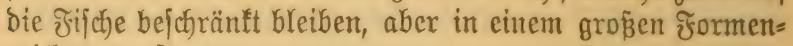
reichtum auftreten.

Man teilt bie Debonformation in brei Brieder ein, welche als Itnter=, Mittel= unto Dberdebon bezeidnet merben.

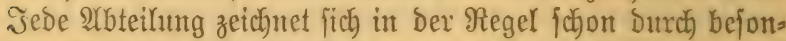
bere SHabirdung Der SBefteite aus, aber vor allem waren bei

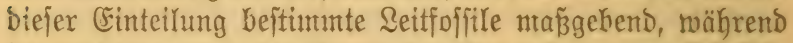
im groijen ganzen bie Franta ber Tebonformation fict febr gleidjunäßin bleibt.

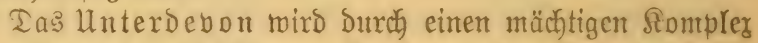
von fambigen Schichten, Mteift (Sraumadfen, Duarziten und Eantsfteinen gebirbet, in Denen Die Berfteinenungen utur idflectit crfalten finto. Itnter Den Sorallen bildoct das eigentünliche Pleurodictyum problematicum, bas faít immer nur als

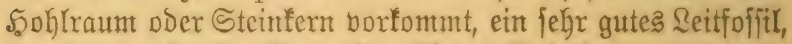
ebenịo twie einzelne Spirifer=2Yrten (Spirifer macropterus [₹af.II, Jig. 15] und cultrijugatus). Die Trilohiten uno Îti= noiben treten gegenüber Der Silurfauna bollitändig in ben

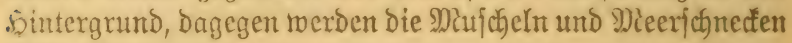
zahlreicher.

Dies macht jitch befonders in Der zmeiten IfGteiluntg, bem

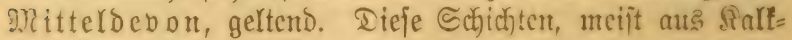

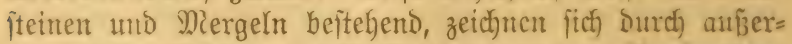

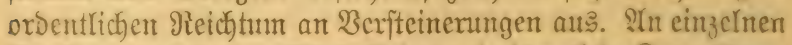
Siunften, wie in Der (Eifel, finden mir förnlifie ßorarren= ablage rungen, jog. paläbzoifche Sorallemifie, in twelcfen wir außser Dent zahlreichent Rornffen autch jonjtige Dieeres=

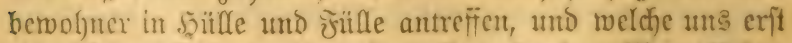
ein richtiges Birs von Dem inantaligen itppigen Diecrešleben geben fönnen. Ituter Den Sioraflen zeichnet fich bejontor: cine

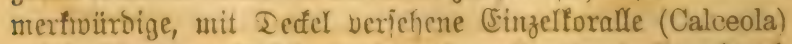
und Das häuffig zu ভtöđfen verfd, nolzene Cyathophyllum Durwh 
ছäufigleit aus. Bon Den ภrinoiben ift mur Cupressocrinus zu erwäbnen; ganz einzig fïr biejes frormationsglieb ba= gegen find die Bractiopodentgeichlecter Stringocephalus und Uncites. Aluch ?Rautiliben, fomofil gerabe geftrectte wie gefrümmte, jitnd febr häufig, und bazı treten noch eine

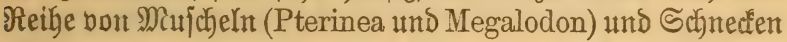
(Pleurotomaria); bon Den Tribobiten ift mux noch eine (Sattung (Phacops [Taf. III, F̂tg. 11]) bon Bebeutung.

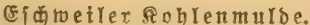

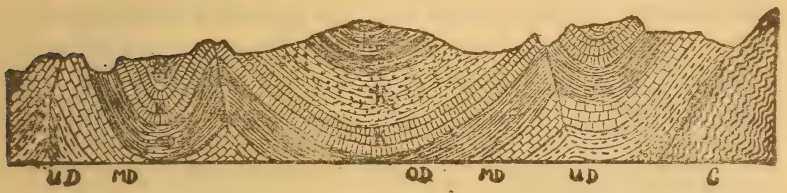

Deoon unb a arbou ber Ëifer.

C Rambrtum, UD Roblenzer Eđitchten mit \$leurodictbun. MD Eifler Salf mit Calceola und Stringocephalus. OD Dberbeonn mit Goniatiten umb firh-

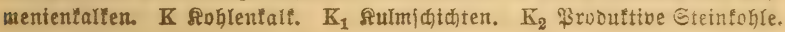

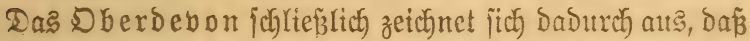
bie Rephalopoden eine jefr wichtige Entricfelung zeigen,

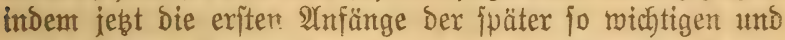
formentreichen (Sruppe Der 2 (mmoniten auftreten in (Sejtalt bon zmei (Bejchlechtern, Den Bs oniatiten (Taf. WI, Jig. 4)

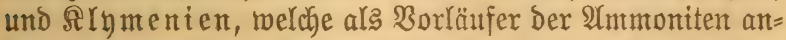
gefeben rerdor.

Die für bie Debonformation fo charafteriftifchen Fif che finden fitch uur felten in Den Deutfichen Meeresablagentungen, un fo häufiger Dagegen in Dent mebr litoralen Sanditeinen Des Sberdebons bon Sctjottland, Dem fogenanten "alten ro=

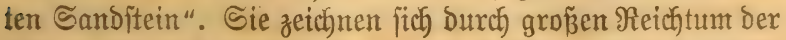
Arten aus, find zum Teil mit gropen \$anzerplatten bedect 
und Gaben bann eine ganz frembartige Ejeitalt (Coccosteus, Pterichthys [Taf. IV, ₹ig. 1]), oder aber ïe tragen ङchuppen, melche mit Dicfem Schmelz überzogen find; man bezeichnet fic als Sandiditiche.

\section{Stciufohfen= vber fiarbou-\{rormution.}

Die beiden jüngeren paläozoijchen ₹rormationen Gaben unter

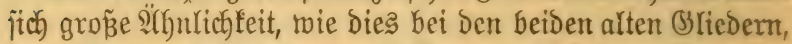
Dem Silur und Debon, ber Fall war. Esfon zu Ende ber

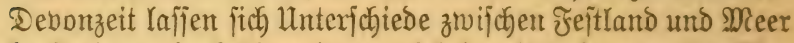
fonitatieren, Doch überwiegt noch bei weitem ber marine (5)a

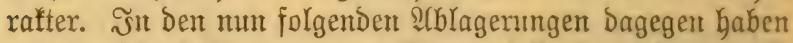
wir eine fcharfe Tremumg von Mcer und ¿anto und Demgemäß̃

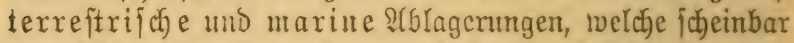
gar nichts mefre miteinander gemein faben, obgleidy jie geolo= gijoch gleichaltrig jims.

Die Meeresablagenungen bejtełen aus mädhtigen Ralk=

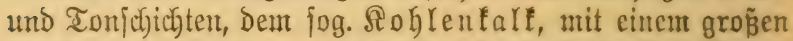
Reichtum an Seetieren, weldje eine fortlanfente Rette in ber Entroicfelung Der alten paläozoijd̄en formen baritellen. Un = ter Den Itrtierchen (尺roraminiferen) mirb bejoubers eine etwa exbjengrổe ralzenförmige ₹ornt, bie r'usulina (Taf. II,

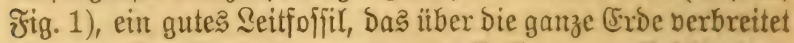

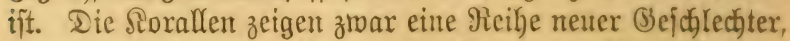
find aber Doch unch mit Den alten fornten aufs nächite bers manst. Eritaunlich iīt bie Entrwidefung ber Ecelilien,

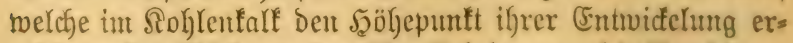

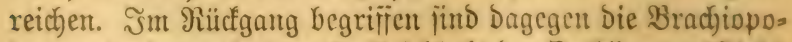

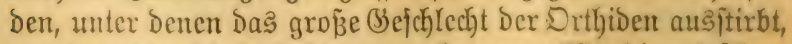

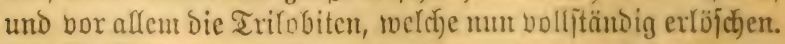
Die Rephalopoden find mir gering bertreten, es treteut mur menig neue ₹ormen aus ber (3ruppe Der Stmmoniten auf,

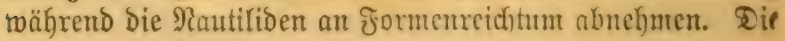


Mujcheln und Schnecten entfalten fich immer mehr und

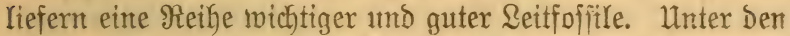

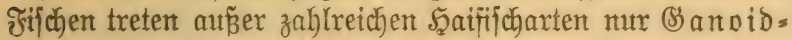
fifiche auf, Die Ŝnochentifote fehlen noch gänzlich.

Romment mir mun aus den Befficten ber tiefent Meere, melche Die §ohlenfalfablagerung Darjtellt, näher zux ehemali= gen Ritite, two bie Meere feich)ter inaren, unt zugleich viel mehr Material bom ₹eftland her zugefübrt befamen, fo finden mir bort $\mathfrak{2}$ (blagenuttgen bon ganz anderem (B) eiteinscharafter. Man bezcictunt fie als $\Re u\lfloor m=$ Formation. Diefe Bebilde

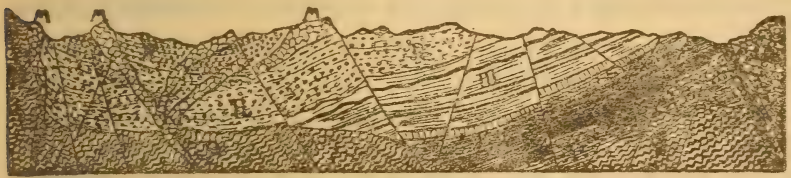

Saarbrüder Aoblentebier.

D Debonifies Mrunbgebirge. I Tiefite flöbleere Ganditeine bes Carbon. II Saarbrüder Stodmerl, probuttive Steiufoble. III ßfälzer Stndtwert,

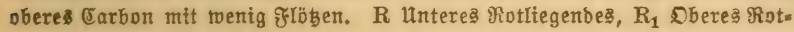
İegendes. M Melaphurgänge und Dedten. Bdst. Bunbfandftetn.

Dex Ifferznote beitehent durchgehend atts Ronglomeraten, Sand=

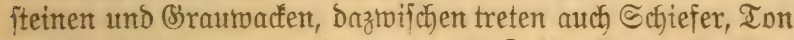

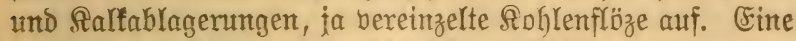
groß̈e ßetrefaftenarmut ntacht fich bemerffich), und mur menige mit bem Rohlenfarf gemeinfante $\mathfrak{2}$ rten bemeifen die geologifche (S) Brlieder Der Steinfohlenforntation Daritellen.

Tie produtibe Roglenformation ftellt bie oberite

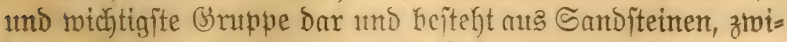
\{chen melchen Sodjiefertone unt Sohlenflöze medffellagern. Die 2 nnzahl fomohl mie bie Meächtigfeit Der Flöze ift eine foftranfente: bald find fie nur menige Bentimeter bict und 
Deshalb nicts abbautwürdig, bald aber fchwellen fie zut einer Meächtigfeit von biełen Mietern an. Tie Sohjenablagenungen find entftanden aus einer ïppig wutchernden frlora, welche bie fumtpfigen Niedenungen Deß Damaligen Feftlandes bedectte. Die $\mathfrak{X b l a g e n u n g e n ~ f i n t ~ i n t n t e r ~ m u l d e n f o ̈ r m i g ~ a n g e o r o n e t ~ u n o ~}$ auf berhältnismäß̄ig fleine Strecten, fog. Sohlenbecten ober Soflenrebiere, berteilt. Somohl bieje muldenförntige Lagenung, ats vor allem bie Entitefung Der Flöze auts \&ant= pflanzen und bie mit biejen borfommenden Rantiere madfen

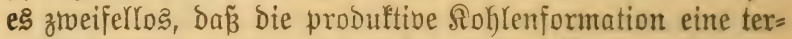
reftrifche Bildong ift, melche fich in Ciñzwaffertümpeln abges lagert fat.

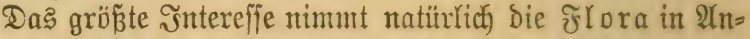

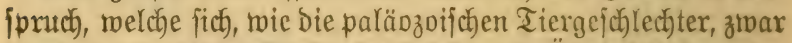
Durct) unglaubliche Maffenthaftigfeit und üppigfeit ber ein=

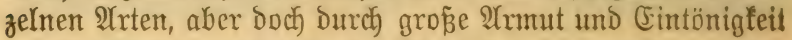

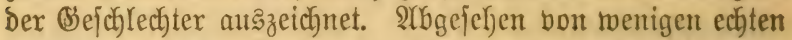
foniferen aus ber (Siruppe Der Arautarien, wutrde bie ganze Soblenablagenung vou Siryptogamen gcbildet; Die Sala marien oder Schachtergalme (Calamites, Asterophyllum und Annularia [T̃f. I, Fig. 2 uto 5]) wuckerten in ben

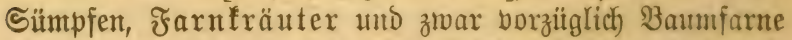
(Neuropteris, Odontopteris [Taf. I, Fig. 6], Pecopteris uno Alethopteris) waren mehr auf Den trodfenten 230 ben autge $=$ reiejen. Den größ̈ten $\mathfrak{A}$ nteil an Der Rohlenbiloung lyatten bie eigentïmlichen Sigillarien (Taf. I, Jig. 3) uno Lepido-

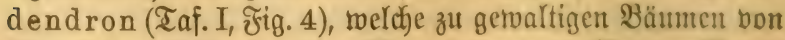
ïber $30 \mathrm{~m}$ şöhe herambudffen; Der ganze Ctamm ift mit Blattmarben befest, an melchen lange Nabeln faßjen, bie Wurzeln (fog. Stigmarien) find viclverzweigt unto verbreitet,

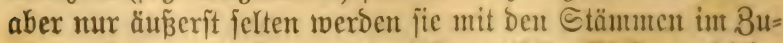
famtunbang gefunden. Die fnitematifaje Etellung bicfer

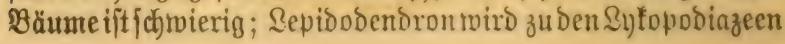


geitellt, während bie Stellung bon Sigillaria noch fodrwanfend ift. Damit ijt Die Flora fach exfdöpft, Dent alle tweiteren Sflanzengruppen, bor allent die angiofpermen Difotyledonen, fehlert noch; in weldfer Üppigleit aber biefe wenigen Arten wutberten, fann man fich faum voritellen, Dent unzählige Stämme und Blätter maren nötig, um folche Rohlenablage= nungen zu hinterlaffen, wie wir fte in Der Rohlenformation antreffen.

Tie Tiere treten neben Den झflanzen bollytändig in Den Sintergrund und gefören zu Den größ̄ten Seltenbeiten. Um

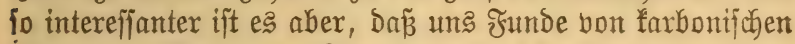
Spinnen, Sforpionen, Termiten, Şeujfyrerken und andern Infeften borliegen, toldhe ung beweifen, Dañ auch diefe

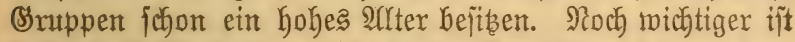

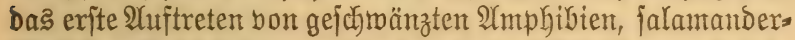
ähnlichen (sejchöpfen, weldje jedoch in Slelettbau noch fefr

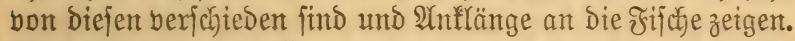
Man hat die ganze Simppe biejer alten $\mathfrak{A n t p h i b i e n , ~ w e l c h e ~}$ wieber in ber rrias aușterben, als S teg ofephalen (Taf.IV fig. 5 und 6) bezeichnet.

Die Berbreitung Der produltiven Sohrenforntation ift, wie focjon ertwähnt, auf einzelne Bedfen bejchränft; in I Ieutjobs land ift es Nieder= und Dberjobleften, Cachjen zmijchen Dem (Erz= und Mittelgebirge, Das ธaargebiet und Weftfalen, weldye bejonders gejegnet find; Heinere 2 ortomnntife find im Fichtel= gebirge, in Den Bogejen und im badijchen ऽctwarzmald bor handen, mogegen in Wïttentberg bie Tiefbohnung auf Stein= foblen refultatlos blieb.

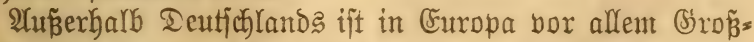
britantien Durch großßen fohlenreichtum auझgezeichnet, auf

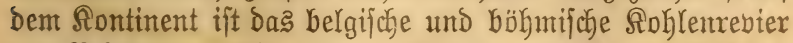
bon Bebeutung. Die maxinen 2 blagenungen (Soblentalf und

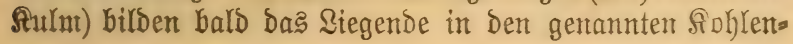




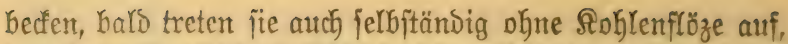
jomofl in Deutichland (ひichtelgebirge, Thitringen, S2arz), wie

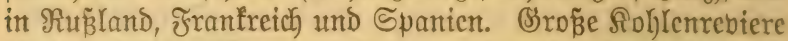

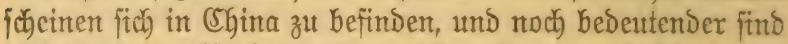
biejentigen bon Norbamerifa, welche zufammen ein $\mathfrak{A r e a l}_{\text {bon }}$ ca. 5500 Ditadratmeilen umfaffen.

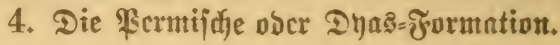

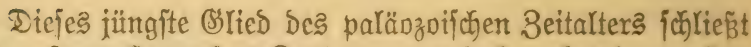
fich aufs crtgite an Das Sarbon an und betwahrt int groß̧en ganzen Denfelbent (Befteinscharalter und nabe bertwandte Dr: ganismen. AYtch fier herrichen Die litoralen und jogenamten terreitrijochen Bilsungen bor: Songlomerate, Sanditeine mit intergeordneten Soljlenflözen, Mergel uns Ton bilden bie Se=

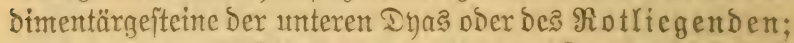

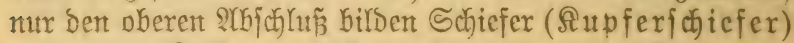
utno marine $\Omega$ alf $=$ und Dolomitablagentugen, ber jogentante Bechftein. Bejonders twichtig für bas ßotliegende find bie maffentyaften Eruptiogejteine, unter Denen Die Duarzpor= phyre bie erite Stelle eintrehmen. Diefe bilden nidht mur

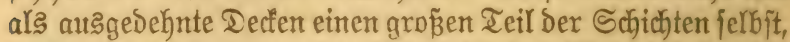

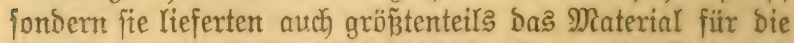
Songlomerate und Sarbiteinichichten. In zmeiter Sinte find

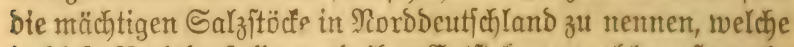

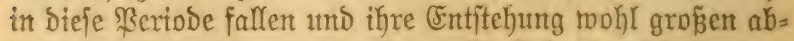
fluklofen Inlandjeen verbantten. Es find bie biele hunbert

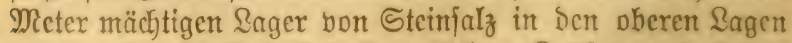
bon ben fïr bie Intouftrie fo roidjtigen Salifalzen begleitet,

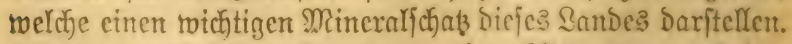

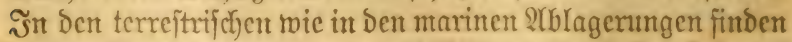
wir noch paläpzoff(c) Frautna und flora, aber beibe find auf wenige Formen bejchränft, wic iiberl)ant in ber Donas einc

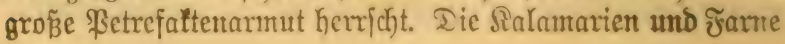


Der Sohlenforntation jïn auth noch im Rotliegenden bor= Gerrichend, nur bie Sigillarien und ¿epioodendron=2(rten find Durd echte Soniferen bertreten. Utnter Den niederen Seetieren Gerrichen im Bechjtein noch einige paläozoijche Brachiopoden (Productus) bor, aber Die Beit Der Nautilitiden, Gioniatiten,

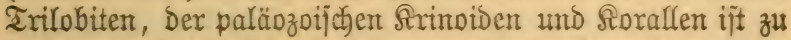

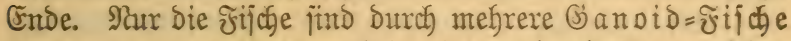
(Palaeoniscus) bertretent und die Stegof́ephalen erreiden Den S̋b̈hepuntt ifrer Ẽntfaltung (Archegosaurus, Branchiosaurus [Tafel IV Fig. 5], Melanerpeton 1t. a.). Cintent mejents licfen હchritt in ber Entroiffung Der Wsirbeltiere bezeidnet

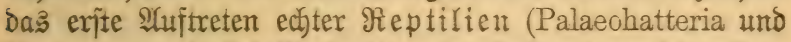

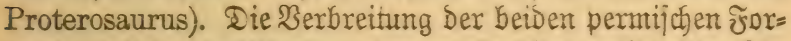
mationsglieder, Deşotriegenden und Bechjteins, ijt in Deutich=

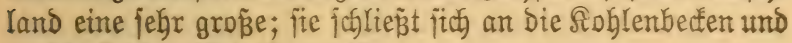
an bie fpätere Triasformation an.

\section{Drittes Zeitalter oder die melozoílchen formationen.}

Mit Dem SUftreten Der über Der Dyas gelagerten Schicften

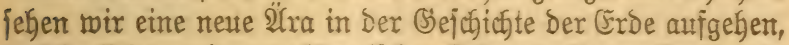

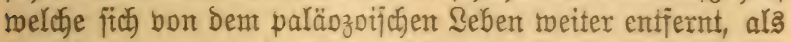

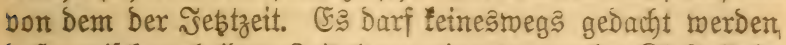

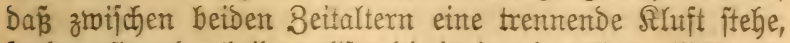

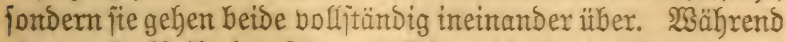

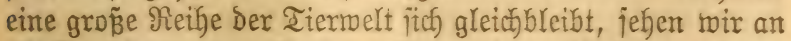
Stelle anderer ausgejtorbener 2 (rten eine grop̃e $\mathfrak{A}$ nzahl neuter auftreten und bazu fontmen nodf vollitänbig neute, Göber ent=

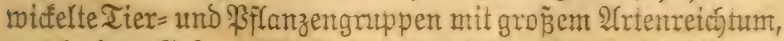
welche bem Bbejantbild ein yerärtbertes und mannigfaltigeres 
Bepräge verteihen. In Der $\mathfrak{P f l a n z e n t w e l t ~ g e h e n ~ z t w a r ~ n o c h ~ b i e ~}$ Befäp̈fryptoganten, wenn audch mit neuen $\mathfrak{A}$ rten burch), aber fie werden itbermogen bon Den Roniferen, und bazu gejellen fich noch bie eriten angiojpermen Difotylebonen, bie echten Saubförzer. Die in Den paräozoifchen Jormationen leitenden tabulaten Sorallen macken Den Seraforallen \$lą̧, Die Srino= iben, Brachiopoden und sautiliben bejchränfen fich auf

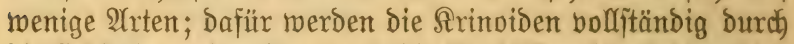

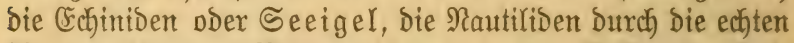
Âtmontten umb Belemniten bertreten. Ânitatt ber Tri= lobiten exicheinen num echte Srebie, bie Fifiche entfalten einen gropien Fonmenreicf)tum umb es gejelfen jïch) zu ben

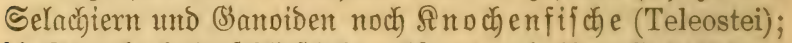

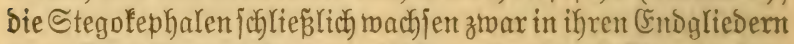
zu rief̣tgen Frormen Geran, erlïfchen aber bamit faton in Dem

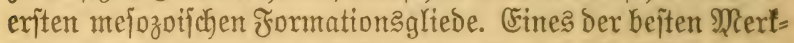
male bes mejozpificten Zeitalters gibt die Entfaltung Der

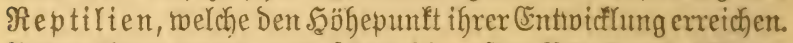
Neben thnen treten attd fichon bie erften $\mathfrak{B o ̈ g e l ~ u n d ~ S a ̈ u g e s ~}$ tiere auf.

So geitaltet fick Tier = und \$flanzentwelt in Der mejozoifden ßeriobe unendrich mannigfactjer unt formenreicher gegenitber Der früherent Beit, und twenn fie auth noch von bem Bild ber Jebtzeit tweit abmeid)t, fo fehen wir boch fidjon einten gantz wefentlichen S(f)ritt zur Âmuäl)erumg.

Was bie (jejteine anbelangt, fo läßst fich im allgemeinen

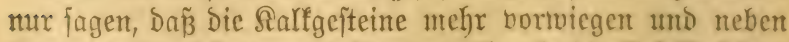
ihnen bie Sandfteine mb Tone, twäbrend bie ficfelfaltigen Dutarzite, (Sraumaden und Schicfer mur untergeorontet auf= treten. Buffentifde Entionen find twährent Diefses Beit= alters Eeltenfyciten und bafjer bon untergentioneter $B c=$ beutung. 


\section{Die Trias= Formation.}

Die Trias lagert als unterftes (S)ied Der mefozoifchen For. mationen auf ber Dyas und billot in Seutfaland robl die verbreitetfte allex Formationen. Şatten wir foron im Sarbon und in Der Dyas ftreng die terreftrifchen und marinen 2rblagenungen auseinander zu halten, fo ijt bies in gleichem

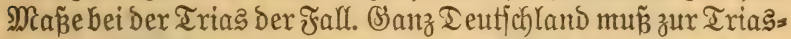
periode ein flaches Rüftenlanto getwejen fein, in welchem Durch geringe Dzzillation Des Rontinentes bald jeidyte Meere fich auझbreiteten, bald grop̃e Binnemjeen abgejcynürt murben,

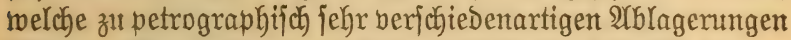
fïhrten. Banz anders geftalteten fich die Berbältniffe Da, wo tiefe Meere ihre Nieberforläge Ginterlafien haben, wie wix dies in Den צrpen treffen. Während fich in Der Deutjchen Irias Sanditeine, Salfe, Salzftöle und bunte Mergel mit terre=

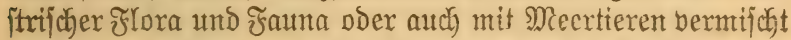
borfinden, treffen wix in ber alpinen Irias fait mur Salfe und

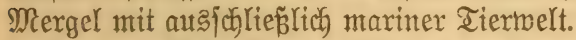

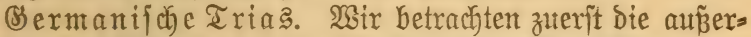
alpine beutjche Trias, weldye Durch ibre flare Sriederung in Buntianditein, Miujchelfalf und Reuper zum Namen Irias Beranlafiung gegeben hat.

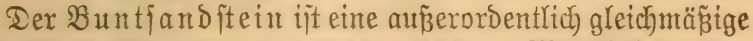
Sanditeinablagenung von $300-400 \mathrm{~m}$ Mäcbtigfeit; Der Sandftein ift meift dumch (sifangebalt rot gefärbt und befïbt eit gleichmäpítges feines Sorn, we ganz bejonders gut eignet (Şeidelberger Schlopis). Jür Den

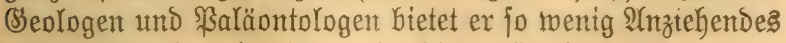

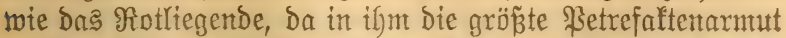
Gexricht. Nur hite unto Da bezeugen Rflanzen (Voltzia) Dder Die Fälyrten bon riejigen Stegofephalen (Chirotherium) und

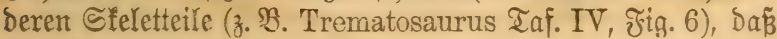


bas Reben nicht volfitändig erlofdyen war. Srit in Den oberiten

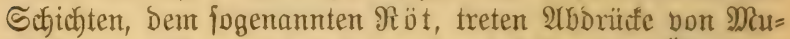

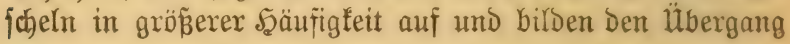
zมm

Muf

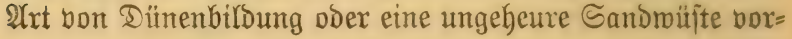

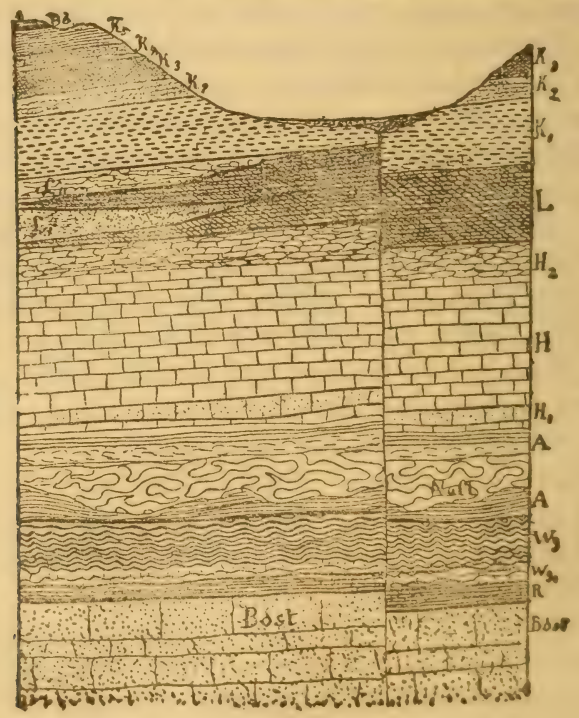

(C) roäbifde זrias (Untergrmo non Stuttgart).

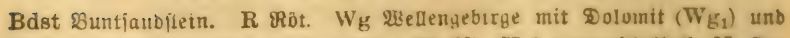

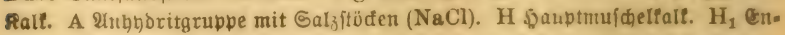
Irtniten= $\$$ orizont. $\mathrm{H}_{2}$ Ceratites nudosus- jorizont. L \&ettentoble mit $\mathrm{L}_{2}$ \&etten=

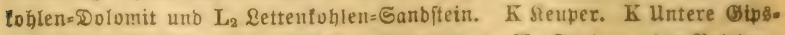
mergel. $\mathrm{K}_{2}$ Sdilfiandftein. $\mathrm{K}_{3}$ inute BstpsmergeL. $\mathrm{K}_{4}$ Stuber. ober Belobon. fanoftein. $\mathrm{K}_{5}$ Biolette Mrergel (8anflobon). Bd Bonebeb beß Pät. 
itellen, melche mun yon einem jeichten Meere überflutet tourbe.

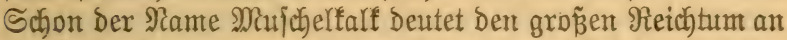
Berfteinemungen an, welche meijt aus ben zahllojen Schalen von Maricheln bejtehen, unten Denen bie glatten Trigoniaarten, Die jogenannten My op horien, Die wichtigiten fint. Forallen und Spongien feblen faît ganz, Dagegen tritt eine Sirinoibens art, Der Encrinus liliiformis (Taf. II, Ĵig. 9), in eritaunlicter Menge auf und jeine ๔tielglieber bilden ben Trochitentalt. Bon Den Rractiopoden zeichnet fich die Terebratula vul-

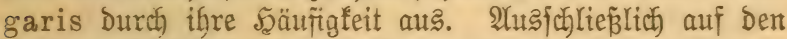

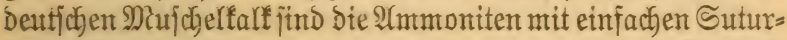
linien, Der Ceratites nodosus und C. semipartitus, bejdyräntt. Auch Saurier.bebölferten neben Den jpärlichen fijchen Das Meer und zwar waren es eigenartige Formen mit gebrungenem ¿eib, fleinem $j$ chlanfen Ropje auf eintem langen \$2als und furzen, zum

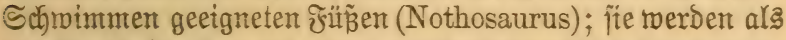

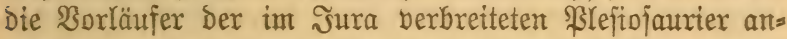
gejeben.

Sn Sübdentichland, befonders in WHuttemberg, finden jitch

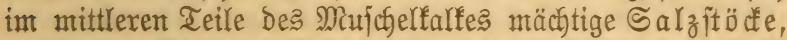

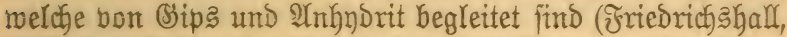

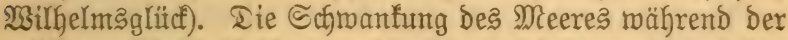

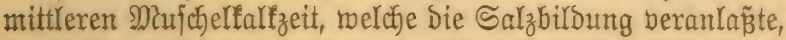
madjt fich aud in ben andern Deutfichen (sebieten geltend und Gat Deranlafiung gegeben zu einer Dreiteilung in unteren Mujafelfalf oder Wellengebirge, mittleren Mujchelfulf oder

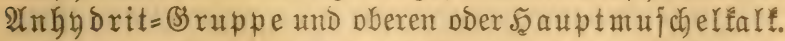

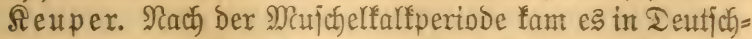
Iand zu neuen gropenen Betwegungen Des BoDent, weldbe Die Dif̈enen Meere berorängten und an Deren ミtelle ミagunen und groje abflup̃loje Binnenjeen febten. Die Geiteine, merche fich Gier abjesten, bilden Den buntejten $\mathfrak{B e c h j e l}$ Dex Hblagerungen, welchen wir überfaupt in einer Fomation fenten. Cando= 
fteine, Dolomite, Salfe und bor allent Mergel mit viel Sips und Steinfalz in allen möglichen Farbentönen lennzeichnen bie Situperjechichten. Sie jind im ganzen terrejtrifoch Siatur; bafïr fprecken bie vielen \$flanzenrejte und bie Sandfaurier. Die Seen jelbit maren, wie z. B. Kente noch Das Tote Meer, gefïllt mit einer überjüttigten \&öfung bon Salz, in welcher ein Seben für Seetiere nicht mehr möglich mar. Iaher finden wix auth feine marine Fauna Diejer Binnenmeere, fondern nur bie ipärfichen, bom Rande hergefdymemmten lüberreite pon ¿anobewohnern.

Die \$ffanzentolt ift bejonscrs reich entroidelt und zcichnet fich Durch Das 2luftreten bon (Equifeten (Schachtelhalmen)

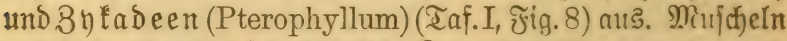
und ङchnedfen find jebr ipärlich; Arrindiben, Brachiopoden und Sephalopoden fehlen bollitändig. Unter ben Fifchen ijt be= fonders ein Tipnoer (Durd) Sunge uns Riemen atment), ber

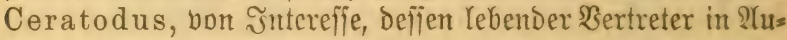
ftralien entbectt mutrde, ferner ein fleiner (Sandidfifch), S e m i onotus (Taf. IV, Fig. 4), und eine Altzahl bon fra a fif cha r ten

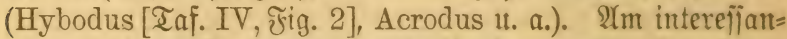
teften find die Bewofner Des Frftlandes, mafre llngebener an Bhumpheit und (Sröß̈e. Bon Den 2 mphibien faben twir bie

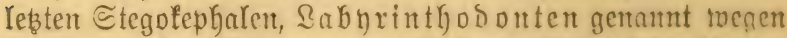
ifres eigentiunlich bertoorreneu Bahnbaucs; ba lebte Masto-

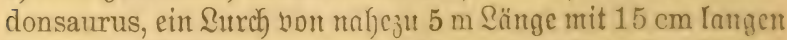
Ecfzüfnen, Cyclotosaums uा Metopias mit mefir alङ $2 \mathrm{~m}$

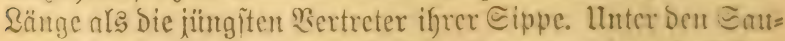
riem finden wir noch ??othofauriden von ben zierlichen, faum $30 \mathrm{~cm}$ langen Formen bis zu Micjen bolt 4 mo $5 \mathrm{~m}$. Bejonder eigenartig f̈nd die frolobilartigen gepanzerten Picp=

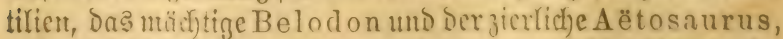

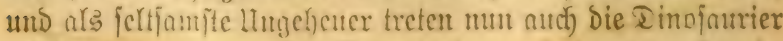
mit ben gegen $7 \mathrm{~m}$ groficut $Z$ a n clod on anf, eincut ganzfremo. 


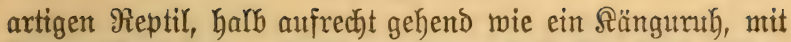

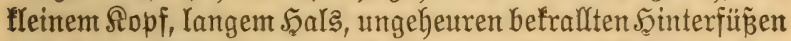
und einem Stübifabmanz. In ber oberiten Schicht twurben in Wüttemberg auch bie eriten Spuren bon Säugetieren entbectt (Microlestes und Triglyphus).

Whie beifolgendes \$rofil zeigt, wird der §etper in eine

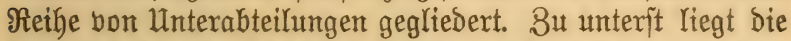
¿ettenfohle, hauptfädhlich aus Mergeln mit fleinen Rohlen= flözen, Pflanzenjanditein und Dolomitifchen Salfen bejtehend. über ifr folgt ber bunte ßeuper, Deffen unterftes Biried Sitpmemergel bildon; auf ifnen lagert ber Schilffand itein, erfitllt mit (Equijeten; es folgen abermals jegr farbenreiche, meift rot und grïn gefärbte (3)ip a mergel (rote Wand), Dann weizer Sanditein, jogenannter Stuben= oder Burgfand=

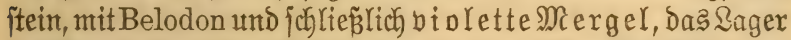

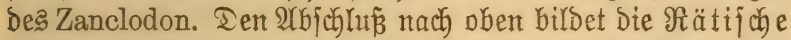
Stufe, in Deutfolland gewöhnlich als eine Inochenbreccie bon menigen Bentimetern ('̧onebed) oder ars maxiner Sand= ftein mit einer leitenden Mirfichel, Der A vicula contorta, entmiccelt. So unbedeutend biejer Şorizont ift, fo ift er Doch bei Der ßarallelifferung Des auteralpinen Яentpers mit Dem alpinen Settper von groß̌er $\mathfrak{S}_{\text {Sidgtigfeit. }}$

Arpine Trias. Sbantz berjobiedenartig bon biejer jo= gentanten Deutfichen Trias find Die gleichaltrigen 2(Glagenungen in Den AYrpen, bie fogenannte alpine Trias. Dort fluteten während ber ganzen Triagzeit tiefe Mieere, in weldefen eine

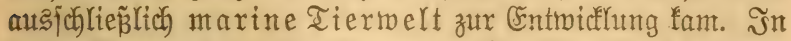
biefen Meeren Yebte eine Menge bon Soraflen, Bractiopoden,

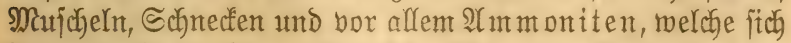
inmer mefr bon Den paläozoif dyen Formen entfernten und Die Beit Der föchjtent Entroicflung der Afmmtoniten in ber Jurra= periode borkereiten. Arker autch fothon zut Iriaszeit mathte jith offenbar die Untuhe im Untergumbe geltend, welche fpäter 


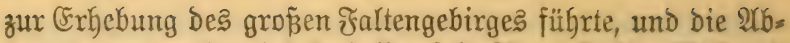

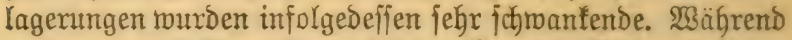
an ber einen felitgen Stelle fich den Sorallen Belegentyeit zur

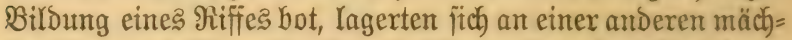

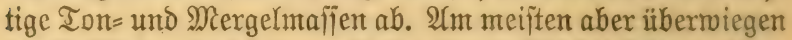
bie Salfiteine, welche zum Teil eniorme Mädhtigfeit erreichen und fich häufig Durch) \$etrefaftenreichtum auszzeichnen, wäh= reno bie gleichfalls febr mäctitigen Dolomitgejteine fich fegr arm ermeijen.

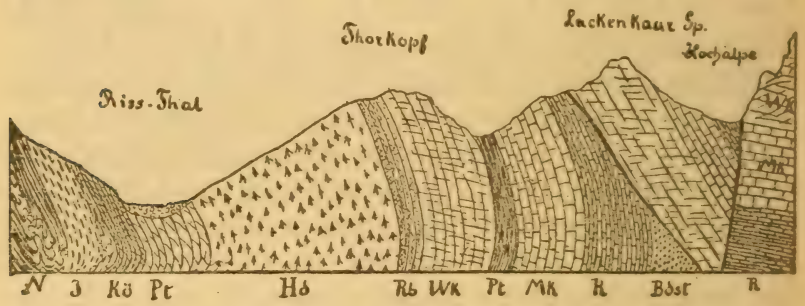

IIpine Trias (Rartwendel=(Sebirge).

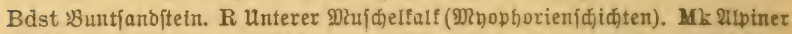

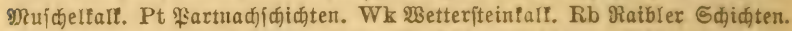

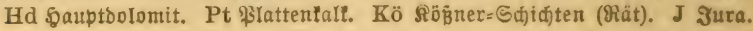
$\mathbf{N}$ sireibe.

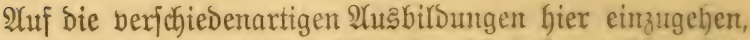

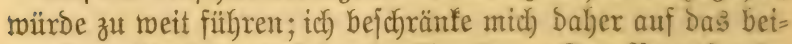
gegebene \$rofil und folgende 2 lngaben: Ter $3 u n t ; a n d=$ ftein und ber untere Miujeclfalf find in grojen ganzen ähnlich twie in Dem auf́cralpinen (Sebiet. Turch eincit Dier= gelforizont (Partna $d$ = umb St. (Eaffianer Ecfichten) ge= trennt, forgt eine bis $1500 \mathrm{~m}$ mäctige fialfablagenming, Der

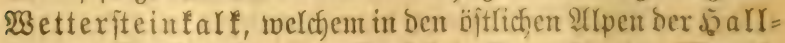

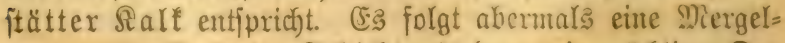

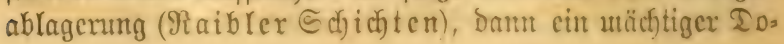




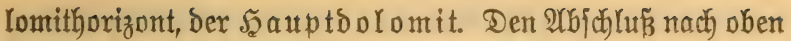
bilben die rätifachen Schichten, twelche in Den Irlpen eine ungentein grop̧e Mannigfaltigfeit uno Mëchtigleit zeigen, aber die Avicula contorta mit Den auperalpinen oberiten feuper= janditeinen gemein baben.

\section{Die Suraformation.}

Mit Ende Dex Triasperiobe merben in bex germantifien

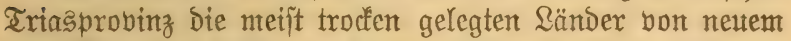
vom Meer itberflutet, welches ans eine mächtige, meift rein maxine 2rblagentng Ginterlafien bat in Form bon Salf und Sandfteinen, Mergeln und Echicfertonen.

ङs ift interefiant, Diejes übergreifen des Meeres über das

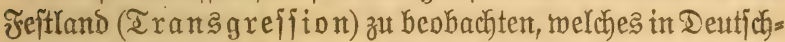
land bon SW rach NO und $O$ bor jith ging. Sn einter mäch tigen fette, Dem S̈tragehinge, Daङ fitch von Dem Schweizer Sura bis zum Fichtelgebirge Durchzieht, find uns die Fornta=

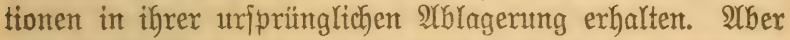
auch in Norbent bon Deutichland feblen fie nicht, mur tretent fie nicht in einem Derartig gejolofifenen Bujammenhang auf, jondern find auf wenige Dofartïäten bejchränft. Die mit Den

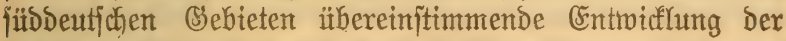

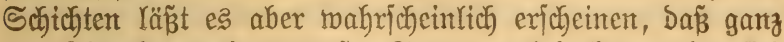
Deutichland bont einem grofien Surameer bedectt wax, Das fith

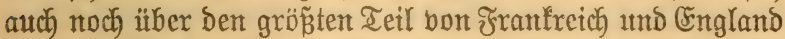
exitrecte. Siegen Ende der Suraperiobe fönten wir eine alls

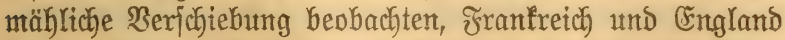

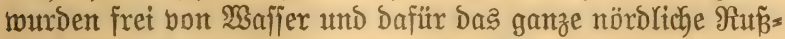
land überflutet.

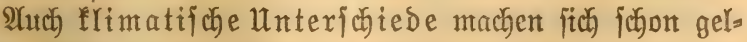
tend, indem bie nörolidben borealen Meere eine bon ben jüts= richen mediterranen und äquatorialen \$rovinzen berfdjiebene Tierwelt beherbergen. 


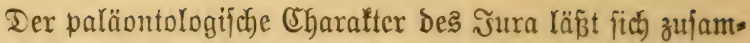
menfaffen als bie $\mathfrak{B}$ lütezcit ber $\mathfrak{A}$ mmoniten und $\mathfrak{B} e=$ remniten, fotvie ber grofien Meerjaurier. Bu biejen gejellen fich noch Bertreter fait aller im Meere lebender Tier=

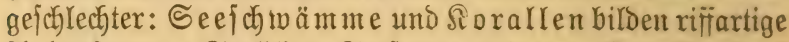
$\mathfrak{A}$ nbäufungen; Seelilien, Seejterne und bor allem Seeigel find in grop̧er 2 (nzahl bertreten; Dazu die formenreidjen $\mathfrak{M}_{\mathfrak{u}}=$ jheln und Schneden, Die frrebje und bie Fijche aus Der Siruppe der (Siano id fif che. Auch die Bemohner Des Zandes ftnd unt jotwobl in Den Siuftengebilden Der Solntyofer ミschiefer, wie bor aflem in Den mächtigen terrejtrijchen 2rblagerungen

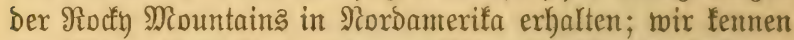

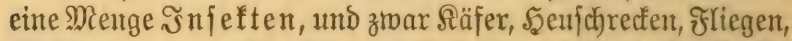
Spinnen u. a., weld)e fich zum Teil Durdh bebeutende Brï̈вe auszeichnen und an die heutigen in Den Tropen Yebenden

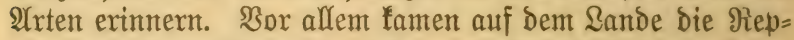

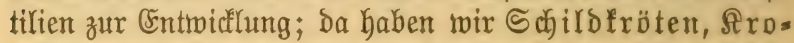
todile und Saurier, wie fie heute auf Renjeeland reben (Rhynchocephalia), Daneben aber auch bollitändig ans= geftorbene Srmppen, wie bie abentenerlicken Flugiaurier (Pterodactylus [Taf. IV, Jig. 8] und Rhamphorhynchus) und bie fdyon befdriebenen $\mathfrak{D}$ iubfaurier. Reksterc werben befonders in Den amerifanifocten 2 blagenungen gefunden und erreicher Dort Die faum glaubliche (Sröß̈e von $24 \mathrm{~m}$. Die Schenfelfnochen biejer Ricjen haben allein eine Şöbe von 2,3 m (Atlantosaurus). YYuch Der exite gefiederte $\mathfrak{B o g e l}$ (A rchaeopteryx) und flcine Säugctiere, welche in feine ber jest lebenden (Sattungen eingereiht twerden fömen, wurben in Europa mo Ŝlurerifa gefumben.

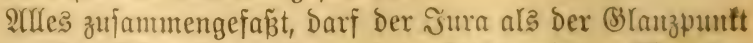
Der mejozoifechen Formationen betractetet werden. Man bat bie Suraformation in Drei (3) lieder geteill, weldse nach ifrer

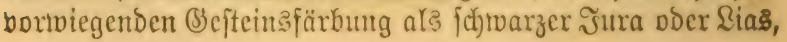




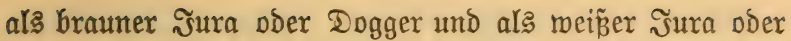
Malm bezeichnet merden. In allen brei Sriedern werden wieder eine $\mathfrak{A}$ nzahl Interabteilungen oder Sorizonte unter= fatieden, welche forobl burch ifre, Seiteinart, wie Durch ifre ßeitfofitle ficher auseinandergehalten merden fönten.

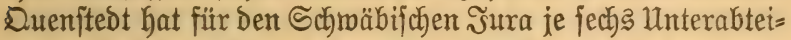
lungen in jedem (s)liede eingeführt, weldye ex nach dem grie=

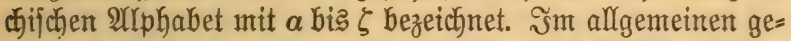
nügt eine Einteilung in unteren, mittleren un oberen ミias,

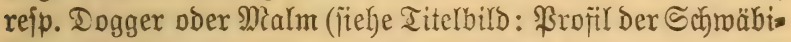
j(hen $\mathfrak{A}$ (n)

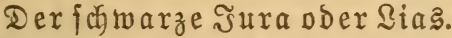

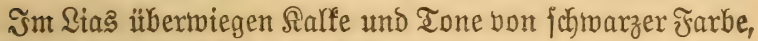
mährend die Sanditeine zuriatreten. Schon in den unters ften אalfbänfen Gaben twix eine Fülle von $\mathfrak{A m m t o n i t e n ~ ( T a f . ~}$

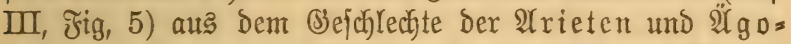

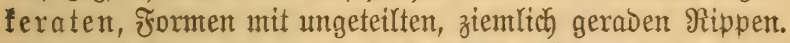

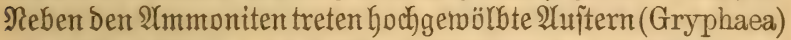
in zabrrofer Nenge auf, toälyrend Terebrateln und Belemtniten noch fichrach bertreten find. In Den englifichen UYblagerungen finden fich fatjon fier bie zablreichen Meerjaurier und Fijobe, welche in Teutichland exft in Den höberen Stufen lyäufig wer= Den, Dagegen in Den untern Lagen fehr jelten find.

In Den mittleren, meift Durch Mergel und Ton gebil=

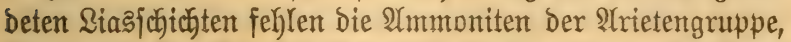
Dagegen ftellen fich bie $\mathfrak{A}$ maltheen ein, Formen mit ge= jutrungenen Rippen und einem zopfartigen Sitel auf Dem Fücfen. Uuch die Belemniten (Taf. II, Fig. 8 und 9), Die fingerartig gefildeten (Endftüfe( (jog. Phragmofone) Der Schulpe bon Tintenfijcben, treten nun in zahlrofer Menge auf, begleitet

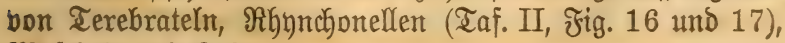
Muipheln und Sçnedten. 
Der obere 2 ias zeigt uns eine 2rblagerung bon ausges

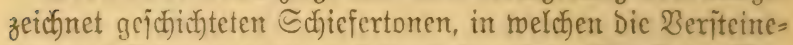
rungen fait inumer plattgebriictit erbalten finto. Cine fleine $M_{u} u=$ jchel (P o sid o n o m y a Bronni) iīt bas häutigite @cilfofifit; unter

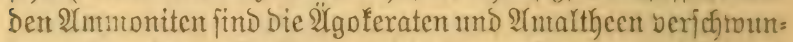
ben und an beren Stefle treten bie $5_{2}$ a rp o feraten mit Sichel=

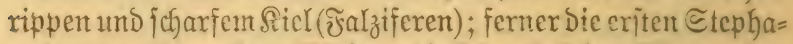
noferaten mit nundem Sï̈cten und fict gabelnocn Sitppen, Da= neben Dic PGyllof feraten umb \&ytoferaten mit glatten llmgängen

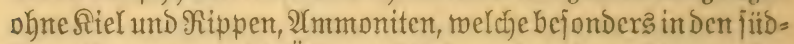

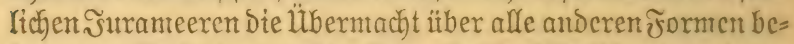
- fommen. Atud eine prad) tnolle gropie Seelilie (Pen tacrinus

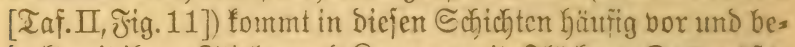

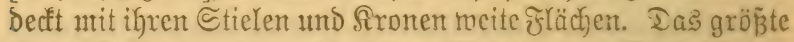

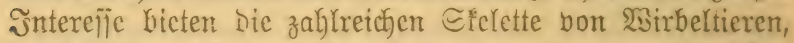
welche Gier nicht berfallen, fondern in ifrem ganzen $8 u=$ fanmenlyang borfontmen und cin flares billo des frïferen

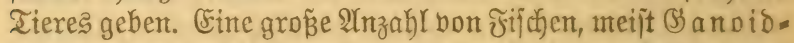

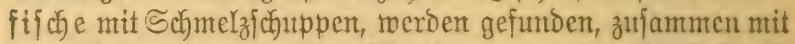
ben Sleletten bout grof̉en Meerfauriem. Ter häurigite ift

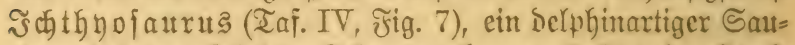

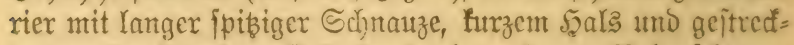

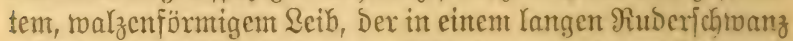
endigt; Die Filïe finto zum Schinimmen eingerichtet unt

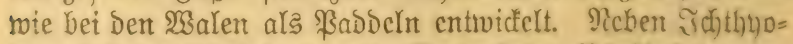
faurus findet fid bejonders in (England Der Frejiojaurus,

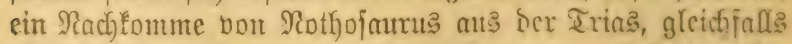

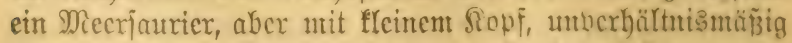

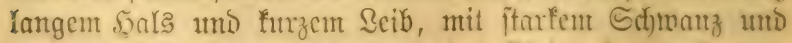

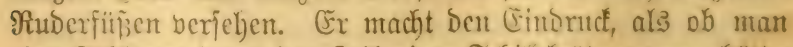

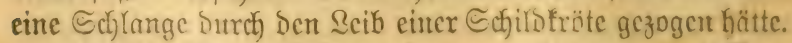
Der britte in Sinte ift Te Te ofauru 3 , eine gepanzerte firo: tobilart mit einer Sänge bis zu $5 \mathrm{~m}$. 


\section{Dex braune Эura ober Togger.}

Die Bejteine biejes mittleren Эuragliedes beitehen meift aus Tonen, Sanditenten und Den febr charafterijtijofen Dolith=

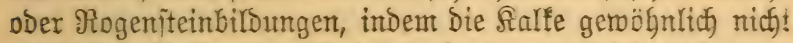
als bidjter אalf ausgebildet fint, fondern aus feinen fonzen=

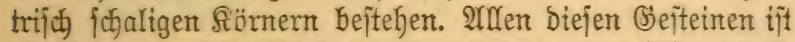
eine braume rojtige Färbung eigen infolge bon (Fijengebalt,

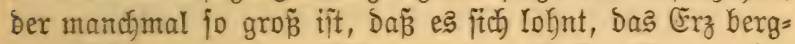

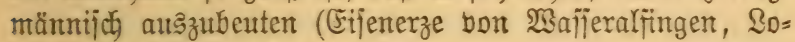
thringen und Surremtburg).

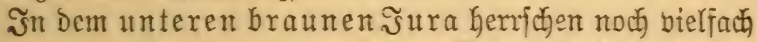
Tone bor, auf melchen bann int beutfigen כึtratgebiet eifen=

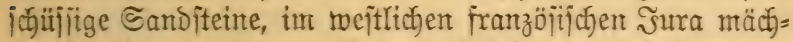
tige Solithbild ungeri (Feauptoolitg) folgen. Tie \&eitfojijtle werben rober bon Âmmoniten gebildot, unter melchen ficharf= fielige 5 arpoferaten herrfiend jind (A mmonites opalinus und Murchisonae). Lnter ben Muidfeln geminnt bas (bejeflectt Der Trigonien an Bebeutung, währent bie Saurier und Fithe, forvie bie Belemniten und frrindiben in Den Sintergrund treter.

Ter mittlerebraune Jurahat edte Stephanoferas=

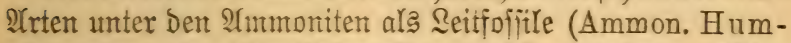
phresianus). Tie Belentniten machjen zut ben gröšten ซ̛̉nruten (Bel. giganteus) Geran, und unter Den Mitidjeln er= reichen Ditreen und Trigonien eine befonders reiche (Ent=

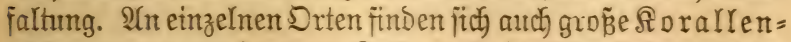
ablagerungen in Den Salf = umb Dolithgeiteinen biejer jorizonte.

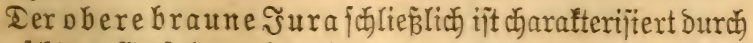
bie foböne Entfaltung Der Stephanoferaten, mit Dicfen, nahezu fugeligen formten (Ammon. Macrocephalus) ober rrich berziert Durch Sntoten uns Tornen (Ammon. ornatus). 
Reben Den Ânmoniten treten zahlreiche fogöne Terebrateln, Mujcheln, Schnedfen und Belentiten auf, jo daż bieje

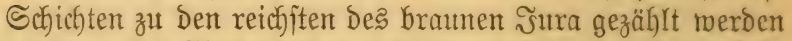
bürfen. Tie Mafrofephalerichichten beitehen meijt auts febr

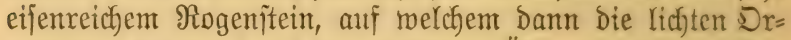
natentone lagern, Den faum merf́baren Übergang zum mei⿱艹̈en sura bitbento.

\section{Der weiñe Jura oder die Malmformation.}

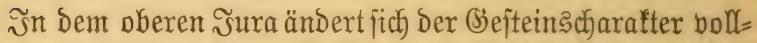
ftändig, Die Sanditeine, Dolithe und Schieferiteine merden berbrängt, und an ifre Stelle treten lichtfarbige, reine und tonige Sialfe und Dolomite, rwelche Durch ifre langjameres Berwittern Bexanlajiung zu Dem Steilabfall Der Suraberge

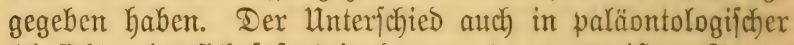
Şinficht zeigt fïch fofort in Dem unteren weizen כૅura.

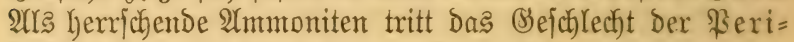
iphinlten ober \$lamulaten auf, Die Belenmiten treten in

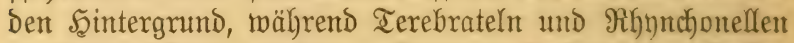

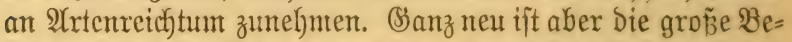

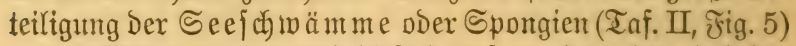

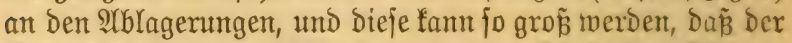

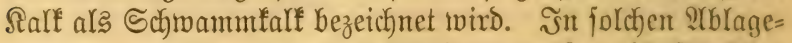
rungen treten Dann auch (Echinodermen, fowoht Eeclitien (Pentacrinus und Apiocrinus [Taf. II, fig. 10]), wic Sce= fterne und bor allem bie fchönen Seeigel (Cidaris [T̃af. II, ซ̌ig. 12 und 13], Diadema, Echinus u. a.) in groß̉er Mientge auf. Tie ganze Fauna twirb baburdh gegeniiber bent unteren Эิura cine volfitündig neue.

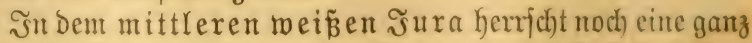
ähuliche 3 ufammenjekung Der Fauma, mur baj jich zu ben Berifphintten noch tweitere SAmmonitengefd) (echter reihen, bon Denen bie flact)en Dp pelien ober frlexuofen und Die biffen, mit 


\section{Drittes Beitalter.}

einzelnen Anoten berjebenen $\mathfrak{A}\{$ pio oferaten oder Inflaten bejonders wiattig find. Aluch fier find Spongientalfe fehr

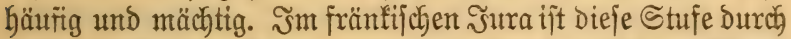
gropie Iolomitmaffen bertreten, meldye die fabönen Berg= formen und Şöblen Dex fränfifdyen Safweiz bilden.

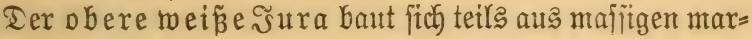

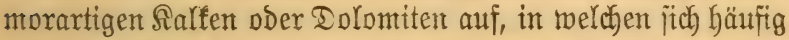
müchtige \{rnbäufungen bon Aorarlen (z. B. Thecosmilia, Iaf. II, J̛ig. 7) finden, bie an Stelle Der Spongien treten, teils aber auch aus Dïmnplattigen Salfen mit jebr feinem forne, weldye als Solnhofer Schiefer oder Plattenfalfe itber= all befannt find. Diefe Schiefer maren zur Exbaltung ber Ïberrefte auferordentlich geeignet, und po finden wir in ifnen nicht nur Întmoniten, Tintenfijche, Mujicheln, Sirebje, Jifiche und Saurier auf Das befte erfalten, fondern fie berwafyten uns jogar die SYborïcfe fonjt ganz vergänglicher Tiere, wie der Snjeften und Würmer, ja jogar Der zarten Meerquallen. Wie jobon ermähnt (Seite 78), itellt Die Fauma eine Mijayung bon

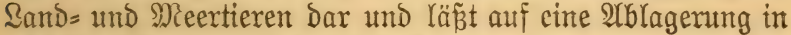

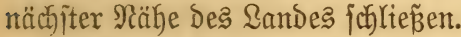

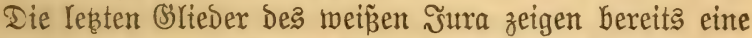

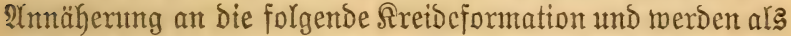
Tithon bezeichnet. Diejer Sharafter macht fich bejonders in Den mediterranen Arblagenungen geltent, wie wir fie in Den ZYpen und Sarpathen treffen. Dort bildot Das Tithon eine

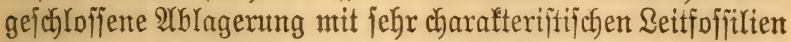
(Terebratula diphya und Ammonites elimatus).

\section{Die Sreibeformation.}

Der Saie ift gemöhnt, unter Den Sreidegefteinen fich nux ienen meip̈en erDigen Salf, bie Sdyreibfretbe, zu Denfen; Das ijt jeboch ein ganz faljcher Begrifi, Denn die Schreibtreide bildet nur einen ganz geringen Bejtandeil ber angemein wect)= 
jelnden Befteinsarten in Der Sreideformation. Biel bäufiger

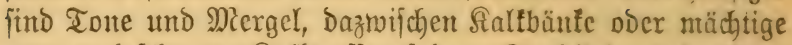
graue und fchwarze Salfinaffen, fodan Sanditcine und nahezu lofe, Käufig grïn gefärbte ভande, fog. (Silautonit= ober Brïn=

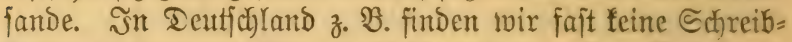
freide, fondern nur Mergel, Salfe und bor allem (Srïnjande und Sanditeine; Yeiztere bildon bie mächtigen fireibeablage=

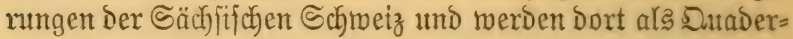
fanditein bezeichnet.

Jm großjen ganzen lyerrichen in Der Sreide Die marinen Mrblagerntugen bor, Doch feglt es atth feinesmegs an bracti=

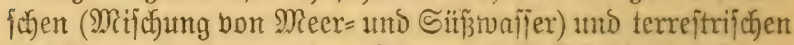
Gebildon. In Dex marinen Tierwelt läp̃t fíd ein langjanter

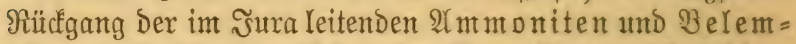

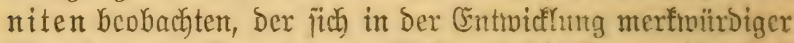
Berrformen łennzeichnet. Wälrend bie Âmmoniten bes Sura eine in einer Ebene allfgerollte Schale baritellen, und

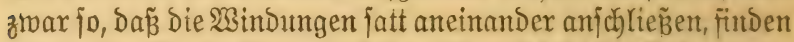
twir in Der Sreide Formen, bei weldyen fitch Diefer Bufantmen= Gang löft, bald mur in Dem Yebten llmgang (Scaphites [Taf.

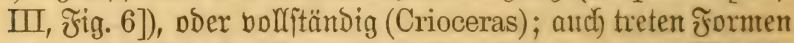
auf, welche fpiral aufgemumben (Turrilites) Doer bolfitändig

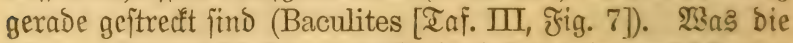

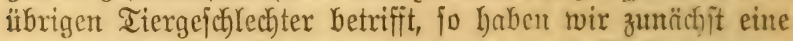
enorme Fitfle bon Foraminiferen und Radiolarien

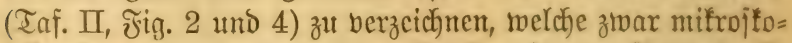

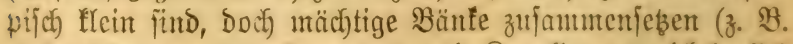
Edfreibfreide). Die Spongien und forallen entwidfeln fict) tweiter und crreidjen einen groß̈en Formenteichtum. Bei Den (Ec) in Den F̧intergrund gegenüber Den Secigeln, unter melityen neben Dent regulären Bibariten bie irregulären (bilateral fynt= metrifchen) frormen (Micraster [Taf. II, থig. 14], Spatangus 
und Echinoconus) bortwiegen. Tie Brachiopodengefdrecter

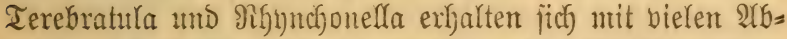
arten auf Derjerben Şöbe wie in Șra. Tie 2Ymtähentng an

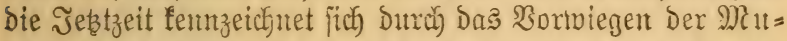
jकeln und ๔chneden, tweldie in Den Bordergnmb aller Schalentiere treten. Ebenjo ijt Diefe Armtähenng bei ben Fijchen zu beobadyten, unter ineldyen niajt melyr bie (biantoid=

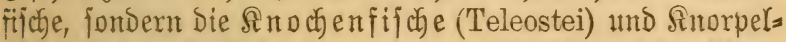

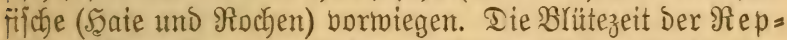
tilien ijt borüber; zwar futs noch alfe jurafitichen (Sejchlecter Durch einzelne Fornten vertreten, Die zun Teil zu riejigex Srröß̈e herantwachjen, aber fie bezeichnen mur Die Enoglieber

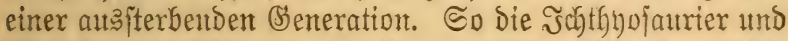
ßlejiojantiex (Pliosaurus mit gegen $10 \mathrm{~m}$ ¿änge) und die Dinofaurier mit bem $7 \mathrm{~m}$ gropen Iguanodon, Megalo-

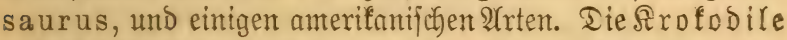
und Schild föten allein entwicfeln fitch greichmäñig weiter und bazı tritt ein neuer langgeitrecter Meerjaurier, Mosasaurus, Defien getwaltige Überrejte bei Miaaitricht uns

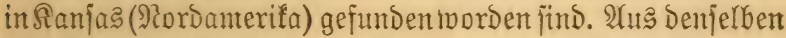

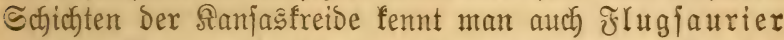
mit $6 \mathrm{~m}$ Spannmeite (Pteranodon) uno $20 ̈$ ger, weldye fitch Durch Die Bezahnung Der Siefer (DDontornithen) auszeiduten. Uruffallendertweife find bie ऽäugetiere noch ebenjo felten twie in Dex Juraperiode. Unter Den Pfflanzen Des Jeftlandes bezeicfnet Das 2 uftreten bon angiofpermen Difotyle= donen (Credneria [Ĩaf. I, Jig. 10]) in Der frreibezeit Den (5intritt einer neuten S̈tra Der Erogejobidte.

Tie flimatijchen 80 nen treten in Der freibezeit now biel beutlicher heroor, als in Jura; es laffen fich füblitife und nöroliche Bonen unterfcheiden. Wäfrent in ber nörd= lichen Bone eine Mifowutg Der ermähnten Tiermelt borwiegt, getwinnt in ber füblicken oder mediterranen Bone ein Mujchel= 
gefhlecht, bie Şippuriten (Taf. II, Jig. 18), eine foldhe Dber= Gand, Da $\tilde{\beta}$ alle anderen Formen Dagegen in Den Szintergrumb gedrängt werden.

(3) lieberung: Die Cinteilung bor Sreibe ift auf bie franzöjtifcten ßerbältniffie begrüntet, wo wir eine bolle Ent= witeflung fümtlicher (s)lieber itbereinander baben, während in Deutfichland Die einzelnen 2(bteilungen felten in gejhlofienem 8ufammenthang auftreten; Doch) lafjen fite fitch nicht foblor mit Den franzöjiffchen Normalverbältriffen in Einflang bringen.

Untere Rreide. Sie ijt gefennzeichnet burch einen all= mählichen übergang Der oberjuraffii don Tierwelt zu Der echten Sreidefauma. In Norbdeutichland fam es zu unterit zu brat=

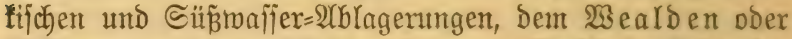

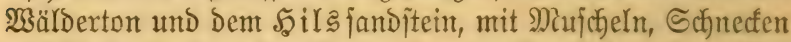

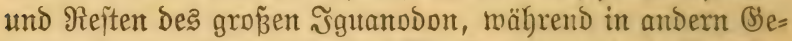

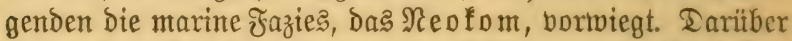
lagert ber Baalt, eine marine 2rblagerung mit grünen

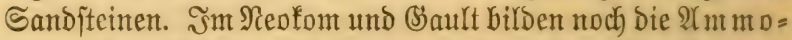
niten, jowohl bie gef hloffenen wie bie aufgerollten Formen, Den wichtigiten Teil ber Fauma. Unter Den Mufcheln find bie Inoceramus $=\mathfrak{2}$ rten von bejonderer $\mathfrak{B}$ edeutung (I. sulcatus) unto neben ihnen nod) Trigonien (Trigonia caudata).

Die obere 尺reibe mirb in Drei Brlieder eingeteilt: Das Senoman, Turon und Senon; im öitlicten Teutichland, mo bie Suaberfandjteine borkerrichen, jpricht man bon unterem, mittlerem und oberem Dutaber, zrif hen tweldhen zwei Miergel= zonen, Der untere und mittlere \$läner, Yicgen. Das Echtwer= gemidft der Fauna liegt mu in ben Seeigeln unv ben

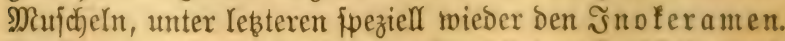

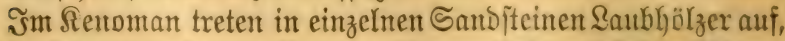
im Turon uno bor alfem im Senon finden fich bie Ylblage=

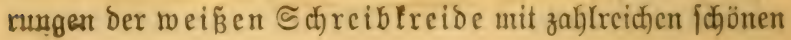


ßetrefaften, unter melcten Die Seeigel und Die Iekten Fornten Der Belemmiten fehr gute Seitfoffitle fino.

Wie fchon ermähnt, ijt die $\{$ ü oliche 3 one ganz verjocieden bon ber nörolicten; Dort herrjchen bie Mujcheln aus Dem (Sie= ichlechte Der Shamiden bor, in Der unteren Sreibe Durch Caprotina und Requienia bertreten, während in Der oberen

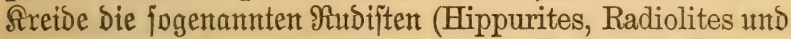
Sphaerulites) fo maffenhaft merden, dap̃ fie viele hunbert Metex mächtige 2 blagenungen exfüllen (Şippuritenfalfe).

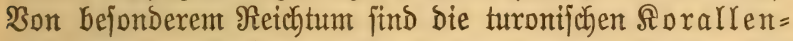
jacichten Dex (S) ojau, welche in Den Dortigen Bucbten zur Âtabildung famen und bon forallen, Mujweln und Schnecten exfitllt find.

\section{Viertes Zeitalter \\ oder die känozoilichen formationen.}

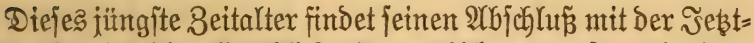
zeit und zeigt bie aflmähliche Szerausbilloung unjerer jesigen Tiex = und Pflanzentwelt, fowie Dex ganzen Erobberfläche aus Den Sejtalten, welche im mejozoijchen Beitalter zur (Entroick= lung famen. Fïr Den (Seologen fommen mux noch die (Ent= widflungsperioden felbjt in Betradyt, welche in die Tertiär= und Dilubialzeit fallen, während das fertige Bild des fäno= zoijoben Beitalters, die Šestzeit, bon Den Bologen, Botani= fern und (Seographen*) exforjdyt wiro.

In einex furzen Sharafteriftif find etwa folgende Momente gegenüber Dem mejozoijchen Beitalter geltend zu machen. $\mathfrak{A}$ if

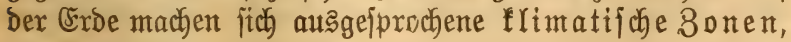

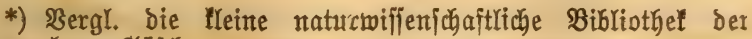

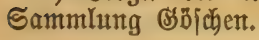


forwogl in Der \$flanzen = wie in Dex Tiermelt geltend. Die Pflanzen zeidyen fich Durch bie Entfaltung Der angio= ipernen Difotyledonen, dex ßaubhölzer, aus, gegen welche die Bahl Der (Sinnnofpermen einte berfarmindend fleite ijt. In Dex Tiermelt herrichen bor allem bie ऽäugetiere

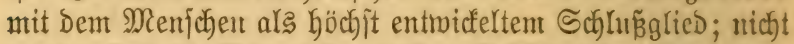

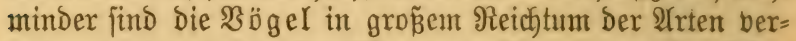

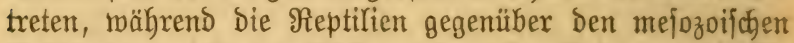
(S) Dechfen, Schlangen, Srotodile und Schildströten baben noch Bebeutung. Unter Den Antphibien find fröfdre und Sala= mandrinen an Stelle ber Stegofephalen getreten; bei ben

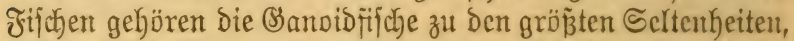

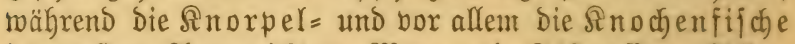

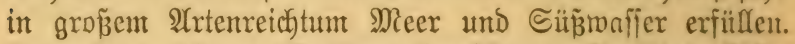
Unter den nieberen Geetieren herrichen nacte Tintenijiche, Mujcheln, Schneden, Seefterne und Seeigel, und die Siorallen werden Durch Die Entricflung Dex ßoriten und Mabreporen

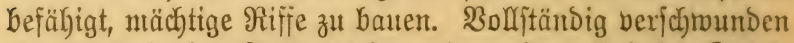
oder Doch in Der Tierwelt faum benterfbar find Die Siejel= fáfruämme, Seelilien, Brachiopoden und vor allem bie $\mathfrak{A}=$ ntoniten unto Belentniten.

\section{Die Tertiärformation.}

Das Tertiär ftellt, wie \{çon erwälyut, Den lübergang von Dem mejozoifchen Beitalter zur Эeßtzeit Dar, es ftelyt aber in

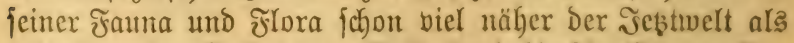

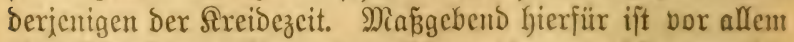
bie rafche Entroidfung Der Säugcticre, weldhe mun eine entfchcidende Siolle in Dex Ticrwelt fpielen, währeno in Der Pflanzentuclt dic Difoty)ledonen mefre und mehr die über= macht über Die übrigen \$Fflanzen gewinnen. YIteb in Der (S) $=$ ftaltung ber Erobberfläche geht eine llmmanolung und $\mathfrak{A n =}$ 


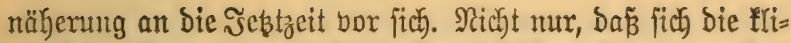

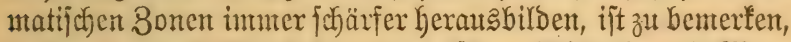
fondern es geitaltet jich aurd zmijifen Feitland und Mieer inmer mefr bas ßerfältnis, wie wir es jebt vor uns haben.

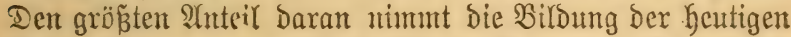

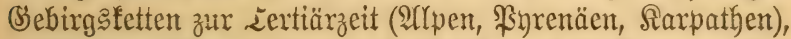
melche ztwar fochon früfer Durch Brïche und Docfernmgen Des Bodents vorbereitet maren, aber erjt zur Tertiärzeit ifre lebte und mäatige (Emporprefijuttg exfuthren. Saano in S2and mit ber Bildung ber teftonifichen (S) birge fant es auch zu nafjenthaften bulfanifichen Eruptionen. Die Enuptionsntafien bejtehen faupt= \{ächlicl) aus Bafalt, Bhonolith und $\mathfrak{A}$ nd efit= (s) citeinen, welche teils als majïge Sitppen und Doutbulfane, teils aber autch als echte Stratobulffane abgelagert find. Durch bie (Erfhebung Der (Sicbirge werben autch Die Strontgebiete reguliert und ber

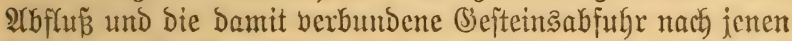
(Sebieter gelentt, welche auth heute noch biejem Bmect bienen.

Die 2rblagerungen Der Tertiärzeit finto natïrlic) yon ber berfobiebenjten $212 t$; anfangs treten audch noch auf ben heutigen Sontinenten marine 2 rblagenungen mit Salfen und ธant = iteinent auf, melche jich affmählich auf bie Nieberungen und

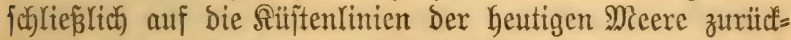
ziehent. Sie werben innerfyalb des Feftfantos bertreten burch

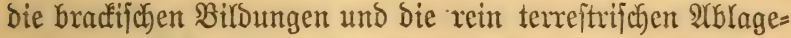
nungen, bejtehend aus den 2(bjäben bon Duellen, den $j o=$ genannten Süp̈wafierfarfen; Dieje find erfüllt bon Sumpf=und

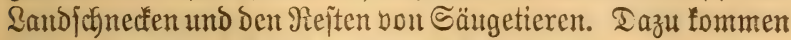

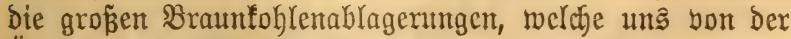
Üppigfeit Der F̌lora ßunde geber.

Die Schwicrigfeiten, welche fich einter allgcmeinen (S) liebe= rung bes Tertiärs entgegenfeßzen, find in bie $\mathfrak{A}$ ugen fpringent, ment wir an bie Beridjiebenartigteit ber 2 (blagenung felbjt, mie an bie Berfajiebentheit des filtimas Denfen, unter melchen 
Die betreffende Tier $=$ und Pflanzenmelt gelebt hat. (F) muß̈te

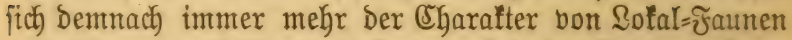
und = Floren außbilden, wie fie ja auch in ber Jebtrolt herr=

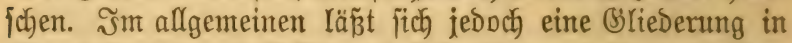
älteres Tertiär mit Cofofän uno Drigofän, und jüngeres Tex= tiär mit Miofän und \$riofän feititellen.

Die alten Tertiärgebilde E̊ofän und Dlig ofän zeigen in

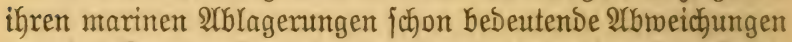
von Der Sireise. 2̂tmmoniten, Belemniten uno nahez̧ fämt= liche Brachiopoden find berichtounden, und an ifgre Stelle treten

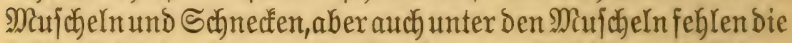

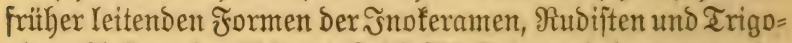

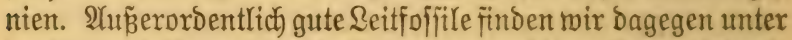

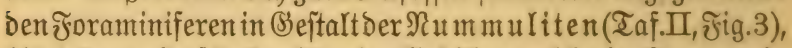
bis talergrope faheibenförmige (Bebilde, welche im snnern ein ipiral aufgeroltes Snjtem bon Sammern zeigen. Sn Den fït= lichen (ntebiterranen) STblagenungen fpielen bie Samumliten

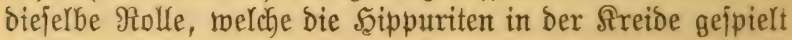
haben.

Nod) gröpere Bebeutung, bejonders in Deutfilland, ge= mintren bie terrejtrifcten Bildungen Des alten Tertiärs, welche

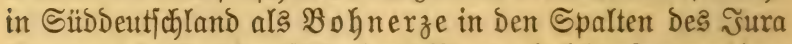

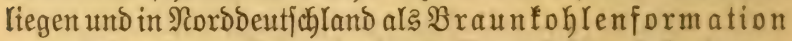
auझgebildet fint. Tie oligotänen Brauntohlen beherbergen eine Pflanzentwelt bon echt tropifdjem (E) a rafter mit Sequoia (Taf. I, ₹̛ig. 9), Palmacites, 2orbecr, Ficus, inmer= grïnen (Fichen ut. a.

Die Tiermelt miro beherricht bon Den Säugetieren, unter welchen Palaeotherium (Taf. IV, Fig. 9) uno Anoplo-

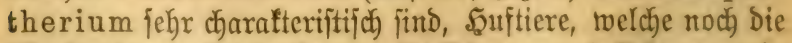

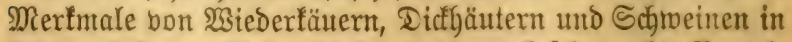

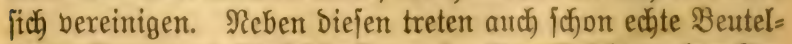

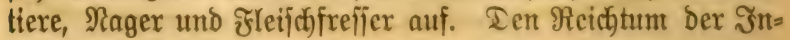




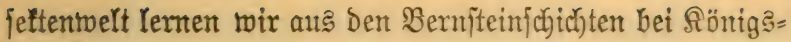
berg fenner.

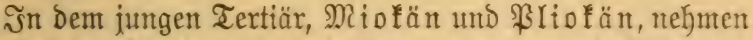
bie iebst Gerrichenden Ârten immer mebr zut. Die Riflanzen= welt bes Mriofäns in (Europa zeigt nod) einen fub tropif chen

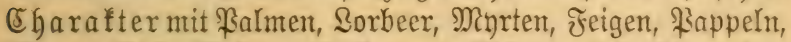

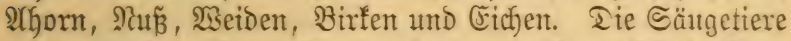
zeigen mentiger Sammeltupen, foubern nähern fich in igrer Form Den jeß̧t Yebenden; bie Dicffäuter find Durch Mas to don,

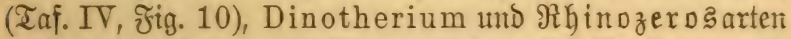
bertreten; unter Den Sguftieren zmeigen fitch SIntilopen und

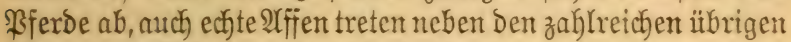
SăugetiergejahIcchtern ber Jeştzeit auf.

In Ieutfalland find zwet martine Arblagenungen bon be= fonderer Bedeutung, Die cine - Das Ma ainzer $B$ ecten in ben

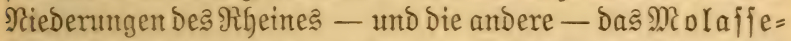
(5) ebiet am Plordrande Der $\mathfrak{A}$ (pen. - Während erfteres jich

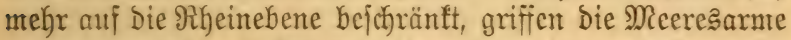
im Ionaugebiet zur Miofänzeit nod) tief indas Fejtland ein und jetlofien fith an bie Eeebecten an, weldje Den Nordrand ber Ârpen umgaben.

Die bulfanifáten (Eruptionen ber Tertiärzeit machten jich in Ientichlant befonders geltend; jie jint gefennzeidynet Durch Die B̉ajalte und \$Yhond litfe Der Sifel, Des Siebengebirges,

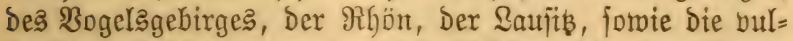

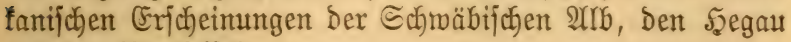

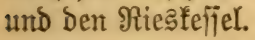

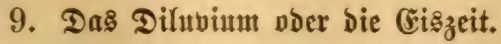

(Eine geologifoch noch feinesmega genügent aufgeflärte (Er $=$

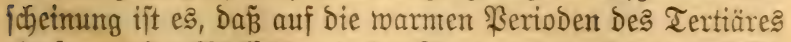

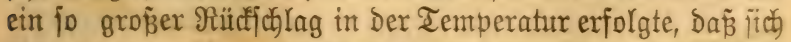

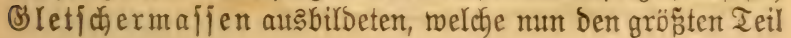


Der gentäß̈igten 8Dute bedectten. Jn ungeheuren Maffen wälzzte

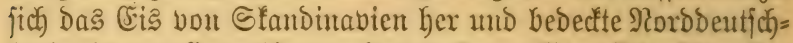

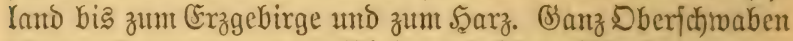
bis zur Tonau war vom アrbeintalgletfder, Dberbauern bon

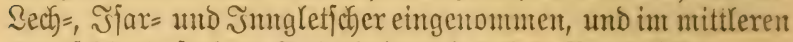

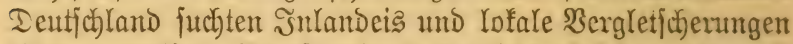

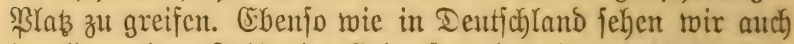
in alfen antern Teilen ber Eroe, fowohl in Den nörolichen wie fübliçen Szemifplyären, beutlicbe Spuren einer 2 (btitiblung bes frimas und Damit berbumbener meitgreifender $\mathfrak{B}$ erglet= idjerung.

Wo noch freies Sand üfbrig blieb, lebte bei uns eine nor= bijche Tierwelt; Das Manmut, cin langhaariger, nit unförm=

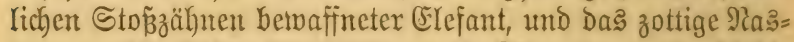
horn, nebit Şöhlenbär, Syläne und Söme waren wohl bie

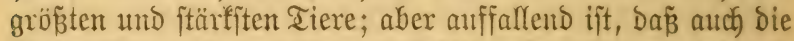
übrige Tiermelt cine überat? fräftige Entfaltung zeigt unt zum Teil zu gemaltiger (S) röß̄e Der Sfrten gelangt. Eo f̈nben wir in Curopa bie gropen Seirfche (Cervus giganteus) und Stiere (Bos priscus und primigenius) neben mohyentwicfelten Willopferden. SYlm auffallenditen ift bie Gröbzenentwieflung bei Den fübamerifantifchen (EDcntaten mit Deut Ricjenfaultier (Megatherium) unD Dent Riejengïrtcltier (Glsptodon). Mit

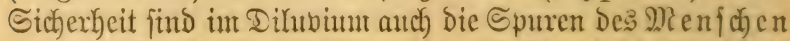

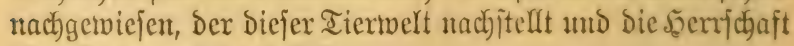
über fie zu getwinuen fucht.

Die (S) YicDerung Des D ilubiums ift ztwar nod) nicft in

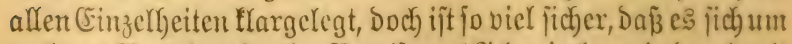

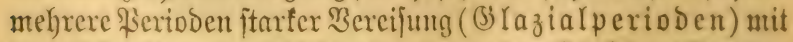

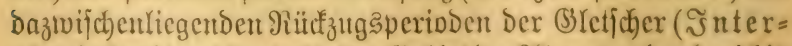
glazialzciten) fandelt. Jm (jebiet der? ?fpen werden drei bis vier, in Plorbdeutfed)land, England und Sfandinabien meift orci ßergletfonerungen angenoumen. 
(5) Garatterifiert find Die Yrblagenungen Dex Ciszeiten Durch die ungebeuren Majien von glazialem Schutt, welcher Durch

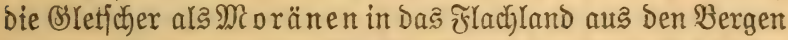

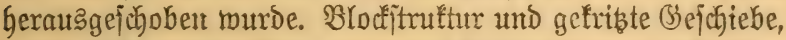

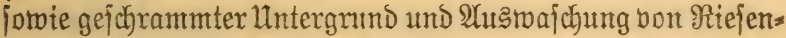
töpfen find Die Merfmale Diejer SYblagenungen. In Den İnter= glazialzeiten murben die Moränen ausgetwajchen uno in ben

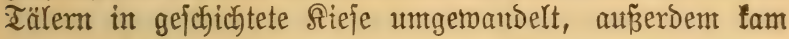

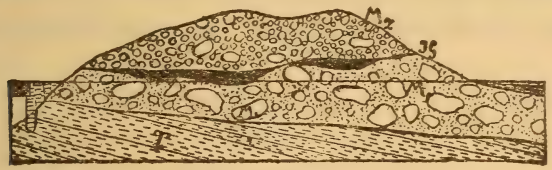

Diluvium.

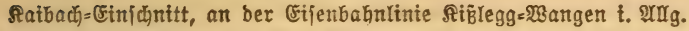

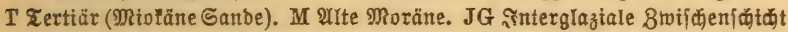

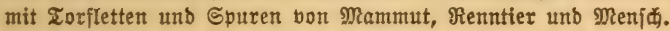
$\mathrm{M}_{2}$ รัunge Moräne.

eక్ auch zu 206lagenungen bon Toxf und Srauntohle. Das

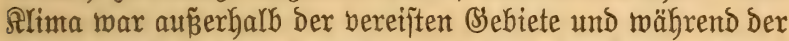
s̃nterglazialzeit bielfach trodéten und wird als Steppenflinta charafterifiert, in weldbem fich namentlich die mächtigen

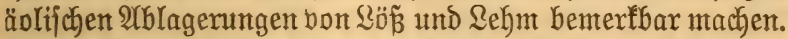
Die glazialen Arblagenungen find febr wichtig, Dent ihnen ber= banfen wix bielfach Die Fruct tbarfeit Des Bodent, und fie find es autch, welche unjere heutige Dberfläche und Talbildung be= einflunt Gaben.

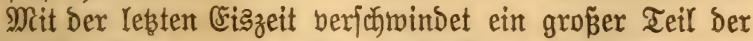
dilubialen Fauma, namentlich Die Diafläuter, und geht un=

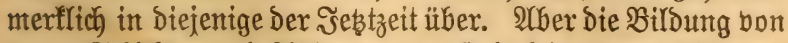
neuten Schichten und 2Yblagenungen fteht feinesmegs ftill, und man bezeichnet dieje mobernen Siebilde als 2 Iruvium. Szier= 
Ger gehören bie Suellabjäbe, bie Schotter= und Sandablage= rungen bes Meeres und ber flüffe und bor allem bie teil's vom Waffer, teils bom Winde gebildeten $\Omega$ ehm= und $\Omega o ̈ B=$ ablagerungen.

So jimb wir im rajchen frluge bon ben älteiten Ulrzeiten bis zur Jebztzeit Durct)georungen, von Der Şerrfchaft jener un=

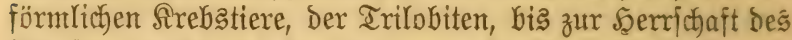
Menfchen, und haben Den Iangfamen aber fteten (Entricflungs: gang berfolgt, ber fitch burref Die ganze (Erogejecticte burch)= zieht und feinen (Sipfelpunft in Der J̃ektzeit und Dem Mienicten findet.

\section{Zurammenftellung der wichtigiten Pflanzen- und Cierveriteinerungen.}

2Uf Den folgenden bier Tafeln foll eine überficht über bas Bffanzen= und Tierleben gegeben werden. (Es ift natïrlich),

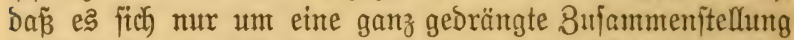

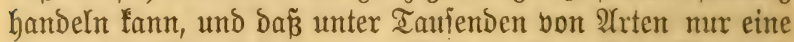
einzige als Inpus Der ganzen Sattung Kerautsgegriffen und abgebirbet merben fonnte. S̈mmerfin fömen wir auth'

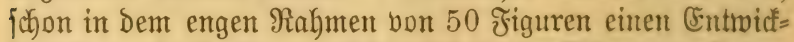
lungsgang beobacten, welcher fith in Der Bflanzenwelt bon

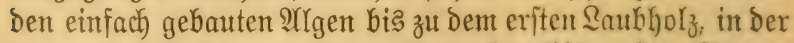
Tiermelt bon ben eimzelligen lltrierchen bis zu ben Säuge= tieren berfolgen läp̈t. Freilich ift bie Bufanmtenjtellung eine rein botanif(c) reip. zoologifche, robei bas geologijche $\mathfrak{A} u f=$

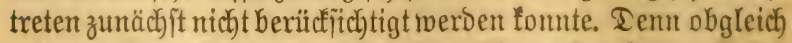

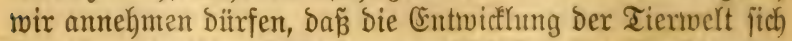
an bie geologif hen Perioben angefdyloffen hat, fo fint wir boct) noch) weit entfernt, bies an ber S.and von Foffitlien nact)= 
Bufammenftel. §. wiďtigft. FFlangen=u. Tierberfteinerungen. 95 meifen zu fönnen. Ter (B)rumb hierfür liegt zum Teil in ber noch immer fehr lüdfenfyaften Sientuti ber Beriteinerungen, Denn es find immerhin nur febr fleinte und im $\mathfrak{B}$ erbältnis zum Sianzer unbedeutende (Siebiete, melche bis jebt unterfuctst find ober überhaupt unterjucht merden fönnen, Da uns ja nux bie äน

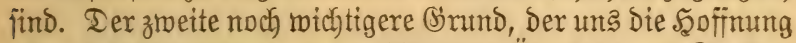

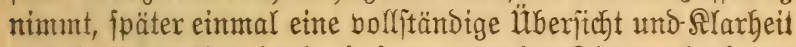
zu befontuten, Yiegt Darin, Dás uns von Den Tieren überfautpt

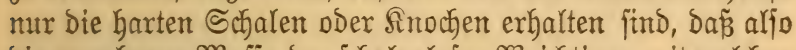

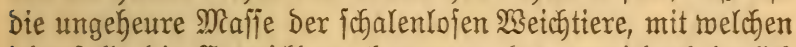
jedenfalls die Entwieflung begonnen hat, untwieberbringlich verloren gegangen iit. $\bigodot_{0}$ mitifien wir uns mit ben mentgen zum Teil fiur bie Entwicflungsgejchichte recht wertlojen STart= bejtandteilen begnitgen; um jo mehr ift bafür ber હcharfïin

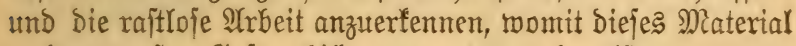

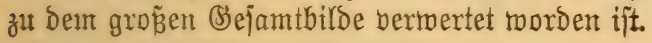




\section{Tafel I. Źofifle Pffanzen.}

Jig. 1. Chondrites ant Den నiasfichiefern bon Sirdjheim u. Tecf in Sürttemberg. Typus einer beriteinerten Meeres= alge, Die gleich Den heutigen Iangen maffentyafte (Entfaltung und $\mathfrak{B}$ erbreitung erreichte.

Fig. 2. Calamites. Şauptberbreitung in Der Steinfohlen= formation. Baumartige hochaufgefdifiene Schachtelhalme vom Baut Dex heute noch Yebenden Urten (ङ. 66).

Jig. 3. Sigillaria aus Der Steintohlenformation (rejtaut=

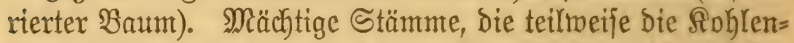
flöze zujammenjezen. Die injtematifche Stellung ift unjicher (ङ. 66).

テ̛ig. 4. Lepidodendron auక Der Stcinfohlenformation.

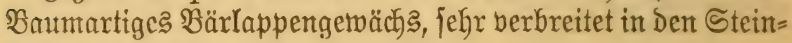
foblen (\$. 66).

F̛̀g. 5. Annularia auฐ Der Steintohlenformation. Blätter, welche zu Den Salamiten gehören (ऽ. 66).

テ̛ig. 6. Odontopteris.

Fig. 7. Sph enopteris. Beibe $\mathfrak{B l a ̈ t t e r ~ v o n ~ f o f f i t l e n ~ F a r n e n , ~}$ welche in Der Cteintoblen = und Iriasperiobe eine mäcbtige Entfaltung und Fonmenfüllle befommen.

Fig. 8. Zamites oder Pterophyllum. Bertreter eiter 3ytabeengattung Der Trias= und Șraperiode (ธ. 74).

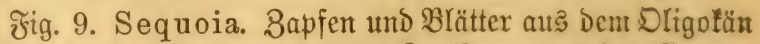
ber ๔rbmeiz. Bertreter fofïller Roniferen aus Der Brntple ber Taxuanarten.

Fig. 10. Credneria ats Dem Senomanjanditein bon Blanfenturg in Saarz. Dieje Blätter, welche in Die Srnuppe bon Ficus (Urticinae) geitellt werben, bezeict)nen in Deutich) Iand Das erfte IUftreten Dex ミaubhölzer (؟. 85). 


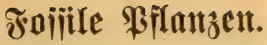
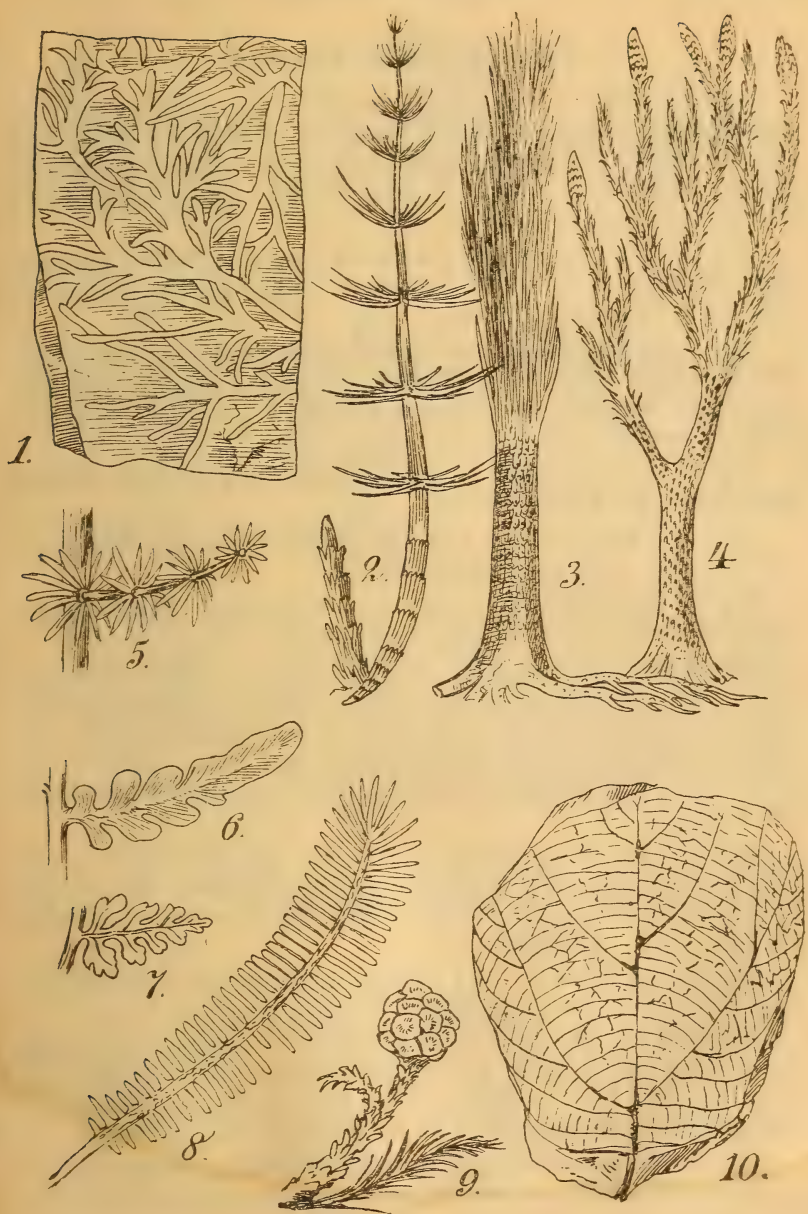

₹raas, હేeorogie. 


\section{Tafel II. J̊pifile Tierwelt.}

శ̧ig. 1-3. Foraminiferen, einzellige Urtiere (Protozoa) mit gefammerten Schalen. J̛tg. 1 Fusulina, Reitioffil im Rohlenfalt (ङ.64). Jig. 2 Globigerina, maffentyaft in ber weipen ভdjreibfreibe

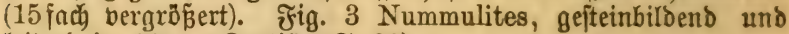
leitent im älteren Tertiär (S. 90).

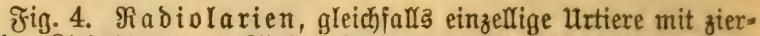
lichen Siejelffeletten. Sind bejonders wichtig für bie freueritein= billoung (175 fach vergröß̄ert).

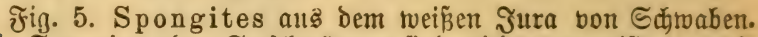
Die ভpongien ober Seejchtoümme find nieder organifierte, aufgemachiente Seetiere; in Der Эura= und Ireidezeit treten fie häufig gefteinbildent auf (ङ. 82).

Fig. 6. Halysites aus bem Dberfilur bon S5otland, Iqpus einer tabulaten Roralle (S. 57).

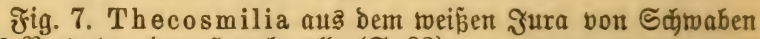
als Bertreter einer ફ̧eraforalle (ভ. 83).

Jig. 8. Braptolithen aus bem böhmifhen Silur, wahr= icheinlich zu den Şyoroiopolypen gehörig (ङ. 60).

frig. 9-11. Rrinoiben oder Seelilien, Strabltiere mit Iangem Stiel, einem §elch und $\mathscr{A}$ rmen. Fig. 9 volfitändiger Encrinus liliiformis aus bem Mulduelfalf von Erailsheim (ङ. 73).

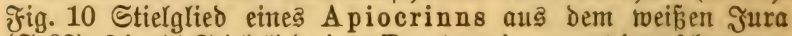

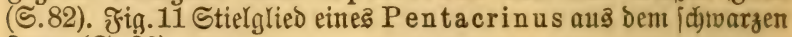
Эิura (ङ. 80).

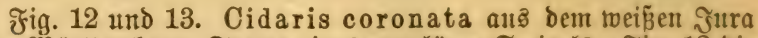
von $\mathfrak{W}$ ürttemberg. Iypus eines regulären Seeigels. Ffig. 12 die vollfändige Sdyale ohne Stadgeln. Fig. 13 ein einzelner Stadel (ङ. 82).

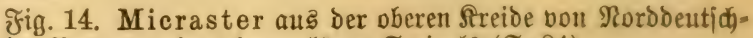
lanto. Bertreter eines irregulären Seeigelä (5. 84).

Fig. 15-17. B̧radiopoden oder $\mathfrak{A r m f i e m e r , ~ T i c r e ~ a u s ~}$ ber (S)uppe Der $\mathfrak{B u ̈ r m e r ~ m i t ~ n u t j d e l a ̈ h n l i c h e n ~ E đ a l e n . ~ F i g . ~} 15$ Spirifer aus Dem Debon Der Eifel; Jig. 16 Terebratula aus

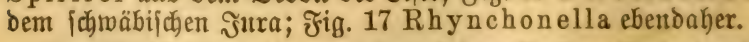

Fig. 18. Hippurites aus ber alpinen ภreide. Bertreter einer gänzlich auşgeftorbenen frembartigen (s) mip pe bon Miujcheln (S.86). 


\section{Foijile Iiermelt.}

\section{Tafel II.}
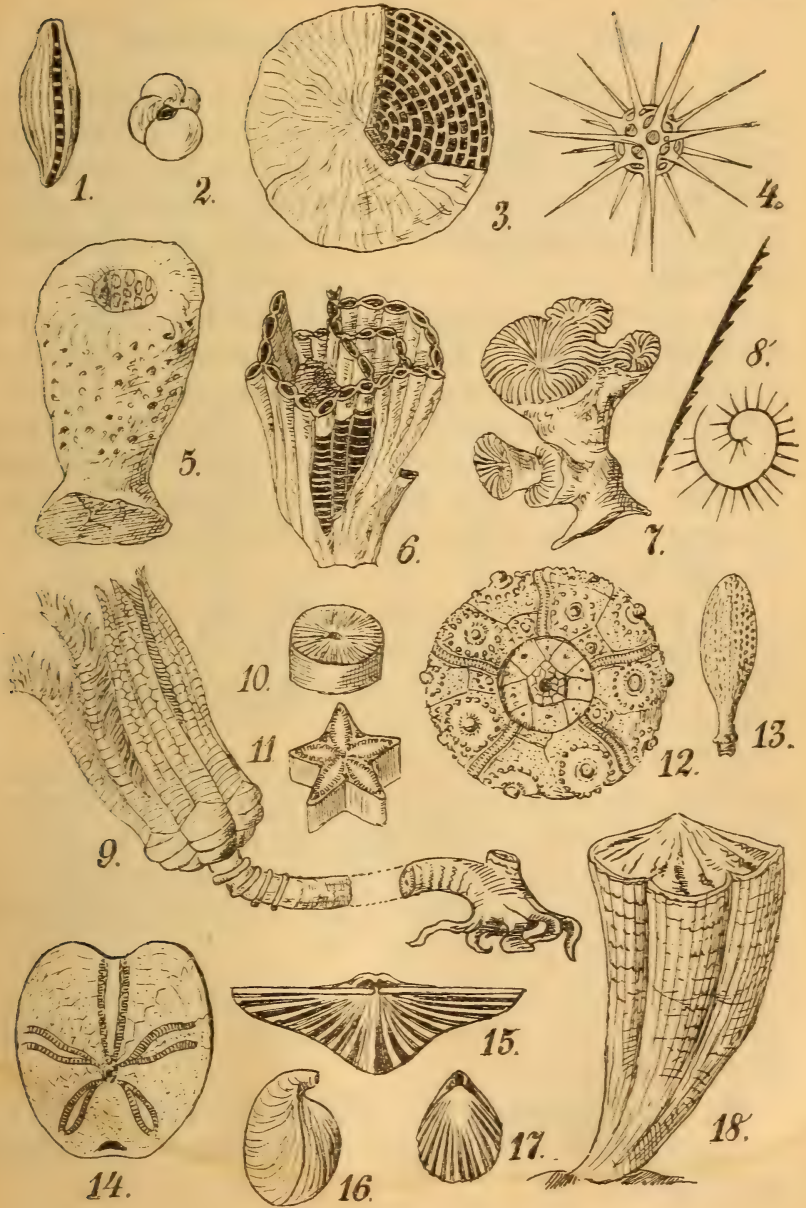


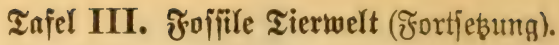

Fig. 1-3. Nautiliden, fchalentragcude Sopff̈̈̈̈lex oder Rephalopoben. Fig. 1 Orthoceras aus Dem હilux bon Böhnten (\$. 60). Fig. 2 Cyrtoceras aus Dem Iebon Der

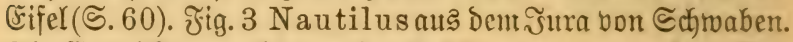
Die Sntwidflung geht bon ber ftabförmigen zux aufgerolten Fornt ïber.

Fig. 4-7. 2lum onitiben, auझgejtorbene Simppe Der Rephalopoben. Fig. 4 Goniatites ant Dem Dberbebon der Fifel (ङ. 63), Beginm Der Ammonitenreike. Fig. 5 A mmo-

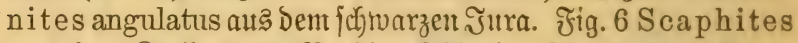
aus der Rreide bon श्रordbentichland. Fig. 7 Baculites ebenbaber (S. 84). Tie Entrodflung beginnt mit ben ge= f(d) loffenten Formen und endigt mit Den offenen und ftab= förntigen Slrten.

Fig. 8 แnd 9. Belentniten (S. 79). Fig. 8 rejtauriertes Belemnitentier. Fig. 9 Belemnites aus dem (d)trä=

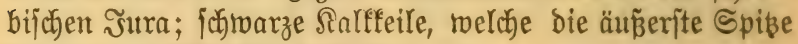
des interen Sfelettes baritcllen.

ङig. 10 und 11. Trilobiten, auţgeitorbene Sruppe der

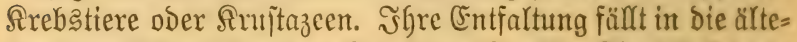
ften petrefaftenfitbrenden (chichten (ङ. 60). Fig. 10 Paradoxites bohemicus anț Den Rambrium bon Böhmen. Fig. 11 Phacops latifrons aut Deut Iebon Der Fifel, aufgeroltes Tier mit groß̄em Jacettenauge.

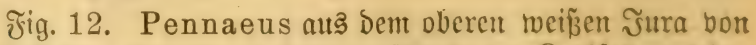

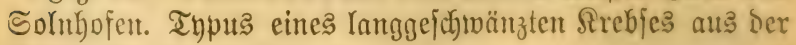
Sruppe der Srameelen.

Fig. 13. Cancer (Psammocarcinus) ants Dem Tertiär bon Frantreich). S:in Bertreter Der furzgej(d)ränzten Sirebje oder §rabben. 
Fojiile Tierwelt (Fontję̧ung).

Tafer III.
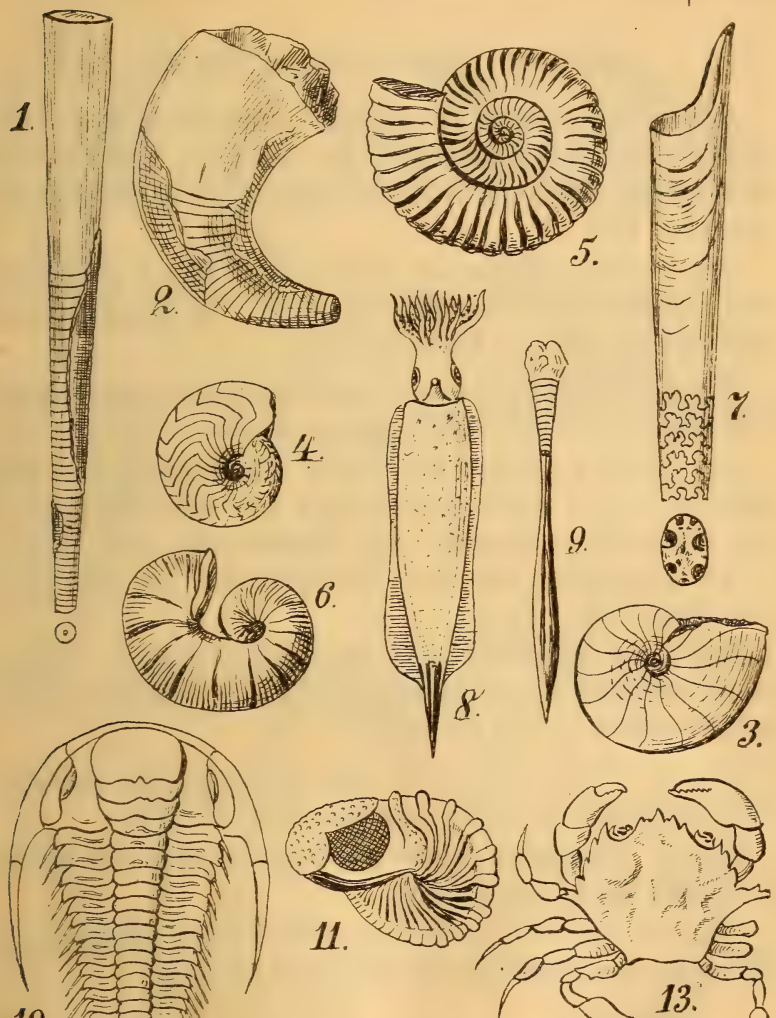

10
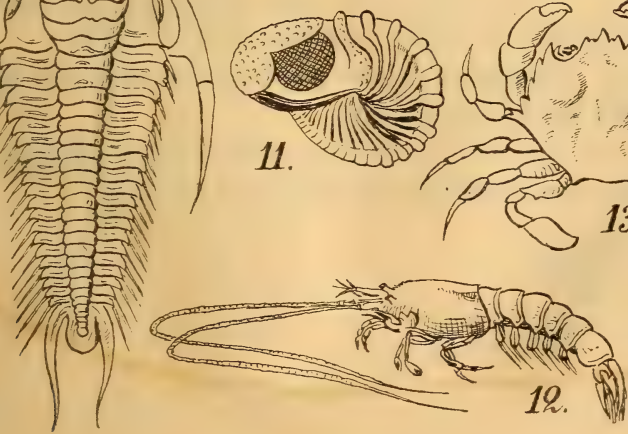


\section{Tafel IV. ช̛ofifile Tierwelt (Sd)lü).}

3ig. 1. Pterichthys aus bem "alten roten Sandftein" (Dberbebon) bon Scjottland; ein Bertreter ber feltjamen Panzerfif che, mit welchen bie (Entwidflung ber Wirbeltiere be= ginnt (S. 64).

Fig. 2 und 3. 3ähne bon 5 aifif

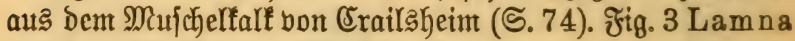
aus Dem Miofän von Dberjchtwaben.

Fig. 4. Semionotus, ein Schmelzfdyuppen= Dder Stanoid = fijch aus bent Seuper von Stuttgart (S. 74).

Fig. 5 und 6. Stegofephalen, bie Borläufer ber $\mathfrak{A m}=$

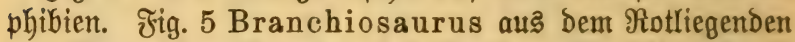
Des ßlautenfchen (Brundes bet Dresben (S. 69). ซ̌ig. 6 Trematosaurus aub bem Buntianditein bon Bernburg a. b. Saale (ธ. 71).

Fig. 7. Ichthyosaurus ober Fifchfaurier; pracht= volles mit ber S2att erfaltenes Eremplax aus dem oberen Lias von ßirctyeim u. Teff (ธ. 80).

f̧ig. 8. Pterodactylus oder FIngfaurier aus ben litfographif chen Schieferm bon Solntlofen (S. 78).

J̛ig. 9. Palaeotherium aus dem Dligofän von ßaris; Borläufer der $\mathfrak{P f e r b e ~ ( \Im . ~ 9 0 ) . ~}$

Fig. 10. Mastodon aus Dem Miofän bon Steinfyeim in Wirttentberg; Borläufer Der Elefanten (ভ. 91). 


\section{Fojiille Tiermelt (ভূhluñ). Tafel IV.}

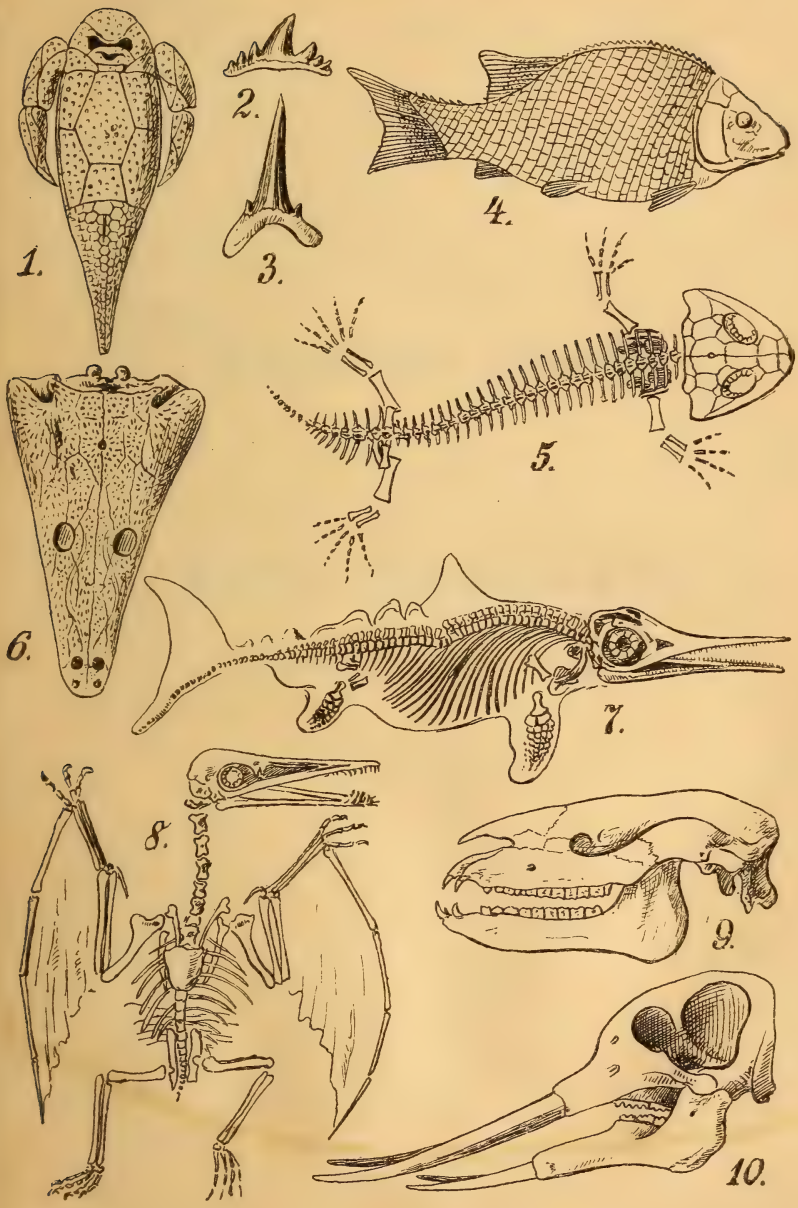





\section{Iabellarijade 1tberjitut}

über Die

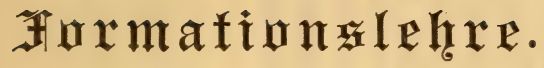


\$iftorijøe Sseologie.

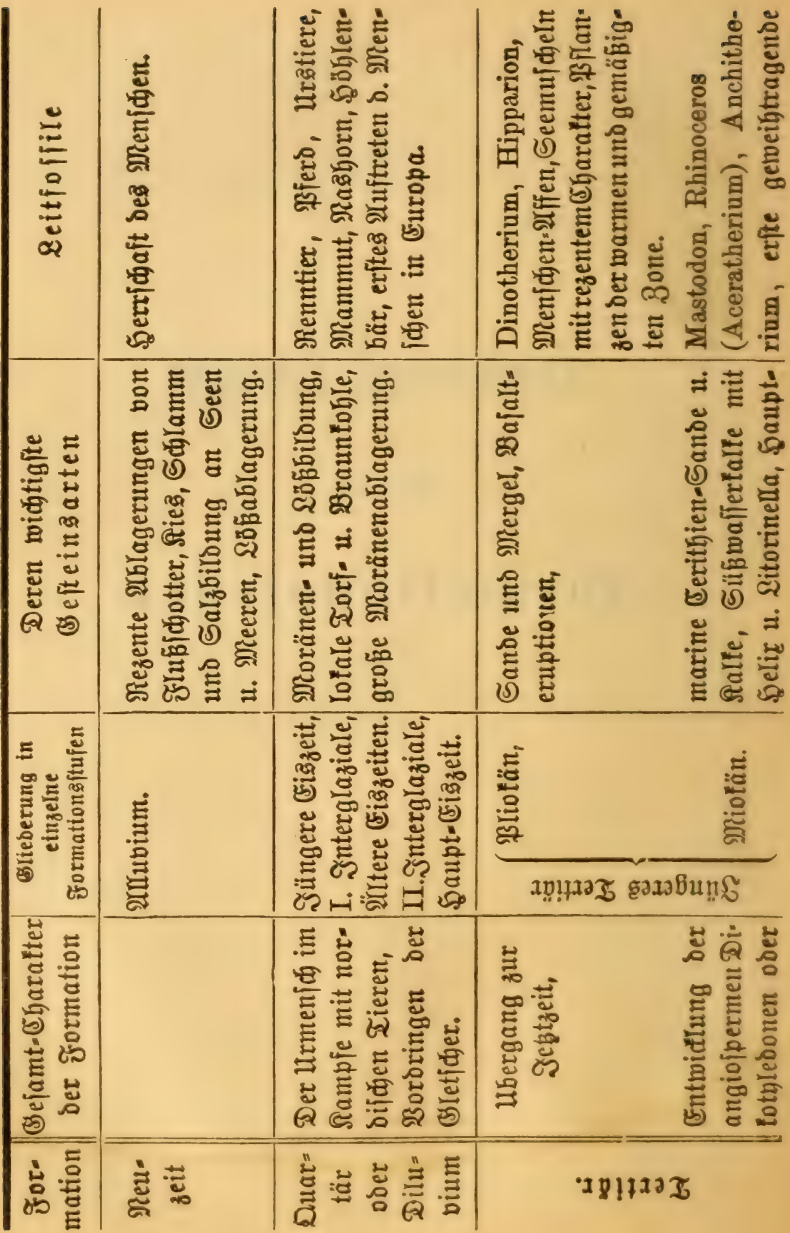


Tabellari|dye überficht über bie Frormationßlegre. 107

\begin{tabular}{|c|c|c|}
\hline 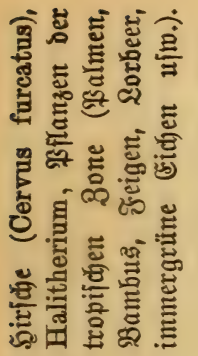 & 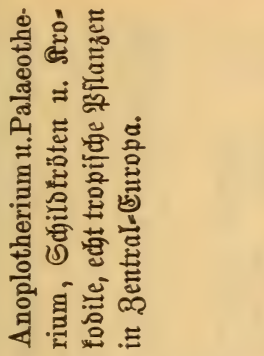 & 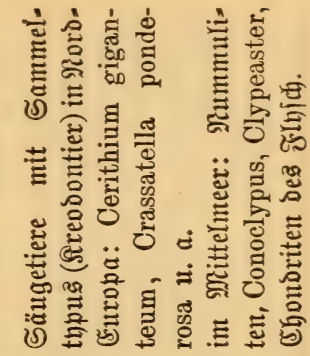 \\
\hline 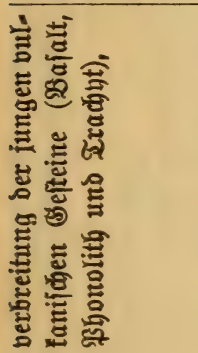 & 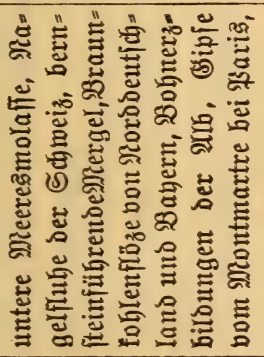 & 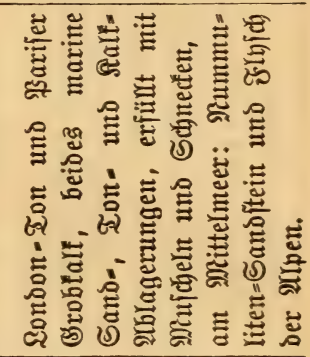 \\
\hline
\end{tabular}
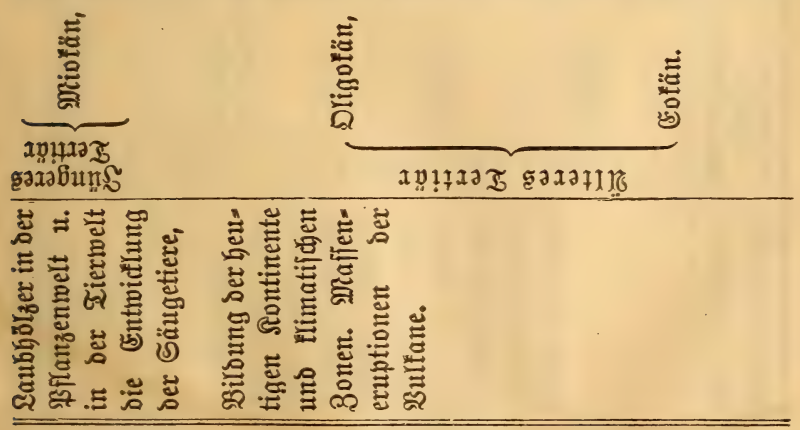


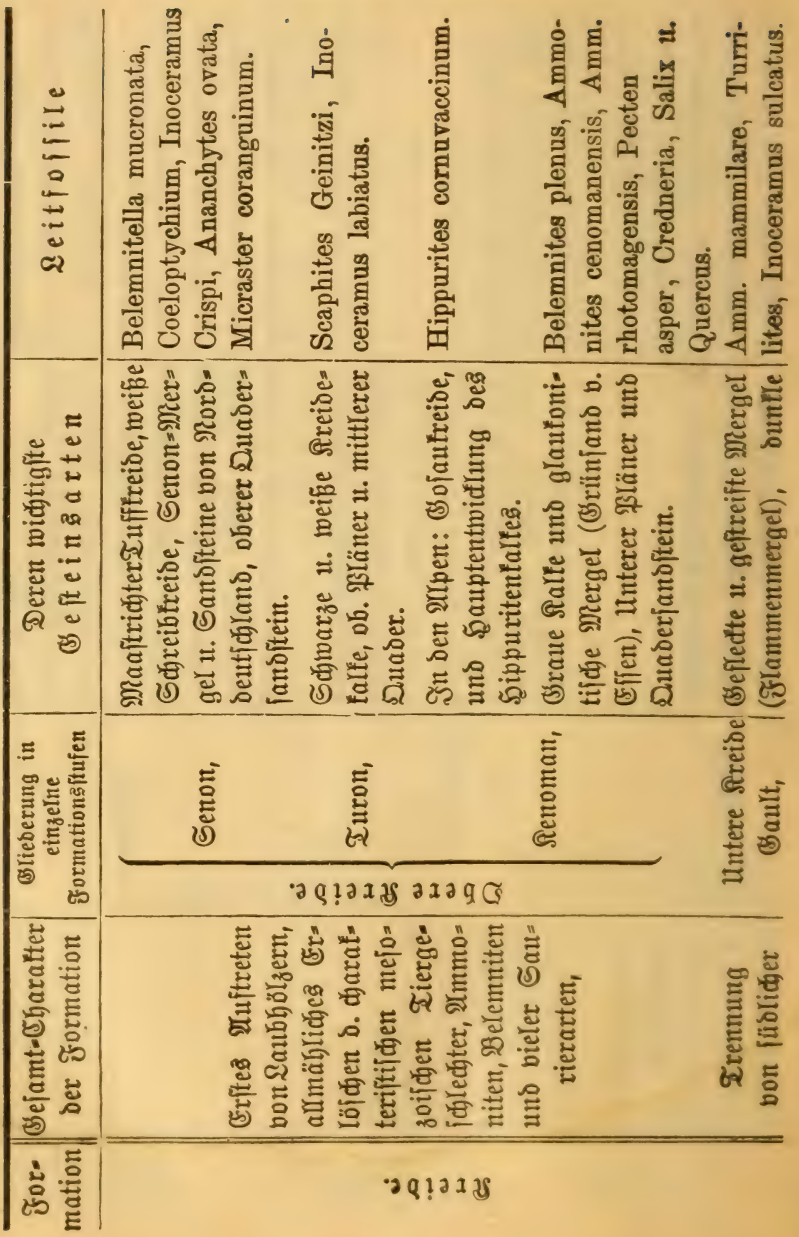




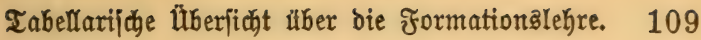

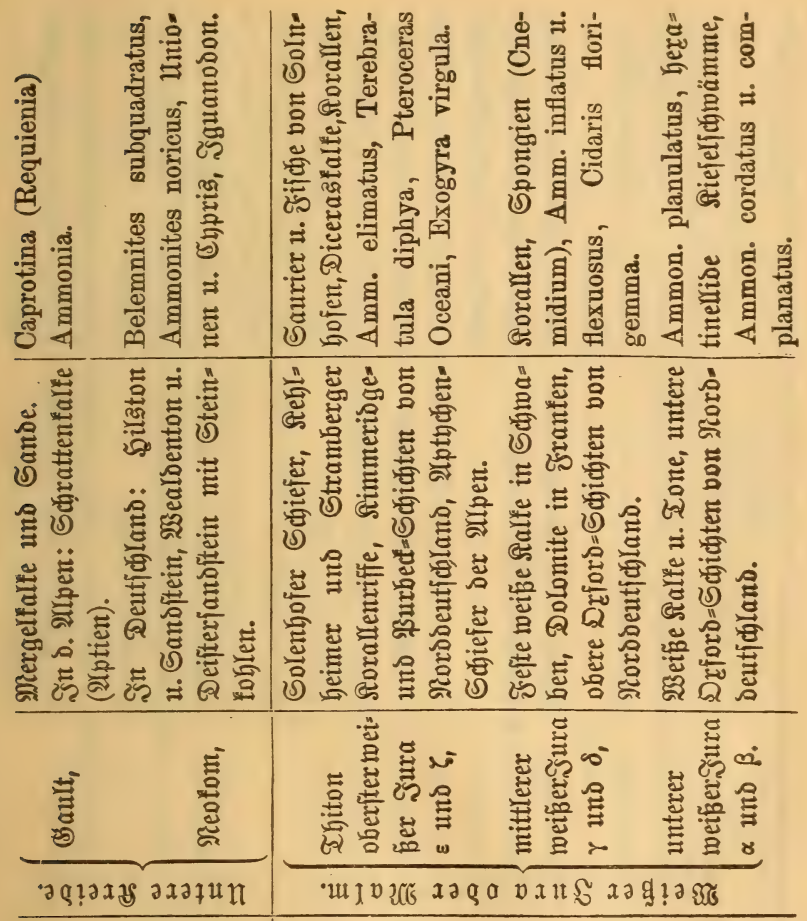

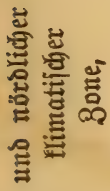




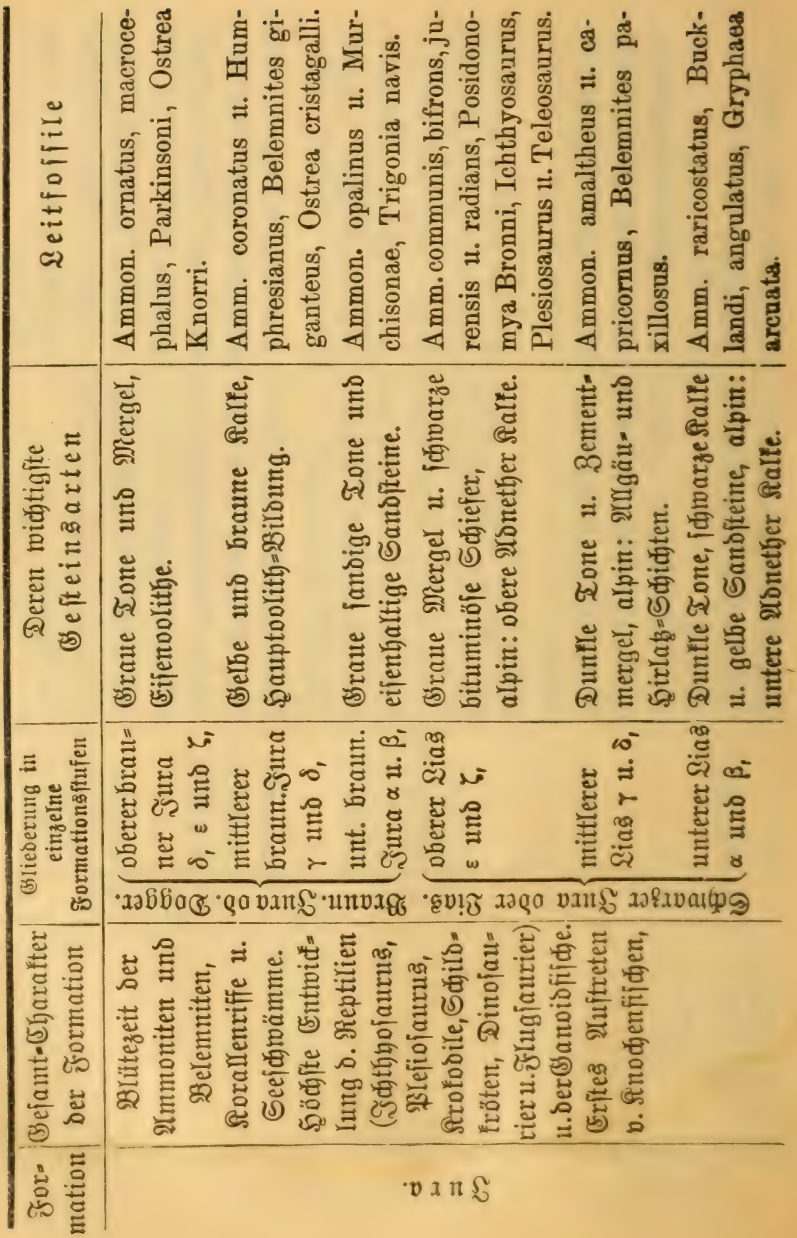




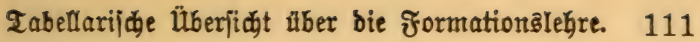

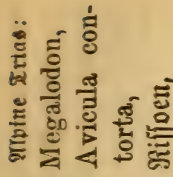

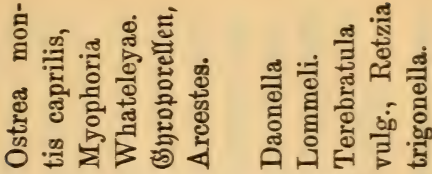

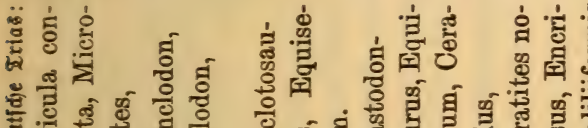

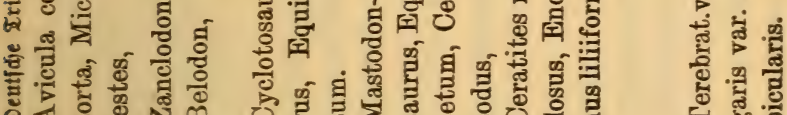

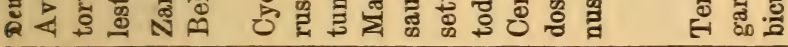

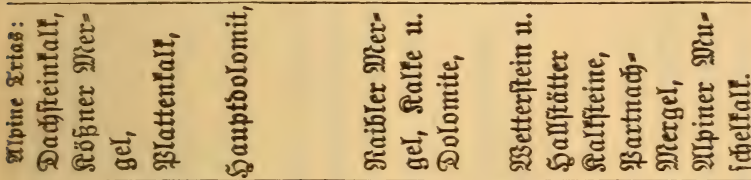

\begin{tabular}{|c|c|c|c|}
\hline 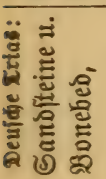 & 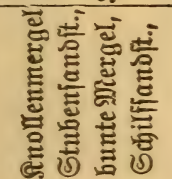 & 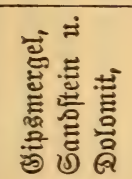 & 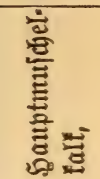 \\
\hline
\end{tabular}

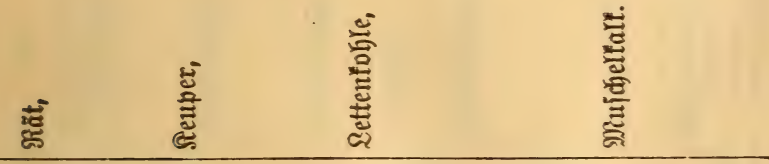

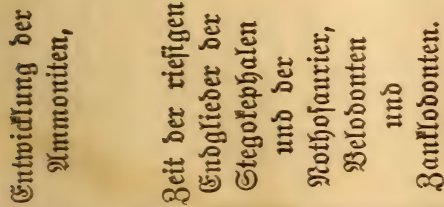

• $\mathcal{E} \mathfrak{I} \mathfrak{I}$ 


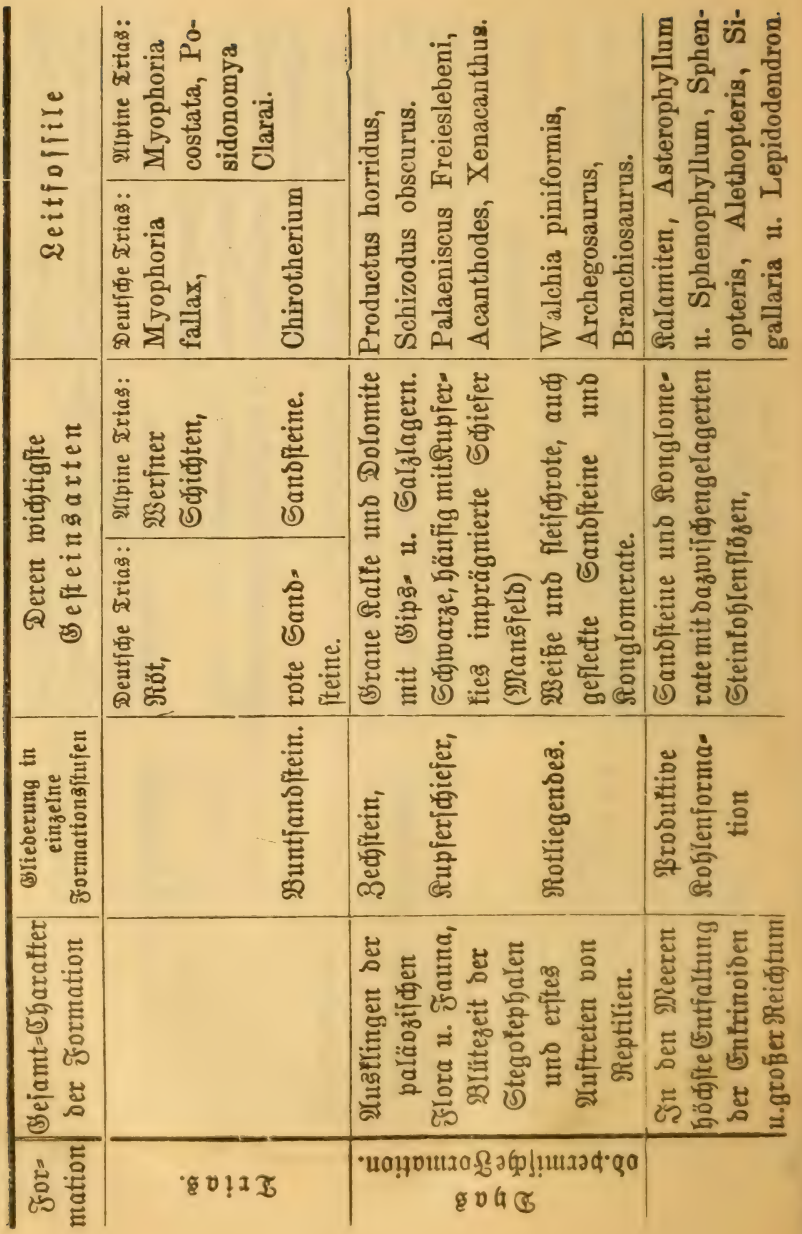




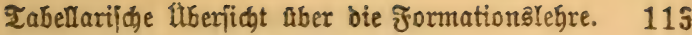

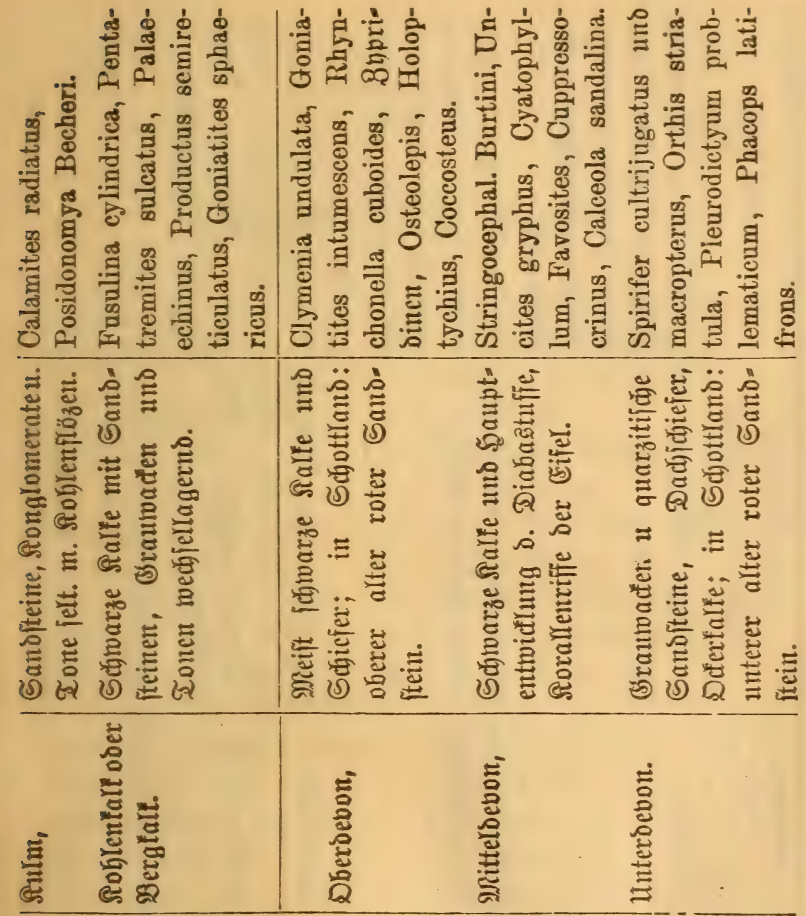

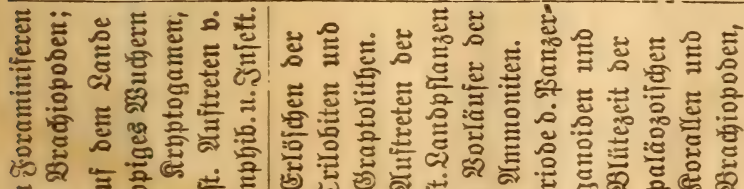

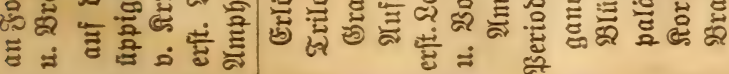

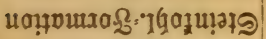

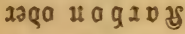

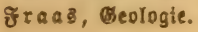




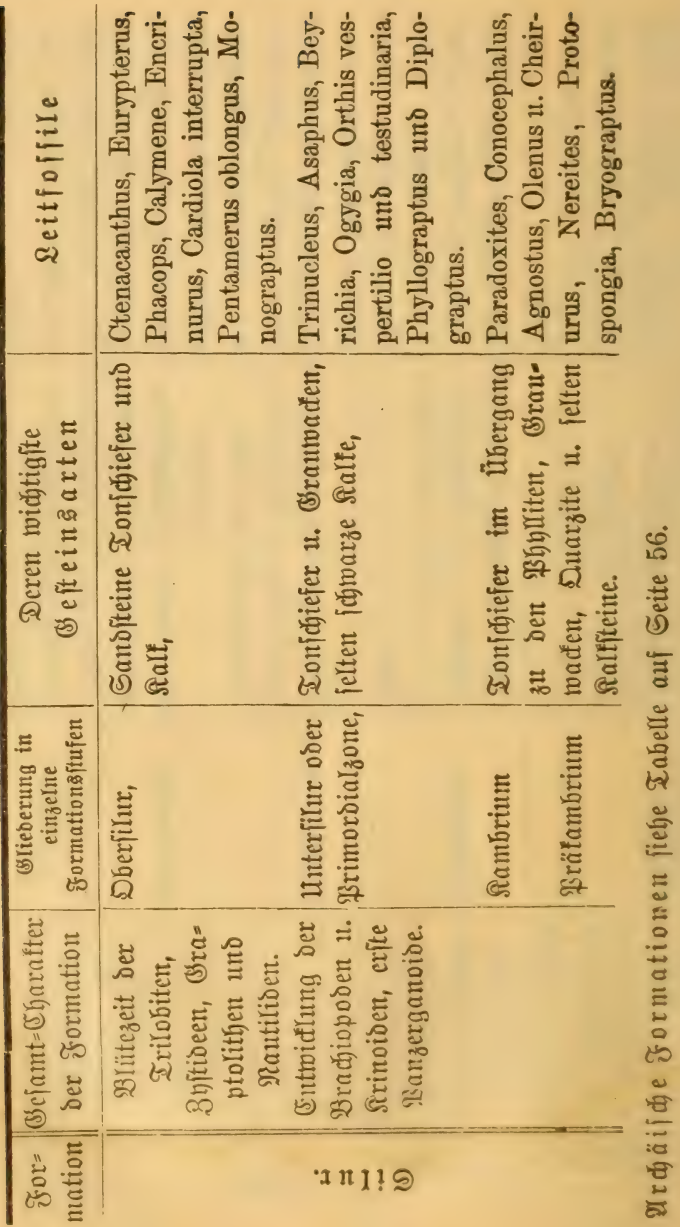




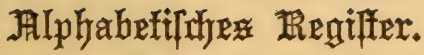

Acrodus 74.

Aëtosaurus 74 .

affen 91.

Agnostus 60 .

थ̈goferaten 79.

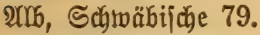

Alethopteris 66.

श्रीuvium 93.

2rlpine Trias 75.

2lmaltheen 79.

2 mmoniten 63.64 .70 .73 .75 . 78. 79. 80. 81. 82. 83. 84. 86 . Ammonites angulatus 100 . Ôtmmontitioen 100.

2Tmphiboljđjiefer 20. 54 .

Andefitgeíteine 89.

Antyndrit 15. 47.

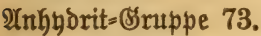

Annularia 66. 96.

Anoplotherium 90.

Anttgrazit 16. 17.

Ipatit 15.

Apiocrinus 82. 98.

2raufarien 66.

Archaeopterys 78 .
Irrçäij呫e Frormationen 53.

Archegosaurus 69.

Irrieten 79.

2rofoje 21.

Artefificher $\mathfrak{B r u t n t e n} 48$.

Asaphus 60.

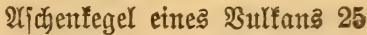

2ripiboferaten 83.

Asterophyllum 66.

Atlantosaurus 78.

Urugitjdjiefer 16.

2luttern 79.

Avicula 75. 77.

azoiju 56.

Baculites 84. 100.

Barranco 26.

Bajalt 19. 28. 29. 30. 89.

Belemniten 70. 78. 79. 81. 84.

87.

Belodon 74. 75.

Bermiteinjodichten 91.

Bocca 24.

Bofnerz 90.

Bontebed 75.

Bos priscus $\mathfrak{u}$. primigerius 92 . 
Bradiopoden 57. 60. 61. 63. 64. 69. 73. 75. 85. 98.

Branchiosaurus 69. 102.

Brauneifenitein 13.

Braunfohle 16. 17. 31. 89. 90. 93.

Breccie 22.

Burudgebirge 44.

Buntjandjtein 71. 76.

Burgandftein 75 .

Calamites 66. 96.

Calceola 62.

Cancer (Psammocarcinus) 100.

Caprotina 87.

Ceratites 73.

Ceratodus 74 .

Cervus giganteus 92 .

Chirotherium 71.

êhloriogefteine 13.

Ebloritidicejer 16.

Chondrites 96.

Cidaris 82. 98.

Coccosteus 64 .

Credneria 85. 96.

Crioceras 84.

Cupressocrinus 63.

Cyathophyllum 62.

Cyclotosaurus 74 .

Cyrtoceras 60, 100.

Dacŭjidutefer 59.

Devon 61.

Diabaa 18. 29. 30. 58.

Diadema 82.
Diageneje 35.

Diatomeen 38.

Diluvium 91.

Dinofaurier 74. 78. 85.

Dinotherium 91.

Diorit 18. 28. 29. 30. 58.

Dogger 81.

Dolomit 15. 47. 76. 83.

Dombulfane 27.

Dyas 68.

Echinoconus 85.

שॄॅintodermen 39. 82. 84

Echinus 82.

EFflogit 20.54.

Einfache Befteine 11.

Einiturzbeben 46. 47.

달 35.

Eisberg 36.

Eifenerze 13.

Eişgefteine 12.

ธָiszeit 36. 91.

ETemente 11.

Encrinus 73. 98.

ㄷofär 90.

Eozoon 55.

Equifeten 74. 75.

Erobcber 45.

Eroperiode 51.

Erofion 48.

Eruptibgefteine 10. 18

Ẽaltengebirge 43.

Farnfrönter 66.68 .

ชażę 51. 
Fenerftein 12.

Fijogfaurier 102.

Fłexูu| 82.

Flugiaurier 78. 85. 102.

Foraminiferen 39. 64. 84. 90. 98.

శormation 51.

Frittung 31.

Futotben 59.

于rumarolen 31.

Fusulina 64. 98.

(3)abbro 18. 30.

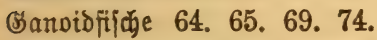
78. 80.

(5)ault 86.

(s)ebirgabilloung 42.

ङefäp̈ fryptogamen 58. 61.

Šeijer 32.

Semengte Bsepteine 11. 18.

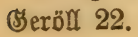

(3eidichtete Bsefteine 20.

Seichidatete $\mathfrak{B u l f a n e} 26$.

Seichiebe 22.

(B)ếteinaైarietäten 10.

Gigantostraca 61.

달 15. 47. 73.

(3ipşmergel 75.

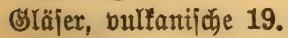

(STaufonitfande 84.

Blaziale 8eit 36. 92.

Brletider 35.

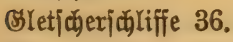

હlimmeriđiefer 20. 31. 54 . 56.
Globigerina 98.

Glyptodon 92.

ઉ̈neis 20. 54. 56.

Sontiatiten 63. 100.

Srabenverjentung 44.

Granit 18. 28. 30. 58.

ङ大ranulit 20. 54.

Sraptolithen 60.98.

Errautwaden 59.61. 65.

Briffelichiefer 59.

ङ̋ruttomoräßte 35.

Grrünjande 84.

জrrünittein 18

Gryphaea 79.

5aifitidge 65. 74. 85. 102

Şalltätter §aff 76.

Halysites 98.

5̧arpoleraten 80.81 .

Şarze 16.

Şilsjandittein 86.

Sippuritenfalfe 87.

Hippurites 86. 87. 98.

5öhlen 47.

ફ̋őhไenbär 92.

5̧orizonte 53.

5ernblendeidjiejer 16.

Sornftein 12.

Sorite 44.

Hybodus 74. 102.

Iabeit 16.

ฐapipis 12.

Ichthyosaurus 80, 85. 102.

Iguanodon 85. 
Inflaten 83.

Эnlandeis 36.

Inoceramus 86.

Snietten 67.78 .

Эnterglazalal=Zeit 92.

Suraformation 77.

Salamarien 66. 68.

Ralfipat 14.

Salfftein 14. 31. 38. 47. 59.

61. 70. 76. 79. 83. 89.

Ralftuff 15.

Sambrium 59.

Ranal eines $\mathfrak{B u l t a n s} 25$.

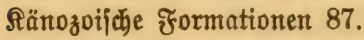

Raolin 21.

Rarbonate 14. 47.

Rarbon=Frormation 64 .

Rentoman 86.

Rephalopoden 60. 63. 64. 100.

Reuper 73.

Tiez 22.

Riejelgejteine 12.

Riejelgur 13. 38.

Riejelichiefer 12.

Siejelfinter 13.

Rlaftifche Bsefteine 11. 21.

Rlimat. 8onen 77. 85. 87.

SIlymenien 63.

Snodgenfifiche 70. 85. 88.

Siohlen 16. 38.

Roghtenbeden 66 .

Rohlengefteine 16.

Sobjenfalt 64 .
Rohlentwafier|toffe 16.

Ronglomerat 21. 65.

Soniferen 69.

Sontaftmetamorphoje 31.

Rontraltion Der Erorinde 40.

Rorallenriffe 42. 62.

Sorallentiere 39. 57. 61. 62 .

64. 75. 78. 81. 83. 84. 87. 88.

Sraterrand 26.

Sreibe 15. 39.

Sreideformation 83.

Srintibert 39. 57. 61. 63. 73 98.

Sriftallinijüe Schiefer 20. 34

54. 56.

Srofobile 78. 85.

Sultm= Formation 65 .

Supferichiefer 68.

Sabyrinthodonten 74 .

Raffolithe 28. 30.

Lamma 102.

Iaba 25.

Reitfoffite 53.

Lepidodendron 66. 96.

Settenfohle 75.

¿ías 79.

Lingula 60.

Lituites 60.

ฉoี

Entoferaten 80.

Miaare 28.

Magneteijerftein 13.

Mainzer Befđen 91. 
Malm 82.

Mammut 92.

Manganterze 13.

Marmor 14. 31.

Mafitge Sefiteine 18.

Mafifige Bulfane 27.

Mastodon 91. 102.

Mastodonsaurus 74.

Meerjaurier 78. 79. 80. 85.

Megalodon 63.

Megalosaurus 85.

Megatherium 92.

Melanerpeton 69.

Melapyyr 19. 30. 58.

Menichen, exfte 92.

Mergel 21. 75. 76 79. 86.

Mejozoijaje Formationen 69.

Metamorphoje 35

Metopias 74.

Micraster 84. 98.

Microlestes 75.

Mineralgänge 47.

Mineralquellen 47.

Miofän 91.

Mittelmoräne 36.

Mofetten 31.

Molaffe 91.

Moränen 35.93.

Mosasaurus 85.

Murchisonae 81.

Muidelfalf 72.76.

Meuidelttiere 39. 63.65. 75. 78.

79 80. 81. 85. 86. 87. 88. 98.
Mitopforien 73.

Mashorn 92.

ఇauttiliden 57. 60, 61, 63, 64. 100.

Nautilus 100.

Neofom 86.

Reovulfantijage Bejteine 30.

Nereiten 59.

Neuropteris 66.

Nitratgefteine 13.

Nothosaurus 73. 74.

ఇtımmuliten 90. 98.

Obolus 60.

Odontopteris 66. 96.

Dodontornithen 85.

Dligofän 90 .

Dolithbildoungen 81.

Dppelien 82.

Orthis 60.64 .

Orthoceras 60. 100.

Ditreen 81.

Palaeohatteria 69.

Palaeoniscus 69.

Faläontologie 52.

Palaeotherium 90. 102.

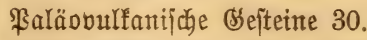

ßałäozoif che Formationen 56

Palmacites 90.

Paradoxites 60. 100.

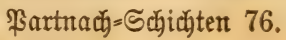

Pecopteris 66.

Pennaeus 100.

Pentacrinus 80. 82. 98. 
Beripghintten 82.

ßerm 68.

Phacops 63. 100.

\$honolity 19, 28, 29, 30. 89.

\$hosphate 15.

\$ho $p$ horit 15.

Pygllit 20. 31. 55. 56.

Phylloleraten 80.

Fhytogene Sefteine 38.

\$lätrer 86.

\$lanulaten 82.

Plesiosaurus 80. 85.

Pleurodictyum 62.

Pleurotomaria 63.

ßliofän 91.

Pliosaurus 85.

\$lutonifhe Sefteine 30.

\$olieridiejer 13.

Posidonomya 80.

ßräfambrium 59.

Productus 69.

Proterosaurus 69.

Pteranodon 85.

Pteraspis 61.

Pterichthys 64. 102.

Pterinea 63.

Pterodactylus 78. 102.

Pterophyllum 74. 96.

Euaderjanditcin 84. 86.

Quarz 12.

Duarzit 12.

Duarzporpgyr 18. 30. 58. 68.

Quellbil bungen 48.
Sucllen, heiß̄e 32.

Diabiolarien 39. 84. 98.

Radiolites 87.

গiaibler Schichten 76.

Rätijabe Stufe 75, 77.

Iiautbruade 15.

Reptilien 69. 70. 85.

Requienia 87.

Rhamphorhynchus 78.

rifitozeros 91.

Rhynchocephalia 78 .

Rhynchonella 79. 82. 85. 98.

Siogenfteine 15. 81.

Miöt 72.

gioteijenftcin 13.

Rotliegendez 68.

giudiften 87.

Eäfulare $\mathfrak{x e b u n g e n ~ u n d ~ G e n t - ~}$ tunger 41.

Ealbänder 29.

Ealpeter 14.

Salze 13.

Salzițđäe 68. 73.

Sand 22. 84 .

Sandjteine 21. 31. 59.61. 63.

65. 70. 75. 84. 89.

Säugetiere, erite 75 .

Säưenabjonoerung 29.

Saurier 73. 74. 78. 79. 80. 85.

Scaphites 84. 100.

Echadhtelfyalme 66. 74.

Edillofröten 78. 85.

Edilifintoftein 75 . 
Sallammbulfane 32.

Sđhneden 63. 65.75. 78. 79. 85. 88.

Shrubibtreide 84.86.

Schutt, bulfaniider 21 .

Schroammtall 82.

Sedimentär=-Šejteine 10.20.33.

Seeigel 57. 70. 78. 82. 84. 86. 88. 98.

Seetilien 39. 57. 61. 64. 78. 80. 82. 98.

Seejめ)พämme 39. 78. 82. 98. Seefterne 57. 78. 82. 88.

Seitenmoränen 36.

Semionotus 74. 102.

Sennn 86.

Sequoia 90. 96.

Serpentin 16.

Sigillaria 66. 96.

Silifate 16. 47.

Silux 60.

Solfataren 31.

Solnhofer Sdgiefer 83.

Somma, Monte 23.

Spatangus 84.

Spateijenjteir 13.

Spedfitein 16.

Sphaerulites 87.

Sphenopteris 96 .

Spirifer 60. 62. 98.

Spongien 39. 82. 84. 98.

Springquellen 32 .

Staffeibrum 44.
Stegofephałen 67. 69. 70. 71. 74. 102.

Steintogle 16. 17. 31.

Steinfohlenformation 64.

Steinjalz 13. 47. 68.

Stephanoferaten 80. 81.

Straflitiere 39.

Stratobulfane 26.

Stringocephalus 63.

Stuberianditein 75 .

Sulfate 15.

Süß̄paffierfalfe 89.

Enenit 18. 30. 58.

¿afelbrüche 44.

Ialfichiejer 16.

Teftonifide Erobeben 45.

Teleosaurus 80 .

Teleostei 70 .

Terebratula 73. 79. 82. 83. 85 98.

Tertiärformation 88 .

Thecosmilia 83. 98.

Titanerze 13.

Tityon 83.

Toneijemitein 13.

Tongeiteine 21. 47. 70. 76.79.

Toniçiefer 59. 61.

Topfitein 16.

Torf 16. 93.

Tractyt 19. 30.

Tranģgreffition 42. 77.

Trematosaurus 71. 102.

Triaš-formation 71 . 
Triglyphus 75.

Trigonia 73. 81. 86.

Trilobiten 57.60. 63. 64. 100.

Trocjitenfalf 73.

Trümmergefteine 11. 21.

Tuffe 21. 23. 25. 30. 38.

Turon 86.

Turrilites 84 .

iitbergangągebirge 55 .

Uncites 63.

Urtonidaiefer 20. 55.

2erwerfungslinien 44.

Bertwitteruttg 47.

Вејu⿰ 22.

Bögel, erite 78.

Voltzia 71.

Bulfanifche Grobeben 45.
B̧ulfanifde Bebirge 45

ญulfaniżmus 22.

2Gajīertätigfeit 34. 46.

TSealden 86.

Wellentgebirge 73.

Metterjteinfalf 76.

Wind 36.

Zamites 96.

Zanclodon 74.

Bechjtein 68.

Beitalter 53.

Zoantharia rugosa utto tabulata 57. 61. 98.

8oogene Befteine 38.

8)fabeen 74 .

8y)́tideen 61. 


\section{KONRAD GRETHLEIN'S VERLAG IN LEIPZIG}

In unserm Verlag erschien:

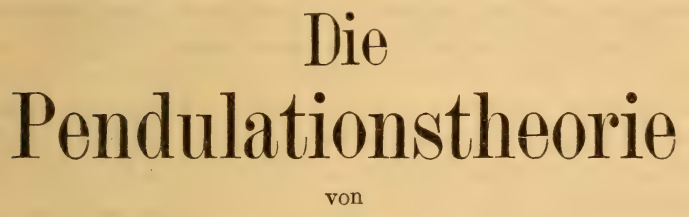

Dr. Heinrich Simroth

Professor an der Universität Leipzig

36 Bogen, Lex. $8^{\circ}$, mit 27 teils zweifarbigen Karten Preis: Broschiert $12 \mathrm{M}$, in Halbfranz geb. $14 \mathrm{M}$.

V

or einigen Jahren stellte der Ingenieur P. Reibisch in zwei Vorträgen vor dem Verein für Erdkunde in Dresden die Pendulationstheorie auf, die er mit einer Anzahl Tatsachen aus der Geologie und Biologie begründete. Der Verfasser obigen Werkes wurde alsbald ron ihrer Tragweite gepackt und suchte sie in einer breiten Skizze ,ttber die wahre Bedeutung der Erde in der Biologie“ (Ostwalds Ann. der Philosophie) einem größeren Publikum zu verdeutlichen. Verschiedene Aufsätze und Vorträge vor der Deutschen Zoologischen Gesellschaft u. a. a. O. behandelten seither dasselbe Thema, blieben aber teils zu sehr zerstreut, teils nur auf einzelne Kapitel der Biologie beschränkt, so daß die abgerissenen Publikationen höchstens als Stichproben gelten konnten. Die $Z$ wischenzeit wurde nun benutzt, um womöglich das gesamte Material der Zoologie, einschließlich des Menschen und seiner Kultur, immer in Verbindung mit der Paläontologie, die wichtigsten Daten der Botanik und der Geologie zu prüfen, inwieweit sie vor der Theorie bestehen. Alle Gebiete liefern glänzende Bestätigungen, eine Reihe von Tatsachen bleibt gleichgültig, keine einzige aber scheint in Opposition zu stehen. 
Die Theorie besagt zunächst, daß die Erde zwei feste Pole hat, Ecuador und Sumatra, zwischen denen die Nordsüdachse langsam hin und her pendelt. Die Pendelausschläge bedeuten die geologischen Perioden; in der diluvialen sowohl wie in der permischen Eiszeit lagen wir weiter nördlich, in der Kreide und im Eozün weiter südlich. Dadurch, daß die einzelnen Punkte der Erdoberfläche, am stärksten unter dem Schwingungskreis, d.h. dem Meridian, der durch die Beringsstraße geht und von den Schwingpolen gleichweit entfernt ist, unter immer andere Breite rücken und damit ihre Stellung zur Sonne und ihr Klima verändern, wird die ganze Schöpfungsgeschichte auf ein kosmisches Prinzip zurückgeführt. Der Unterschied zwischen dem großen und dem kleinen Erdradius (zirka $22 \mathrm{~km}$ ) hat dabei eine wesentliche Folge. Das flüssige Wasser nimmt jederzeit die Form des Rotationsellipsoides ein, das durch die Zentrifugalkraft bedingt wird. Da die feste Erdkruste erst allmählich in der Gestaltänderung folgen kann, ergeben sich abwechselndes Auf- und Untertauchen der Küsten, Trockenlegen und Verschwinden von Landbrücken. Der Wechsel zwischen Land und Wasser enthält aber den stärksten Anreiz für die Weiterbildung der Lebewesen (neben der Anderung des Kilimas). So kommt es, da B unsere atlantisch-indische oder afrikanisch-europäische Erdhälfte, und hier wieder unser zerrissenes Europa, der Ort ist, auf dem die ganze Schöpfung zu ihrer jetzigen Höhe heranreifte. Wie hier die menschliche Kultur sich entwickelt hat, so ist hier der Mensch entstanden, so vor ihm alle Lebewesen, soweit sie sich in der Paläontologie rückwärts verfolgen lassen. Von hier aus haben sie sich in bestimmten Linien über die ganze Erde verbreitet, so daß selbst Erscheinungen wie der Wanderzug der Vögel zu mathematischen Problemen werden und ihre Erlkärung finclen. Die geologischen Perioden und Formationen, der Vullkanismus, die Erdbeben, selbst die meteorischen Erscheinungen der Atmosphüre folgen denselben Linien. Die ganze Schöpfung wird folgerecht und kontinuierlich. Ja die astronomische Ursache der Pendulation, der Aufsturz eines zweiten Mondes in Afrika, scheint durch die neuesten Spekulationen englischer Astronomen bereits der Sphäre des rein Hypothetischen entrückt zu sein. 


\section{Auszüge aus einigen Kritiken über Simroth, Die Pendulationstheorie}

Die gesamte wissenschaftliche Presse hat sich, zum größten Teil in langen Artikeln, mit dem Werke beschäftigt.

„Der Globus": ... Es ist ein auBerordentliches Verdienst Simroths, erkannt zu haben, welcher Anwendungsmöglichkeit diese ursprünglich ja rein geologische Theorie für die Welt der Organisation fähig ist. Die Gesetze der Verbreitung, die uns bisher nur zum kleinsten Teile greifbar waren, rücken durch ihn in ein helles Licht und werden mit einem Schlage zugänglich und verständlich. Ja man darf ruhig sagen: der größte Teil der verschlungenen Fäden des Schöpfungsplanes ist entwirrt und läßt sich nunmehr übersehen. ...Wir widerstehen der naheliegenden Versuchung, weitere Stichproben aus dem wichtigen Werke zu geben; bei der Fülle interessanten Materials weiß man in der Tat nicht, wohin man zuerst greifen soll! Unser Urteil über das Buch glauben wir nicht präziser zusammenfașsen zu können, als wenn wir es für eine wissenschaft. liche Tat erklären! Ja wir sind überzeugt, daßes in ähn. licher Weise befruchtend und anregend a uf die gesamten biologischen Wissenschaften wirken wird, wie einstmals Darwins unsterbliche Schöpfung selbst.

,Neue Weltanschauung" ${ }^{6}$ : ...Die Wissenschaften, be. sonders Astronomie, Geologie und Biologie, werden sich noch lange und viel mit dieser Theorie befassen müssen, die, wenn sie sich als richtig herausstellen sollte, in vielen Punkten einen Umschwung in fundamentalen Fragen der Entwicklungslehre herbeiführen würde.

„Deutsche Revues6: ... Das Studium von Simroths Pendulationstheorie ist von höchstem Interesse. Die Reichhaltigkeit der Belege erinnert an Darwins berühmtes Werk, das ja gerade seinen zahlreichen Beispielen den Sieg verdankte. $\mathrm{Zu}$ wünschen wäre nur, daß sich auch die Geologie und Astronomie mit dieser Theorie be. schäftigten, denn diese beiden Wissenschaften sind es, die über ihre Berechtigung oder Nichtberechtigung zu entscheiden haben.

,Mitteilungen über die Vogelwelt": ...A A us Simroths kost: barem Buch, dem ich eine ebenso große kommende Be- 
deutung beimesse wie den Darwinschen Werken, kann ich weitere ornithologische Anzeichen einer wieder. kehrenden ,Tertiärzeit" herauslesen...

„Deutsche Entomologische Zeitschrift": ...Eine kiihne Theorie, geistreich a usgebaut! Eine neue Biogeographie nach den starren Gesetzen des Pendels und der Sonnenstellung... Ich kann die Pendulationstheorie zwar nicht für die endgültige Lösung der Schöpfungsfrage halten; ihr hoher Wert liegt für mich aber in ihrem heuristischen Prinzip; sie reizt zu neuem Forschen an, rüttelt alte $Z$ weifel wieder auf, stellt übersehene Fehler fest, kurzum fordert zur Kritik heraus. Gern betone ich jedoch zum Schluß, daß ich dem überaus anregenden Buche auch große positive Verdienste zuspreche; die ungeheure Masse des bewältigten Materials imponiert nicht nur, sie bringt a uch jedem riel Neues! Bisher begnügten sich oft die Zoogeographen damit, die Kolonisationswege festzulegen. Simroth vertieft ron neuem die Fragestellung dahin, weshalb die Lebewesen gewandert sind, er rertieft sie auf Grund eines geradezu phänomenalen Wissens!...

Die ,Zeitschrift für Mineralogie, Geologie und Paläontologie“ bringt eine sich über vier Nummern erstreckende, 21 Seiten füllende, zustimmende Besprechung.

,Zeitschrift für die österreichischen Gymnasien" am Schluß einer 10 Seiten langen Besprechung: ... Auch wenn man von der Pendulationstheorie absieht, so sind die Ergebnisse der Simrothschen Forschungen für die Biogeographie von hohem Werte und sichern dem Werke in unserer naturwissen. schaftlichen Literatur einen Ehrenplatz.

,Zeitschrift für Schulgeographie": Die Prinzipien der Pendulationstheorie, wie sie Paul Reibisch festlegte, biologisch zu stiitzen, ist der zugegebene Grundzug dieses mit enormem Wissen und Fleiß geschriebenen Buches. Jedenfalls ist die Pendulationstheoric ein ausgezeichnetes Mittel zu einer Systematik der Biogregraphie geworden, ihr Wert als Arbeitshypothese ist cin nicht zu mutcsehätzender, so daß dieser Versuch nicht nur für den Tier- unct Pflanzengeographen, sondern auch dem Geographen überhaupt durch das riesige Detailmaterial von Wert sein diurte.

Wissenschaftliche Beilage der ..leipziger Zeitung": Wir können dem großzügig angelegten Werk, das selbst die meteorologischen Erscheinumgen der Atmosphäre mit in den Bereich der Theorie zieht, das vor allem jeglichu Zukunftsspekulution beiseite lïßt, nur die weiteste Verbreitung wiincelen. 
Gegründet 1833. Dr. S. Frant3, Bonn a. Rb. Rbeinifches mineralien - Fontor.

fabrik und Verlag mineralogijaber und geologijober Lebrmittel.

\section{Geologifch-paläontologifche Lebrmittel}

für ben Schulgebraud):

\section{Allgemeine Beologie.}

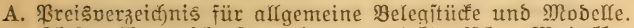

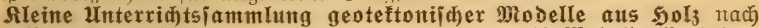
ßrof. E. Sialfowstn in Dresden:

12 Modelfe iN. $110 .-$ Gammlung von 4 Miodellent aus 5 ol3 3ur Berdeutlidung Der Dagerung und Berteilung Der Sd)idhtgeiteine in geitörtent (5egenden nad) $\mathfrak{B r o f}$. Dr. J. Malther: Boridule Der Geologie. III. Utữlage: MR. 32.Einfader Ipparat zur शad)ahmung Der widjtigiten Sdidtentörungen rach) Brof. Dr. I. $\mathfrak{B}$ alther:

פ⿹. 16.-

Geognoitifie Reliefs folgender Bebirge: Sarz, Saijerjtufl, elletict)=

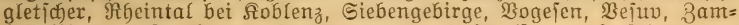

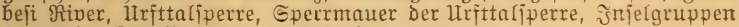
Santorin, \&aక ßalmas, 5̧elgoland.

B. Allgemeine geologifde Unterridtsfammlungen nad) Brof. A. Seim in 3üriấ):

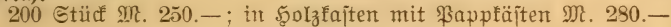
100 " 120.-;" " " 138.

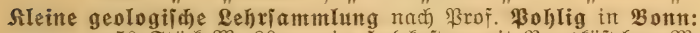

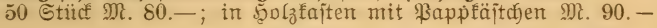

\section{Spezielle Geologie.}

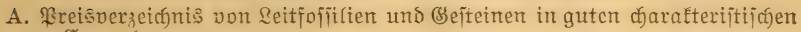
Exremplaren.

B. Sammlungen:

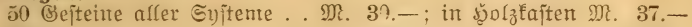

75

100

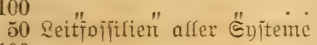

100

300

" " " 150. "

61.

78. -

20.-

36.-

$185 .-$

\section{Diapofitive für Geologie uno Paläontologie.}

હammlung für Den Interricht iu alfgemeiner S’eologie:

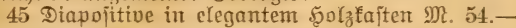
Eammlung für Sen Unterricht in hiftorijaber Scologie (Reitfojitien: Toula= Samntlung):

30 Dianofitive in elegantem Şolzfajtent M. 36.Einzelne Diapojitive. . . . . . je " 1.30

\section{Paläontologifcbe Gipsmodelle}

widftiger foijtien, forvie (3ipsabgüilie interefïnter Meteoriten und Bsold= flumpen, naturgetreut nad) den Sriginalen folortert. 


\section{Geologifdse Relief-profile aus Bips}

fonftruict von झrof. Dr. Duparc in Genf.

Bur Brundlage murben zwei parallele \$rofile eines (S)birges voer einer fictte

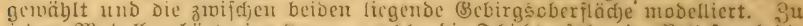

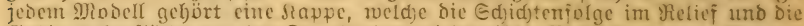
Sebirgsoberfläd)e vor ber Imudntion miedergibt. Freis̄ ber ganzen Eamm = lung von 8 Modellen פJ? 360.-.

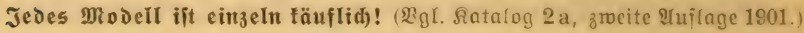

\section{Paläontologifoche Cebriammlungen.}

\section{AIIgemeine paläontologißðe Lebriammlungen.}

( aläozoologie und \$aläophytologie.)

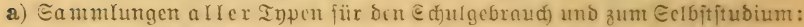
50 species $\mathfrak{M}$. 20.- 150 -pecies $\mathfrak{M}$. 75. $\quad 300$ species 9 ?. 150.$100 "$ " 50.- $200 "$ "100.- 400 " 225.b) Sammlungen innerbalb einzelner Iyper (Protozoa, Coelenterata, Echinodermata, Vermes-Molluscoidea, Mollusca, Arthropoda, Vertebrata).

c) Sammlungen nach Silafien (bzrw. Eubflaficn) (Spongiae, Anthozoa, Tetracora llia, Hexacorallia, Tabulata, Cnidaria. Crinoidea, Echinoidea ufw.)

(Bgl. Siatalog $2 b$, vierte 2 luflage 1901.)

II. Sammlungen zur Deizendenzlebre.

a) Eammlungen bes allgemeinen Metomorphismus.

b) Enmmlungen der altạmeinen progreffiven (Entrideflung

(2gi. Sintalog 2b, vierte थuflage 1901.)

\section{Mitroifopiłd) \$räparate.}

STleine હammlungen zu je 10 Dïnnidblifien von:

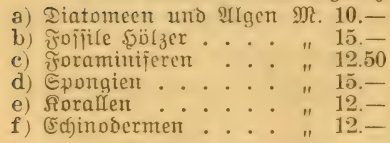

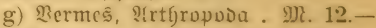
h) Brilozocil ..... "12.-

i) Brafiopusen ..." 12.

k) Mollusfen .... "12.

1) Bertebraten .... " " 12.-

Afrgemeine Gamulung von 110 Dïmidgliffen 20 . 130.-.

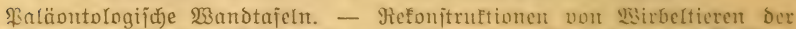

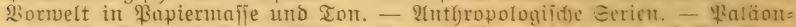
tologifge (Sipŝnodelle. - Ulle paläontolonij(hen vipuarate mo lltenjitien

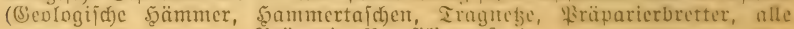
ßräparicr=Iltenfilien uim.).

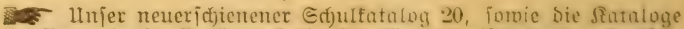

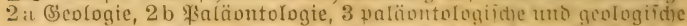

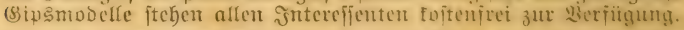
Einzelne gut erhaltene ₹ofitien poer ganze Eammlungen merben jeberzeit

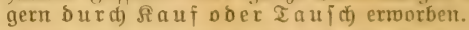

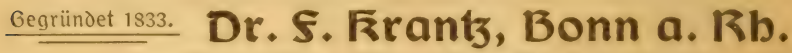
Rbeinifches Mineralien - Fontor.

Sabrik und Verlag mineralogifother und geologijotser Lebrmittel. 


\section{Sammlung Göschen}

6. Э. Görchen'fhe Verlagshandlung, Ireipzig.

\section{Derzeiđ̧̣nis der erjđ̧ienenen Bände.}

Seite

Aitronomie ....... 12

Bau= u. Ingenieurmifferffinaten 15 Biblithefsinejen . . . . 23

Botanif . . . . . . 10

厄hemte ........ 13

Ehemifhe Technologie . . . 14

Gleltrotedinit . . . . . 15

foritrirtichaft . . . . 21

Beologie ........ 11

Beographie ......6 6

(Beidichte ....... 4

Bemerberweien ...... 18

ఏandels̈nifienj(j)ait .... 21

\$ngiene ........ 23

Ingenieurtwifienjdjaiten . . 15

Surizprudenzz ..... 17

Saufmännifhe Wiffenfdjaftc: . 21

Sriftallographie . . . . . 11

Sunit ....... 20

Sanomirtictiaft ...... 21

Siteraturঠentmäler . . . . 3

Siteraturgef(dichte ..... 3

Mathematif ....... 8

Drectanit ....... 12

Dechanijळe Technologie . . 14
Meteorologie ..... 12

Militärmifíenjđjaft . . . . 22

Minteralogie . . . . . . 11

Mufifmiffeniđjaft . . . . 20

Ranurmifienjd) aft ... 9

Nautif . . . . . . . 17

Päbagogit ....... 19

Sharntazie . . . . . 23

Shilofophie . . . . . . 2

Whotograpjite ...... 23

Bhnfil . . . . . . . 12

Rechtsmilifenichaft . . . . 17

Religionzmifferichaft . . . . 19

Soztale Wiffenimaftelt ... 18

Spractroilfenidaft ..... 2

Staatsmilienidgaft . . . . 17

Stenographie ...... 23

Technologie, diemifine . . 14

Tedinologie, methanifdie . . 14

Theologie ....... 19

Bolfsmirtichaft $\ldots \ldots . .18$

Beichentunde .... 15 u. 20

8eitungsmejen ..... 23

8 oologie ...... 10 


\section{B. Verzeichnis nach Wiffenichaften.}

\section{Bibliothet zur Philojophie.}

Einfilfrung in bie Bhilofophic von Dr. Mar $\mathfrak{B}$ entider, Brofeffor an bet

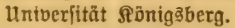

Rr. 281.

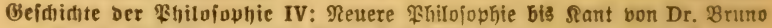
Baư), Rrivatboz. an ber Inniber. Salle a. S.

शr. 394. \$ind)ologie und \&ogit zur (Finfiifrung in bie \$hilofophie bon \$rofeffor Dr. Ih. Glienhants. Mit 13 Figuren.

Rr. 14.

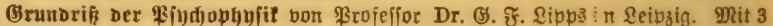
Figuren.

Gthit von $\mathfrak{B r o f}$. Dr. Thommas $\mathfrak{X}$ djelis in $\mathfrak{B r e e n .}$

Rr. 98.

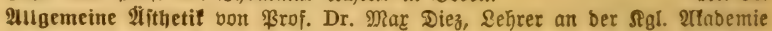
Der bildenden Rünfte in Stuttgart.

शr. 300.

Weitere Bände find in Vorbereitung.

\section{Bibliothef zur Epradwifienidaft.}

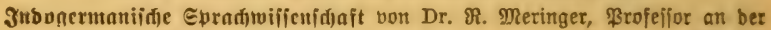
Univerjität હ̋raz. Mit 1 Tafel.

תi. 59.

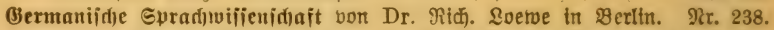
Romaniid) Evraf(uvifienid)aft von Dr. 2rolf Bauter, Privatbozent an ber Univeritiät $\mathfrak{B i e n .} 2$ Bände.

Rr. 128, 250. Eemitifac Euradtuificuidaft bon Dr. E. Brodelmant, Frofefior an ber

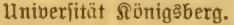

Mr. 291.

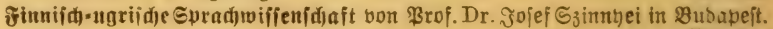
Deutiche (3rammatif und furze Beidichte ber beuticten Sprache bon Sir. 463. Brofeffor Dr. D. 'Inon in Dreasdelt. शr. 20.

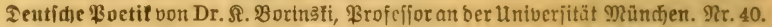

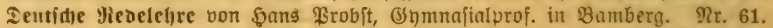

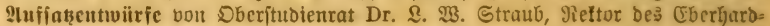

¿ubroigs=(5) muafiums in Stuttgart.

9r. 17.

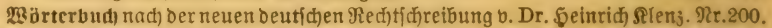

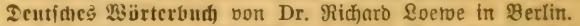

Rr. 64.

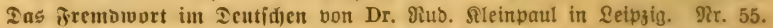

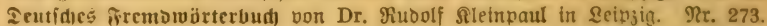

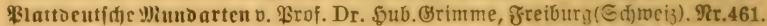
Ite beuticten leriouentumen von Dr. Rubolf Rleinpaul in Reipzig. Mr. 422.

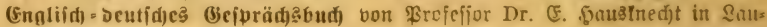
janne.

Mr. 424.

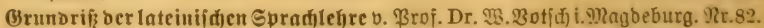
Ruffiige (Srammatit von Dr. Erid) Berneler, \$rof. an ber Uniberjit. Brag. Nr. 66.

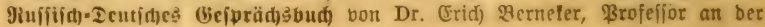
Щniverfität ¥rag. 


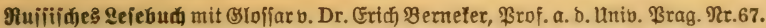

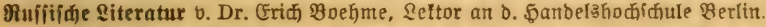

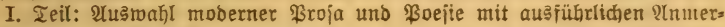
fungen und थifzentbezeidnung.

शr. 403.

- - II. Teil: Всеволодъ Гаршинъ, Разсказы. Mit 2 nmerfungen unઠ

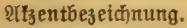

शr. 404.

Beidjidte ber tlafiifiden \$Gilologie bon Dr. Wiff. Rroll, oro. \$rof. an ber univerjität Muinjter.

9x. 367.

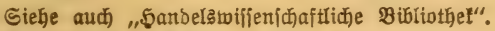

Weitere Bände find in Vorbereitung.

\section{Siteraturgeididtlide Biblinthef.}

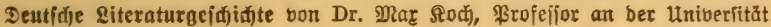
Breslaut.

शr. 31.

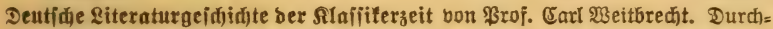
gejehen und ergänzt bon Sarl Berger.

SRx. 161.

Deutidje Qiteraturgeididjte be马 19. Jahrhunbert

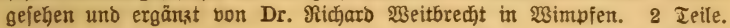

গ⿰. 134, 135.

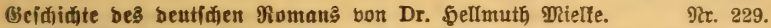

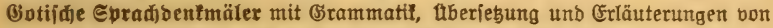

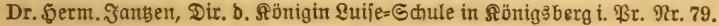

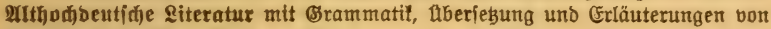
Th. Sđ)auffler, ßrof. am Realgnmnajum in Utm.

Nx. 28.

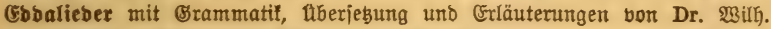

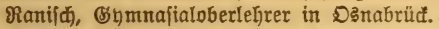

গr. 171.

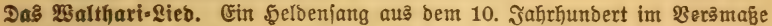

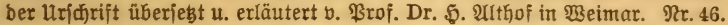

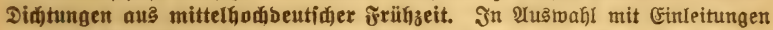
und Wörterbud) herauşgegeben von Dr. Sermann Jangen, Direftor ber

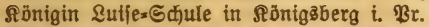

Nr. 137.

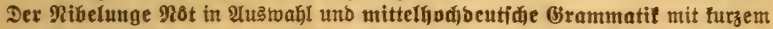

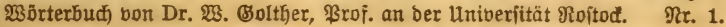

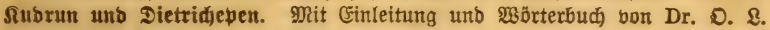
Siriczef, ßrof. an ber univerjität Müniter.

Nr. 10.

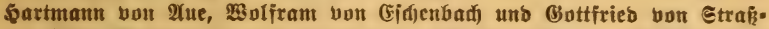

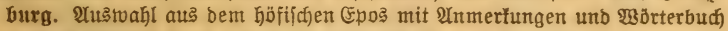

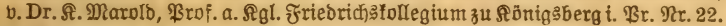

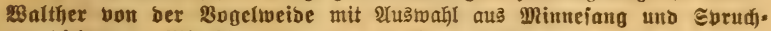

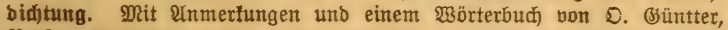

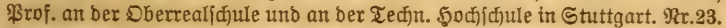

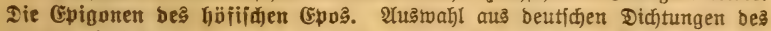

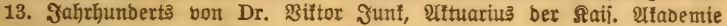
ber Wiffenfjaften in $\mathfrak{W}$ ien.

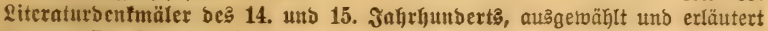

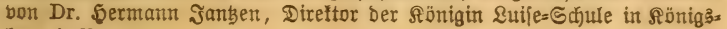
berg i. $\mathfrak{\$}$ r.

Nr. 181. 
Litcraturocnfmäler beß 16. Jalırhumberts. I: Martin \&uthex, ฐhomas

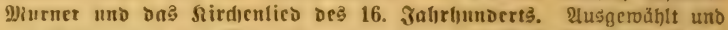
mit (Enlettungen und 2 nmerfungen verfeben von \$rof. (5. Derlit, Dber lehret am Nifolaignmnafium зи Seipzitg.

Nr. 7.

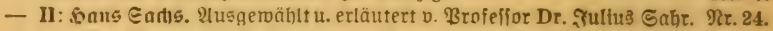

- III: Sion S3rant bis Jiollenhagen: Brant, \$utten, ifichart, fowie Tierepos

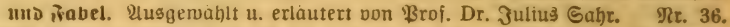

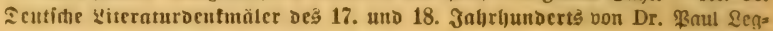
band in berlin. 1. Teil.

शr. 364.

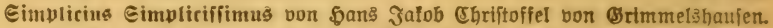
In थusmahl herausgegeben von ßrof. Dr. \&. Bobertag, Dogent an ber Univeritrat Rreslau.

शr. 138.

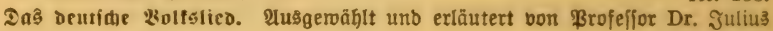
Sahr. 2 b̧änochen.

Nr. 25, 132.

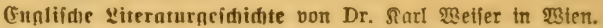

ఇr. 69.

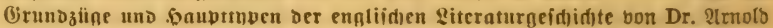

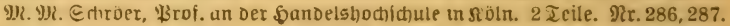

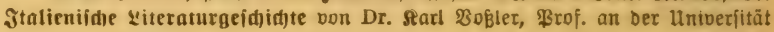
betbelberg.

शr. 125.

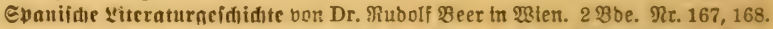
\{ormpiciifie Eitcranurncidjidte von Dr. Siarl von Vietnbaroftoetmer, Frof. an oer söntgl. Iechntichen Syochidhule Miund)en.

शr. 213.

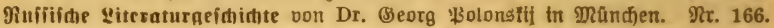

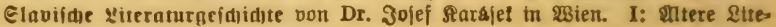
ranur bis zur Wsiedergeburt.

- II: Das 19. Tahrbunbert.

Mr. 277.

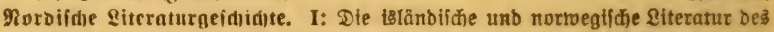

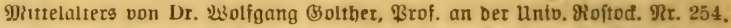

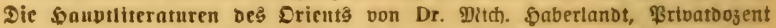
an Det Untverittat פsten. I: Die \&iteraturen Oftafiens uno Jnotens. शr. 162.

- II: Ire \&iteraturen Der Berier, Gemiten uno Türten.

Nr. 163.

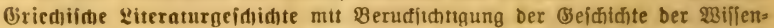
fchaften von Dr. 2lfted Bserfe, ßrof. an Der Untweri. Breifß̈mald. Mr. 70.

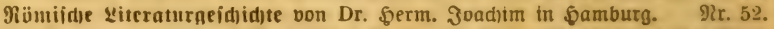

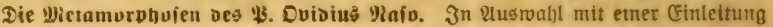
uno Unmertungen berausgegeben bon Dr. Jultus Biehen in frrantfurt a. $\Re$. Rr. 442.

Weitere Bände find in Vorbereitung.

\section{(Geidid)tlid)e Bibliotbef.}

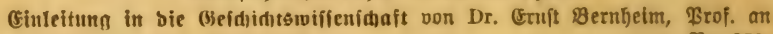
Der Untoerfitä! (siretformald.

भr. 270.

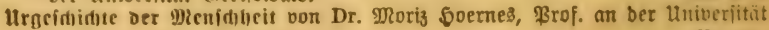
in waten. Dit 53 vibbiloungen.

शr. 42.

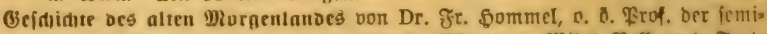
thichen Eprachen an ber Untwerfität in Mandjen. Mit 9 Boll= und Ierts bildern und 1 starte des Mtorgemlandes.

शr. 43.

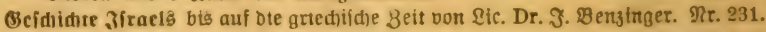




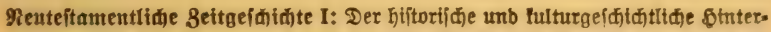
grunb bes Urdjriftentumts von \&ic. Dr. 2 . Staert, \$rofeifor an ber Uni= berfität Jena. Mit 3 \&arten.

शir. 325.

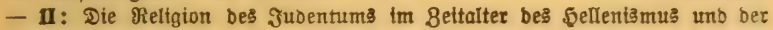

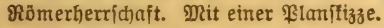

शr. 326.

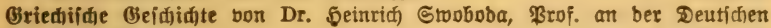
Unib. ßrag.

Mr. 49.

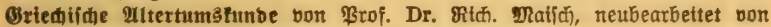
Reftor Dr. Franz Bohlhammer. Mit 9 Bollbilbern.

Nז. 16.

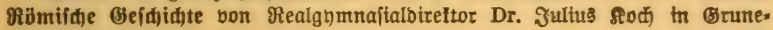
walb.

शז. 19.

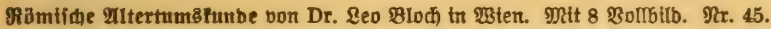

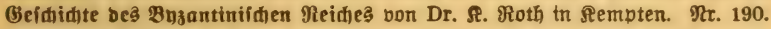

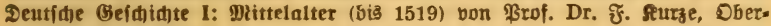
leb̆rer am $\Re g l$. Sutiengnmnaftum in Berlin.

Rx. 33.

- II: Seitalter ber Reformation uno ber Religiongిtriege (1500-1648)

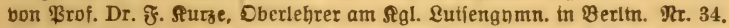

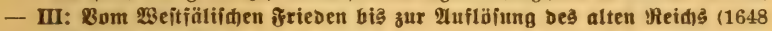

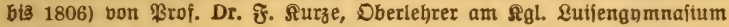
in Berlit.

Tix. 35 .

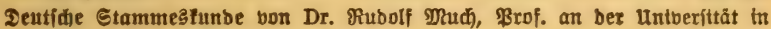
Bien. Mit 2 Sarten unb 2 Tafeln.

9ix. 126.

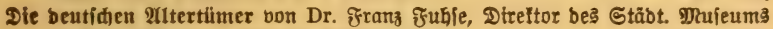
in Braunidimetg. MRit 70 2ubbildungen.

Ir. 124.

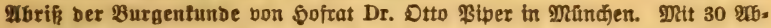
bilbungen.

Mc. 119.

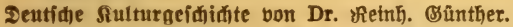

Nc. 56.

Deutíles Reben im 12. u. 13. Sahrhunbert. Realfommentar zu ben Bolfs unt Runitepen unb zum Minneiang. I: Dffentliches হeber. Bon Brof.

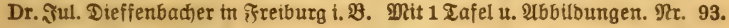

- II: ßrtbatleben. Mit 2rbbiloungen.

शx. 328.

Duellenfunde sur Deutiden B̉eididhte von Dr. Carl Jacob, ßrof. an ber Uniberjität in Tutbingen. 1. Band.

গ⿻. 279.

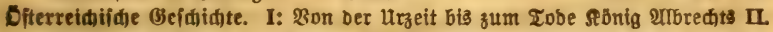
(1439) bon ßrof. Dr. Franz von Rrones, neubearbeitet bon Dr. Rarl ubltrz, ßrof. an ber Unib. Braz. MRit 11 Stammtafeln.

Nit. 104.

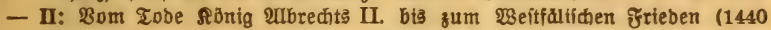
bis 1648) bon \$rof. Dr. Jranz oon Srones, neubearbettet von Dr. \&arl ublizz, Brof. an ber Uniberität Braz. Mit 2 Stammtafeln. Nr. 105.

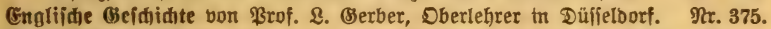

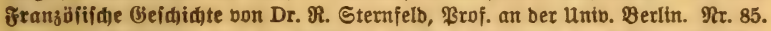

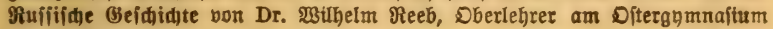
in Mains.

Bolnifide Bjefididte bon Dr. Elemens Branbenburger in ßojer. Rr. 4. Spaniidue Bieididite von Dr. Bsuift. Diertä. Nx. 338. Rx. 266.

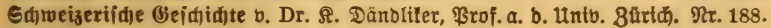
Geidid)te ber đrifitliden Baltanitaaten (Bulgarten, Serbien, Fumảnten,

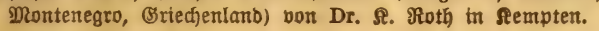

IR. 391. 


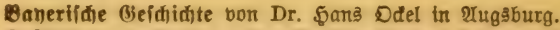

Nr. 160.

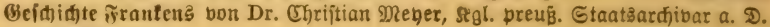
in Miunchen.

शr. 434.

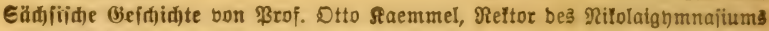
zи Leipzig.

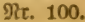

Thiltingifine (jeidjidjte bon Dr. Girnit Debrient in Jera.

Nr. 352.

Babifme (jcidjidjte von Dr. Sarl Brunner, ßrof. am (5)nnafium in ßrorzheim

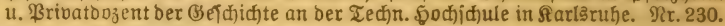

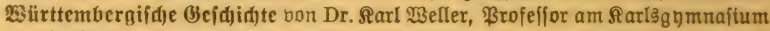
in Stuttgart.

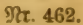

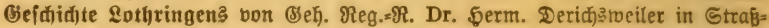
burg.

शr. 6.

Die ßultur ber Renaifiance. Befittung, Foriđjung, Diđjung von Dr. Robert F. 21rnolb, ßrofeffor an ber Uniberfität พBien.

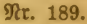

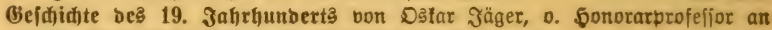
ber Univeritüt Bonn. 1. Bändđen: 1800-1852.

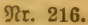

2. Bändchen: 1853 bis Enoe bes Jahrhunderţ.

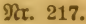

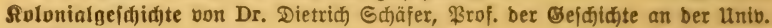
Berlin.

N(x. 156.

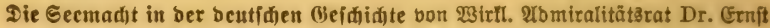
bon Salle, ßrof. an ber Uniberfitát Berlin.

Nr. 370.

0 Weitere Bände find in Vorbereitung.

\section{(Geographijde Biblinthet.}

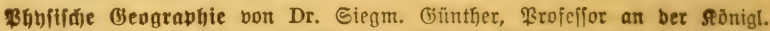

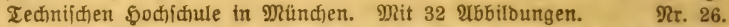
2(ítronomifije Breographie von Dr. Siegm. Günther, Brofeffor an ber Sënigl. Iechnticher bodichule in Mänchen. Mit 52 2rbbiloungen. 92. Slimafunde. I: Allgemeine Slimalehre von \$rofefior Dr. 23. Sïppen, Meteorologe ber Seemarte 5amburg. Mit 7 Tafeln u. 2 Fftguren. Nr. 114.

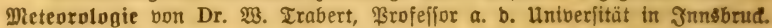
Mit 49 2rbbilbungen und 7 Iafeln.

Nr. 54.

Bhyfifde Meerestunbe von Brof. Dr. (Berharb Schott, 2rbteilungäborfteljer an ber

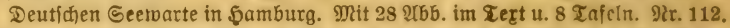

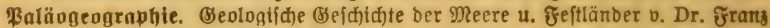
Solfimat in Wrten. Mit 6 Rarten.

Nr. 406.

Baläuflimatologie von Dr. Wilh. $\Re$. Edarbt in Aađjen.

Nr. 482.

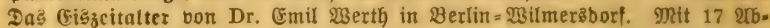
bilbungen unb 1 Sarte.

Nr. 431.

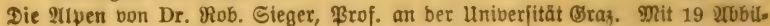
bungen unb 1 Sarte.

शr. 129.

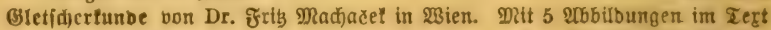
uno 11 Tafeln.

Rr. 154.

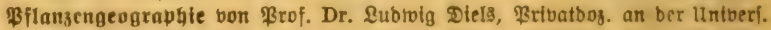
Berlin.

Mr. 389.

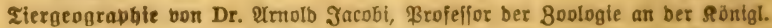
foritafabemie su Tharanbt. Mit 2 Rarten.

gir. 218. 


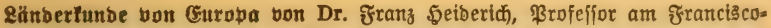
Sofephinum in Möbling. MRit 14 Texttärtdjen uno Diagrammen uno einer Sarte ber Prlpeneinteilung.

शx. 62.

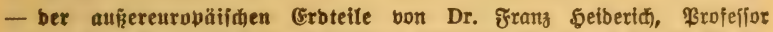

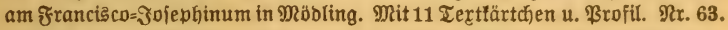

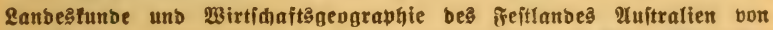

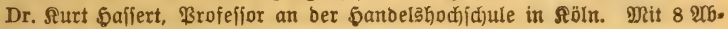
bildungen, 6 graphifien Iabellen und 1 Rarte.

शr. 319.

- von Baben von Brofeffor Dr. D. Sienits in Rarläruhe. Mit \$rofilen, SKbbildungen unb 1 Rarte.

Rr. 199.

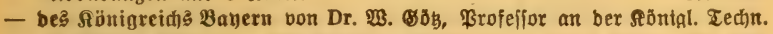

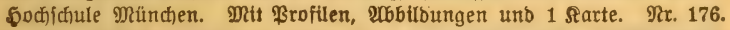

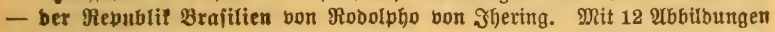
und einer Rarte.

Nx. 373.

- bon Britifd=: Rorbamerifa bon \$rofeffor Dr. 2 . Oppel in Bremen. Mit 13 2rbbilloungen und 1 Sarte.

Nx. 284.

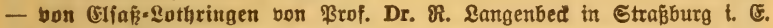
Mit 11 थbbiloungen unb 1 Sarte.

Nr. 215.

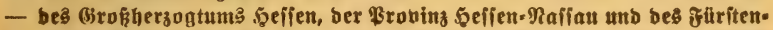

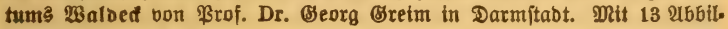
bungen unb 1 Rarte.

Nז. 376.

- ber Sberifitjen \$albinfel v. Dr. Frtb Regel, \$rof. a. b. Untb. Wurzburg.

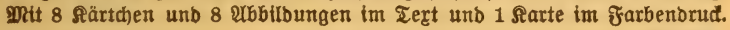

Nx. 235.

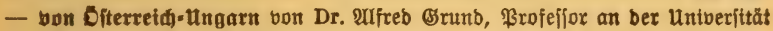
Berlin. Dit 10 Tertilluftrationen unb 1 Sarte.

Nx. 244.

- ber Rheinvroving von Dr. $\mathfrak{B}$. Stetnefe, Direftor bes Realghmnafum in Effien. Mit 9 Ubb., 3 Rärtçen uno 1 Rarte.

Nx. 308.

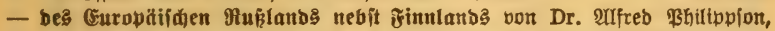
orb. \$rof. ber Ģeographie an ber Uniberfität Sualle a. S. Mit 9 2rbbildungen, 7 Texttarten unb einer litfyographifichen sarte.

शr. 359.

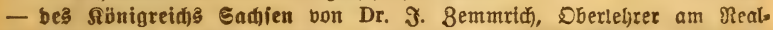

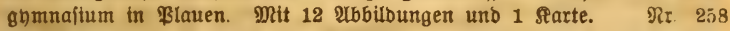

- ber Sanwei3 von \$rofefjor Dr. \$. Waljer in Bern. Mit 16 arbbiloungen unb einer Sarte.

शx. 398.

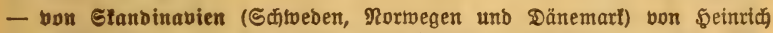
Serp, \&ebrer am (şmmafium uno \&ebrer ber crofunbe am Comentus. Seminar zu Bonn. Mit 11 थbbilbungen unb 1 Rarte. 202.

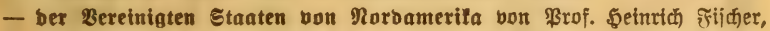

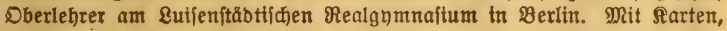
Jiguren im Text unb Tafeln. 2 gänbdien.

Nr. $381,382$.

- bes Rönigreids

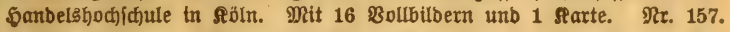
Die beutiden Solonien I: Togo und Samerun von \$rof. Dr. Sarl Dove. Mit 16 Iafeln uno einer litfographifich \$arte.

Nr. 441.

\&anbes. unb Boltstunbe \$aläitinas von \$ribatbozent Dr. B. Soljher in 5alle a. S. Mit 8 Bollbilbern unb einer Sarte.

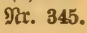




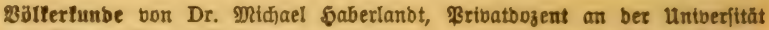
Wien. Mit 56 Qrbbillbungen.

शx. 73.

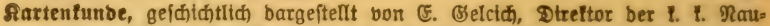
tifchen Schule in Suifinpiccolo, F. Sauter, Brofefior am Realgnmnafum in Ulm unb Dr. Baul Dinfe, 2rififtent ber (sejellidhaft far (5rDfunde in Berlin, neu bearbeitet bon Dr. M. Broll, Rartograph in Berlin. Mit 71 arbbilbungen.

Pr. 30.

Weitere Bände find in Vorbereitung.

\section{Mathematijae Bibliothet.}

Bjefdidte ber Mathematil bon Dr. $\mathfrak{A}$. Sturm, Brofeffor am Obergbmnaftum in Settenitetten.

Mic. 226.

Mrithmetil uno $\mathscr{A l}$ gebra von Dr. Бermann Sфubert, Brof. an ber Belebrten= ichule bes Johanneums in 5amburg.

Mr. 47.

Beifpiclfantmlung zur 2lrithmetil unb 2llgebra bon Dr. Dermann Schubert, \$rof. an ber (selefrtenid)ule bes Johanneums in samburg. Nix. 48.

AIgebraifde Surven bon Eugen Beutel, Eberteallebrer th Batbingen. (Eng. I: Sturbendialufíion. Mti 57 fitguren im Tergt.

$9 \pi .435$.

Determinanten von Baul 2 . Fifjer, Eberlebrer an ber Oberrealidule su (j) rog=staterfeloe.

शr. 402 .

C5bene Beometrie mit 110 ztweifarb. Figuren bon (5. Diabler, Brof. am (5) rafium in ulm.

פT. 41.

Daritellende Beometrie I mit 110 f̧iguren bon Dr. Rob. Баußner, ßrof. an ber Uniberitïät ふena.

- - I. Mit 40 Эiguren.

9x. 142.

Mr. 143.

Gbene un๖ iphärifide Iriponometrie mit 70 fłg. von Dr. Gerbarb Seffenberg, Brofefior an ber Lanbwirtichaftl. Ulfabemie Bonn. Bobbelß̧borf. $\Re$ r. 99.

Stereometrie mit 44 \&iguren von Dr. $\Re$. Blafer in Sturtgart. $\mathfrak{R}$. 97.

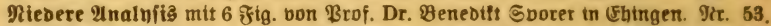
Bierftellige Iafeln unb Biegentafeln für lonaritbmifices und trigonometrifics Redinen in zmei farben fufammengeitelt von Dr. Siermann Sdubert, \$rof. an ber (selebrtenidjule bes Johanneums in Samburg. 9 r. 81 .

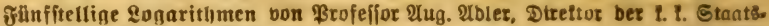
oberrealichule in $w_{3}$ ten.

शr. 423.

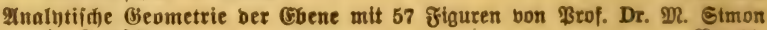
in Strabburg.

9x. 65.

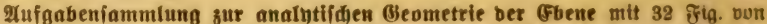

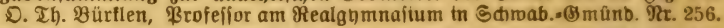
InaIntifide (Beometrie besి Raumesి mit 28 Ubbildumgen von ßrofeffor Dr. M. Simon in Straß̉burg.

Rr. 89.

2ufgabeniammlung zur analntijđiten Beometrie bes Raumes mit 8 Fig.

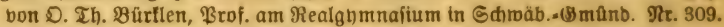

5objere Analyitis I: Differentialrednung mit 68 ftguren von Dr. Irieortd) Junfer, Brof. am Rarlsెgnmnafium in Stuttgart.

Rr. 87.

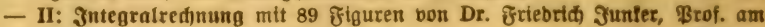
Sarisgnmnajium in Stuttgart.

Nr. 88.

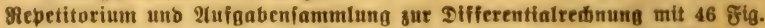

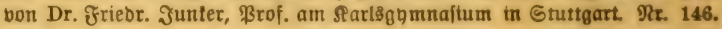




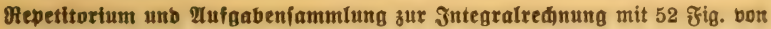
Dr. Frtebr. Sunter, Brof. am Sarlagymnafium in Stuttgart. Nr. 147. 9rojettive Bienmetrie in innthetificer Behandung mit 91 fig. bon Dr. R. Doehlemann, Brof. an ber Uniberittät MRüchen.

Rx. 72.

Mathematifine \$ิormeliammlung unt Fepetitorium bex Dathemati, enth.

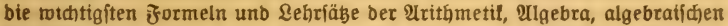
Qlnainfits, ebenen (seometrte, Stereometrie, ebenen uno fpharifithen Irtgono= metrie, math. Beographite, analnt. Bseometrie ber (5bene uno bes Raumes, ber Differential, und Integralrechung von D. Th. Bürflen, Brof. am

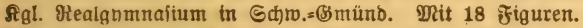

Nx. 51. Berfỉđerungsmathematil bon Dr. Alfreb \&oewy, ßrof. an ber univerjität Æreibur t. $8 \mathrm{r}$.

शx. 180.

Tusgleid)unģredung nad) ber Methobe ber Fleiniten Duabrate mit 15 Fftg. unt 2 Tafeln bon With. Weitbrecht, \$rofeifor ber Beodäfie in Stuttgart.

Rx. 302.

Seltoranalyfis von Dr. Siegfr. Baientiner, \$rtoatbozent für \$hyjił an ber Untoerfitåt Berltn. Dit 11 f̧iguren.

Pr. 354. Aftronomifide (Bsopraphie mit 52 §iguren bon Dr. Siegm. (3ünther, Brof. an ber Techn. bochichule in München.

Nr. 92.

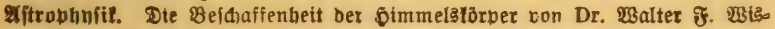

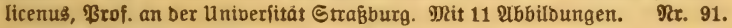
aftronomie. Bröвe, Bemegung uno Entfernung ber bimmelstörper von

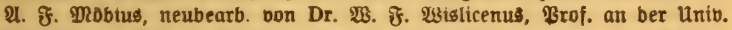
Straßburg. Mat 36 Ubbilbungen unb 1 Sternfarte.

Nx. 11.

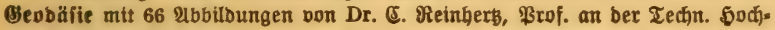
ichule bannover.

Nr. 102.

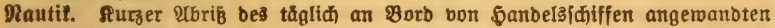
Ieils ber Gdiffahrtsfunbe mit 56 थbbudoungen bon Dr. Franz Schulze, Diteftor ber Naviaattonsichule su \&übed.

Nx. 84.

Geometrifides Beithen von \$. Becfer, 2ratiteft unb \&ehrer an ber Baus getwerfichule in Magbeburg, neu bearbeitet von ßrof. Э. Bonberlinn,

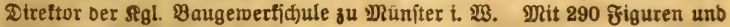
23 Iafeln im Text.

Nx. 58.

Weitere Bände find in Vorbereitung. Bleicbzeitig macht die Verlagshandlung auf die "Sammlung Scubert", eine Sammlung mathematifकer Cebrbücher, aufmerkiam. Ein vollifändiges Ver. zeichnis diefer Sammlung befindet fich am Scblufz diefes Droipektes. Aufzerdem kann ein ausfübrlicher matbematifoer katalog der 0. J. Göichen'iकen Verlagshandlung koitenfrei durd jede Buक. bandlung bezogen werden.

\section{Raturwifienidaftlide Bibliothet.}

Falăontologie und 2 bitammungslehre von ßrof. Dr. Sarl Diener in Fुier. DRit 9 Ubbilbungen.

शr. 460.

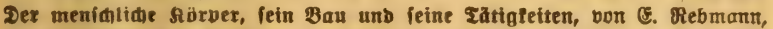
Dberíhulras in Sarlšruhe. Mit Befundhettslebre von Dr. med. \$. Setler. Dits 47 Ubbtibungen und 1 Eafel.

Nr. 18. 


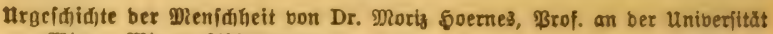
W3ten. Pit 53 abbiloungen.

No. 42.

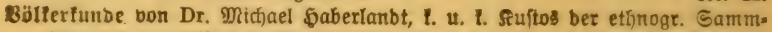
Iung beş naturhiftor. ఏofmufeums u. \$ribatbozent an ber Uniberịität ছisien. Mit 51 Ubbiloungen.

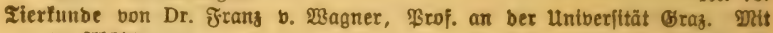
78 2ubbildungen.

शr. 60.

26rif ber Biologie ber Iiere von Dr. Seintid Simrotb, ßrofeffor an ber Uniberitiăt \&eipzig.

IT. 131.

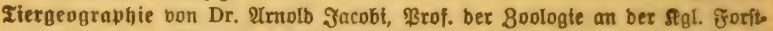
afabemie zu Tharanot. Matt 2 Sarten.

Nr. 218.

Da Tierreiđ). I: Säugetiere, bon Dberfubienrat ßrof. Dr. Surt \&ampert, Borfteher bes $\Re$ gl. Naturaltenlabtnetts in Stuttgart. Mit 15 2bbilb. Pr. 282.

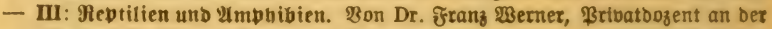
Untverfı̈ät Wien. Mat 48 abbilbungen.

Nx. 383.

- IV: Bitide, von Dr. Mar Rauther, ßrtbatbosent ber Boologte an ber Untverfität Gitȩ́en. Mit 37 Ubbilbungen.

গ⿰. 356.

- VI: Die wirbellofen Tiere bon Dr. Rubritg Böbmig, Brof. ber 8oologte

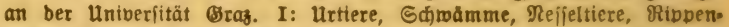

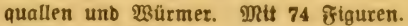

Nx. 439.

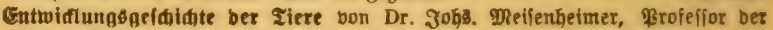

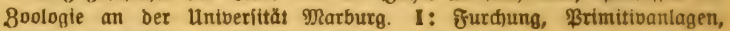

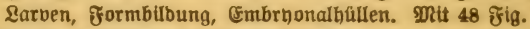

- II: Organbiloung. Mit 46 \&tguren.

शx. 378.

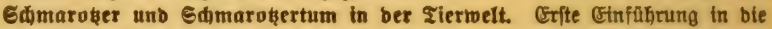
thertiche Schmaroberfunbe von Dr. Fram b. Wagner, ßrofeffor an ber

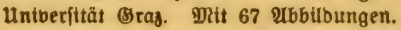

शr. 151.

Befajidte ber 3oologie von Dr. Rub. Burdharbt, wetl. Direltor ber Booldo. gitichen Station bes Beritner Rquariums in Robigno (Jitrient). Pr. 35 ?.

Die giflanze, thr Bau unb thr Leben von \$rofeffor Dr. (E. Dennert in OSobes. bers. פ⿴囗t 96 2rbbilbungen.

Nr. 44.

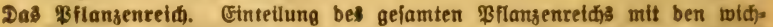

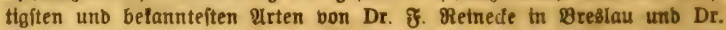
WB. Mitgula, Brof. an ber Frorftatabemie (Ftienach. DRts 50 fftg. Nr. 122.

Bflanzenbiologie von Dr. 23 . Pitgula, Brof. an ber froritalabemie (Ftienach. Dets 50 erbbilloungen.

शr. 127.

\$flanzengeograwhite von \$rof. Dr. Submig Diels, \$rtbatbog. an ber Univerf. Berlin.

Nr. 389.

Morphologie, 2Unatomie unb Bhbftologie ber Bflanzen bon Dr. 2B. Drigula,

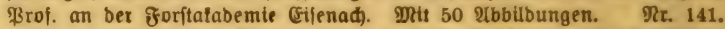

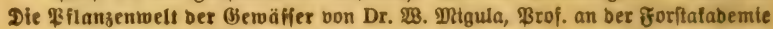
(Eilenad). Mat 50 2rbbildoungen.

গx. 158.

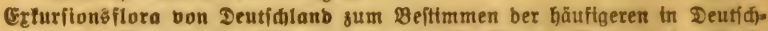
lanb rtibroactienten $\mathfrak{B}$ flarzen von Dr. 23 . Migula, $\mathfrak{B}$ rof. an ber forito alabemite (Etienac), 2 Tetle. פRt 100 Rbbildunger. $2 \pi .268,269$.

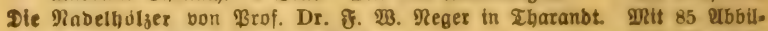
bungen, 5 Tabellen unb 3 Rarten.

शr. 355.

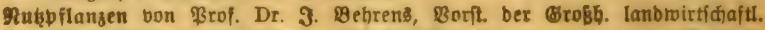
Berfud)కanit. 2luguitenberg. Mit 53 Figuren.

शr. 193. 


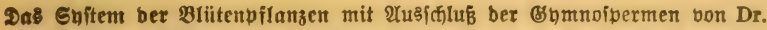

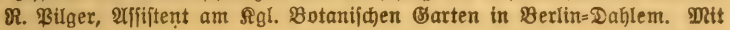
81 Figuren.

R̃. 393.

Bflangentrantheiten bon Dr. Werner Frtebrid Brud in Biteßen. Mit 1 farb. Tafel uno 45 2rbbilbungen.

Nx. 310.

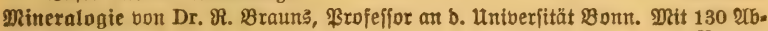
biloungen.

शr. 29.

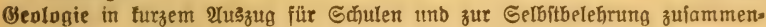
geftellt bon ßrof. Dr. (Eberh. Fraas in Stuttgart. Mit 16 2lbbilbungen unb 4 Iafeln mit 51 Figuren.

গ⿰. 13.

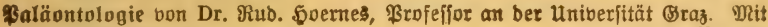
87 Ubbildungen.

शr. 95.

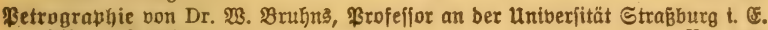
Mit 15 Ubbilbungen.

Nx. 173.

Ariitallographie von Dr. $\mathfrak{3}$. Bruhng, ßrof. an ber univerfität Straß̨burg. Mit 190 2lbbiloungen.

Nr. 210.

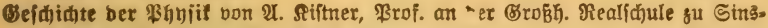

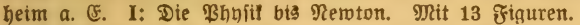

शx. 293.

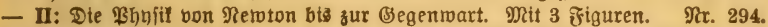

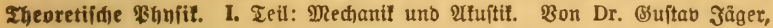

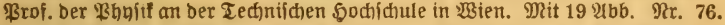

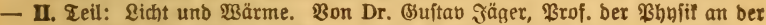

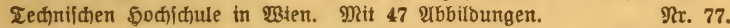

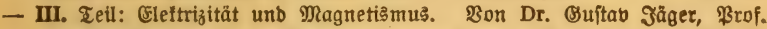

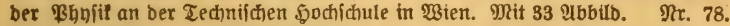

- IV. Ietl: (Eleftromagnetifhe \&idittheorie unb Eleftronit. Bon Dr. Buitav

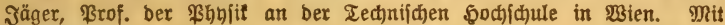
21 ริiguren.

Otabioaftivităt von 23 thh. Frommel. Mit 18 Figuren.

Mr. 374.

ghyiffalifde Mieifungsmethoben von Dr. WHifhelm Bahrst, Dberlehrer an ber Oberrealichule in (5roßs=\&ichterfelbe. Mit 49 Figuren.

গx. 301.

(5ejめidte ber Chemie von Dr. Sugo Bauer, 2rifitent am dem. Laboratorium ber Rgl. Iedniichen 5odid)ule Stuttgart. I: Bon ben älteiften Beiten bis zur Berbrentungstheorie von \&avoifier.

शr. 264.

- II: $\mathfrak{B o n}$ Ravotiter bis sur Bsegentwart.

Anoraanifice Chemie von Dr. Jof. Rlein in Mannheim.

Nr. 265.

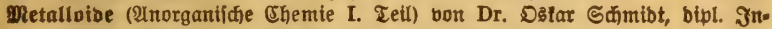
genieur, 2rififtent an ber $\mathfrak{A g l}$. Baugemerfichule in Stuttgart. $\mathfrak{R}$. 211.

Detalle (2norganija) (Chemie II. Ieil) von Dr. Ostar Sd)mibt, bipl. Inge:

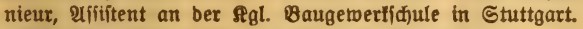

Drganiidje Chemie bon Dr. รof. Slein in Mannheim.

গ⿰x. 212.

Ohemie bex Soblenitoffberbinbungen bon Dr. Sugo Bauter, 2ijifítent am あem. Saboratorium ber Sigl. Iechn. 5ochidjule Stuttgart. I. II: Xlipha= tilche Berbinbungen. 2 Tetle.

Nx. 191, 192.

- III: Rarbocnflijaje Berbinoungen.

Nx. 193.

- IV: Бeterocnflitiáe Berbindungen.

Nx. 194.

Qualntifine Chemie bon Dr. Johannes Soppe. I: Theorte unb Bang ber Unalnje.

- II: Realtion ber Metallotbe unb Metalle.

शx. 247.

ఇx. 248. 
Mlaß̧analyie von Dr. Dtto $\Re$ hhm in Stuttgart. Mit 14 Ftg. Nr. 221.

Zednifd-(Shemiidie 2nalnfe bon Dr. (3. Runge, \$rof. an ber (Fibgen. \$olntedin. Gchule in 8ürtd. MRit 16 Ubbiloungen.

গx. 195.

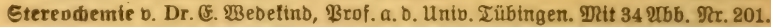
Allgemeine und phnfifalifite (Shemie von Dr. Mar Rubolphi, ßrofeffor an ber Iechn. Sodichule in Darmitabt. Mit 22 Figuren.

Pr. 71. Gleftrodiemie bon Dr. Seinrid Danneel in Frtedridshagen. I. Teil: Theoretifde

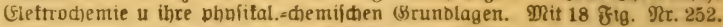

- II: Erperimentelle Elettrodjemte, Meß̉methoden, \&eitfäbrgteit, \&öfungen. Mit 26 Fipuren.

9?. 253.

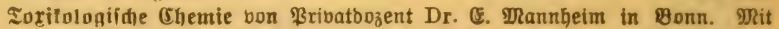
6 Ubbiloungen.

Rr. 465.

Inarifulturdicmie. I: Bflanzenernährung bon Dr. Sarl Brauer. Pr. 329.

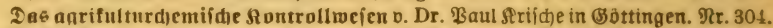
\$bufiologifde (Shemie oon Dr. med. $\mathfrak{A}$. Regahn in Berlin I: Rffimillation. Mit \& Tafeln.

Nc. 240.

- II: Diffitmitatton. Mit etner Tafel.

शr. 241.

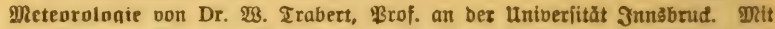
49 Ubbutoungen und ? Iafeln.

शr. 54 .

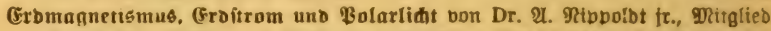

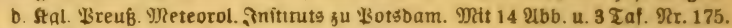
Aitronomic. ǴróBe, Betwegung und Entfernung ber Simmelsfỏrper von थ. Ff. Mäbtus, neu bearb von Dr. श3. ₹. Wristitcenus, ßrof. an ber Unib. Strapburg. Mit 36 Mihbildungen und 1 Stemfarte.

शx. 11. gitrophnfit. Die Beldaffenbeit oer 5immelstorper von Brof. Dr. ש3alter f.

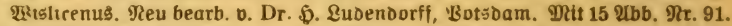
Aitronomifde Beographie von Dr. Stegm. (Bunther, Brof. an Der Iech)n. foctichule in ylundien. Mit 52 dbbiloungen.

शx. 92.

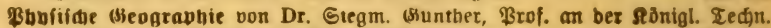
sodichule in viunchen. शRu 32 Ubbuoungen.

Rx. 26.

Bhyfithe Uleeregfunde von Brof. Dr. Berbarb Schott, Qbteilungsobriteher an ber Deutichen Seemarte in Samburg. Dait 28 Rbbutbungen tm Iert unb 8 Eafeln.

Nr. 112.

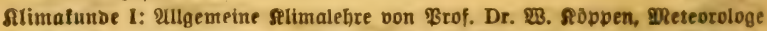
ber Seemarte Samburg. Dit 7 Iaf. u. 8 \&tg.

gr. 114.

- Weitere Bände find in Vorbereitung.

\section{Bibliothet zur Pbyjii.}

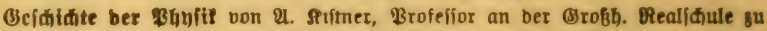

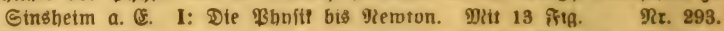

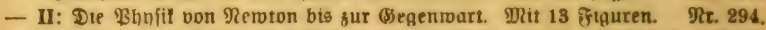

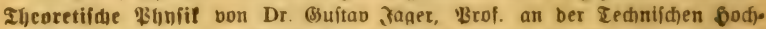

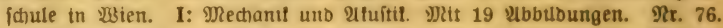
- II: Btat uno พ3ärme. Dit 47 2ubbtloungen.

- III: Eleftrtattát und Dagnetismus. Wtu 33 2tbbilbungen. Tr. 78. - IV: (Fleftromaanetif(t)e Stchttbeurte unb Eleftront? Wit 21 Figuren. Pr. 374. Rabioaftivitàt von פzilb frommel. Mut is ifiguren. givyfifalifde Miefiungsmethoden von Dr. WHabelm Habrbt, Oberlebrer an ber

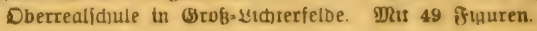
Rr. 301. 


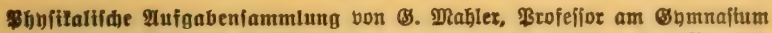
in $11 \mathrm{~m}$. Mit ben Refultaten.

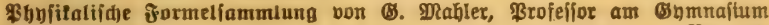
in $u l m$.

शז. 136.

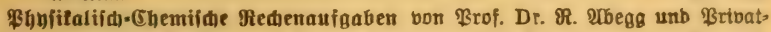
Dozent Dr. D. Sactur, betoe an Der Univerittắt breslaut. Nr. 445. Beltoranainfis von Dr. Stegit. Balentiner, \$rtbatbojent fitr \$hyfil an ber

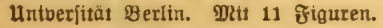

शx. 354.

Weitere Bände find in Vorbereitung.

\section{Bibliothef zur Chemie.}

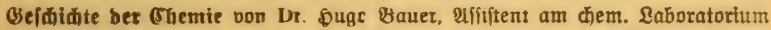
Der igl. Eechntichen fochichule Stuttgart. I: Bon ben älteften Beiten bis sur Rerbrennungstbeorte von \&avoifter. - II: gion Savoifter bis gur Begentwart.

2nornanifite (5hemie bon Dr. Jol. Sletn in Mannbeim. ఇr. 265. Metalloibe (2noraanifite (5bemie I) oon Dr. Ostar Sdimibt, bibl. Sngenteur, Uffiftent an bet Ral. Waugerwerfichule in Stuttgart.

शr. 211.

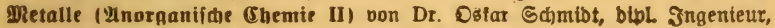
2lfifiten an ber stgl. Buugemerfichule in Stuttgart.

Drgantide Chemie oon Dr. SิDf. Siletn tn Mlantheim. St. 212. 9x. 38. Chemie ber Sohlenitofforbinoungen bon Dr. Sugo Bauer, 2rititent am chem. Saboratortum Der Sigl. Ied)n. Sochidule Stuttgart. I, II: Ulipha= tifice Berbindungen. 2 Teile. গx. 191, 192.

- III: Siarbocnflitiche Berbinbungen.

গi. 193.

- IV: ఏeterocnflitiche Berbindungen.

शr. 194.

Inalytifite (Shemie von Dr. Sob. Soove. I: Igeorie u. Sang b. Analyje. Pr. 247. - II: Reaftion bet Metallotbe unb Detalle.

NT. 248. Maß̧analnie von Dr. Dtto Röhm tn Gtuttaat. PRit 14 Fig.

গx. 221.

Ieduifh-(5hemiithe Inalpie von Dr. (3). Eunge, Brofeffor an ber (5togenöff. Bolntechn. Schule in 8 urtch. Mit 16 Ubbildungen.

gix. 195. Sterendemite von Dr. C. Bebetno, ßrofeffor an ber untverfitåt rübtngen. Dis 34 2ubtbunaen.

शx. 201.

2ulgemetne uno vunfifalifite Chemie bon Dr. Mar Rubolpht, ßrofeffor an Der Eechntichen bochichule tn Darmitabt. Mit 22 fitg.

Nr. 71.

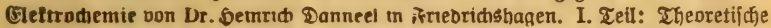
(Eleftrochemte u. thre phnitfaltith-chemtichen (3runblagen. Mit 18 ffig. Nr. 252.

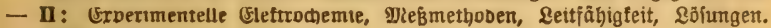
Mis 26 ริtquren.

शr. 253.

Zoritolopitime (Ehemie bon ßribatbozent Dr. C. Mannfeim in Bonn. Mit 6 Ubbubungen.

शr. 465.

2lgrtfulturchemie I: \$flanzenertåbrung von Dr. Sarl Đrauer. Nr. 329.

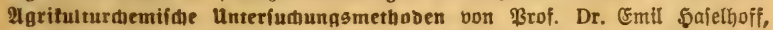

Borfteher Der lanomertichaftl Berfuchsftation in Marburg t. \$. शx. 470.

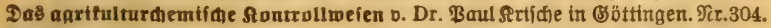

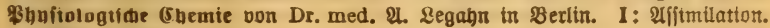
Yitu 2 Eafeln.

Nx. 240.

- II: Dtf́tmuaron. פit 1 Tafel.

शr. 241. 
Stödiometrifdie Irafgabeniammlung bon Dr. 2Bilhelm Bahrbt, Dberlehret

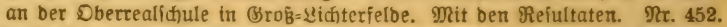

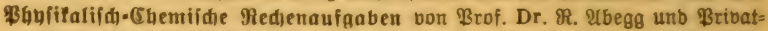
Dozent Dr. D. Sacfur, beibe an ber Uriberfität Breslau.

Rr. 445.

Da Siebe auch "Zechnologie". Weitere Bände find in Vorbereitung.

\section{Bibliothet zur Ied)nologie.}

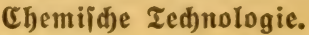

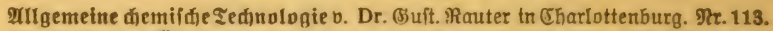
Die Eette uno Ele fowie bie Seifen. uno Serzenfabrifation uno bie Garge,

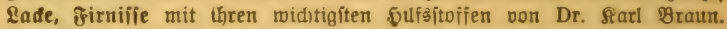

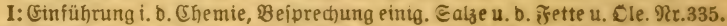

- II: Die Eeifenfabrifation, Die Seifenanalnfe und Dte Rerzenjabrifation. Dat 25 Ubbildungen.

Nx. 396.

- III: Бarze, Lađe, Fimtifie.

গx. 337.

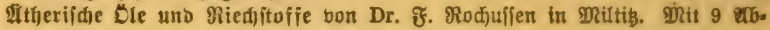
bilbungen.

शx. 446.

Die Cxblofivitoffe. Einflifrung th bie Chemie ber erbloftben Borgange vou Dr. \$. Brunstrig in Neubabelaberg. \$it 16 2rbbildungen.

शr. 393. Brauereiweien I: Mälzerei von Dr. Baul Dreberhoff, Direftor ber Brauet.

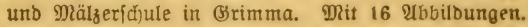

গ⿻r. 303.

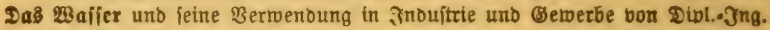
Dr. Crnft Reher. Mit 15 Ubbildunger.

PR. 861 .

Bafier unb 2tbwäifier. Thre Bufammenfebung, Beurteilung unb unteriuchung bon Brof. Dr. Emil Sajelhoff, Boriteber ber landwirtidjaftlidjen Ber. fucisitation in Marbura in fiefien.

Nז. 473.

2norganifide demiidse Snbutrie bon Dr. Buit. Rauter in Sharlottenburg.

I: Die Reblancjooainouitrte und thre Mebenzmeige. Mit 12 Iafein. Mr. 205.

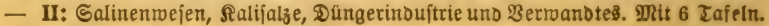

- III: Unorganifđje Ehemiiçe ßrăbarate. Mit 6 Tafeln.

शr. 206.

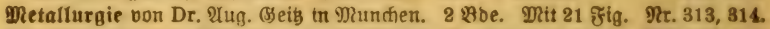

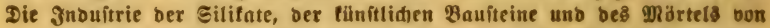
Dr. (Suitav Rauter. I: (5las= uno teramijde Jnouitrie. Dit 12 Iaf. Mr. 233.

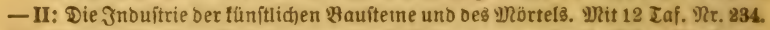
Die Zeerfarbitofie mit befonberer Berhdithtiaung ber fanthetifhen Dethoben

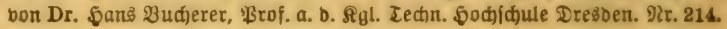

\section{Iteđanijळe Iednologie.}

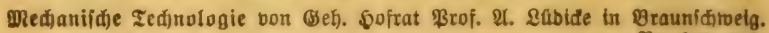
NR. $340,341$.

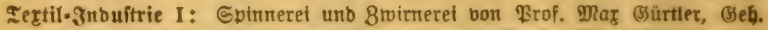

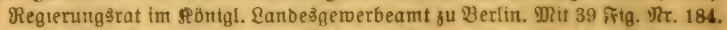

- II: Weberei, 23 irferet, $\mathfrak{B}$ :

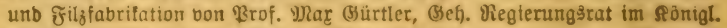
\&anbesgemerbeamt zu Berlin. פit 27 f̧iquren.

クr. 185. 


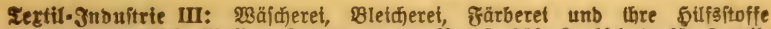

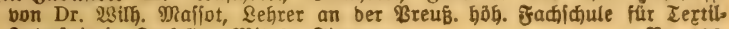

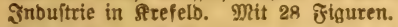

शr. 186.

Die Materialien bes Maid)inenbaues uno ber (Gletrotednit von Ingenteur

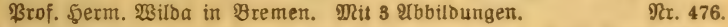

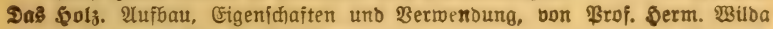
in Bremen. Mit 33 थbbilbungen.

Pr. 459.

C. Weitere Bände find in Vorbereitung.

\section{Bibliothef zu Den Jngenieurwilienid)aften.}

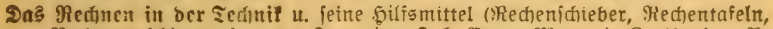

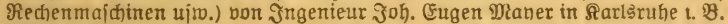
Mit 30 $26 b$.

Nr. 405.

Materialprïfungsweien. (Finführung in bie moberne Tedintl ber Matertalprüfung von $\Re$. Memmler, Diplom=Ingenteur, ftúnb. Mitarbetter am $R g l$. Material=

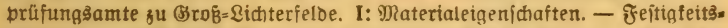

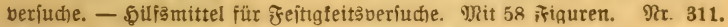

- II: Metallvräfung uno ßrüfung von Silfsmatertalien beg Maichinenbaues. - Baumaterialprüing. - ßapterprüfung. - Schmtermittelprüfung. Finiges fuber Metallographie. Dit 31 Fitquren.

Pr. 312. Mtetallographie. Rurze, gemeinfaßilthe Darftellump ber \&efre bon ben Die: tallen unt thren Legierungen, unter befonderer Bertafitchtigung ber Metallmifroffopie bon Brof. (F. Senn unb Brof. D. Bauet am $9 g l$.

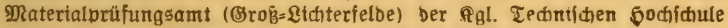
8u Berlin. I: Ullgemeiner Ieil. DRit 45 थbbuloungen tm Eerft unb 5 Sichtbildern auf 3 Eafeln.

शr. 432.

- II: Spezieller Teil. Mit 49 Xbbilbungen im Tert unb 37 \&idtbilbern auf 19 Tafeln.

Nr. 433 .

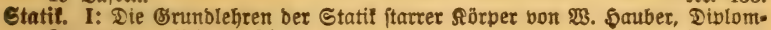
Ingenteur. Mit 82 ซึiguren.

II: Ungewanbte Statif. Mit 61 Fig:'ren.

Nr. 178.

శeftipteitaloure Nr. 179.

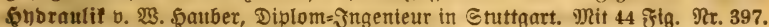
Benmetrifies Beidnen von \$. Becfer, 2lrchtteft unb Rehrer an ber Bau. getwerfichule in Magdeburg, neubearbeitet von \$rofeffor 3 . Bonderlinn in Münfter. Mit 290 \&iguren uno 23 Tafeln tm Tert.

Nr. 58.

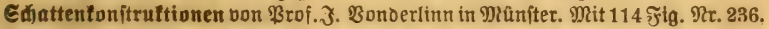
Barallelperipeftive. Rechtroinflige und ichtefminflige Aronometrie von Brof. §. Bonberlinn in פRürter. Mit 121 Figuren.

Nr. 260.

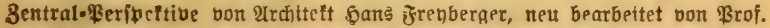

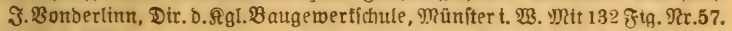

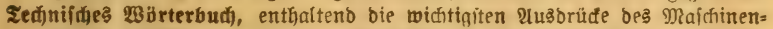
baues, Sdiffbaues und ber (Eleftrotec)nil bon (Erid) Srebs in Berlin.

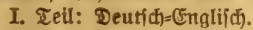

शx. 395. II. Teil: Englitich= Deutích.

Nx. 396.

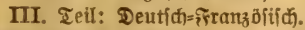

Nr. 453.

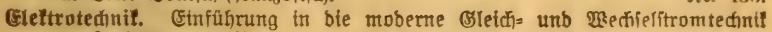

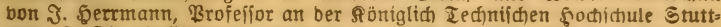

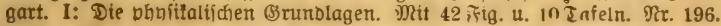

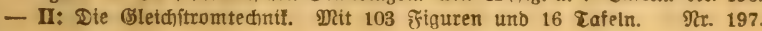


(Fleftrotechntt. III: Die Wechielftromtechntf. פit 126 fftg. u. 16 Iaf. Nr. 198.

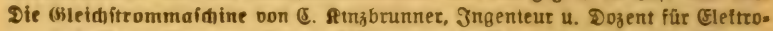
technif a. D. Jjumtrual School of Zechnologn in Mancheiter. MRit 7RFitg. Mrr.257. Eträme unb Spanmungen in Starfitromnetsen bon Diplom. Elettroingenteur

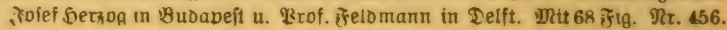

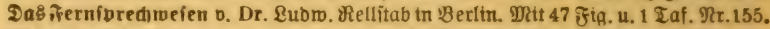

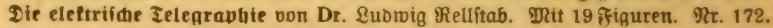

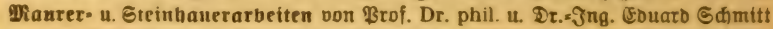

in Darmitabt. 3 Bänochen. Mit vielen 2lbbilbungen. Nr. 419-421.

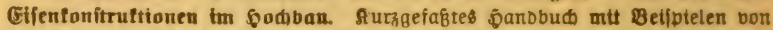
Ingenteur Parl Sđinder tn MetBen. Mit 115 Fjtguren. 322.

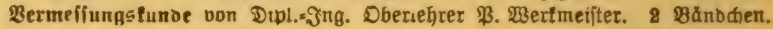
Phit 255 Ulbbilbungen.

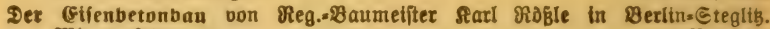
Dett 77 Qlbbubungen.

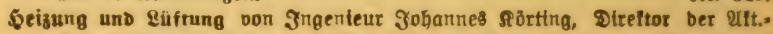

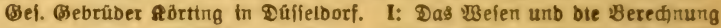
ber Setzungs: unb \&uffungsanlagen. WRit $\mathbf{3 4}$ Ftguren. 2 Rr. $\mathbf{3 4 2}$.

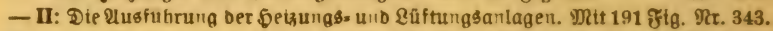

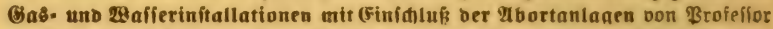

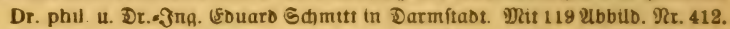

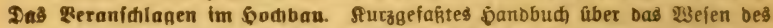

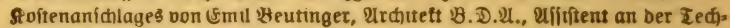
michen Sochichule in Darmitabt. Dit vielen Figuren.

Ne. 385.

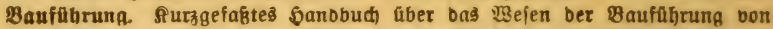

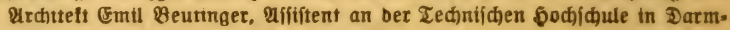
ftabt. Mat 25 iFtquren unb 11 Tabellen.

शx. 399.

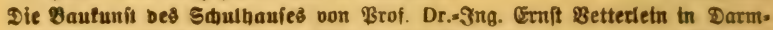
ftabt. I: Das Shulbaus. IDtt 38 थbbilbunger. 443. - II: Dte Schulråume. - Die Rebenanlagen. Wit 31 Vtöbtlbungen. Nr. 444. Dffentlime thabe und Samimmanitalten von Dr. Racl 23olff, Stabt=Oberbaurat in Sannover. Dett 50 žta

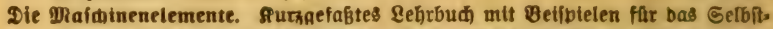
ftubtum unb Den praftition (Sebraud) von \&riebrid Barth, Dbertigenteur tn Nürnberg. Mats 86 g̊tguren.

शז. 3.

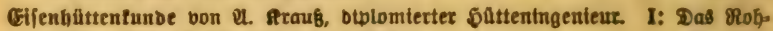
etjen. פits 17 Bftguren unb 4 Tafeln.

- II: Das Sdmmtebeifen. DRt 85 Rriguren unb 5 Tafeln. 9. 152. शr. 153.

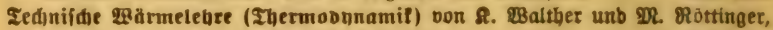
Diblom=ingenteuren. Dit 54 ङुtguren.

Nr. 242. Die Dambimaínine. Surzgefaßres \&ehrbud mit Betipteien fur bas Selbfiftubium u. D. praft. (Sebraud) v. Frtedr. Barth, Obering., Nurmberg. Mit 48 ङig. Pr. 8.

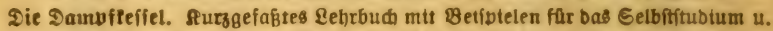
ben praft. (3ebraud) v. Erttebr. Barth, Obering., Nathrmberg. Witt 67 itg. Mr. 9. Die Bastraftmaitinen. Sturzgefabte Daritellung ber widittgiten (Sasmaichinen= Bauarten v. 3ngenteur Qlffred Sirichte in 5alle a. S. Datt 55 Ftguren. Pir.316.

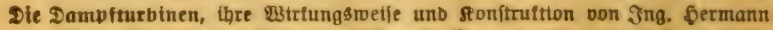
23ilda, Brofeffor am ftaatl. Ted)nifum in Bremen. MRt 104 थbb. Nr. 274. 


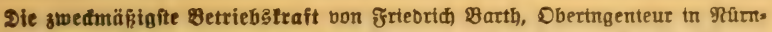
berg. I: (Einleitung. Dampftraftanlagen. Beridtedene Straftmaf́tmen. פRit 27 Qrbbilbungen.

Rc. 224.

- II: (5ass, שืafier unb

- III : Fleltromotoren. Betriebstoitentabellen. (5raphtiche Daritellungen. B̧aদ)! ber Betriebstraft. Mit 27 2lbbiłounaen.

Nr. 471.

Die Sebezenge, thre Sonftrultion unb Beredhung bon Sngenteur bermann Wstiba, ßrof. am ftaatl. Tednifum in Bremen. DRit 399 UdbbilDunger.

ग2. 414.

9umpen, Gubraulfidie unb pneumatifie 2 nlagen. Cin furzer aberbltd von Regierungşbaumeifter Rubolf $\mathfrak{B}$ ggbt, Oberlehrer an ber Rōntgl. höberen Maichinenbaufchule in $\mathfrak{B o i e r .}$ Dis 59 थbbtloungen.

פic. 290.

Die lanbwirtibaftlitien Mraidinen bon Sarl WBalther, Diblom=? Ingenteur in Mannbetm. 3 Bảnbdien. Mit vielen Ubbulbungen.

Pr. $407-409$.

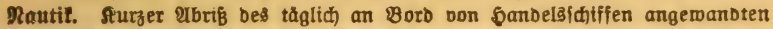
Zetis ber Sdiffahrtsfunbe. Bon Dr. Franz Sdulze, Dtrettor ber Ylavts

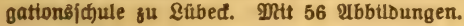

RT. 84.

\section{Eveitere Bānde find in Vorbereitung.}

\section{Bibliothef zu Den Redts= u. Etaatswifienidaften.}

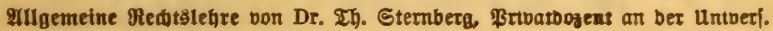
\&aufanne. I: Die Mletjobe.

शז. 169.

II: Das Sufitem.

9ix. 170.

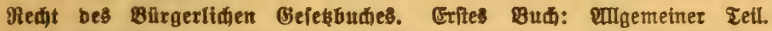
I: Finleitung - Rehre bon ben Berionen unb von ben Sahen von Dr. Baul Dertmann, Brofelfor an ber Untiberfitãt Erlangen. 147.

- II: Crmerb unb Berluft, Beltendmadiung unb Sdub ber Redite von Dr. Baul Dertmann, Brofeffor an Der Untverfttåt Eriangen Nr. 448. - 8twettes 2Bucf: Sđulbredit. I. 20bteilung: 2ulgemetne Rebren von Dr. Baul Dertmann, ßrofefior an ber untberíttät Erlangen.

शx. 323.

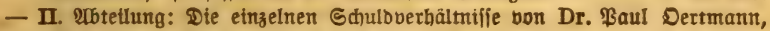
\$rofeffor an ber uniberfitåt criangen.

PR. 324.

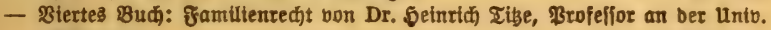
Bsotttingen.

Nit. 305.

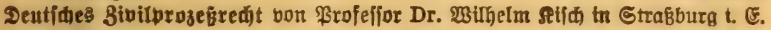
3 Bänbe.

Nr. $42 x-430$.

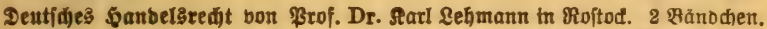
Nr. 457,458 .

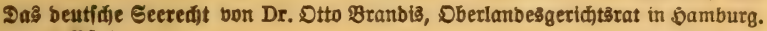

2 Bãnbe.

গtr. 386, 387.

Boitredit bon Dr. 2nfreb 2 olde, Boftnipeltor in Bonn.

Tr. 425.

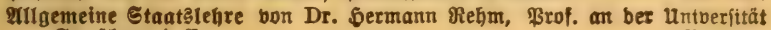
Straß̧burg t. E.

Pr. 358.

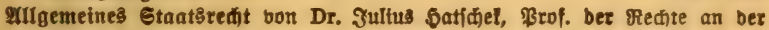
Rgl. Ulfabemie in $\Re$ ojen. 3 Bänbdien.

Nr. $415-417$.

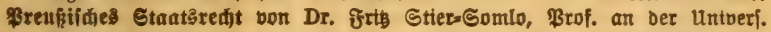
Bonn. 2 Telle.

Ntr. 298, 299.

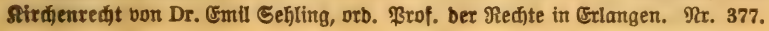




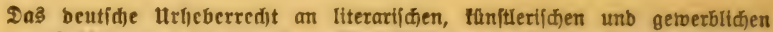

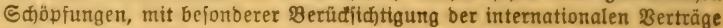
bon Dr. Buifav Rauter, ßatentantwalt in Eharlottenburg.

शr. 263.

Der internationale gewerblide Redtsidub bon $\Im$. Reuberg, Raiferl. Res gierungsrat, Mitglieb Des Raifert. ßatentamts zu Berlin.

গr. 271.

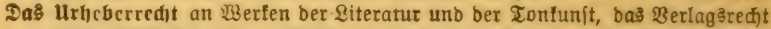

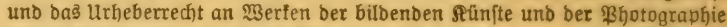

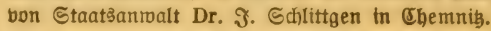

Nr. 361.

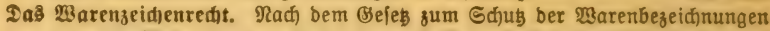
vom 12. Mai 1894 von Э. Reuberg, Raiferl. Regierunģ̧rat, Mitglieb bes Saiferl. ßatentamtes zน Berlin.

शr. 360.

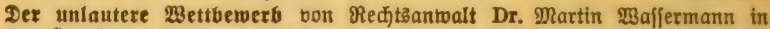
5amburg.

शr. 339.

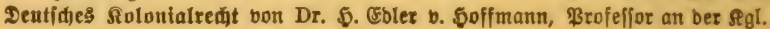
Ufabemte ßojen.

Ix. 318.

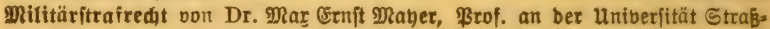
burg i. E. 2 Bänbe.

शr. $371,372$.

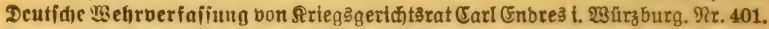

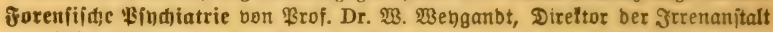
Friebridjberg in \$amburg. 2 Bänbchen.

গ⿰. 410 น. 411.

Weitere Bände find in Vorbereitung.

\section{Bulfswirtidaftlide Bibliothet.}

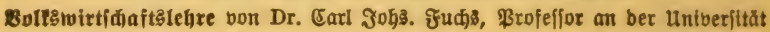
Ttubingen.

Nr. 133.

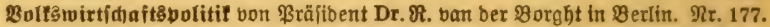

Gemerbeweien oon Dr. Werner Sombart, ßrofeffor an ber \anbel\$hod)idule Berlin. 2 Bảnbe.

शr. 203, 204.

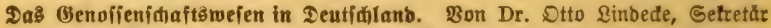
bes Sauptwerbanbes beuticher gerverblicher Benvifenidjaften. शr. 384.

Das Sandelsweien von Dr. Wilf). Rercts, ßrofeffor an ber Univerfität (Bôt: tungen. I: Das şandelsperional und ber W3arentjanbel. 296. - II. Die Cfffeltenbörje und bte innere Sandelsెpolitif.

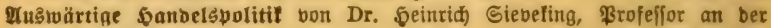
univerituât 8 ürtd).

शr. 245.

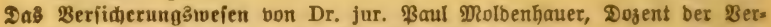
ficherungsmifieniahaft an ber 5anbelshodichule söln.

Rx. 262.

Die newerbline 21rbeiterfrage bon Dr. Werner Sombart, \$rofeffor an ber Sanbelshochichule Berlin.

Mr. 209.

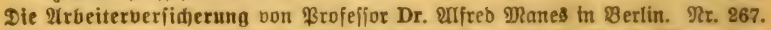

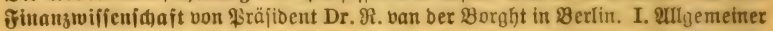
Teil.

Rr. 148.

- II. Befonberer Teil (Steuerlelire).

9x. 391.

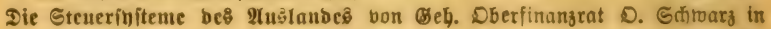
Berlin.

Nr. 426.

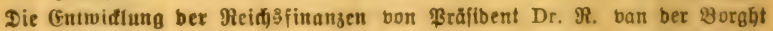
in Berlin.

शr. 427. 


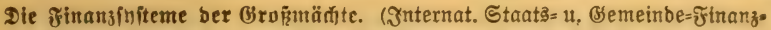
meien.) Bon D. Schtwarz, (Sch. Dberfinanzrat, Berlin. 2 Bbdi). Nr. 450, 451. Soziologie von Brof. Dr. Thomas 2 (đ)elis in Bremen.

Nr. 101.

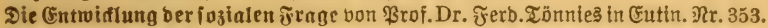
Irmenteien uno Armenfürforge. Einfüfrung in bie foziale ફilfsarbeit von

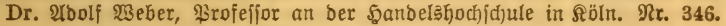
Weitere Bände find in Vorbereitung.

\section{Theologilde und religionswifienidaftlide Bibliothef.}

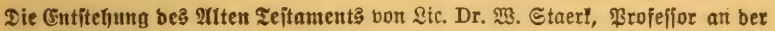
Uniberfität in ฐ̃ena.

शr. 272.

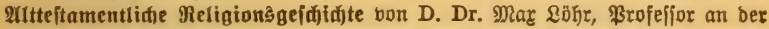
Univerítät Breslau.

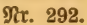

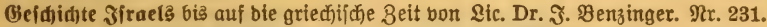

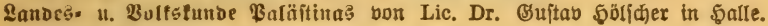
Mit \& Bollbilbern und 1 Rarte.

Nx. 345.

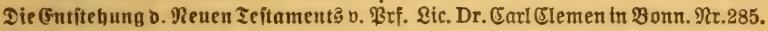

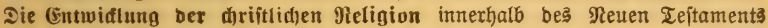
bon ßrof. Qic. Dr. Sarl Slemen in Bonn.

Nx. 388.

Reuteftamentlide Zeitgeidid)te von ßic. Dr. $\mathfrak{3}$. Staert, \$rofeffor an ber

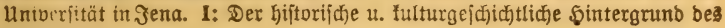
uxchriftentumb.

) 32 . 325.

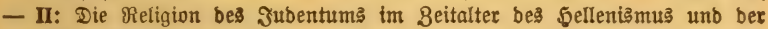
Römerherridiaft.

शr. 326.

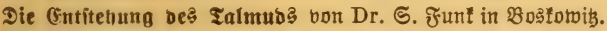

ఇr. 479.

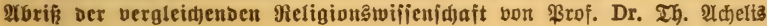
in Bremen.

Mr. 208.

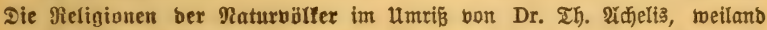
ßrofeffor in Bremen.

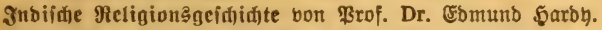

Buobha von ßrofefior Dr. Comund 5arby.

शx. 449.

शr. 83.

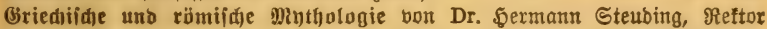

bes Jnmnafums in Sdineeberg.

शr. 27.

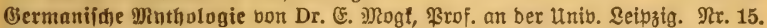
Die beutidje \$eldenfage bon Dr. Dtto Ruitpolo Siriczel, \$rofefior an ber Univerjität Münjiter.

Nx. 32.

Weitere Bände find in Vorbereitung.

\section{Pädagogijde Bibliothet.}

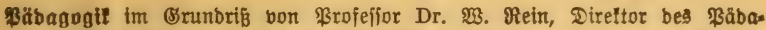

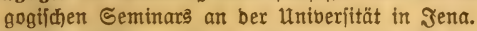

Rx. 12.

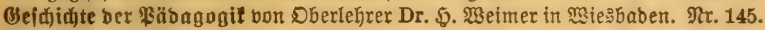

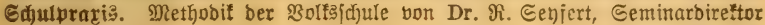
in Bidjopau.

Rr. 50.

Beidjenidule von ßrofeffor $\Re$. Stimmid in ulm. Mit 18 Tafeln in Tons, Jarbens u. Cுoldbrud u. 200 Bolls น. Tertbilbern.

शx. 39. 


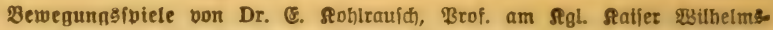
(5)mnafium zu 5annober. DRit 14 2bbtloungen.

Rx. 96.

Das affentlime Unterrimtsmeien Ieutidlanos in bet Biegentoart bon Dr. Baul Stonner, (Bomnaitaloberlehrer in Brotfau.

Nix. 130.

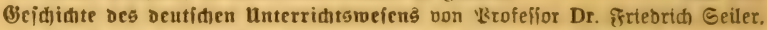

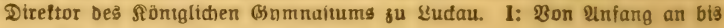
sum Ende Des 18. ? Tabrhunderts.

9x. 275.

- II: Rrom Reainn bes 19. Rahthunberts bis auf bte Meapminart. Nr. 276.

Das beutidie îortbilounasfitultweien nach fetner geichthtlichen Entrotdlung unb in feiner gegentaartigen (B)eftalt von 5 . Sierds, Direftor ber itabt. Frortbilnunasichulen tn şeide $i$. Solitein.

Ne 392.

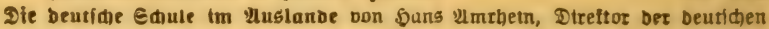
Sdule tn ruttic).

IT. 259.

Weitere Bände find in Vorbereitung.

\section{Bibliothef zur Sunit.}

Stillunbe bon Brof. \$arl Otto Sartmann in Sturtgart. פett 7 Bollbtibern und 195 Eertilluitrationen.

शr. 80.

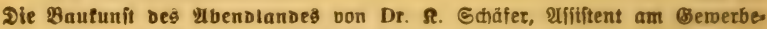
muleum tn Bremen. Wat 22 थbhubungen.

Rr. 74.

Die \$laitif bę Abendandes von Dr. Suns Sิtegmann, Dtreftor be\$ Banr. Ratonalmuieums in yiunchen Mrtt 23 इafeln.

Nr. 116.

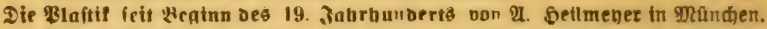
Dits 41 शुollbubern auf ameritunsichem stuifiorudpabter.

Nr. 321.

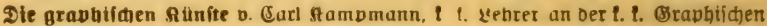

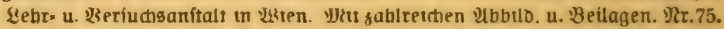

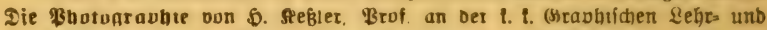

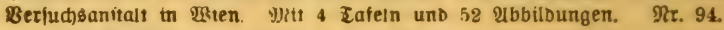

Weitere Bände find in Vorbereitung.

\section{Bibliothef sur Miufif.}

2rlaemeine Mufiflehre von Stephan firehl th Letpztg.

शx. 220.

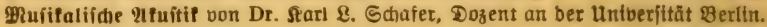
Det 35 थbbtloungen.

গ⿻. 21.

Sarmonielehte von $\mathscr{U}$. Salm. Mit vtelen Rotenbeilagen.

Nr. 120. Dhufifalifice isormentehre (.Rombofitionslehre) von Stephan Steb̆. I. II. Mit vielen Motenbetiptelen.

Nr. $149,150$.

Sontrabuntt. Dite Rebre von ber felbitäntgen Stmmfabrung bon Stephan Strebl in Retbztg.

शr. 390.

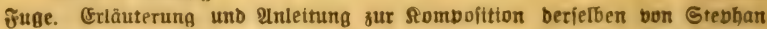
Strebl th Spipitg.

97. 418.

Inftrumentenlehre von Mufifotreftor Franz Manethoff in 5hemntb. I: Tert. II: Notenbetiptele.

Mr. $437,438$.

Mnufifäfthetif von Dr. S. Brunsth in Stuttgart.

शr. 344 .

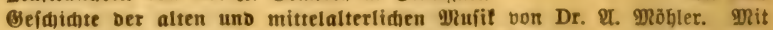
zablretchen edbbiloungen uno Muitfbetlagen. I. II.

शr. $121,347$. 


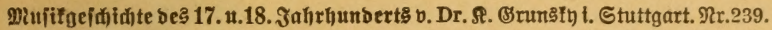

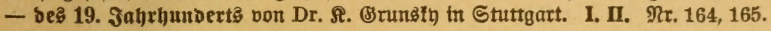

\section{Weitere Bände find in Vorbereitung.}

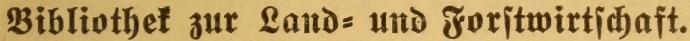

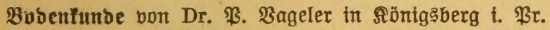

Nr. 455.

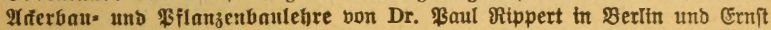

\&angenbed in Bochum.

Nr. 232.

\&anbwirtid)aftlidhe Betriebslehre bon Emit \&angenbed in Bochum. Nr. 227.

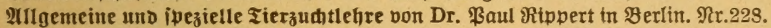
Agrifulturdiemie I: Bflanzenernahrung von Dr. Sarl Brauer.

Nx. 329.

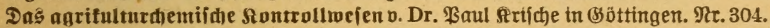

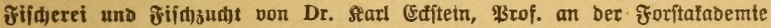
CEbersmalde, 2ubteilungsotrigent bet ber \$auptftation bes forfltthen Ber= juch)meiens.

Nr. 159.

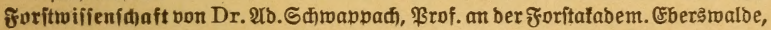

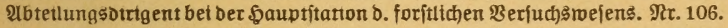

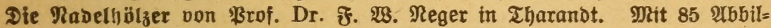
bungen, 5 Tabellen unb 3 sarten.

Nr. 355.

\section{Weitere Bände find in Vorbereitung.}

\section{Sandelswiffenid)aftlide Bibliothef.}

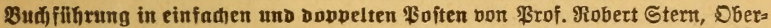
lebrer ber Dffentlithen Sandelslebranitalt unb Dozent ber Sandelshod)= id)ule zu Letpzig. Mat formularen.

Nr. 115.

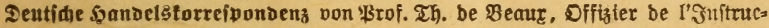
tion $\mathfrak{B}$ bubltaue, Dberlebrer $a$. D. an ber Dffentliden \$andelslehranitalt unb Settor an ber நandelshochichule zu Letpzig.

शr. 182. ชranzafifithe bandelstorrefpondenz von \$rofeffor $\mathfrak{T h}$. be Beaux, Offizter be l'snitruction 'Bublique, Oberlehrer a. D. an ber Offentltaten \$anbels= lehranftalt uno Rettor an ber ఏanoelshochichule zu Reipzig. $\mathfrak{R r} .183$.

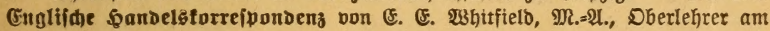

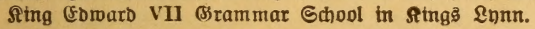

शr. 237. Stalienifide \$anbelatorreipondenz von \$rofefior थlberto be Beant, Dber=

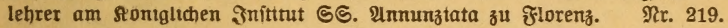
Spanifite \$andelstorreipondenz v. Dr. Alfredo Nabal be Mariezcurrena. Nr. 295.

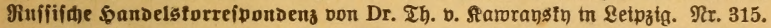

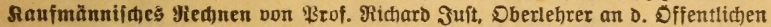

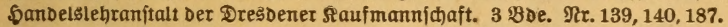

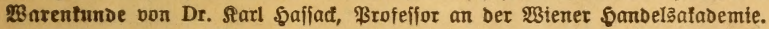

1: Unorganthe $\mathfrak{B a r e n . ~ M i t ~} 40$ थbbulounger.

ఇr. 222.

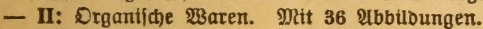

গx. 223.

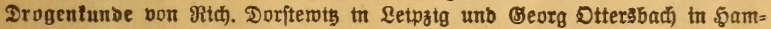
burg.

Nr. 413. 


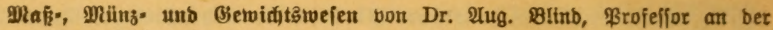
5anbelşđule in Söln.

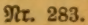

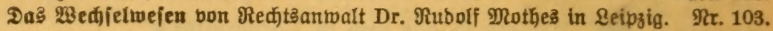

Deitere Bände find in Vorbereitung. Siebe auch „Volks= wirtichaftlide Bibliotbek". Ein ausfübrliches Verzeichnis der a úferdem im Verlage der $\mathbf{B}$. J. Göfकen'\{chen Verlagshandlung erfकienenen handelswiffenfकaftlichen Werke kann Jurch jede Bucbbandlung koftenfrei bezogen werden.

\section{Militär $=$ und marinewifieniðaftliđe Bibliothef.}

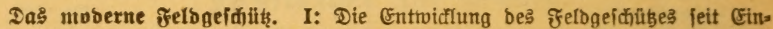

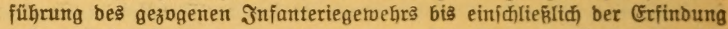

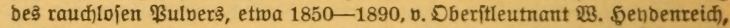

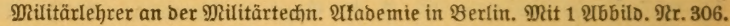

- II: Die Entroiflung bes heutigen Jelbgeiđübes auf Brunb ber Frfinbung

- bes raud)lojen $\mathfrak{B}$ ulvers, etwa 1890 bis zur (S)egentwart, bon Dberjtleutnant

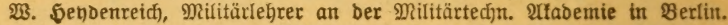
Mit 11 2bbildungen.

ఇr. 307.

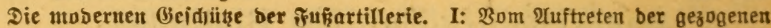

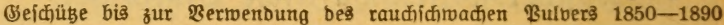

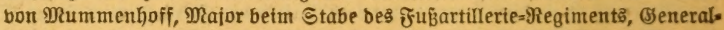
ferbzeugmeifter (Branbenburgitiches $\mathfrak{N}$ r. 3). Mit 50 - Tertbilbern. $\mathfrak{R r} 334$.

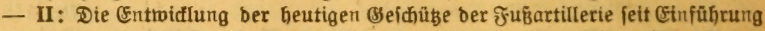

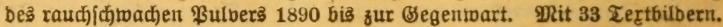
Rr. 362.

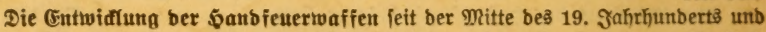

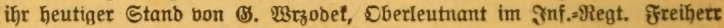

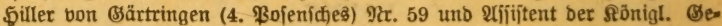
weyrbrüfungstommiffiton. Mit 21 2rbbiloungen.

Nr. 366.

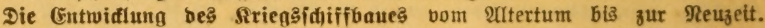
I. Teil: Dasె Beitalter ber Ruberidhiffe und ber Segelifoiffe für bie

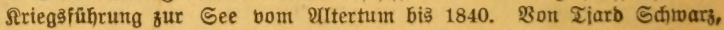
(Beh). Marintebaurat u. Schiffbau=Direftor. Mit 32 थbbildungen. $N$ r. 471. MRiritärîtrafred)t von Dr. Daç Ernit Maber, \$rof. an ber Uniberituät Straß̧= burg i. E. 2 Bänbe.

शr. $371,372$.

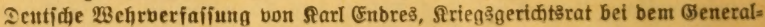
fommando bes $\Re g l$. banr. II. Urmeeforbs in \$3ürźburg. Rr. 401.

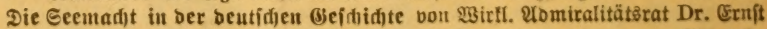
bon ఏalle, \$rof. an ber Univerjität מerlin. शr. 370. 


\section{Bibliothefs= und 3eitungswejen.}

Bolfabigliothefen (Büd)er= uno \&efehallen), ihre (Fintid)tung uno Berivaltung

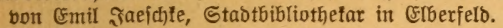

Nir. 332.

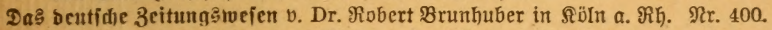

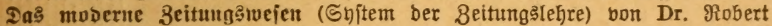
Brunbuber in $\Re$ öln $\mathfrak{a}$. $\Re$.

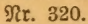

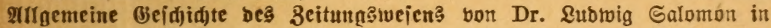
ฐena.

शr. 351.

\section{Sygiene, Mtedizin und Pharmazie.}

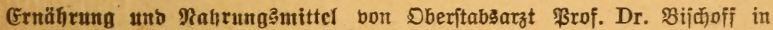
Berlin. Mit 4 Figuren.

Rr. 464.

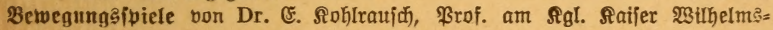

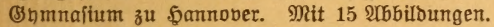

Rr. 96.

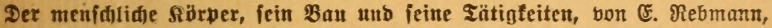
Dberichulrat in Sarlşruhe. Mitt (Sejundbeitslebre von Dr. med. \$. Seiler. Mit 47 2ubbildungen und 1 Tafel.

Rr. 18.

Die Infeftionstranfheiten uto ihre Berfjütung von Stabsarzł Dr. $\mathfrak{3}$. \$off= mann in Berlin. Mit 12 vom Berfaffer gezeidyneten 2lbbildungen uno einer fiebertafel.

शx. 327.

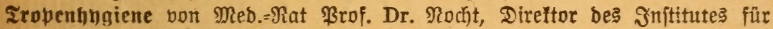
Schiffs = $u$. Tropenfrantheiten in Samburg.

Nr. 369.

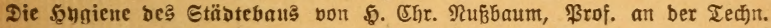
5odjidule in 5annover. Mit 30 2rbbiloungen.

Nr. 348.

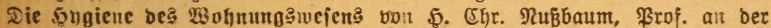
Techit. \$ochid)ule in \$ुannover. Mit 20 26billoungen. Oiverbehngiene von (Seh. Mebizinalrat Dr. Roth in \$otgbam. Nr. 350.

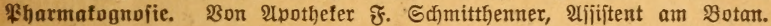

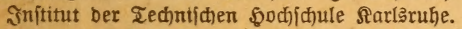

Nr. 251.

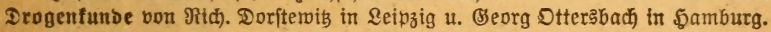

\section{Bhotographie.}

शr. 413.

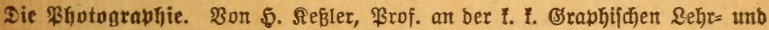

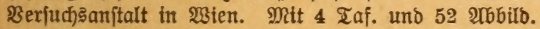

গr. 94.

\section{Stenographie.}

Stenographie nach bem Snftem bon f. $¥$. Babelgberger von Dr. 2nbert

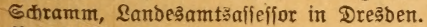

Nr. 246.

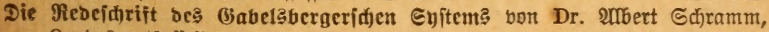
Landesamtsalfefior in Dresben.

গx. 368.

Lefrbud Dex Bereinfad)ten Deutid)en Stenograbhie (Finig.=Syitem Stolze=

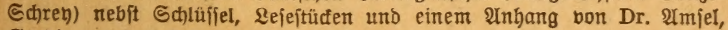
Stubientat bes Sabettenforps in Bentberg.

গt. 86.

Weitere Bände diejer einzelnen Abteilungen find in Vorbereitung. 


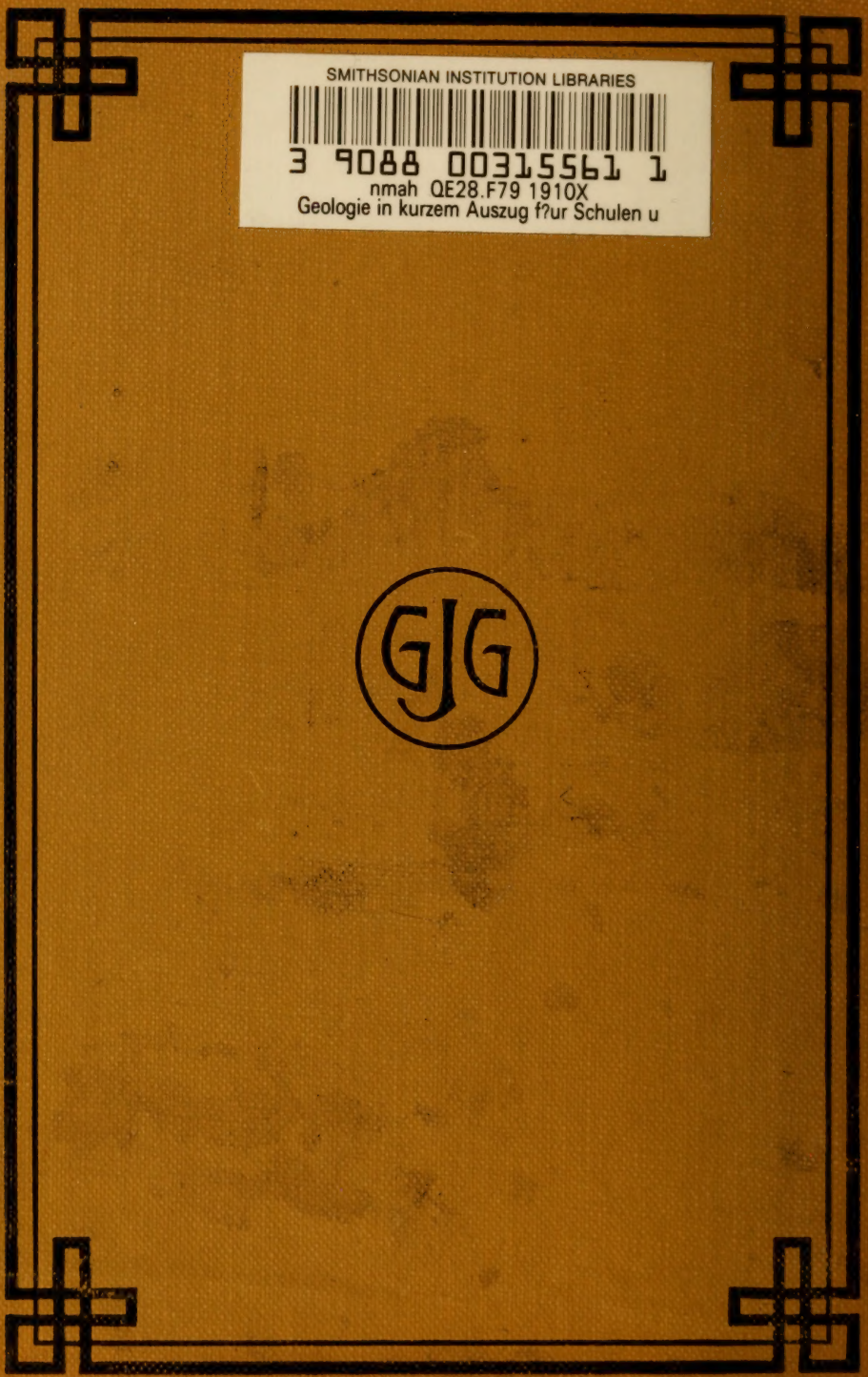

